\title{
PHYSIOLOGICAL RESPONSES OF ADIPOSE TISSUE TO DIFFERENT MODELS OF PHYSICAL ACTIVITY AND INACTIVITY
}

\begin{abstract}
A Dissertation presented to the Faculty of the Graduate School at the University of Missouri
\end{abstract}

In Partial Fulfillment

of the Requirements for the Degree

Doctor of Philosophy

\section{by}

Joseph M. Company

Dr. Frank W. Booth, PhD, Dissertation Supervisor

May 2013 
The undersigned, appointed by the Dean of the Graduate School, have examined the dissertation entitled

PHYSIOLOGICAL RESPONSES OF ADIPOSE TISSUE TO DIFFERENT MODELS OF PHYSICAL ACTIVITY AND INACTIVITY

Presented by Joseph M. Company

A candidate for the degree of Doctor of Philosophy

And hereby certify that in their opinion is worthy of acceptance

Frank W. Booth

M. Harold Laughlin

Douglas K. Bowles

James W. Perfield 


\section{ACKNOWLDEGEMENTS}

In conversation with advisors, visiting professors, public health workers, educators, and parents, it is clear that childhood obesity and inactivity is a major problem. As a passionate health and fitness enthusiast and scientist, I struggle with the balance between "getting the word to the people" and "figuring out the science behind the problem." It seems that in order to be highly successful at either of these, you need to focus on one or the other.

Fortunately, I was able to incorporate my personal passion for health, activity, and exercise into a model of childhood obesity that I am equally passionate about. I believe that the studies presented in the following chapters provide new and further evidence that enforce that it is important to incorporate activity into daily life and remain active throughout life.

This line of research would not have been possible without the help of many people. I would like to name a few people who were integral to my dissertation research. Ryan Toedebusch and Clayton Cruthirds helped tremendously with RNA isolation. Tom Childs is the molecular and technical brains of the Booth lab and many of the assays and analysis couldn't have been performed without his help. Dr. Mike Roberts was my office mate for the final three years of my dissertation research and served as a mentor, consultant, technical assistant, intellectual contributor, and most importantly, a friend.

Finally, I need to acknowledge Dr. Frank Booth, without whom I wouldn't have begun this journey and definitely wouldn't have finished it. 


\section{TABLE OF CONTENTS}

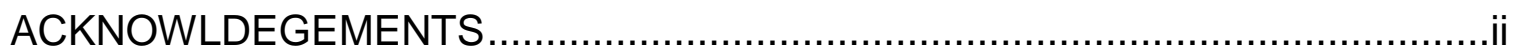

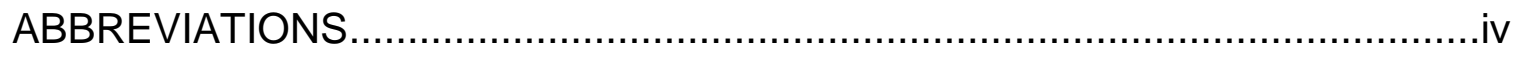

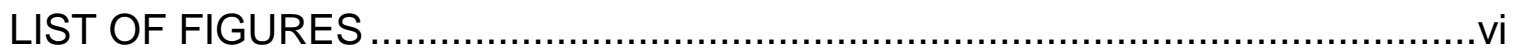

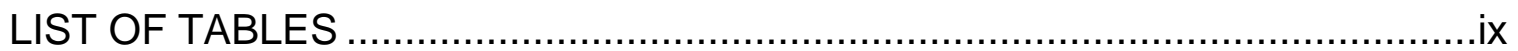

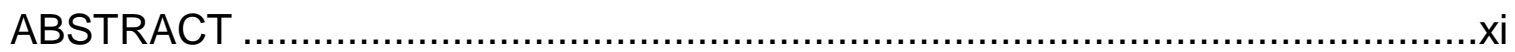

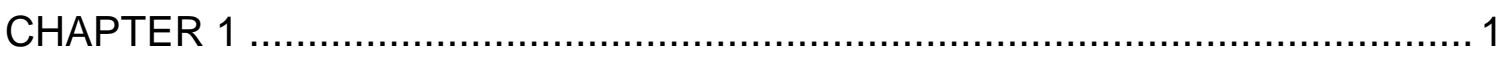

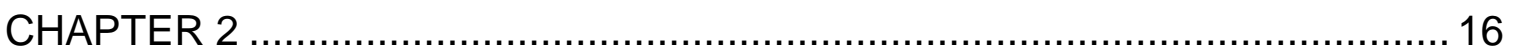

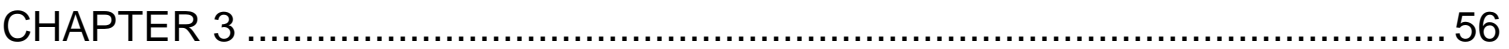

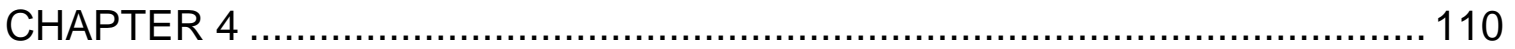

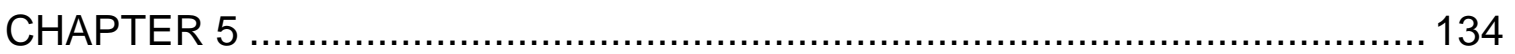

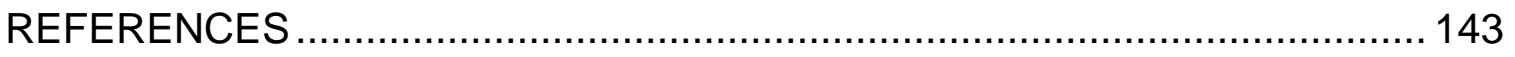

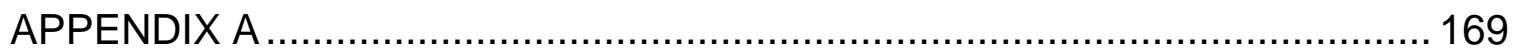

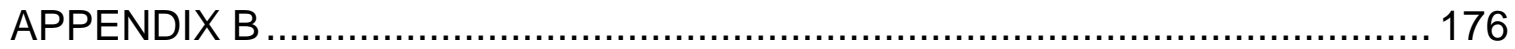

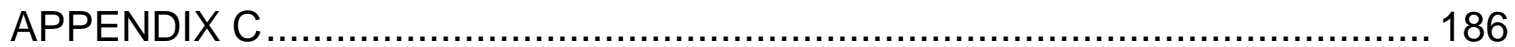

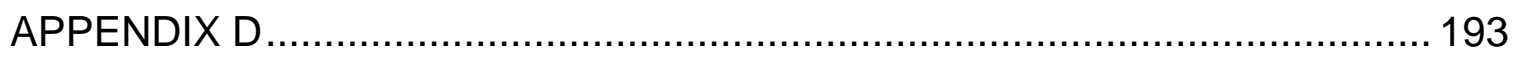

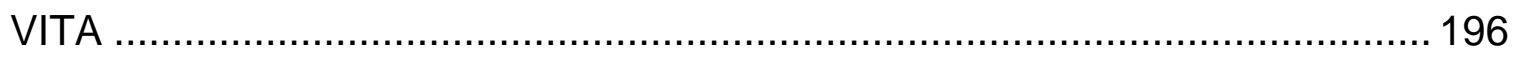




\section{ABBREVIATIONS}

ATGL - adipose triglycerol lipase

AT - adipose tissue

BAT - brown adipose tissue

CDC - Centers for Disease Control and Prevention

DETs - differentially expressed transcripts

DMSO - dimethyl sulfoxide (vehicle for RU486)

DXA - dual-energy $x$-ray absorptiometry

EAT - epididymal adipose tissue

FBN - Fischer 344 x Brown Norway rats

GC - glucocorticoid

GR - glucocorticoid receptor

HSL - hormone sensitive lipase

IPA - Ingenuity Pathway Analysis

LPL - lipoprotein lipase

MSC - mesenchymal stem cells

MGL - monoglycerol lipase

OMAT - omental adipose tissue

PRAT - perirenal adipose tissue

RNA-seq - RNA sequencing

RPKM - reads per kilobase million (final unit of transcript expression)

RT-PCR - reverse transcriptase polymerase chain reaction

RUN - rats with access to a voluntary running wheel

SED - rats without access to a voluntary running wheel 
SQAT - subcutaneous adipose tissue

UCP-1 - uncoupling protein-1

VAT - visceral adipose tissue

WAT - white adipose tissue

$\mathrm{WL}$ - wheel lock

WL+GR BLOCK - wheel lock plus daily administration of RU486 in DMSO

WL+VEHICLE - wheel lock plus daily administration of DMSO

$7 d-W L-7$ days of wheel lock

$11 \beta \mathrm{HSD}-11$ beta hydroxysteroid dehydrogenase

\%BF - percent body fat 


\section{LIST OF FIGURES}

\section{Chapter 1}

Figure 1.1. DXA scans comparing gynoid and android adiposity.................. 4

Figure 1.2. Two mechanisms by which adipose tissue can expand ............. 6

Figure 1.3. Progression from mesenchymal precursor cell to mature adipocyte...8

\section{Chapter 2}

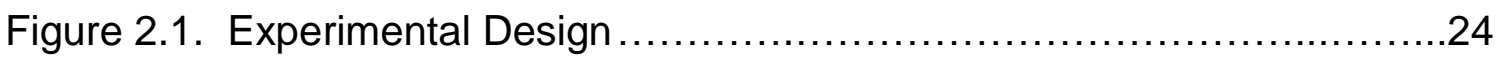

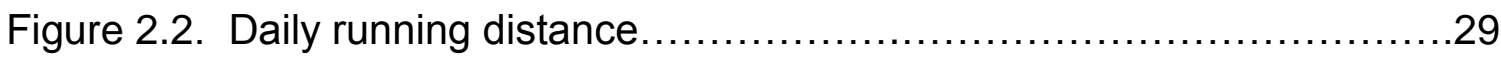

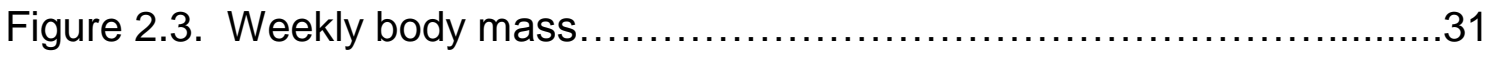

Figure 2.4. Body mass, DXA lean body mass, DXA fat mas, and DXA percent body fat.........................................................

Figure 2.5. Weekly food intake in $70-77$ day old rats...........................34

Figure 2.6. Feed efficiency in $70-77$ day old rats.............................35

Figure 2.7. Adipose tissue depot mass...................................... 37

Figure 2.8. Mean adipocyte diameter and total adipocyte number in depot.......38

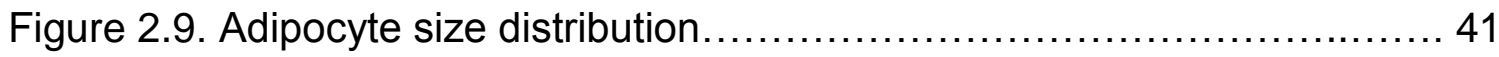

Figure 2.10. mRNA expression of cyclin A1 ............................... 42

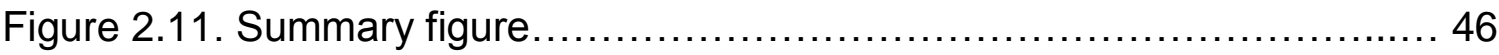




\section{Chapter 3}

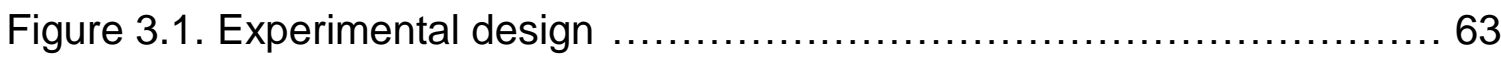

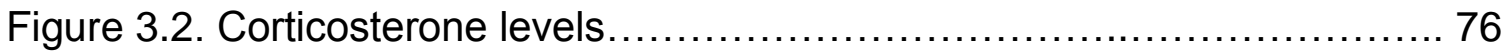

Figure 3.3. Daily food intake over the last 14 days ................................76

Figure 3.4. Perirenal adipocyte size distribution ...............................77

Figure 3.5. Hyothesis-specific RNA-seq results with filtering process to identify transcripts "unique to RUN" and "unique to "BLOCK" ....... 79

Figure 3.6. Top scoring up-and down-regulated networks for transcripts unique

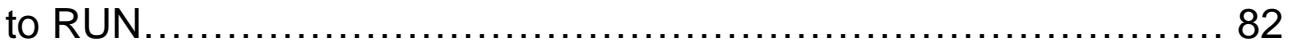

Figure 3.7. Whole transcriptome RNA-seq filtering process and results........ 87

\section{Appendix B}

Figure B1. Dissection procedure for coronary epicardial adipose tissue and myocardial epicardial adipose tissue (mEAT)................. 178

Figure B2. Representative pictures of EX and SED hearts....................180

Figure B3. Correlation of heart weight with total EAT weight for all 13 pigs......180

Figure B4. Intima-media thickness (IMT): grading of lesion of structural atherosclerosis.

Figure B5. Comparison of peri-coronary EAT (cEAT), peri-myocardial EAT (mEAT), and visceral omental adipose tissue (VAT) gene expression to that in subcutaneous adipose tissue (SAT) of sedentary pigs....182

Figure B6. Comparison of peri-coronary EAT (cEAT), peri-myocardial EAT (mEAT), VAT, and SAT gene expression in exercised pigs to that in sedentary pigs 183 


\section{Appendix C}

Figure C1. Experimental design .......................................... 189

Figure C2. Adipocyte size from FHM4 pigs ................................ 190

Figure C3. Lipolysis from FHM4 pigs …................................... 190

Figure C4. 11 $\beta \mathrm{HSD}-1$ enzyme activity in FHM5 pigs .......................... 191

Figure C5. IHC staining for 11ßHSD-1 in FHM5 adipose tissue ................ 191

Figure C6. 11 $\beta$ HSD-1 protein in FHM5 pigs...................................... 192

\section{Appendix D}

Figure D1. Lipolysis assay on plasma of YOUNG, LFD, and HFD rats........ 195 


\section{LIST OF TABLES}

\section{Chapter 2}

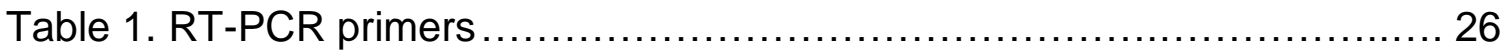

Table 2. RT-PCR results normalized to 70d RUN SQ .......................... 43

Table 3. Summary of outcome measures ..................................... 45

\section{Chapter 3}

Table 3.1. List of 173 hypothesis-specific transcripts.......................... 68

Table 3.2. Body mass, lean body mass, and $\%$ body fat...................... 75

Table 3.3. Adipose tissue depot mass and adipocyte diameter ................. 75

Table 3.4. Transcripts involved in Cell Cycle, Proliferation and Differentiation... 84

Table 3.5. Top-10 transcripts and top networks............................. 88

Table 3.6. Top-10 transcripts expressed in one group but not the other ......... 89

\section{Appendix A}

Table A1. 73 Up-regulated transcripts from RNA-Seq analysis................ 170

Table A2. 44 Down-regulated transcripts from RNA-Seq analysis.............. 172

Table A3. Top up-regulated IPA pathways for RUN vs. WL................... 173

Table A4. Top down-regulated IPA pathways for RUN vs. WL............... 173

Table A5. 14 transcripts that are expressed in RUN but not WL............... 174

Table A6. 32 transcripts that are expressed in WL but not RUN............... 175 


\section{Appendix B}

Table B1. Pig characteristics.............................................. 180

Table B2. Mean maximum and minimum intima-media thickness values...... 181

\section{Appendix C}

Table C1. Pig Characteristics............................................... 189

\section{Appendix D}

Table D1. Anthropometric and adipose characteristics of young, HFD, and LFD rats..194 


\title{
PHYSIOLOGICAL RESPONSES OF ADIPOSE TISSUE TO DIFFERENT MODELS OF PHYSICAL ACTIVITY AND INACTIVITY
}

\author{
Joseph M. Company \\ Frank W. Booth, Ph.D. Dissertation Supervisor
}

\begin{abstract}
Childhood is a critical time of growth, and decisions made during this time lead to positive and negative health consequences, specifically pertaining to adiposity. There is convincing evidence that children who are overweight and obese are at higher risk for adult obesity and risk factors associated with obesity for their whole life course. Puberty is a critical time of adipose tissue expansion, and it is accepted by most that this is the age where the number of adipocytes is set for adulthood. Further it is believed that before puberty, adipose tissue expands by hyperplasia, while after puberty, adipose tissue expands by hypertrophy. The time leading up to puberty represents a critical time. Yet, little is known about the interaction between age, physical activity, and adipose tissue expansion in young, growing animals. Thus, I employed a unique model of childhood obesity to test whether: 1) seven days of decreased physical activity (wheel lock) in 49-56 day old rats and 70-77 day old rats would result in gains in visceral adipose mass seen in age-matched sedentary rats concurrent with an
\end{abstract}


increase in adipocyte size and inflammatory mRNA expression (Chapter 2), and 2) glucocorticoid block would attenuate the visceral adipose depot gains seen with wheel lock in rats at sexual maturity (Chapter 3). Here, I present evidence that the age at which wheel lock occurs influences visceral adipose tissue growth and propose a mechanism for this growth at two ages of pre-pubertal growth (4956 days of age and 70-77 days of age). Specifically, rats that undergo wheel lock for 7 days at 49-56 days of age show no differences in total body mass, mean adipocyte diameter, or mass of omental, epididymal, and perirenal adipose tissue depots compared to rats that remained physically active. However, rats that undergo wheel lock for 7 days at 70-77 days of age have an increased rate of body mass, fat mass, and \% body fat gained and have increased depot mass and total number of adipocytes in epididymal and perirenal adipose tissue, resulting in epididymal and perirenal expansion via hyperplasia. This expansion occurs without an inflammatory component. Further, I present evidence that glucocorticoids play a role in visceral adipose expansion during wheel lock. Blocking glucocorticoid action attenuated rapid growth of adipose tissue mass (catch-up fat) that occurred during wheel lock at the same level that maintaining voluntary wheel running does, but importantly it does not exhibit the same transcript expression as running. Collectively, these findings reinforce the role of the beneficial effects of physical activity. Sustained daily physical activity in young animals maintains lower body fat \%, lowers visceral adiposity and total adipocyte number, which can be translated into a powerful public health message of primary prevention of obesity. 


\section{CHAPTER 1}

\section{Introduction}

Physical inactivity is connected to the obesity crisis facing children and adolescents in the United States (U.S.) [1-3]. There is an increased prevalence of physical inactivity that occurs as children age to adolescents in the U.S. [1, 3] along with an increased portion of children who are obese [4]. Moreover, puberty is a critical time of adipose tissue expansion [5] where the number of adulthood adipocytes is generally set [6]. Fewer whole-body adipocytes and less fat mass accumulation during childhood is correlated with a decrease risk of obesity and all of the accompanying obesity-related problems later in life [7]. It is clear that decisions made early in life have major health effects later in life. Taken together, we see an earlier onset of obesity and an increase in physical inactivity in children occurring at a critical time of adipose tissue growth. Given these facts, animal models that allow the study of adipose tissue plasticity within the abdominal cavity in an inactivity context are valuable to gain an understanding of the potential negative consequences of early childhood inactivity and on fat gain. Preforming studies that obtain visceral fat biopsies from healthy and unhealthy children is unethical, so animal models are required to obtain medical information for the health of children. Our laboratory has a unique model that is effective at investigating this, and was the model of choice for the two studies presented in this dissertation [8]. The overlying theme of this dissertation is how decreased 
physical activity affects adipose tissue in a rodent model of childhood obesity.Rats are a good model for children in regards to studying adipose tissue for the following reasons: 1) rats gain body mass (first 11 weeks of life) in parallel with gains for children (first 20 years of life) [http://www.cdc.gov/growthcharts/clinical_charts.htm and [9]], 2) the number of adipocytes in rats dramatically increases in pre-pubescent years and then plateaus thereafter just as is observed in humans $[6,10]$, and 3 ) in rats, we are able to have access to all tissue for molecular investigation [8]. For these reasons, young, Wistar rats were selected for the two studies presented in this dissertation.

The Booth Laboratory Wheel Lock (WL) Model is a unique, effective way of studying the effects of a sudden decrease in physical activity in the context of children and adolescents [8], and is described in detail in Chapter 2 and Chapter 3. An example of this paradigm is children ending a summer of play to sit in school is a human example of decreased activity. It is important to note that the WL model is very novel, and few studies have been performed using a WL model, but that does not mean WL is irrelevant. Rather, I would argue that WL is a highly human-relevant, yet still underappreciated, model of human behavior, which provides another model to study decreased activity at a molecular level.

\section{Adipose tissue is an endocrine organ}

Adipose tissue was once thought of as a passive tissue functioning solely to store excess triglycerides. This view is now universally rejected due to a 
plethora of evidence showing the diverse metabolic activity of adipose [11]. Adipose tissue can generally be classified as either white adipose tissue (WAT) or brown adipose tissue (BAT). BAT is characterized by expression of uncoupling protein-1 (UCP-1) and is considered a depot more metabolically active and is specialized for heat production [12]. Recent literature suggests a cross-over type of fat called BRITE (BRown-whITE) or beige fat, which is characterized by a white adipocyte that can express UCP-1 $[13,14]$. WAT makes up the majority of adipose tissue in humans and rodents, and is the predominant type of adipose tissue in mammals $[11,15]$ and is the focus of this dissertation.

Adipocytes make up the majority of adipose tissue volume, but there are many other multipotent cell types in adipose tissue, referred to as the stromal cells. Cells in the stromal fraction have the capacity to differentiate into adipocytes, chondrocytes, and osteoblasts, as well as secrete cytokines and growth factors (referred to as adipokines) with autocrine, paracrine, and endocrine functions [16]. Due to the large size of whole-body adipose tissue and the extensive vascularization, adipose tissue has the ability to release vast quantities of adipokines into the systemic circulation and significantly affect body functions [17]. It has been suggested that adipose tissue is the source of many of the inflammatory markers that are responsible for the increase in circulating pro-inflammatory cytokines [18]. With increased obesity and an increase in adipocyte size, there is an adipose tissue phenotype shift from an antiinflammatory immune environment to a pro-inflammatory immune environment 
that is concurrent with the development of metabolic complications like insulin resistance and type 2 diabetes [19]. Specifically, there is a chronic state of lowgrade inflammation, [20] an increase in circulating pro-inflammatory cytokines including TNF $\alpha$, IL-6, and monocyte chemotactic protein-1 (MCP-1) [21], and a decrease in anti-inflammatory cytokines like IL-10.

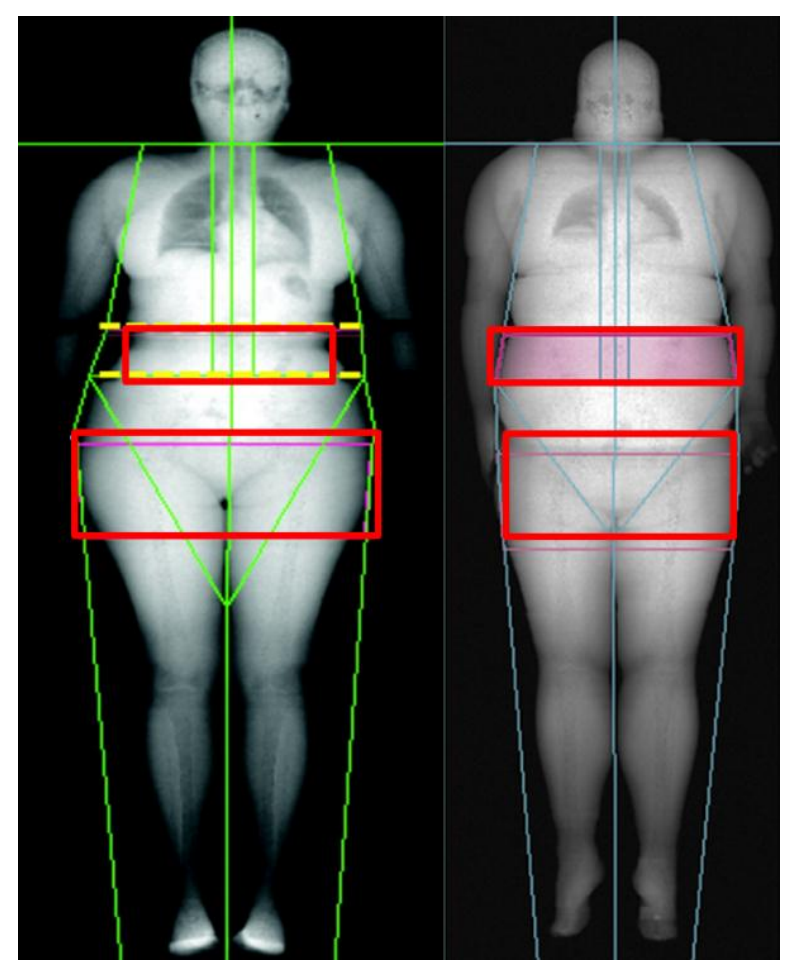

FIGURE 1: DXA scans comparing gynoid and android adiposity. Gynoid or hip and thigh adiposity at left versus android or central adiposity at right (images from: [[22] and gehealthcare.com]. Waist and hip are outlined with a red box. Andriod adiposity, especially when the adipose tissue is located in the viscera, poses the highest risk for health problems. Note in gyonid adiposity (left) the smaller waist and larger hip, while in android adiposity (right) the larger waist and smaller hip.

Location of adipose tissue within the body is heterogeneous with different depots exerting a variety of functions [23]. While subcutaneous AT (SAT), often distributed in the hip and thigh area is considered "protective fat depot" [24, 25], 
central adiposity, especially visceral adipose tissue (VAT), is implicated in metabolic and chronic inflammatory disorders [23] and is considered to be potentially pathogenic and predisposes people to higher risk of disease than other depots [26-34]. Therefore central, abdominal obesity, referred to as android adiposity, is considered a risk factor for metabolic syndrome, while peripheral, hip and thigh obesity, which is referred to as gynoid obesity, is not (Figure 1).

It has previously been reported that there are metabolic differences between subcutaneous and VAT and even between different VAT depots [35]. Despite the differences, results from the Framingham Heart Study, which investigated 3001 healthy adults, found that SAT and VAT were positively associated with hypertension, triglycerides, HDL cholesterol, and insulin resistance [24], suggesting that increased adiposity, no matter the location, puts people at higher risk for disease. Still the authors of the Framingham Heart Study found that VAT is more highly correlated with metabolic syndrome than SAT. Two main reasons that VAT is considered to be "unhealthy" is 1) the close proximity to the liver and 2) the inherent damaging molecular characteristics [30]. A proposed mechanism by which VAT exerts harmful metabolic effects is that there is an enhanced efflux of inflammatory cytokines and free fatty acids through the portal vein to the liver [36]. Omental (OMAT) is VAT most commonly cited in human studies, while epididymal (EAT) and perirenal (PRAT) depots are commonly investigated in rodent studies. As will be presented later, OMAT responds differently to treatments than EAT and PRAT. VAT, specifically OMAT, 
EAT, and PRAT adipose tissue depots were investigated in this dissertation due to their highly plastic nature as well as their positive correlation to metabolic risk.

\section{Adipose tissue expands by hypertrophy or hyperplasia}

AT is highly plastic and can rapidly change based on animals physiological and pathophysiological status [37-40]. Adipose tissue can expand by either hypertrophy (increase in the size of an adipocyte) or hyperplasia (increase in the number of adipocytes) (Figure 2).

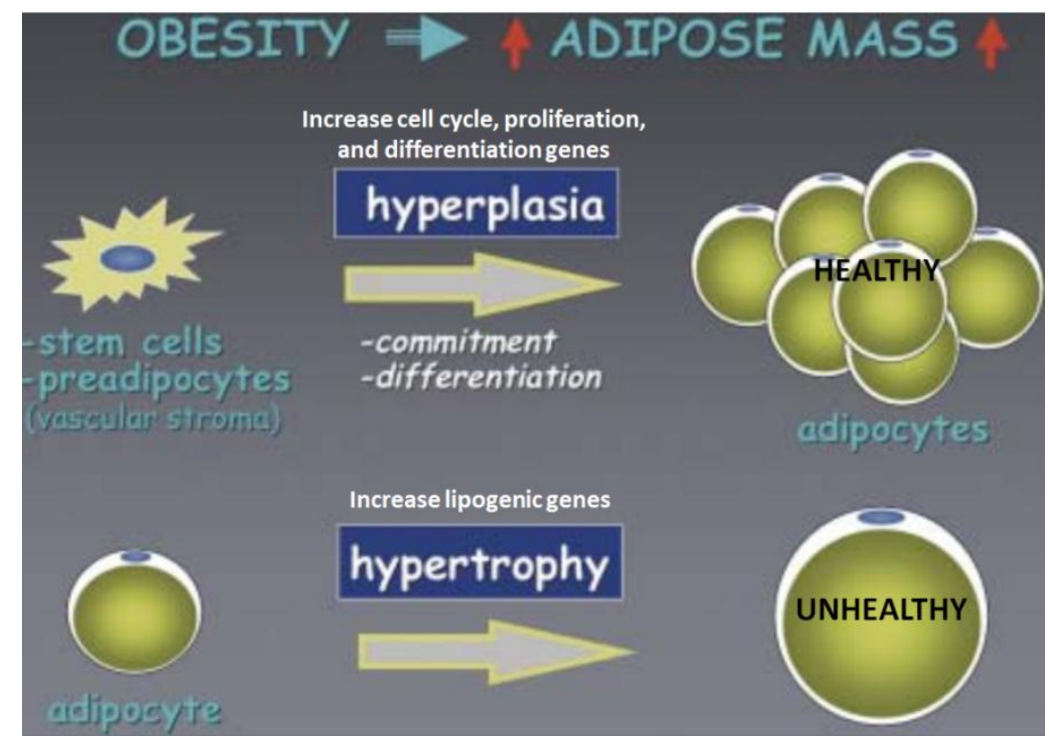

FIGURE 2. Two mechanisms by which adipose tissue can expand.

Hyperplasic expansion originates from preadipocyte differentiation and expands adipose tissue by adding more adipocytes. Hypertrophic expansion involves in increased lipid storage in existing adipocytes and expands adipose tissue by making already-existing adipocytes larger. Figure was modified from [41]

Hyperplasia results from stem cell recruitment and differentiation into new, small adipocytes and a consequent increase in total adipocyte number in a given depot [42], and is generally a "healthy" adipose tissue adaptation due to 
maintenance of smaller adipocytes [43]. The stromal fraction of adipose tissue contains pluripotent mesenchymal stem cells (MSCs) that can undergo commitment and differentiation into adipocytes, myocytes, chondrocytes, and osteocytes [44-47]. MSCs are recruited to the adipocyte lineage by excessive energy intake in vivo [48], which induce MSCs towards preadipocyte hyperplasia and differentiation into the adipocyte phenotype and enlargement of mature adipocoytes [49]. Figure 3 illustrates many of the known markers of the process from MSC to mature adipocyte.

As mentioned previously, childhood, specifically puberty, is a critical time of adipose tissue expansion [5], and it is currently accepted by the majority of scientists who study adipose tissue, that the number of adulthood adipocytes is generally set during this time $[6,50,51]$. Smaller adipocytes are considered to be healthier [52], and as a result, adipose tissue expansion via hyperplasia results in a healthier phenotype than adipose tissue expansion via hypertrophy [53] even in the presence of obesity [54]. This evidence suggests that one of the keys to avoiding these metabolic complications is to maintain healthy adipose tissue (i.e. small adipocytes and an anti-inflammatory environment.

Hypertrophy involves increased lipid storage in pre-existing adipocytes and a resultant need for adipose tissue remodeling to accommodate the larger adipocytes, which often is accompanied by an inflammatory process [55]. Therefore, it is believed that adipose tissue expansion by hypertrophy, especially VAT expansion via hypertrophy, leads to an unhealthy phenotype that is concurrent with the development of metabolic complications and is a risk factor 
for type 2 diabetes and cardiovascular disease $[19,33,40,56,57]$. Much of the work on adipose expansion has been done with high fat diets, while very few studies investigate adipose expansion with decreased activity. Therefore, one of the major questions addressed in Chapter 2 is whether visceral adipose tissue expansion during WL occurs via hypertrophy or hyperplasia, and is this accompanied with an inflammatory component.

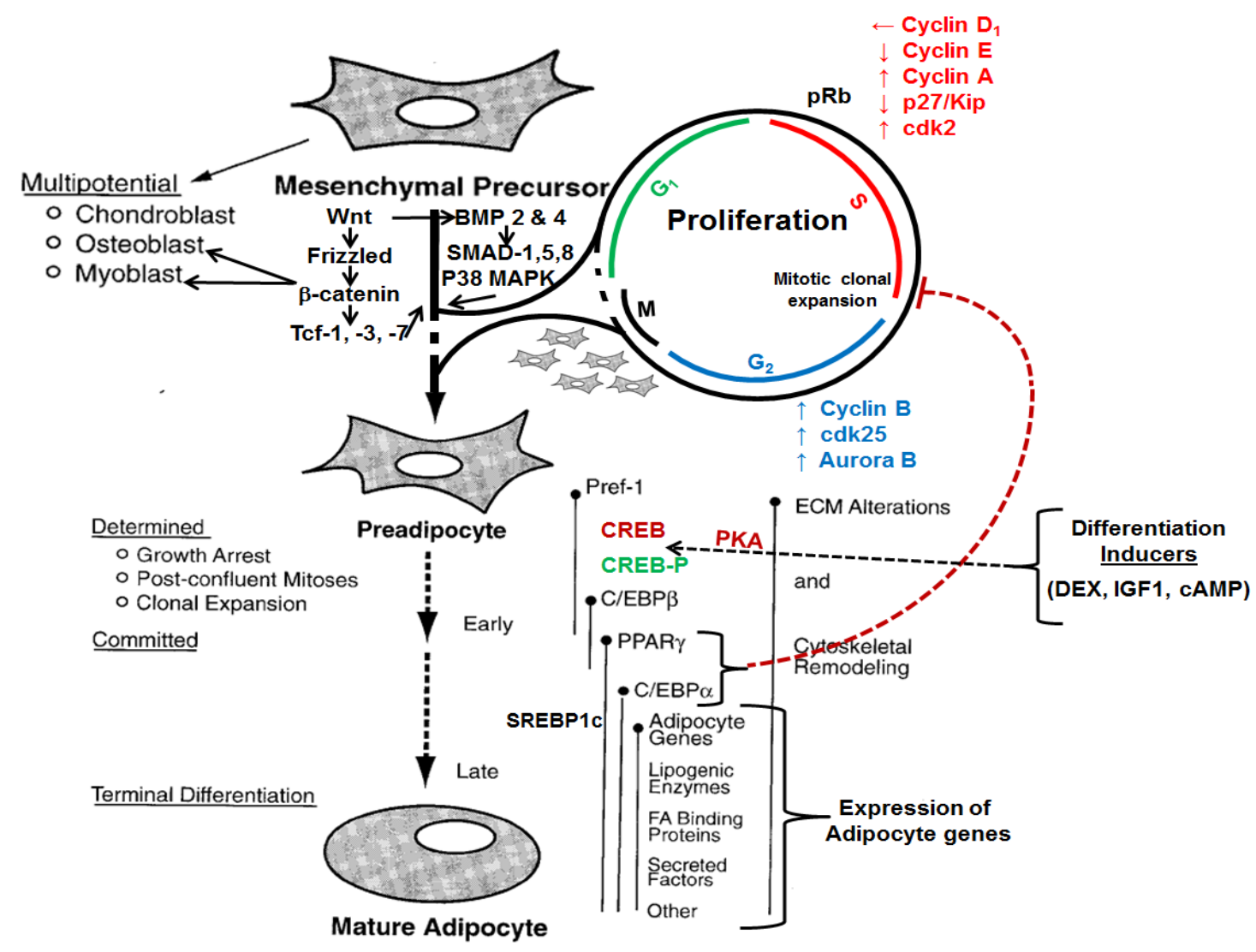

Figure 3. Progression from mesenchymal precursor to mature adipocyte. Abbreviations: Wnt, wingless-type MMTB integration site family; Tcf, transcription factor; BMP, bone morphogenetic protein; MAPK, mitogen-activated protein kinase; cdk, cyclin-dependent kinase; p27/Kip, cyclin-dependent kinase inhibitor; $\mathrm{pRb}$, retinoblastoma protein; CREB, cyclin AMP regulatory elementbindingprotein; PKA, protein kinase A; PPAR $\gamma$, peroxisome proliferator-activated receptor-gamma; C/EBP, CCAAT/enhancer-binding protein; SREBP1c, sterol regulatory element binding protein 1c; DEX, dexamethasone; IGF1, insulin-like growth factor 1; cAMP, cyclic AMP; $G_{1}, S, G_{2}$, and $M, G_{1-}, S-, G_{2^{-}}$, and M-phases of the cell cycle. Figure 3 was adapted from $[42,58]$. 


\section{Catch-up fat occurs during calorie restriction-then-re-feeding and also during a model of physical activity-then-inactivity}

Catch-up fat is the growth of AT observed once a cause of normal growth is removed [59] and is often accompanied by an increase in abdominal obesity $[40,56,57]$. In addition, the AT phenotype that develops during catch-up growth is characterized by increased secretion of pro-inflammatory adipokines [59]. This is commonly investigated by under- then over-nutrition [59], but this also occurs in activity/inactivity models as both rodent models (wheel lock) and human models (decreased steps per day) exhibit 'catch-up fat' [60-62]. Another human correlate is the Dutch famine of 1944-1945, where exposure to famine during gestation and childhood was associated with impaired glucose tolerance, obesity, coronary heart disease, and hypertension in adulthood [63]. The catch-up fat concept is applicable to children's environment, where they drastically decrease their daily activity after a summer vacation and sit in a classroom. The majority of the literature on catch-up fat utilizes a calorie-restriction then re-feeding strategy. Therefore, a major difference between our WL model and catch-up fat literature is mechanistic differences. The WL model induces catch-up fat via increased voluntary physical activity followed by decreased caloric expenditure, while nutrition-based catch-up models decrease calories, then increase caloric intake.

The reasons that this so-called 'thrifty catch-up-fat phenotype' exists and why catch-up growth produces harmful later health effects is not known [56, 64]. However, the accelerated visceral AT expansion and switch to an 'unhealthy,' 
pro-inflammatory phenotype documented during catch-up growth, is a likely contributor [65]. We have observed catch-up fat in studies performed in the Booth lab during periods of WL [60, 61], much like catch-up fat discussed in nutrition literature [59]. Both Chapter 2 and Chapter 3 address this question of catch-up fat during WL, but in two different contexts. In Chapter 2, I investigate catch-up fat in two ages of young rats, while in Chapter $3, I$ investigate the effects of glucocorticoids on catch up fat.

\section{Physical activity maintains lower adiposity as well as a healthier adipose tissue phenotype}

Chronic physical activity is an important non-pharmacological means to reduce adiposity and disease risk. Among a long list of benefits that physical activity has on physiology, protection against weight gain, decreased body fat, and a healthier adipose tissue phenotype are among them [66-69]. One way exercise does this is by maintaining lower levels of visceral adipose mass [70]. Interestingly, exercise training can result in AT mass reduction, specifically VAT, independent of body mass index and/or total body weight $[32,71]$. Chronic physical activity, like endurance training, has many other effects, one of which is that insulin-sensitive tissues like adipose tissue, skeletal muscle, and liver become more sensitive to glucocorticoids (GCs) [72]. In adipose tissue, a heightened sensitivity to GCs is beneficial in that there is increased lipolysis [73], which provides a valuable energy source for physically active animals. However, 
this increased sensitivity to GCs and adipose tissue lipolysis could have a detrimental effect for sedentary animals through increased non-esterified fatty acids and adipose tissue redistribution towards the viscera [74, 75]. An extreme example is Cushings Syndrome, which occurs due to inappropriately high levels of cortisol in humans. In this context, I choose to investigate the role of GCs in the catch-up fat paradigm. I wondered if the potential heightened sensitivity to GCs combined with a sudden decrease in physical activity contributes to the increased adiposity seen in our lab's prior WL studies.

\section{Glucocorticoids and adiposity}

Glucocorticoids (cortisol in humans and corticosterone in rodents) are of interest because of they are known to affect adipose tissue metabolism. While the role of GCs in liver and skeletal muscle is relatively clearly defined, the role of GCs in adipose tissue is controversial. GCs are most commonly believed to be lipolytic [76], but GCs promote preadipocyte differentiation and adipogenesis [77]. GCs target, the glucocorticoid receptor (GR), is more highly expressed in visceral fat than other fat depots [78], and increased exposure to GCs in insulinsensitive tissues is associated with lipid accumulation and metabolic complications [79-83]. Interestingly, GCs affect different fat depots differently; they increase lipolysis in peripheral fat depots, like SAT, but they promote preadipocyte differentiation and adipocyte hypertrophy in VAT [84], so there is something unique to VAT regarding GC action. GC treatment in children for 
conditions such as asthma and rheumatic diseases, inhibit weight-loss efforts and aggravate weight gain [85-87], and rodents with reduced GC action through a GC receptor block show attenuated fat increases and even induce fat loss [88]. Because of this strong connection between GCs and adipose tissue and their potential to promote adiposity, I investigated the role of GCs in the inactivityinduced catch-up fat (Chapter 3).

\section{Prior wheel lock studies and genesis of hypotheses}

Two WL studies previously published in the Booth laboratory that specifically reported on adipose tissue catch-up during WL had contradictory results. Both studies used the same age (rats were 28 days of age at the onset of voluntary wheel running) Fischer-Brown Norway F1-generation rats with ad libitum access to standard rat chow. In the first study, in 2005 Kump \& Booth [61] reported that after 3 weeks of voluntary running and 2 days WL, EAT depot mass increased, mean adipocyte size increased, but total adipocyte number did not. Kump concluded that EAT expanded by hypertrophy during the 2 days of WL, and that the increase was due to a prolonged overshoot in triacylglycerol synthesis. Then, in the second study in 2007, Laye et al. [60] reported that after 6 weeks of running and 2 days of WL, EAT depot mass did not increase, mean adipocyte size did not increase, and adipocyte number did not increase. In contrast to a 2-day WL, Laye et al. reported that 7 days of WL had the opposite effect. EAT depot mass and total adipocyte number increased, while mean 
adipocyte size did not increase. In addition, Laye showed that this result is not due to overeating, since a pair-fed group of rats showed the same effect. Laye concluded that EAT depot expanded by hyperplasia during the 7 days of WL. Both studies confirm that adipose tissue expands with WL. Kump's data showed hypertrophy, while Laye's data showed hyperplasia. There was one outstanding difference in experimental design; Kump performed measurements on 49-51 day old rats with 2 day WL, while Laye performed measurements on 70-77 day old rats with 7 day WL.

These seemingly contradictory studies inspired my dissertation research. Given the important role adipose tissue plays in health, the dynamic nature of adipose tissue, and the unknown mechanism by which adipose tissue expands with WL, my questions were: 1) Does the way adipose tissue expands depend on the age of WL? (if we WL at a younger, pre-pubertal age, do we see hypertrophy and an older, pre-pubertal age do we see hyperplasia?) 2) How does adipose tissue expand with one week of WL? (hypertrophy or hyperplasia?) 3) Is this expansion healthy or unhealthy? (is there an inflammatory component that accompanies fat gains during WL?) 4) Do glucocorticoids play a role in this adipose tissue expansion? (if we block GC action, could we attenuate the fat mass gains?). I used two different ages of rats (49-56 days of age and 70-77 days of age) to represent the two previous studies performed in our lab as well as represent two ages of pre-pubertal growth. 49-56 days of age is prior to-sexual maturity, while $70-77$ days of age is near or atsexual maturity [89]. 


\section{Overall Hypothesis and specific aims for the two studies presented}

My overall hypothesis was that seven days of wheel lock in rats that are 49-56 days old and rats that are 70-77 days old would result in gains in visceral adipose mass seen in age-matched sedentary rats concurrent with an increase in adipocyte size and inflammatory mRNA expression. Further, glucocorticoid block would attenuate the visceral adipose depot gains seen in WL in 70-77 day old rats.

Specific hypothesis in Chapter 2: I tested the hypothesis that 7 days of wheel lock would: a) increase the rate of percent body fat growth and adipose depot masses to levels seen in age-matched SED rats in both ages of rats; b) result in a visceral adipose depot expansion via hypertrophy in rats 49-56 days of age and via hyperplasia in rats 70-77 days of age, and c) increase inflammatory mRNAs in adipose tissue before other selected categories of mRNAs in rats 49-56 days of age but not 70-77 days of age. The aim of this study was to 1) address how adipose tissue expands with $\mathrm{WL}$ and determine whether an increase in inflammatory mRNA expression is present and 2) determine whether timing of WL (49-56 days of age or 70-77 days of age) affects outcomes of adipocyte size and number, and of whether inflammatory mRNAs profile would differ between these two groups.

Specific hypothesis in Chapter 3: I tested the hypothesis that when compared to WL rats, rats that had "access to voluntary running wheels" and rats that "underwent WL plus a glucocorticoid receptor block" would show similar changes 
as "WL rats" in PRAT transcript expression involved in cell cycle, proliferation, and differentiation. This hypothesis was generated from the finding in Chapter 2 where we found that cyclin A1, a marker of proliferation and total adipocyte number was increased in PRAT and EAT depots in W $L$ rats. 


\section{CHAPTER 2}

\section{STUDY 1:}

SUDDEN DECREASE IN PHYSICAL ACTIVITY EVOKES ADIPOCYTE HYPERPLASIA IN 70-77 DAY OLD RATS, BUT NOT 49-56 DAY OLD RATS 


\section{ABSTRACT}

The cessation of physical activity in rodents and humans initiates obesogenic mechanisms. The overall purpose of the current study was to determine how the cessation of daily physical activity in rats at 49-56 days of age and at 70-77 days of age via wheel lock affects adipose tissue characteristics. Male Wistar rats began voluntary running at 28 days old and were either sacrificed at 49-56 days old or at 70-77 days old. Two cohorts of rats always had wheel access (RUN), a second two cohorts of rats had wheel access restricted during the last seven days (7d-WL), and a third two cohorts of rats did not have access to a voluntary running wheel after the first 6 days of running (SED). We observed more robust changes with WL in the 70-77 day old rats. Compared to RUN rats, $7 d-W L$ rats exhibited greater rates of gain in fat mass and \% body fat, increased adipocyte number, higher percentage of small adipocytes, and greater cyclin A1 mRNA in epididymal and perirenal adipose tissue. In contrast, 49-56 day old rats had no change in most of the same characteristics. There was no increase in inflammatory mRNA expression in either cohort with WL. There was a disconnect between decreased food intake and increased adiposity, suggesting that reduced caloric intake does not reduce body fat when physical activity is reduced simultaneously. These findings suggest that adipose tissue in 70-77 day old rats is more greatly affected by WL than 49-56 day old rats and responds by expansion via hyperplasia. 


\section{INTRODUCTION}

Increased childhood physical inactivity is associated with the rise in childhood obesity. Specifically, an increased prevalence of physical inactivity occurs as children age to adolescents in the U.S. [1, 3]. While over $70 \%$ of girls and over $95 \%$ of boys 6 - to 8 -yrs old meet the recommend daily activity guidelines of 60 minutes of moderate- to vigorous activity daily, less than $15 \%$ of girls and less than $43 \%$ of boys 12 - to 14 -yrs old meet the same requirements. In addition, the U.S. prevalence of obesity was $12.1 \%$ among children aged 2 through 5 years, $18.0 \%$ among children aged $6-11$ years old, and $18.4 \%$ among adolescents aged 12 through 19 years old [4]. Thus, obesity prevalence increases $\sim 50 \%$ when advancing from the $2-5$ year old age group to the $6-11$ year old age group, but does not further increase in the 12-19 year old age group. Taken together, these statistics highlight a growing crisis in public health as children become less active, which coincides with fat gain during decisive periods of growth and development that include various ages of pre-pubertal growth.

Adipose tissue is now well-recognized as an endocrine organ capable of demonstrating an inflammatory/immune component [90, 91]. Further, the function of adipose tissue depends on its localization [23] with visceral fat more closely associated with an increased risk of developing a metabolic syndrome [92, 93]. Adipose tissue is highly plastic and responds rapidly to environmental changes and can expand by either hypertrophy (increase in the size of an adipocyte) or hyperplasia (increase in the number of adipocytes). It is believed that adipose tissue expansion by hypertrophy, especially visceral adipose tissue 
(VAT) expansion, leads to an unhealthy, pro-inflammatory phenotype that is concurrent with the development of metabolic complications and is a risk factor for type 2 diabetes and cardiovascular disease [19, 33, 40, 56, 57]. Conversely, adipose tissue expansion via hyperplasia retains a healthy, anti-inflammatory phenotype that is associated with positive metabolic consequences including maintained insulin sensitivity, and favorable blood lipids [53] even in the presence of obesity [54]. Dulloo [40] has described large decreases in body weight followed by weight recovery occur during infancy, childhood, or adulthood. In most cases, body fat, specifically visceral fat, is recovered via hypertrophy at a disproportionately faster rate than lean tissue (termed "catch-up fat"). These concepts regarding fat gain are important to the childhood obesity conversation because puberty is a critical time of adipose tissue expansion [5] where the number of adulthood adipocytes is generally set [6]. Taken together, it is clear that environmental events early in life influence adipocyte number, adipose tissue distribution, and adipose tissue plasticity later in life [94]. Still, insufficient information is known about the acute mechanisms behind adipose tissue expansion at various ages of pre-pubertal growth.

Animal models that allow study of adipose tissue plasticity within the abdominal cavity in an inactivity context are invaluable in gaining an understanding into potential negative consequences of early childhood inactivity and fat gain. Our laboratory has successfully employed a "rodent wheel lock model" (abbreviated "WL") where we allow pre-pubertal rats access to a voluntary running wheel for a period of time (4-6 wks) and then "lock" the wheel 
for one week to study the effects of transitioning from high levels of physical activity to low levels of physical activity on adipose tissue expansion $[8,60,61$, 95]. In essence, the environmental factor of physical activity is "knocked down", analogous to knock out of a gene to study gene-environment interaction. The notion is somewhat similar to the concept of "catch-up" fat [59], whereby refeeding occurs after caloric restriction. Distinctively though, the genesis of enhanced adipose tissue growth differs between the WL and "catch-up" fat models. We speculate that subtle mechanistic differences between the two processes. The WL model increases voluntary physical activity then decreases physical activity, implying decreased caloric expenditure, which suggests that a physical activity-induced metabolic change influences adipose metabolism. The "classical" catch-up fat model that decreases calories, then increases positive caloric intake suggests a net caloric balance influence on adipose metabolism. Importantly, both models induce the trajectory in adipose tissue mass to turn upward.

Previous work in our laboratory showed that WL resulted in the following adipose tissue changes: 1 ) in 49-day old male rats, 2 days of WL induced an increase in epididymal fat mass via hypertrophy [61]; while 2) in 70-day old male rats, one week of WL resulted in the epididymal adipose tissue depot expansion via hyperplasia [60]. As these differences remain unresolved, we hypothesized that this incongruity could be related to the onset of WL occurring at 49 days of age versus 70 days of age. Therefore, to further understand the dynamic adaptation of fat during WL, the overall purpose of this experiment was to 
determine what role pre-pubertal age (49-56 days of age versus $70-77$ days of age) plays in affecting catch-up growth of adipose tissue mass and adipocyte size in three visceral depots when physically active male rats undergo one week of WL (7d-WL). Two specific purposes were to determine whether WL in 49-56 day of rats and/or 70-77 days of age differentially altered the following: 1) adipose tissue characteristics (mass and/or adipocyte size and adipocyte number); and 2) the mRNA expression of pro-inflammatory, hypoxic, adipokine, and adipogenic genes. Of note, age-matched sedentary controls (SED) and rats that had continual running wheel access (RUN) were also assayed in order to stratify WL animals between these two control groups. We hypothesized that 7 days of WL would: 1) increase the rate of percent body fat growth and adipose depot masses to levels seen in age-matched SED rats in both ages of rats; 2) result in a visceral adipose depot expansion via hypertrophy in rats 49-56 days of age and via hyperplasia in rats 70-77 days of age; and 3) increase inflammatory mRNAs in adipose tissue before other selected categories of mRNAs in rats 4956 days of age but not 70-77 days of age.

\section{METHODS}

\section{Animals and Experimental design}

The University of Missouri animal Care and Use Committee approved experimental protocols. Two similar protocols were employed at two distinct age groups: Protocol 1, "49-56 day old rats" and Protocol 2, "70-77 day old rats" The rationale for this is that we have previously employed the WL model on two ages of rats with differing results on hyperplasia and hypertrophy of adipocytes [60, 
61]. Therefore, we performed WL on two ages, 49-56 days old and aged 70-77 days old to: 1) match previous ages performed in our laboratory [60, 61, 95] and 2) determine if there was an age-related difference in characteristics of adipocyte enlargement in response to WL.

Protocol 1. 34 male, Wistar rats were randomly selected from a population of Wistar rats bred in our laboratory (Fig. 2.1A). The rats were weaned at 21 days of age and housed as pups in a single cage. At 28 days of age, rats were placed into individually housed cages with a voluntary running wheel equipped with a Sigma Sport BC 800 bicycle computer (Cherry Creek Cyclery, Foster Falls, VA) in order to select the highest distance runners in an experiment whose primary outcome was selective breeding of high voluntary distances [96]. After 6 days, 12 rats were randomly selected to be sedentary for the remainder of the experiment for 21 or 28 days (49d SED and 56d SED), were removed from the cages with voluntary running wheels, and were individually housed in standard rat cages without voluntary running wheels respectively (note that the first number in the group name refers to age of rats). The remaining 22 rats were randomly assigned to running groups (49d RUN, 56d RUN and 56d 7d-WL) and remained in cages with voluntary running wheels (note that $7 \mathrm{~d}-\mathrm{WL}$ in the group name refers to 7 days of wheels being locked for the final 7 days of the treatment period). At the end of week 3,8 rats were randomly selected to undergo WL, whereby a stainless steel rod was placed through the running wheel, thus prohibiting the running wheel from turning $(56 d 7 d-W L)$. All rats were housed in the same temperature-controlled animal room $\left(21^{\circ} \mathrm{C}\right)$ and maintained on a $12-\mathrm{h}$ 
light $(07.00$ h- 19.00 h), 12-h (19.00 h-07.00 h) dark cycle. All rats had ad libitum access to standard rodent chow (Formulab 5008, Purina Mills, St Louis, MO, USA) and water. Running activity, as determined by distance and time ran during the previous $24 \mathrm{~h}$, was recorded daily. Animal cages were changed every 7 days at which time body weight and weekly food intake was recorded. This protocol had been used previously in our lab [61].

Protocol 2. 40 male, Wistar rats were randomly selected from a population of Wistar rats bred in our laboratory (Fig. 2.1B). The design is the same as Protocol 1 except for duration of wheel running being 3 wks longer, the day of WL (day 70), and day of sacrifice (day 77) (see Fig. 2.1B for details). In short, rats in Protocol 2 began treatment at the same age as rats in Protocol 1, but were 3 wks older at the time of WL and sacrifice and thus were exposed to voluntary running wheels (70d RUN, $77 d \mathrm{RUN}$, and $77 \mathrm{~d} 7 \mathrm{~d}-\mathrm{WL}$ ) or no voluntary running wheels (70d SED and 77d SED) 3 wks longer than the rats in Protocol 1. During the final week, food intake for $77 d \mathrm{~d} d-W L$ was measured daily. This protocol had been used previously in our lab $[60,61]$.

\section{Animal sacrifice}

Immediately after $\mathrm{CO}_{2}$ asphyxiation between $1000-1200 \mathrm{hrs}$, rats were weighed and DXA scanned as mentioned previously. Four fat pads were collected in Protocol 1 and 2: omental adipose tissue (OMAT), epididymal adipose tissue (EAT), perirenal adipose tissue (PRAT), and subcutaneous adipose tissue (SAT). The entire OMAT, EAT, and PRAT depots were removed 


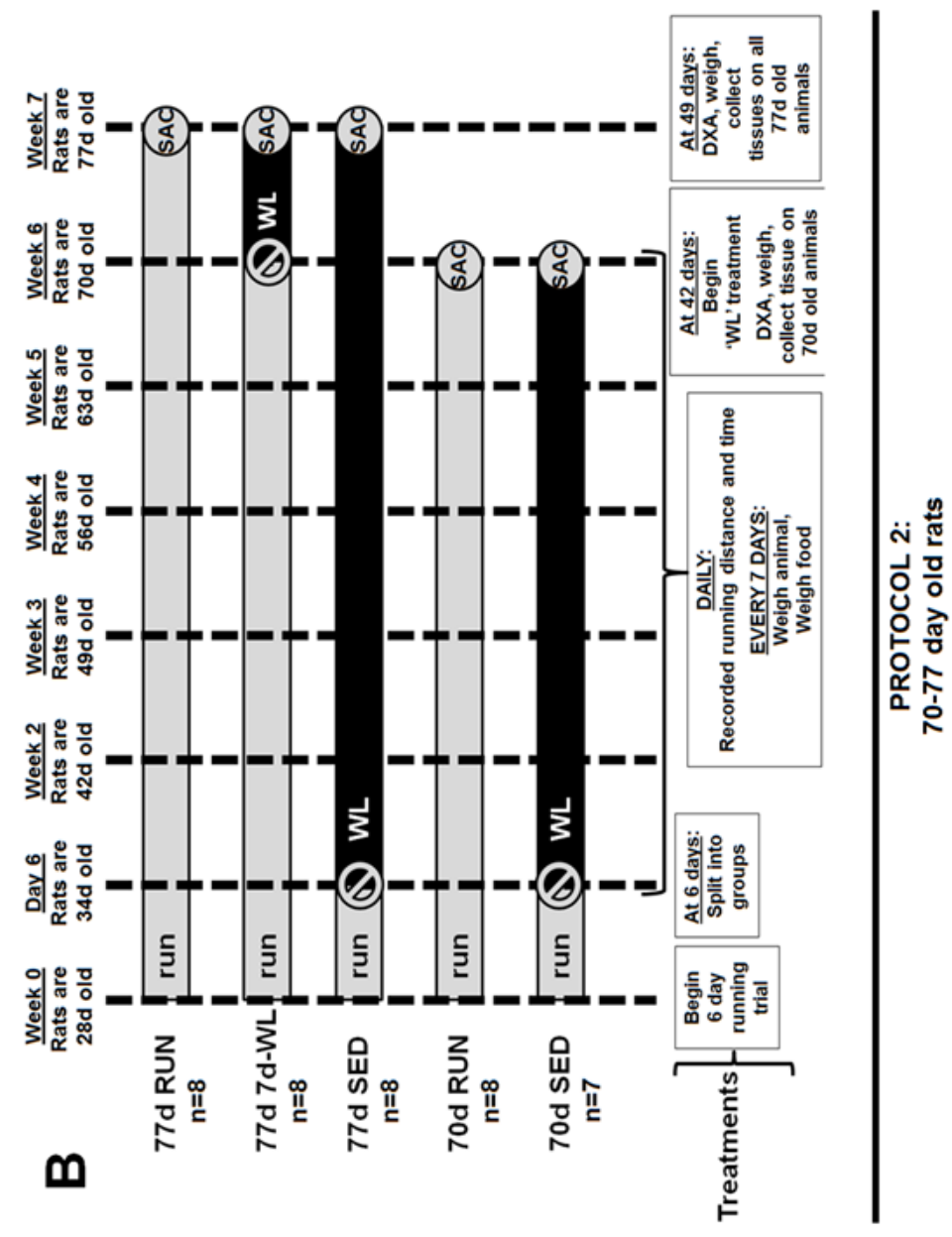

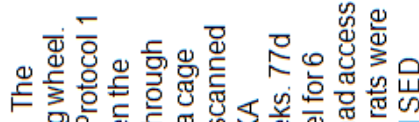

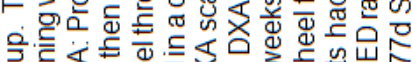

을

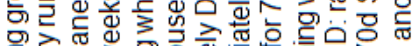

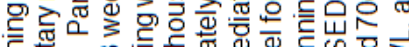

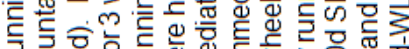

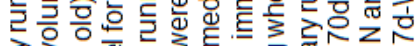

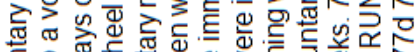

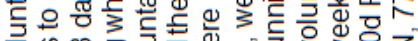

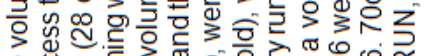

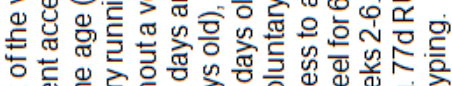

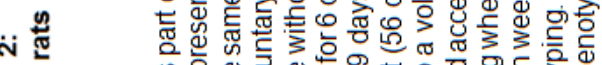

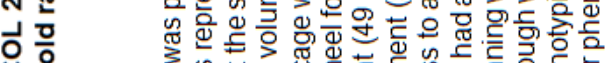

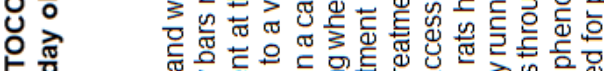

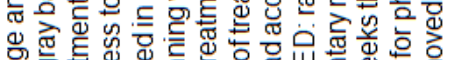

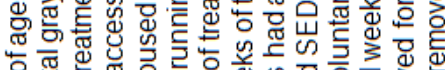

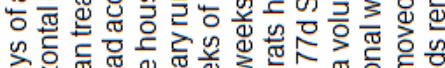

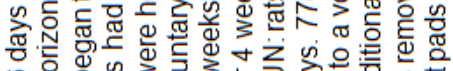

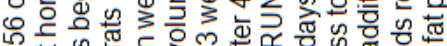

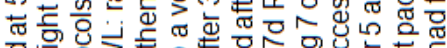

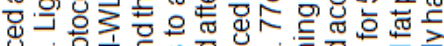

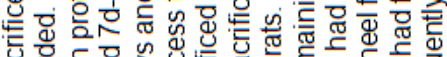

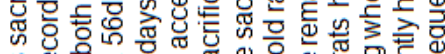
出

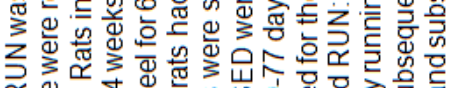

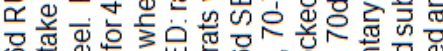
品焉

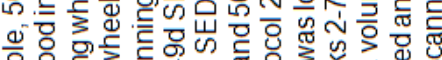

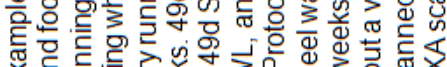

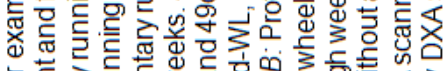

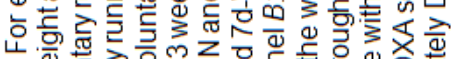

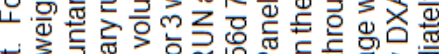

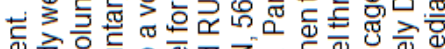

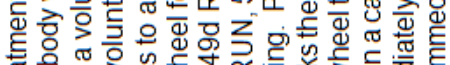

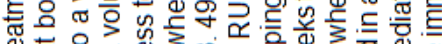

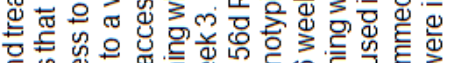

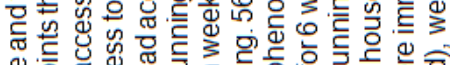

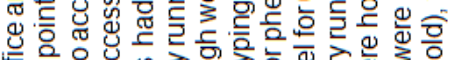

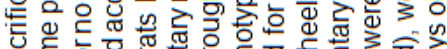

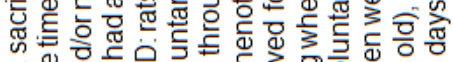

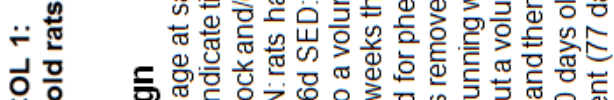

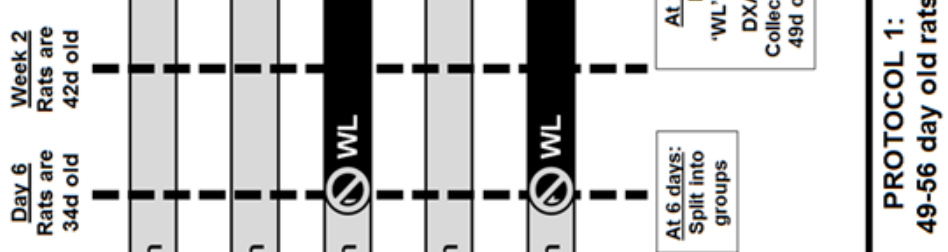

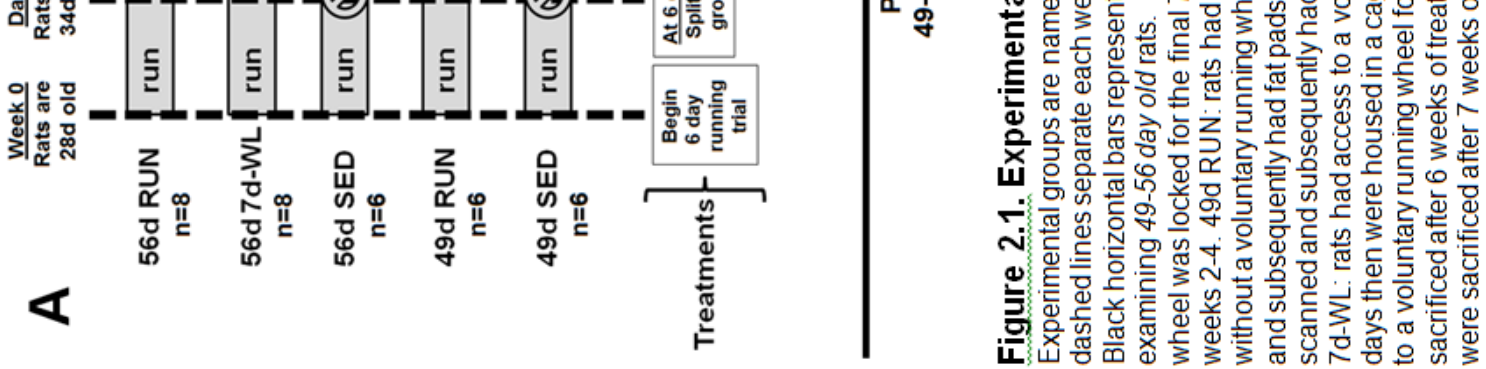


and weighed, while a portion of umbilical/groin SAT was collected. A section of each fat depot was snap-frozen in liquid nitrogen and stored at $-80^{\circ} \mathrm{C}$ for realtime reverse transcriptase polymerase chain reaction (RT-PCR) mRNA analysis and another section was saved for adipocyte sizing.

$R T$ - PCR for adipose tissue mRNA expression patterns

Approximately $100 \mathrm{mg}$ frozen AT was homogenized in $1 \mathrm{ml} \mathrm{RNA} \mathrm{lysis}$ buffer (Tri Reagent - Sigma) using a high-speed shaking device with a stainless steel bead at $30 \mathrm{~Hz}$ for 1 min (Tissuelyser LT, Qiagen, Valencia CA). RNA was isolated using the method according to manufacturer's instructions (Tri Reagent - Sigma, St. Louis, MO) and lack of RNA degradation was verified on a 1\% agarose gel. $1 \mu \mathrm{g}$ of RNA was DNase-treated using DNase I (Thermo Scientific, Glen Burnie, MD) and reverse transcribed using a High Capacity cDNA Reverse Transcription kit (Applied Biosystems, Carlsbad, CA). Gene-specific primers were constructed (Table 2.1) and primer efficiency curves were produced for all genes. Primer efficiencies ranged between $90-110 \%$ for all genes. Twenty-five ng of cDNA was assayed (NanoDrop 1000 -ThermoScientific) from each sample in duplicate for the targets listed in Table 2.1 using SYBR green chemistry (Power SYBR green Mastermix - Applied Biosystems). mRNA expression values are presented as $2^{\triangle \Delta C T}$ whereby $\triangle C T=B 2 M C T$ - gene of interest $C T$ and were normalized to 49d RUN. 


\begin{tabular}{|c|c|c|}
\hline TARGET & FORWARD $\left(5^{\prime} \rightarrow 3^{\prime}\right)$ & REVERSE $\left(5^{\prime} \rightarrow 3^{\prime}\right)$ \\
\hline \multicolumn{3}{|l|}{ Inflammatory } \\
\hline $\mathrm{TNF} \alpha$ & CCCAGAAAAGCAAGCAACCA & CCTCGGGCCAGTGTATGAGA \\
\hline IL-6 & AGTGGCTAAGGACCAAGACCATCCA & GGCATAGCACACTAGGTTTGCCGAG \\
\hline MCP-1 & CTGTCTCAGCCAGATGCAGTTAA & AGCCGACTCATTGGGATCAT \\
\hline \multicolumn{3}{|l|}{ Hypoxia } \\
\hline HIF1 $\alpha$ & GCTGCCTCTTCGACAAGCTT & CGCTGGAGCTAGCAGAGTCA \\
\hline VEGF & GGAGGATGTCCTCACTTGGA & CAAACAGACTTCGGCCTCTC \\
\hline \multicolumn{3}{|l|}{ Adipokine } \\
\hline Leptin & CGCTTCCTGTGGCTTTGGTC & CCСACTGCGTGTGTGAAATG \\
\hline Adiponectin & TCCСTCCACCCAAGGAAACT & GGCCCGGTATCCCATTG \\
\hline \multicolumn{3}{|l|}{ Proliferation } \\
\hline Cyclin A1 & TGCCTGAGTGAGCTGCATAAA & TGCTTGCTGCGGTCGAT \\
\hline CDK2 & ACTAAACCAGTGCCCCACCTT & ACCACAGGTGAAGAGGGCTTT \\
\hline \multicolumn{3}{|c|}{ Differentiation } \\
\hline PPAR $\gamma$ & AAGTCCCAGTCGCTGACAAAGT & TGTCAGATTTTTTTTCCCCTCAAA \\
\hline $\mathrm{C} / \mathrm{EBP} \alpha$ & ATAAAGCCAAACAGCGCAAC & CGGTCATTGTCACTGGTCAA \\
\hline \multicolumn{3}{|c|}{ Housekeeping } \\
\hline $\mathrm{B} 2 \mathrm{M}$ & TGCTACGTGTCTCAGTTCCA & GCTCCTTCAGAGTGACGTGT \\
\hline
\end{tabular}

TNF-a: Tumor Necrosis Factor-alpha; IL-6: Interleukin-6; MCP-1: Monocyte Chemoattractant Protein-1; HIF1a: Hypoxia-Inducible Factor-1 alpha subunit; VEGF: Vascular Endothelial Growth Factor; CDK2: CyclinDependent Kinase 2; PPARy: Peroxisome Proliferator-Activated Receptor gamma; C/EBPa:

CCAAT/enhancer binding protein alpha.

\section{Adipocyte sizing and number}

Adipocyte sizing was performed on EAT in Protocol 1 and three visceral AT depots: OMAT, EAT, and PRAT in Protocol 2 using techniques described previously [60]. Briefly, three 60-80 mg adipose tissue fragments per depot per animal were placed in a glass scintillation tube containing $3 \mathrm{ml}$ Krebs-RingerHEPES buffer (KRBH; $130 \mathrm{mM} \mathrm{NaCl}, 4.7 \mathrm{mM} \mathrm{KCl}, 1.2 \mathrm{mM} \mathrm{KH}_{2} \mathrm{PO}_{4}, 10 \mathrm{mM}$ HEPES, $1 \mathrm{mM} \mathrm{CaCL}_{2}, 1.2 \mathrm{mM} \mathrm{MgSO}_{4}, 0.25 \mathrm{mg} / \mathrm{ml}$ free fatty acid-free $\mathrm{BSA}, \mathrm{pH}=$ 7.4). Fragments were subsequently minced into $\sim 1 \mathrm{~mm}^{3}$ pieces, incubated with 0.920 Wunsch units of Liberase Blendzyme (Roche) in a $37^{\circ} \mathrm{C}$ incubator at 100 rpm for 1 hour, and then transferred through a sterile $200 \mu \mathrm{m}$ nylon mesh (Sefar America, Kansas City, MO) into a $15 \mathrm{ml}$ conical tube. After centrifugation at 100 rpm for 1 minute, the adipocytes were loosely packed at the top of the liquid and the bottom layer of liquid was removed using a syringe and needle. The 
adipocytes were washed with $\sim 5 \mathrm{ml} \mathrm{KRBH}$ then resuspended in $5 \mathrm{ml} \mathrm{KRBH}$ for every $1 \mathrm{ml}$ of loosely packed adipocytes. A random sample of the adipocytes was viewed with a Nikon Eclipse E600 microscope and photographed by an Olympus DP72 camera. The diameter of $>300$ adipocytes were measured using DP2-BSW v2.1 software. The mean diameter and size distribution were calculated for each adipocyte depot.

The number of adipocytes per depot was calculated with the mass of the fat depot, a density conversion factor (0.915 ng lipid/1 pl), and average diameter of adipocytes [97]. Briefly, the average cell mass was calculated by: average cell size $(\mathrm{pl}) \times 0.915(\mathrm{ng} \mathrm{lipid} / 1 \mathrm{pl}) \times \mathrm{g} / 10^{9} \mathrm{ng}$. The number of cells per depot was calculated as: mass of adipose depot (g) / average mass of adipocyte (g). Reliability of this method is described in detail [97] and has been used by others [98]. There are two ways to present adipocyte cellularity: number of cells per depot and number of cells per gram of adipose tissue. The rationale for presenting adipocyte cellularity as "number of cells per depot" is that an underlying question was whether visceral adipose tissue was expanding by hypertrophy or hyperplasia. To address this, it was necessary to calculate an estimate of total adipocytes in a depot. The problem with expressing adipocyte cellularity as "adipocytes per gram of adipose tissue" is that, while it addresses cell density, it does not prove hyperplasia. For example, if we see an increase in cell density (more adipocytes per gram of tissue), we can conclude that either, 1) hyperplasia is occurring and new adipocytes are being formed and causing the increased density or 2) lipolysis is occurring and current adipocytes are shrinking 
and causing the increased density. Because of this uncertainty, adipocyte cellularity is expressed as "number of adipocytes per depot."

\section{Statistical analysis}

Outcome measures for between-group or within-group comparisons (adipose depot mass, adipocyte size, adipocyte size distribution, and RT-PCR) were analyzed with a one-way analysis of variance. For outcome measures that had the same measures on the same animal (weekly body weight, daily distance run, and weekly food intake), a two-way analysis of variance was performed (group $x$ time). Significant main effects $(p<0.05)$ were followed up with Fisher LSD post hoc comparisons. Sigmaplot 12.0 (San Jose, CA) was used for all statistical analyses. Values are reported as means \pm SE, and significance for all tests was set at $p<0.05$. Exceptions to these statistical methods are stated in figure legends.

\section{RESULTS}

Daily running distances. RUN only (77d RUN), and RUN followed by $7 \mathrm{~d}-$ WL groups (hereafter referred to as $7 d-W L$ ) ran similar distances during the treatment period (Fig. 2.2). All 70-77 day old groups (70d RUN, 77d 7d-WL, and $77 \mathrm{~d} R \mathrm{RN}$ ) appeared to reach a plateau in nightly running distance by the $4^{\text {th }}$ week of running. 

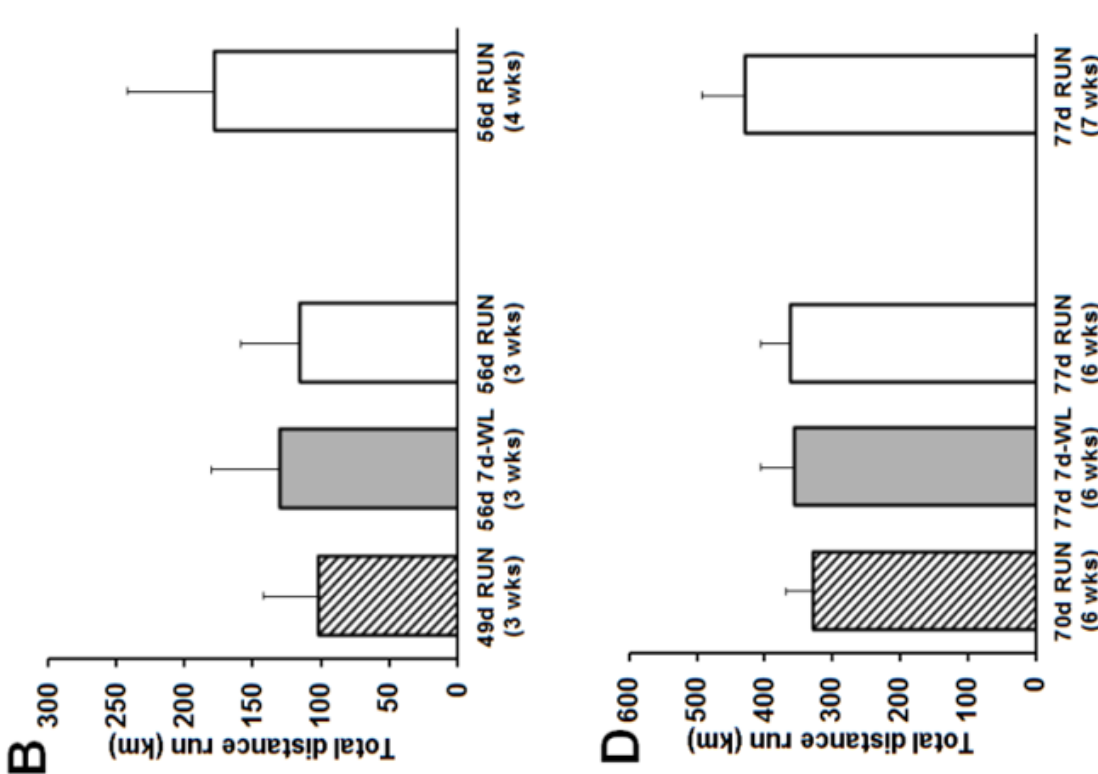

㞼

솔웡

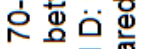

됭

ฏ。

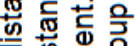

응

을

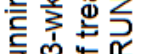

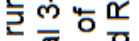

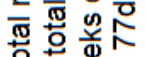

음

둥

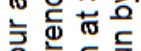

웋동

京要

능

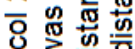

구음

๓

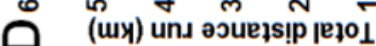

흔 迹온

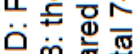

ํํำ

둥 흘

0 \%

웅+人올

드은응

므웜

ต่

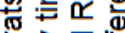

능 중

응 कํํㅇำ

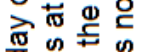

을 을

ํํㅇํํㄴ

ชํํㄹ

동

ฮ)

워음

응 은

잉

엉

동 몽

능을

오워

응 항

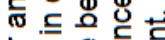

這

둥 엄

品离产美

牙琣

응
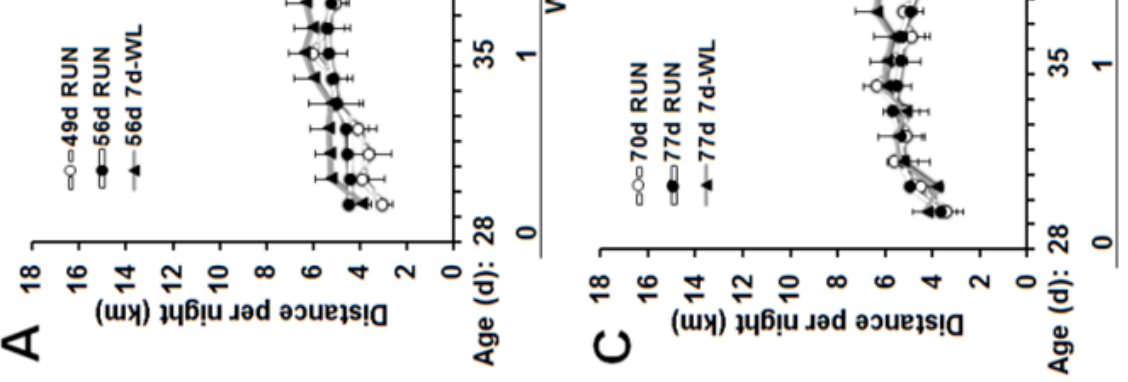

응 ㅎ⿺ㄴ

a 0

的

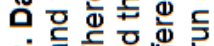

งู के

งषิ

능

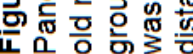


Weekly body weights. Gains in body weight expressed as grams gained per week for all groups are consistent through 49 days of age in both age groups of rats then decrease through 77 days of age (Fig. 2.3C and 2.3D). Importantly, in 49-56 day old rats, WL was performed before any notable drop in grams gained per week. In contrast, in 70-77 day old rats, WL was performed at an age where grams gained per week had significantly decreased. Body mass growth during WL (56d 7d-WL) between the ages of 49-56 days paralleled growth in the 56d RUN and 56d SED groups (Fig. 2.3A and 2.4A). However in three-week older rats, body mass growth during WL (77d $7 d-W L)$ no longer paralleled $77 d$ RUN and 77d SED (Fig. 2.3B and 2.4E).

Body mass, DXA lean body mass, DXA fat mass, and DXA percent body fat during wheel lock. WL rats showed an increased rate of fat mass gain and body fat \% gain compared to RUN rats in both 49-56 day old rats (Fig. 2.4C \& 2.4D, different capital letters represent different slopes) and 70-77 day old rats (Fig. 2.4G \& 2.4H, different capital letters represent different slopes), so that the slope of gain during WL was similar to SED. It is notable that the slope of \% body fat was significantly less between both $49-56$ days and 70-77 days in $56 \mathrm{~d}$ RUN and 77d RUN versus the respective upward slopes of WL (Fig. 2.4C and $2.4 \mathrm{~F}$ ), and gains in \% body fat were attenuated in $56 \mathrm{~d}$ RUN rats compared to $56 \mathrm{~d}$ 7d-WL and 56d SED. WL did not affect lean body mass gains in either age group (Fig. 2.4B \& 2.4F). 


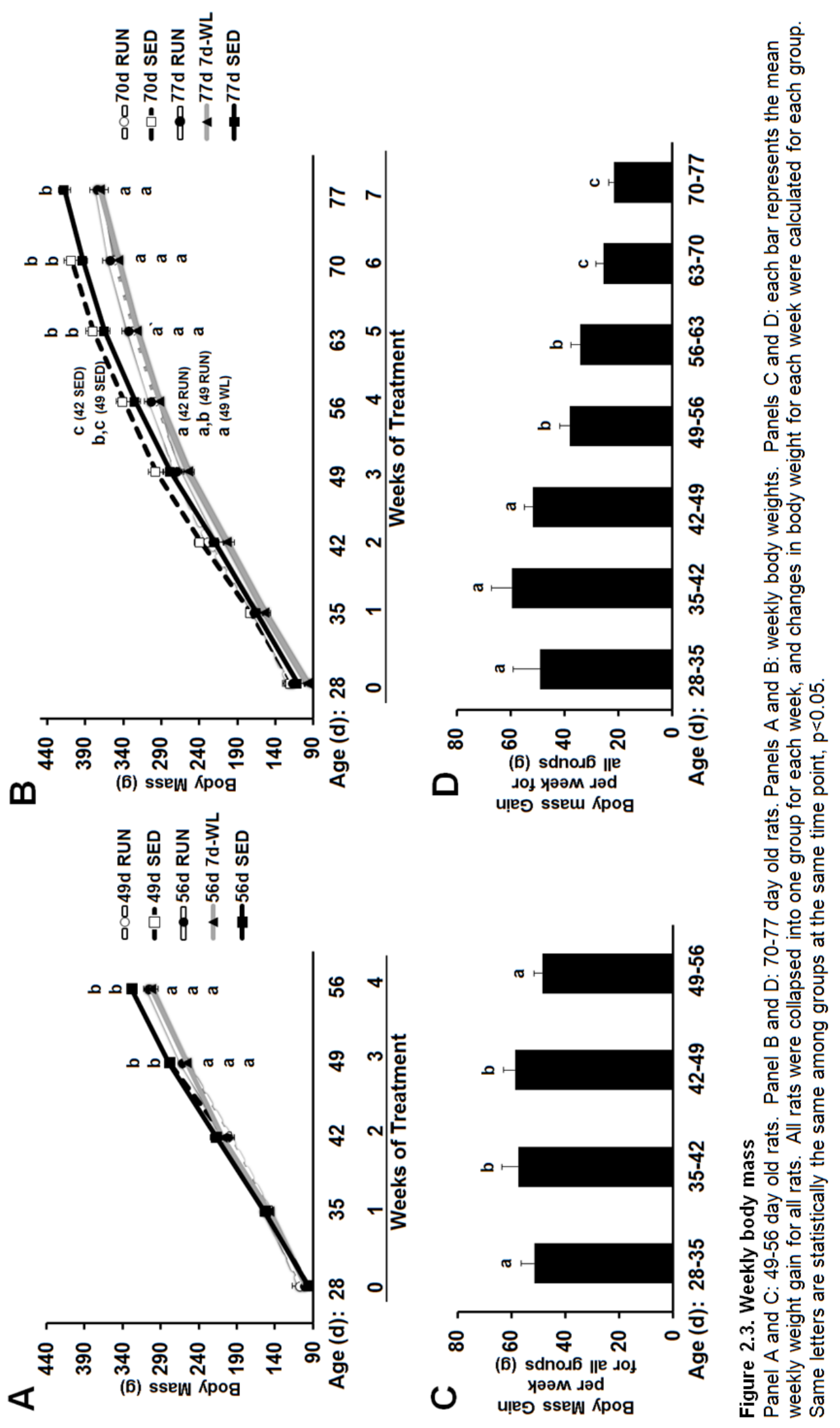




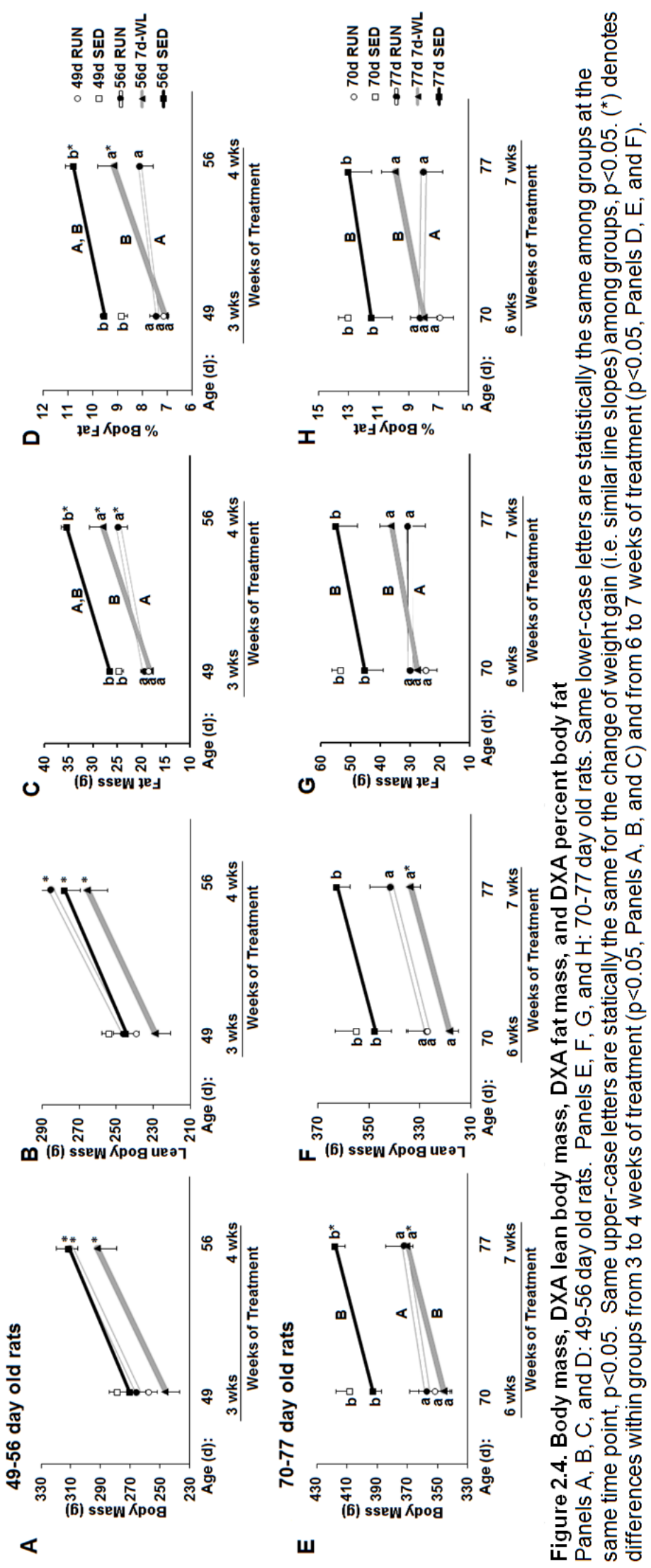


Weekly food intake in 70-77 day old rats. Apart from week 7 , there was no activity effect on weekly food intake (Fig. 2.5A). All groups reached a relative steady-state in food intake by week 4 and, with the exception of $77 d 7 d-W L$, remained constant through week 7 . Three days after $W L, 77 d 7 d-W L$ rats decreased food intake and maintained this level for the remaining four days (Fig. $2.5 \mathrm{~B})$, indicating a rapid response in appetite to decreased activity as food intakes were tending to show progressive drops already on $1^{\text {st }}$ and $2^{\text {nd }}$ days of WL.

Feed efficiency in 70-77 day old rats. 77d SED rats had greater weekly feed efficiency during weeks 3, 5, and 7 compared to RUN rats, but WL increased weekly feed efficiency to SED levels (Fig. 2.6A), despite a rapid decrease in food intake (Fig. 2.5B). Day-to-day feed efficiency during WL showed a transient one-day increase in feed efficiency at day 1 of WL followed by an abrupt decrease and plateau in feed efficiency to pre-WL levels from days 2-7 days of $\mathrm{WL}$ (Fig. 2.6B), which highlights the impact a sudden decrease in activity has on energy homeostasis. 


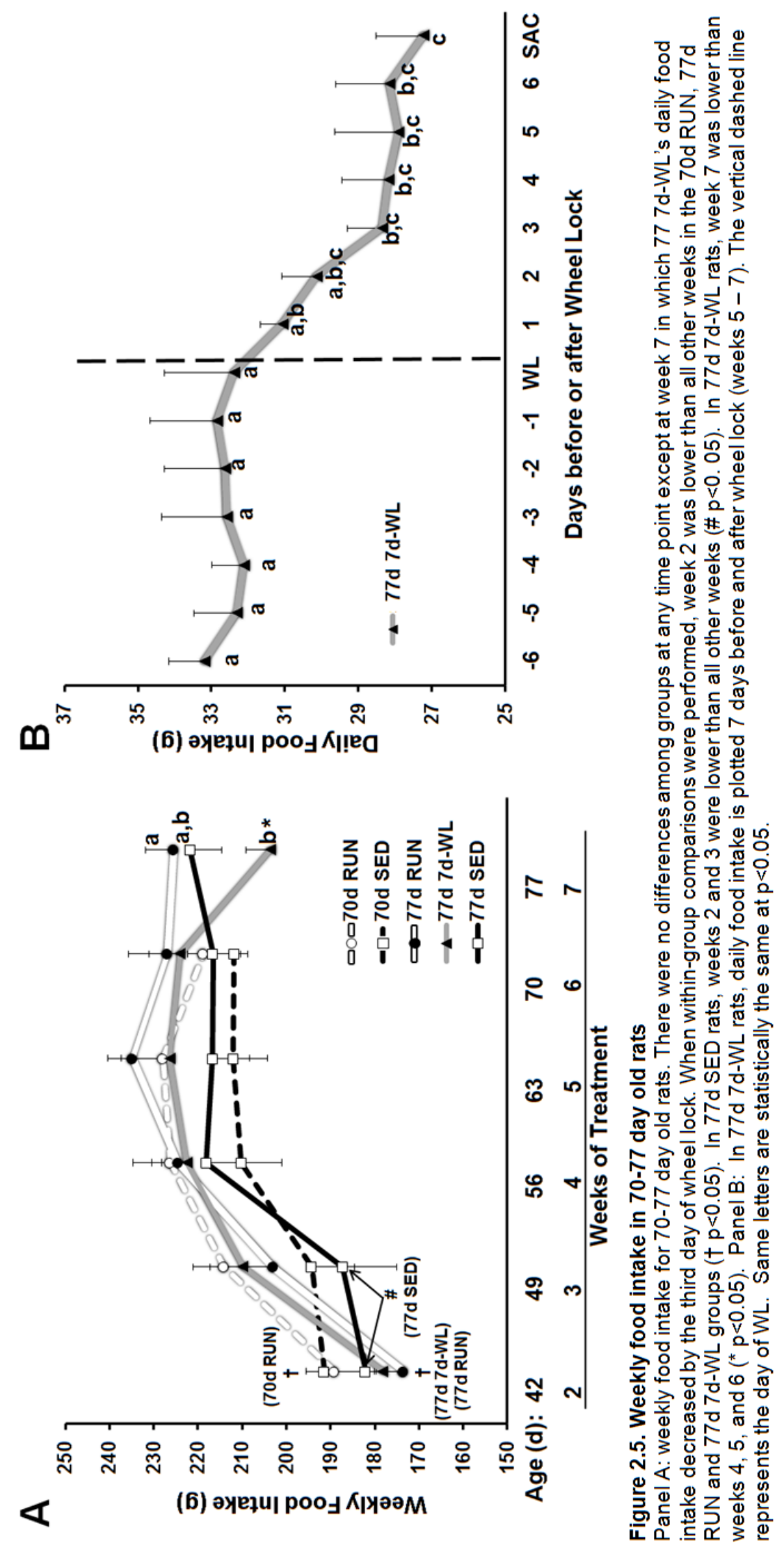




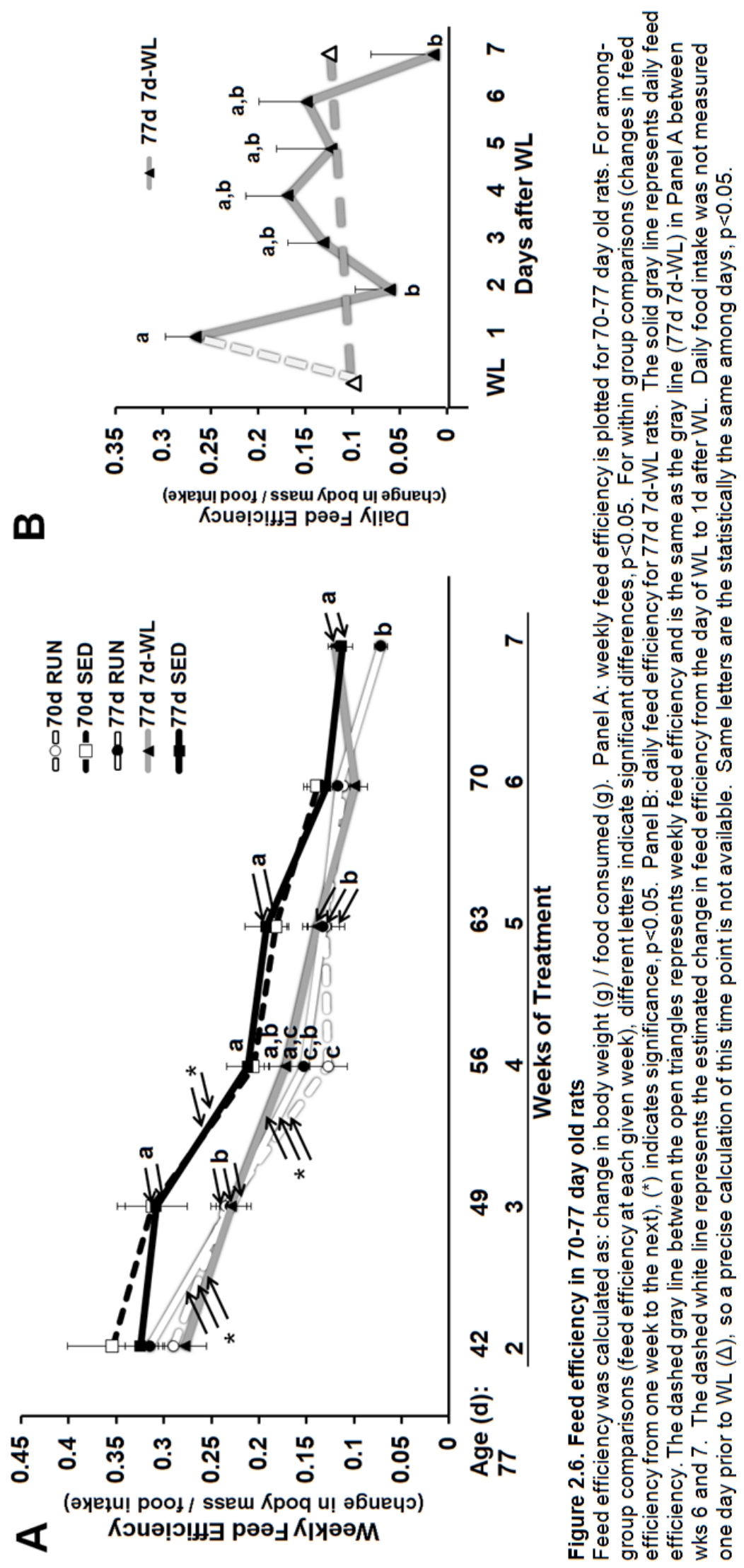


Adipose tissue depot mass. 49-56 day old rats' adipose tissue mass increased independent of activity level (49d RUN < 56d RUN and 49d SED $<56 d$ SED) (Fig. 2.7A-C), suggesting that these rats are 'growing' in terms of body mass and adipose depot mass whether they are physically active or not. WL did not affect this increase $(56 d \mathrm{RUN}=56 \mathrm{~d} W L)$ in all three depots (Fig. 2.7A-C). In contrast, 70-77 day old rats' adipose tissue mass has reached a plateau and did not increase between 70-77 days (70 RUN = 77 RUN and 70 SED = 77 SED) (Fig 2.7D-F), suggesting that these rats are not 'growing' in terms of adipose depot mass as rapidly as $49-56$ day old rats. WL induced mass gains between 70-77 days (77 RUN < $77 \mathrm{WL}$ ) in EAT and PRAT (Fig 2.7D and 2.7E).

Adipocyte diameter. Adipocyte diameter data trends follow depot mass trends. 49-56 day old rats' EAT adipocytes appear to expanding via hypertrophy independent of activity level (49d RUN < 56d RUN and 49d SED < 56d SED) (Fig. 2.8 A), and WL did not affect this (56d RUN = 56d 7d-WL) (Fig 2.8 A). 7077 day old rats' adipocyte diameter reached a plateau (70d RUN $=77 d$ RUN and 70d SED = 77d SED) (Fig 2.8B-D). WL increased adipocyte diameter (77d RUN $<77 \mathrm{~d} 7 \mathrm{~d}-\mathrm{WL}$ ) in EAT and PRAT (Fig 2.8E and 2.8F). 

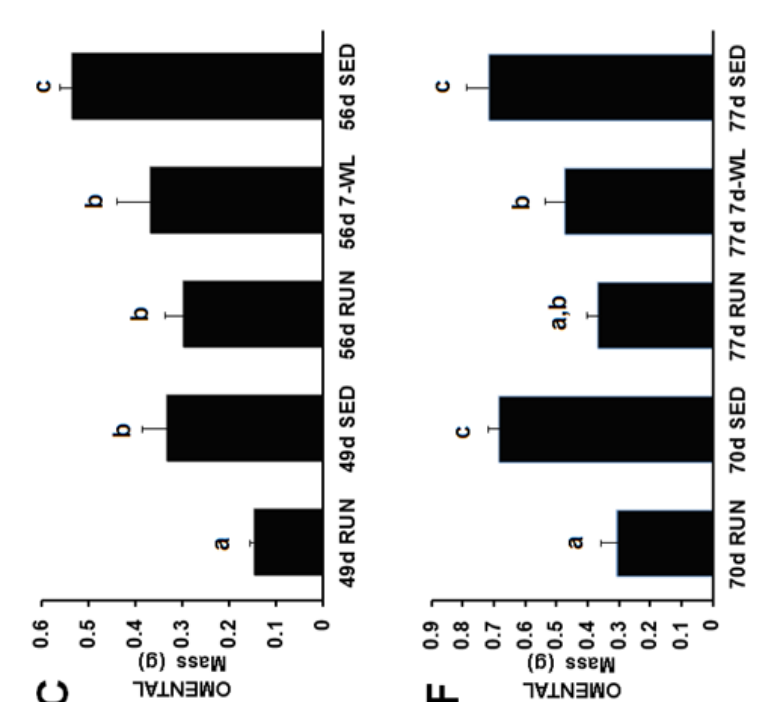

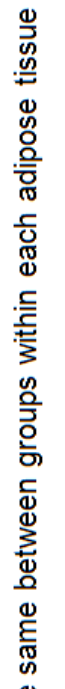
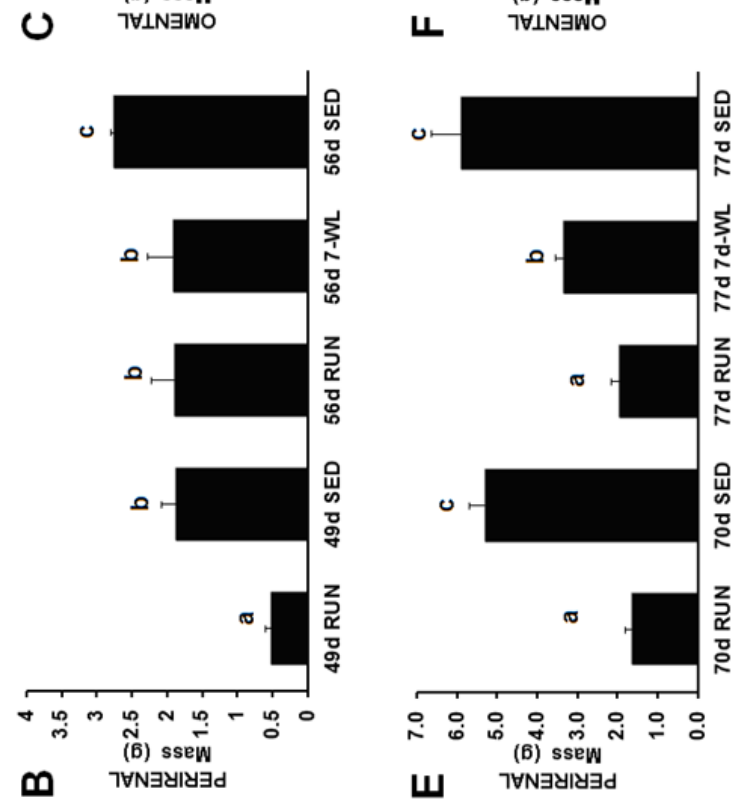

$\stackrel{\Phi}{\mp}$

‡

章

ญ

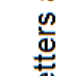

है

(

苗

흥

तु

Tे
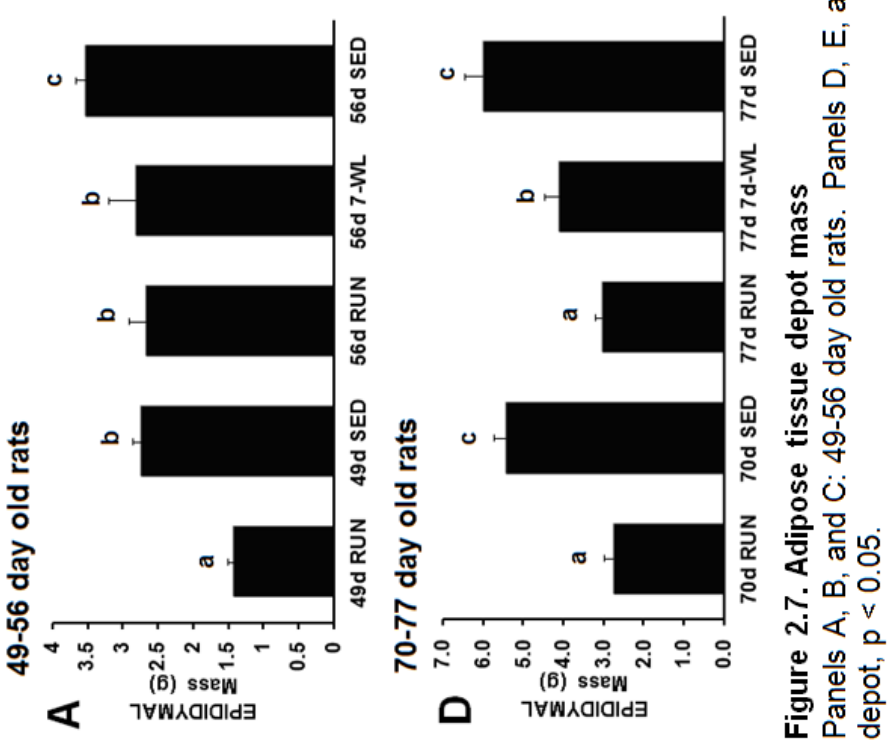
Total Adipocyte Number

in depot ( $\times 10^{6}$ cells)

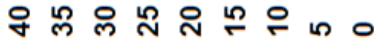

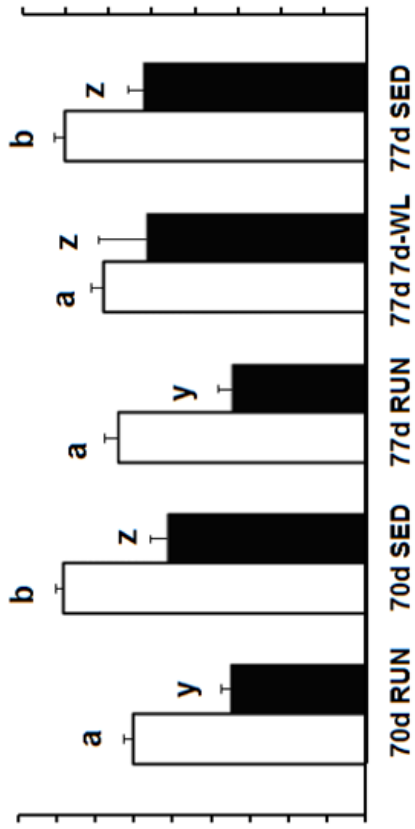

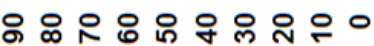

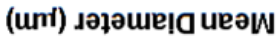

$7 \forall N \exists$ GIY $\exists d$

U

Total Adipocyte Number in depot $\left(\times 10^{6}\right.$ cells)

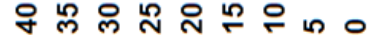

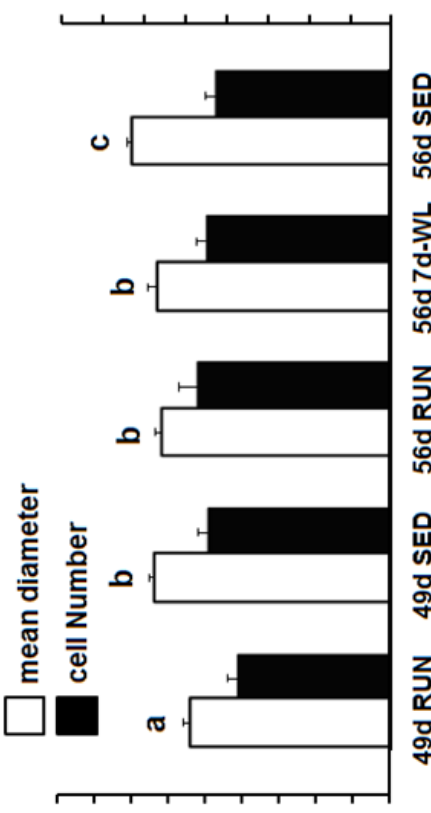

용ㅇㅇ용유잉응

(uni) sәјәше व uеәw

$\varangle \quad 7 \forall$ W
Total Adipocyte Number

in depot $\left(\times 10^{6}\right.$ cells $)$

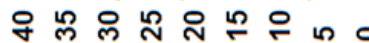

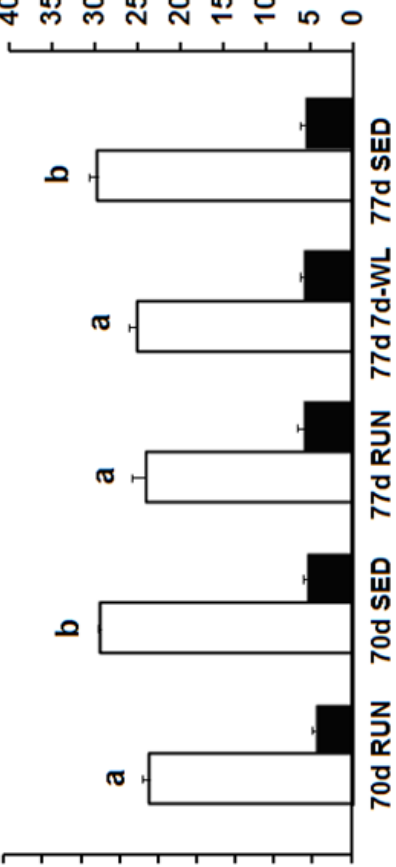

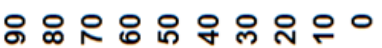

(uni) دәฺәแе! वеәW

$7 \forall \perp N \exists W O$

Total Adipocyte Number

in depot $\left(\times 10^{6}\right.$ cells $)$

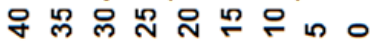
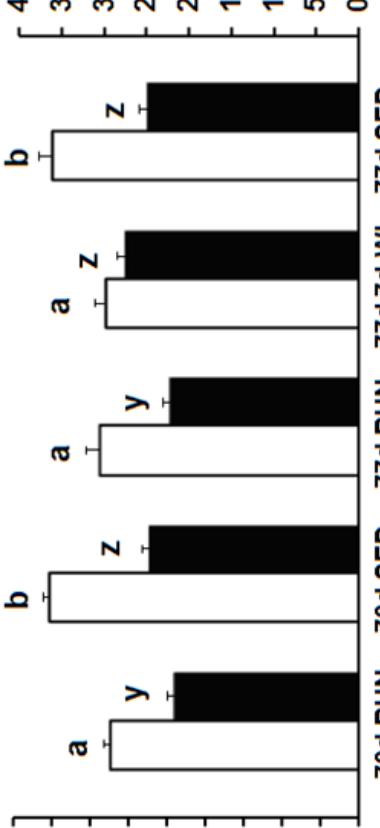

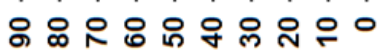

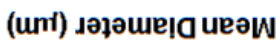

7甘W人alald $\exists$
○்

首 $N$

$0>$

๓

$\overline{\text { ๘ }}$

ลั $\frac{\overline{1}}{\mathrm{D}}$

힌

흥 음

तั่

꿍 듀

ํํㄹ

守 뜬

宅 을

ब 잉

กั

ั艹்

웡형

d 응

艺

(ᄈ)

.

๕ั๊ 음

ह

돓

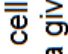

월

荧

응

玉 告 气

๘ 도은

틀든

อ ญ

凹 힝

중인

응

는 क

ธิ

픙 흥

든 $\frac{0}{4}$

๘亦

莎的

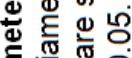

हํㅡㅇ

㟧

웡눙

艺

응 ह

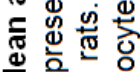

는 은흥음

$\infty$ 엥

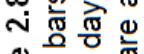

․ㅗ음

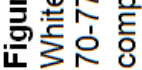


Total adipocyte number in depot. WL did not affect total adipocyte number in EAT in these 49-56 day old rats (56d RUN $=56 d$ 7d-WL), emphasizing that the increased total number of adipocytes between $49 d$ RUN and $56 d 7 d-W L$ was not due to WL, it was due to aging. In 70-77 day old rats, total adipocyte number reached a plateau in all three depots $(70 \mathrm{RUN}=77 \mathrm{RUN}$ and $70 \mathrm{SED}=$ 77 SED) (Fig 2.8 B-D). WL increased total adipocyte number in EAT and PRAT (77 RUN < $77 \mathrm{WL}$ ) in EAT and PRAT (Fig 2.8B and 2.8C).

Taken together, the adipocyte depot mass, adipocyte diameter, and total adipocyte number in a depot data suggests that 1) adipose tissue in 49-56 day old rats is still expanding with 7 days of age while it is not in 70-77 day old rats. 2) WL does not alter this adipose tissue expansion in 49-56 day old rats, while it increases depot mass and total adipocyte number in 70-77 day old rats.

Adipocyte size distribution. Activity and age affect adipocyte size distribution, and WL alters the adipocyte size distribution in EAT and PRAT depots. In 49-56 day old rats EAT, there is a rightward shift in the $56 \mathrm{~d} 7 \mathrm{~d}-\mathrm{WL}$ group that parallels the shift seen in the 56 RUN group, but there are two distinct differences: 1) there is a 'flattening out' of the $56 \mathrm{~d} 7 \mathrm{~d}-\mathrm{WL}$ curve such that the peak is lower than $56 \mathrm{RUN}$, and 2) $56 \mathrm{~d} 7 \mathrm{~d}-\mathrm{WL}$ has more adipocytes in the $40 \mu \mathrm{m}$ category than $56 \mathrm{~d}$ RUN (Fig. 2.9A), a finding that may indicate the formation of smaller adipocytes via hyperplasia is occurring. In contrast, the rightward shift in EAT is absent in 70-77 day old rats within the $77 d \mathrm{~d}$ d-WL group, but there are more 'small' adipocytes at 20-, 30-, and 40- $\mu \mathrm{m}$ categories (Fig. 2.9B); again, this 
being a finding that may indicate the formation of smaller adipocytes via hyperplasia is occurring. Similar to $70-77$ day old rats' EAT, PRAT also shows a 'flattening out' of the $77 \mathrm{~d} 7 \mathrm{~d}-\mathrm{WL}$ curve with more 'smaller' adipocytes at 30 - and 40- $\mu$ m categories (Fig. 2.9C). 77d 7d-WL OMAT shows a rightward shift, but little to no flattening out (Fig. 2.9D).

Apart from the WL effect described above, there was a physical activity effect and age effect on adipocyte distribution. In both the 49-56 day old rats and 70-77 day old rats, SED rats showed an apparent rightward shift in the peak percentage adipocyte diameter such that for all age-matched groups RUN $<$ SED (Fig. 2.9). Objectively, the mean diameter was greater in SED compared to RUN. Subjectively, the curve is visually shifted to the right in SED compared to RUN. In the 49-56 day old rats, a rightward shift in the peak percentage from 49 days of age to 56 days of age was observed in both RUN and SED so that 49 RUN $<56$ RUN and 49 SED < 56 SED (Fig. 2.9A), implying that at this age, adipocytes are still in a growth phase, and this growth occurs independent of activity level. In contrast, this rightward shift is not present in the 70-77 day old rats: $70 d$ RUN $=77 d$ RUN and 70d SED $=77 d$ SED (Fig. 2.9B-D) implying that the adipocyte growth has reached a steady-state at sexual maturity. 

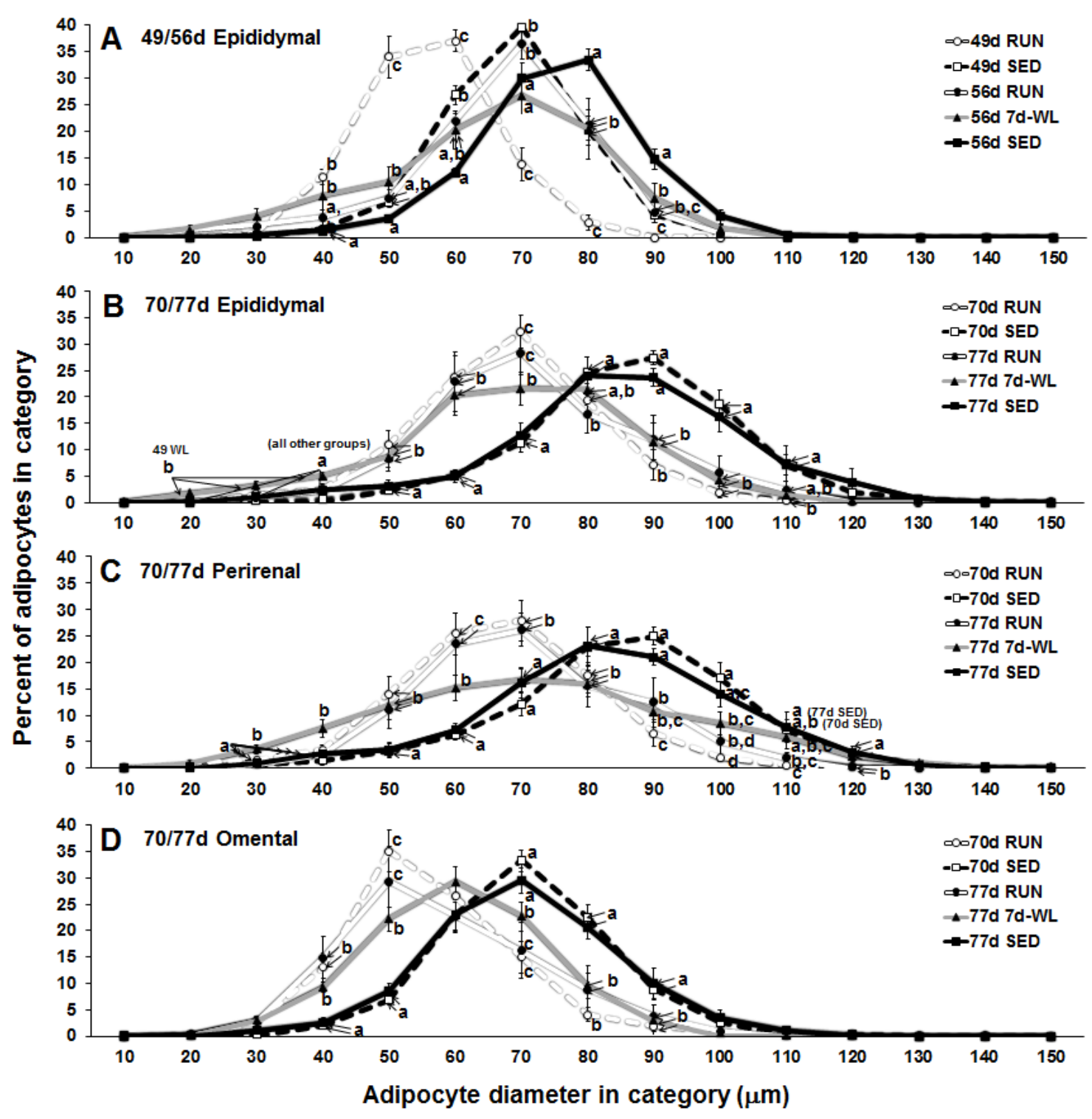

Figure 2.9. Adipocyte size distribution

Adipocyte diameters were classified in 10- $\mu \mathrm{m}$ categories (10: 0.0-9.9 $\mu \mathrm{m}, 20: 10.0-19.9 \mu \mathrm{m}, 30: 20.0-29.9$ $\mu \mathrm{m}$, etc.) and the percentage of total adipocytes in a sample was plotted for each category. At least 300 adipocyte diameters were measured for each tissue sample. Same letters are statistically the same among groups at each category, $\mathrm{p}<0.05$.

WL increased cyclin A1 mRNA expression to SED levels in 70-77 day old rats EAT, PRAT, and SAT, but not in OMAT. There was a physical activity effect on cyclin A1 mRNA levels in 70-77 day old rats such that RUN expressed less cyclin A1 mRNA than SED or 77d 7d-WL in EAT, PRAT, and SAT, but not OAT 
(Table 2.2, Fig. 2.10B). This data suggests that voluntary wheel running may act as a 'brake' that attenuates expression of this proliferation gene in some, but not all fat depots, and removing this 'brake' via extraneous inactivity increases a marker of adipocyte hyperplasia, cyclin A1 [99]. There were no differences in cyclin A1 mRNA in 49-56 day old rats, suggesting that adipose tissue depots are still in the process of expanding and new adipocytes are still being formed regardless of physical activity level. In addition to cyclin A1 mRNA increases, WL increased HIF-1a mRNA to 77d SED levels in EAT and paradoxically decreased IL-6 mRNA of 70d RUN rats in PRAT.
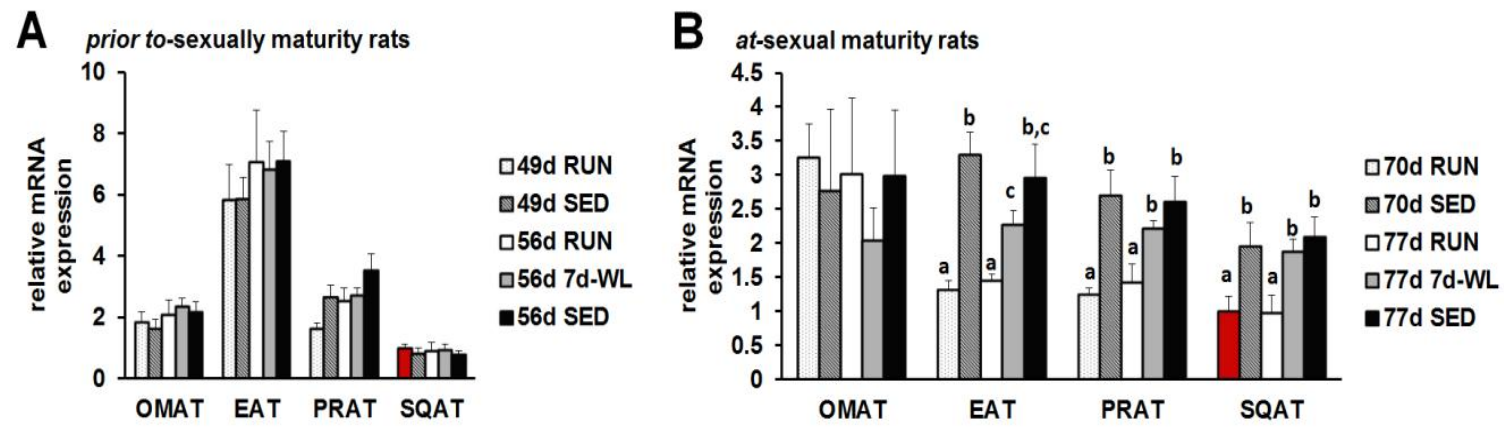

\section{Figure 2.10. mRNA expression of cyclin A1}

Panel A: 49-56 day old rats cyclin A1 mRNA expression was normalized to 1.0 for 49d RUN SQ (red bar). There were no differences between groups within each depot. Panel B: 70-77 day old rats cyclin A1 mRNA expression was normalized to 1.0 for $70 \mathrm{~d}$ RUN SQ (red bar). Different letters indicate significant differences among groups in each depot, $p<0.05$. OMAT = omental adipose tissue; $E A T=$ epididymal adipose tissue; PRAT = perirenal adipose tissue; and SQAT = subcutaneous adipose tissue. Same letters are statistically the same. 


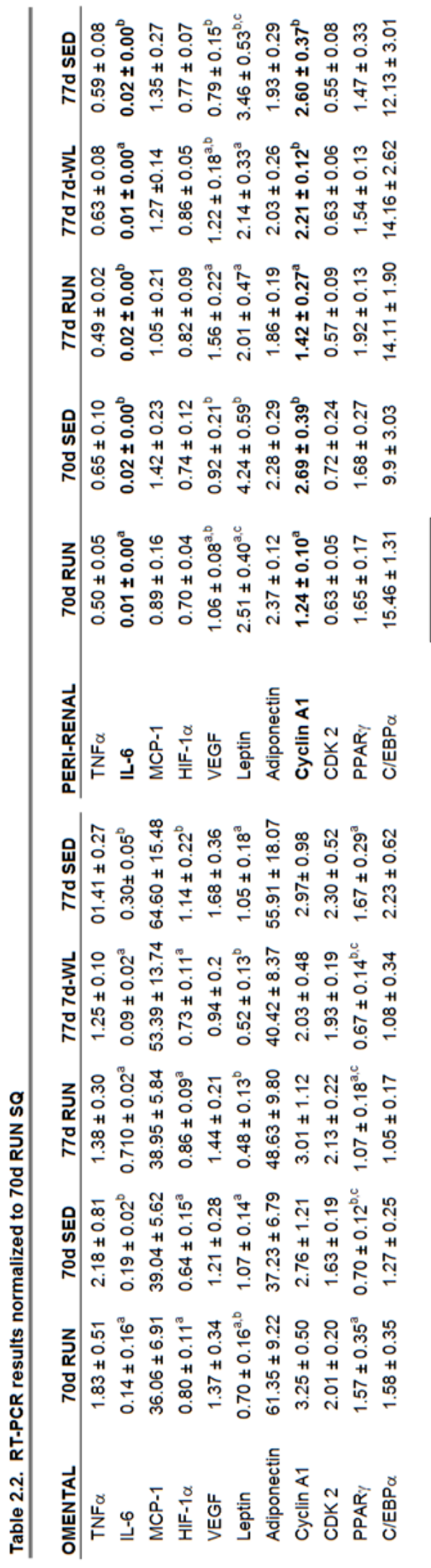

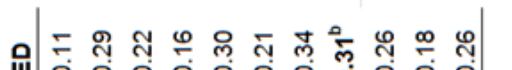
岁

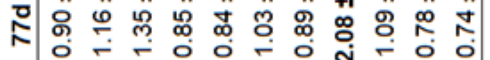

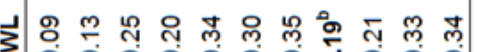

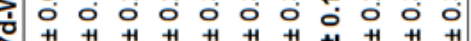

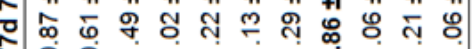

ป

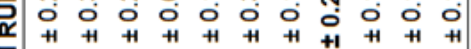

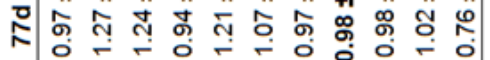

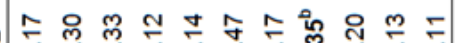
崽算

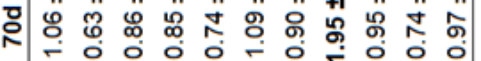

z

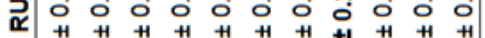

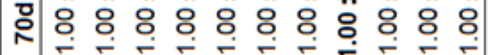

ป

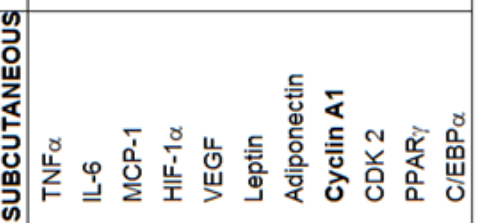

بิنั

ํㅗ원

곡뭉모

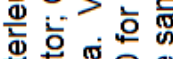

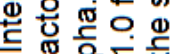

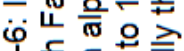

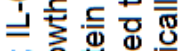

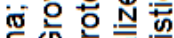

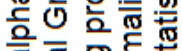

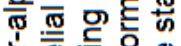

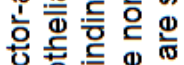

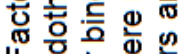
山市市离 븜 岁声 은 흔 듣 过它它

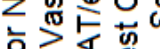
它过岕完

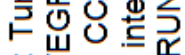
放范范员 ト ํㅡㄱ 屯ั ติ

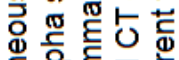
产 음 รูำ 뜽 윽 훙은 ๓

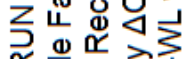

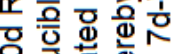

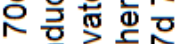
은 흔

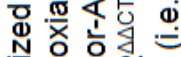

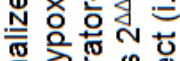

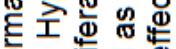

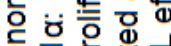
뜬둔 ฮ望 思 흥 흥호 交高高高高 ஸे

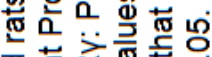

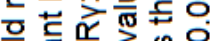

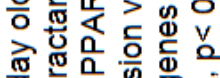

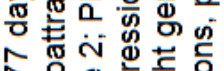
人。ั 잉등

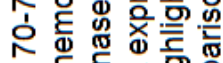
든든흔 을

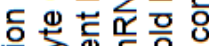

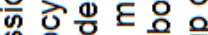
은 든 은 응분 बํ..

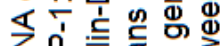

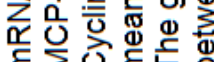




\section{DISCUSSION}

In the current study, we examined two life phases in rats whose ages were selected to mimic a phase of human life where: 1) adipocyte number rapidly increases and then stabilizes $[5,6,10,94]$ and 2) prevalence of U.S. childhood obesity and physical inactivity both simultaneously increase $\sim 50 \%$ from the age ranges of $6-8 \mathrm{yrs}$ to $12-19 \mathrm{yrs}$ of age [see introduction for details and refs [1, 8 , 100]]. We report: 1) compared to 49-56 day old rats, adipose tissue in 70-77 day old rats is more greatly affected by WL and responds by expansion via hyperplasia; 2) in both ages, WL occurs without an increase in inflammatory mRNA expression; 3) only in 70-77 day old rats did 7d-WL increase cyclin A1 mRNA above RUN levels, reaching the higher sedentary levels in EAT, PRAT and SAT (Fig. 2.10). We thus propose for the first time to our knowledge the notion that the type of adipocyte growth mechanism (i.e., hypertrophy or hyperplasia) is dependent on stage of growth during WL.

Rate of growth decreases between 49-56 days of age.

The two ages of rats exhibited different growth accretions, forming a novel, two-phase process dependent on pre-pubertal age (Fig. 2.11). 49-56 day old rats appeared to show no effect of WL in many of our outcome measures (Table 2.3). We observed a decrease in the increment of weekly gains in absolute body mass gains beginning around 49-56-day age interval (Fig. 2.3). This data suggests that 49-56 day old rats are in a more rapid growth phase and are near to an inflection point indicating a new phase of slower growth is 
beginning. This is important because the progressively slower gain in body mass starting at 49 days old may be associated with the previously reported shutting down of adipocyte proliferation with maturation in sedentary rats [10]. Importantly, the 49-56 day old rats' data was collected at 49 and 56 days of age, essentially at the end of the rapid growth phase, while in the 70-77 day old rats' data was collected at 70 and 77 days of age, three weeks into the progressively slower growth increments. Together, these observations set a unique two-phase construct upon which we examined for any co-clustering of directional changes or of no change.

Table 2.3. Summary of outcome measures

\begin{tabular}{|c|c|c|}
\hline MESAURE & GROUP & \\
\hline \multirow{2}{*}{\multicolumn{3}{|c|}{ Body Mass }} \\
\hline & & \\
\hline Absolute gain in last 7 days & no difference & WL gained mass \\
\hline Rate of gain in last 7 days & no difference & WL > RUN \\
\hline \multicolumn{3}{|l|}{ Lean Mass } \\
\hline Absolute gain in last 7 days & no difference & WL gained mass \\
\hline Rate of gain in last 7 days & no difference & no difference \\
\hline \multicolumn{3}{|l|}{ Fat Mass } \\
\hline Absolute gain in last 7 days & no difference & no difference \\
\hline Rate of gain in last 7 days & WL > RUN & WL > RUN \\
\hline \multicolumn{3}{|l|}{$\%$ Body Fat } \\
\hline Absolute gain in last 7 days & $W L$ gained $\% B F$ & no difference \\
\hline Rate of gain in last 7 days & WL > RUN & $W L>R U N$ \\
\hline \multicolumn{3}{|l|}{ Depot Mass } \\
\hline EAT & no difference & 77d 7d-WL > 77d RUN \\
\hline PRAT & no difference & $77 \mathrm{~d} 7 \mathrm{~d}-\mathrm{WL}>77 \mathrm{~d}$ RUN \\
\hline OAT & no difference & no difference \\
\hline \multicolumn{3}{|l|}{ Mean Adipocyte Diameter } \\
\hline EAT & no difference & no difference \\
\hline PRAT & $\mathrm{n} / \mathrm{a}$ & no difference \\
\hline OAT & $\mathrm{n} / \mathrm{a}$ & no difference \\
\hline \multicolumn{3}{|c|}{ Total Number of Adipocytes in Depot } \\
\hline EAT & no difference & 77d 7d-WL > 77d RUN, hyperplasia \\
\hline PRAT & $\mathrm{n} / \mathrm{a}$ & 77d 7d-WL > 77d RUN, hyperplasia \\
\hline OAT & $\mathrm{n} / \mathrm{a}$ & no difference \\
\hline \multicolumn{3}{|l|}{ Adipocyte Distribution } \\
\hline EAT & $\begin{array}{l}\text { WL flattened curve and } \\
\text { had more adiocytes in the } 40-\mu \mathrm{m} \text { categories }\end{array}$ & $\begin{array}{l}\text { WL flattened curve and } \\
\text { had more adipocytes in the } 20-, 30-, \text { and } 40-\mu \mathrm{m} \text { categories }\end{array}$ \\
\hline PRAT & $n / a$ & $\begin{array}{l}\text { WL flattened curve and showed more small adipcoytes } \\
\text { had more adipocytes in the } 30-\text {, and } 40-\mathrm{mm} \text { categories }\end{array}$ \\
\hline OAT & $\mathrm{n} / \mathrm{a}$ & WL showed a rightward shift in distribution curve \\
\hline \multicolumn{3}{|l|}{ Cyclin A1 mRNA } \\
\hline EAT & no difference & $77 \mathrm{~d} 7 \mathrm{dWL}>77 \mathrm{~d}$ RUN \\
\hline PRAT & no difference & $77 \mathrm{~d} 7 \mathrm{dWL}>77 \mathrm{~d}$ RUN \\
\hline OAT & no difference & no difference \\
\hline SAT & no difference & 77d 7dWL > 77d RUN \\
\hline \multicolumn{3}{|c|}{$\begin{array}{l}\text { Comparisons of } 9 \text { outcome measures (body mass, lean mass, \% body fat, adipose tissue depot mass, } \\
\text { adipocyte diameter, total number of adipocytes in a depot, adipocyte size distribution, and Cyclin A1 mRNA } \\
\text { expression) between } 56 \mathrm{~d} \text { RUN and } 56 \mathrm{~d} 7 \mathrm{~d}-\mathrm{WL} \text { rats in the } 49-56 \text { day old group and between } 77 \mathrm{~d} \text { RUN and } \\
77 \mathrm{~d} \text { 7d-WL in the } 70-77 \text { day old group. EAT: epididymal adipose tissue, PRAT: perirenal adipose tissue, } \\
\text { OAT: omental adipose tissue, SAT: subcutaneous adipose tissue. }\end{array}$} \\
\hline
\end{tabular}




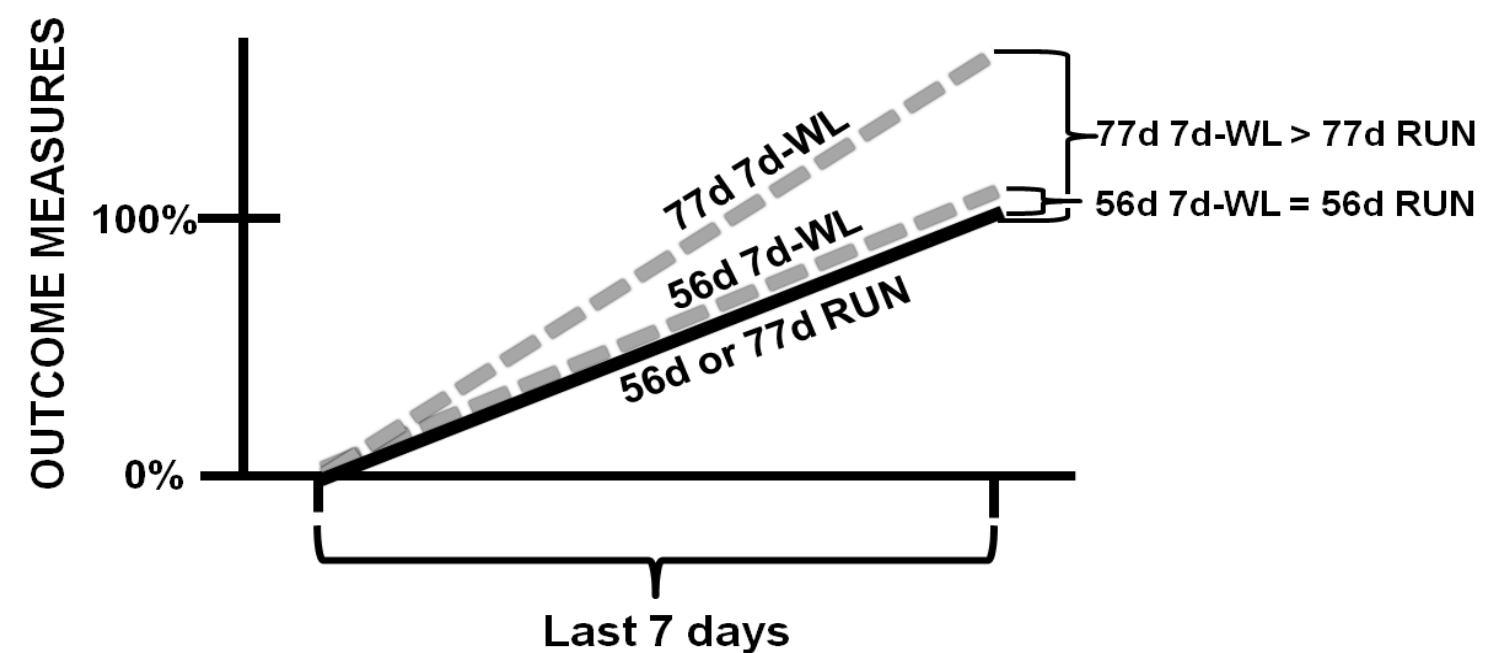

Figure 2.11. Summary Figure

Proposed two-phase construct illustrating differences observed between 49-56 day old rats and 70-77 day old rats in outcome measures examined for any co-clustering of directional changes or of no change. The $x-$ axis represents the final 7 days of the experiment, either 49- to 56-days of age or 70- to 77-days of age.. The $y$-axis represents the observed change in a given outcome measure over the final 7 days of the experiment. The black line represents both 56d RUN and 77d RUN groups and represents the entire change observed (100\%) over the last week. The two, gray dashed lines represent $56 d 7 d-W L$ and $77 d$ 7dWL groups. These lines represent changes observed in outcome measures relative to their respective RUN groups. In general, we observed almost no changes with WL in the 49-56 day old rats, while we noted many outcomes increase in the 70-77 day old rats.

Age of WL affects adipose tissue response

EAT in 49-56 day old RUN rats showed increased adipocyte size between 49 and 56 days of age even though they continued running, suggesting that these rats are in a hypertrophic growth phase. However the effect of WL appears to be 'lost' or 'masked' due to the natural expansion occurring with 7 days of age. In contrast, is seems clear that WL in 70-77 day old rats induces a hyperplasic response. There was a clear plateau in total adipocyte number in a depot between 70 and 77 days of age in both RUN and SED rats in all depots. WL induced an increase in total adipocyte number in EAT and PRAT. Age- 
matched run rats $(77 d \mathrm{RUN})$ did not exhibit this increase in total adipocyte number, thus the hyperplasic expansion in 70-77 day old rats is due to $\mathrm{WL}$, not age. These findings are novel and suggest that the age in which a decrease in activity occurs influences the expansion characteristics of visceral adipose tissue.

\section{WL alters adipocyte size distribution}

In the 49-56 day old rats, there is a clear rightward-shift with age. The 56d RUN and 56d SED curves are to the right of the 49d RUN and 49d SED curves, suggesting hypertrophic adipose tissue expansion. The WL curve shows a rightward-shift compared to $49 \mathrm{~d} \mathrm{RUN}$, but also shows a flattening and increased number of adipocytes in the $40 \mu \mathrm{m}$ category. In contrast, in the 70-77 day old rats, there is no longer a rightward-shift observed between the RUN groups or the SED groups in EAT and PRAT depots, suggesting that hypertrophic adipose expansion is no longer occurring. However, WL flattened the curve in both EAT and PRAT and showed more adipocytes in the 30 - and $40 \mu \mathrm{m}$ categories. The emergence of small adipocytes has been observed by others: weight-regain after calorie restricted weight loss in older rats $[98,101]$ and thiazolidinedione treatment in rodents [102], but this is the first time this has been measured in inactivity-induced fat mass increase in young rats. This data supports the supposition that 49-56 day old rats are still expanding adipose tissue by hypertrophy, while 70-77 day old rats are no longer expanding adipose tissue. In contrast to the other depots, the OMAT WL curve exhibited a rightward shift with no increase in smaller adipocytes, suggesting that OMAT adipocytes respond to WL by hypertrophy. Tchkonia et al. [103] reported that human omental pre- 
adipocytes have lower potential for replication and differentiation than subcutaneous or mesenteric pre-adipocytes in morbidly obese adult humans. While our rats were younger and not obese, this lower proliferation and differentiation potential in OMAT may explain the different response to $\mathrm{WL}$ in OMAT compared to EAT and PRAT.

There was no increase in inflammatory mRNA expression with WL

We measured three inflammatory markers, plus two hypoxic markers and found no increases in any of these with WL. Hypoxia markers were selected because hypoxia is implicated in early adipose tissue expansion and is a precursor to inflammation [104-106]. The lack of an inflammatory response may be due to hyperplasic adipose expansion, which is associated with a healthy adipose tissue adaptation [43]. Another explanation is that the adipose tissue expansion was not sufficient to evoke an inflammatory response. Many of the studies where an inflammatory response was observed used either longer periods of observation and/or a more severe induction of adiposity, like a high fat diet, $[19,107,108]$ or gene alteration $[109,110]$. While we can say that increases in adipose tissue mass seen with a sudden decrease in activity are not accompanied by a pro-inflammatory phenotype in these young rats, we are not certain of the effects of decreased activity as these animals age. 
WL cyclin A1 mRNA expression equaled that of SED and was greater than RUN

We found new evidence that suggests cell proliferation is increased EAT, PRAT and SAT in WL rats. Cyclin A1 is a marker of proliferation, as its protein functions to promote cell proliferation and survival by its requirement for $\mathrm{S}$ phase and passage through G2 [111, 112]. Cyclin A1 mRNA was greater in 77d 7d-WL than in 77d RUN group for 3 adipose tissue depots (EAT, PRAT, and SQAT), without similar differences between 56d RUN and 56d 7d-WL (Fig. 2.10). The emergence of a higher proportion of small adipocytes $(<30 \mu \mathrm{m})$ in EAT and PRAT of 70-77 day old rats after one week of WL combined with the increase in cyclin A1 mRNA expression supports the notion of an inactivity-induced proliferation of preadipocytes and/or differentiation of preadipocytes present in $7 d-W L$ depots. We previously reported the occurrence of hyperplasia in the EPI depot with 7d-WL [60], and others have found adipocyte proliferation to occur with weight regain after calorie restriction in rats, $[98,101,113]$ and in weightreduced humans [114]. Thus, we suggest the novel idea that physical activity acts as a 'brake' on adipose tissue expansion via hyperplasia in 70-77 day old rats, and once that 'brake' is removed (i.e., WL), adipose tissue expands primarily by hyperplasia. The preferential increase in cyclin A1 prior to other mRNA changes in only the 70-77 day old WL group supports formation of the following hypothesis: an upstream 'activity'-dependent signal to the cell cycle, and not a pro-inflammatory adipokine, is an early signal to increase hyperplasia. 
Adipose tissue depots responded differently to $W L$

The above data clearly shows that the three adipose depots respond differently to $\mathrm{WL}$ in 70-77 day old rats. We speculate that differential responses among intra-abdominal fat depots are most likely to be due to intrinsic differences in gene programming in various intra-abdominal fat depots and to age, rather than to the stage of sexual maturity, as the latter is a commonality to the various intra-abdominal fat depots. In support of our notion, it has previously been reported that the inherent proliferative capacity of adipose depots vary: SAT > PRAT > EAT > mesenteric AT [115], as well as metabolic differences between depots [35].

A differential response in adipose tissue depots may have clinical implications given that new, smaller adipocytes have greater capacity to preferentially accumulate fat compared to larger adipocytes, and thereby buffer the onset of the metabolic syndrome. In addition, smaller adipocytes are less sensitive to anti-lipolytic effects of insulin [116], they exhibit lower rates of basal and catecholamine-stimulated lipolysis $[117,118]$, and they are more protective against metabolic disease as compared to very large adipocytes [54]. The emergence of small adipocytes has been observed by others: weight-regain after calorie restricted weight loss in older rats $[98,101]$ and thiazolidinedione treatment in rodents [102]. Therefore, we speculate that the failure of omental adipocytes to proliferate during WL in 70-77 day old rats could play a contributing role to omental adipose tissue being an unhealthier adipose tissue depot. 
Resolution of previous data discrepancies.

We previously reported that during WL, adipocyte hypertrophy predominated during increased EAT mass in Fischer 344 Brown Norway rats aged 42-49 days of age [61], but that hyperplasia was dominant in rats at the ages of 70-77 days old [60]. Our current results in Wistar rats suggest a resolution to our previous two conflicting sets of data in that both of our previous papers are correct. The juvenile age of growth when WL takes place affects adipose tissue response. WL effects are essentially masked in younger rats, but we see increased cell size from 49 to 56 days regardless if the rats continue running (49d RUN $<56 d R U N)$ or undergo WL (49d RUN $<56 d$ WL), suggesting that hypertrophy is occurring regardless of physical activity levels. However in 70-77 day old rats, WL increased adipocyte number, suggesting a hyperplasic response.

Disconnect between decreased food intake and increased adiposity

The rapid decreased appetite within the first 3 days of WL merits comment (Fig. 2.5B), as this is not the first time we have reported this decrease in WL experiments in lean rats [60]. The common view is that reduced caloric intake would lower fat storage. Instead, we report that food intake reduction was associated with gain in body fat when there is decreased physical activity, implying decreased energy expenditure. Thus, a remarkable disconnect exists with common dogma that a reduction in caloric intake reduces body fat. Physical 
activity must be factored into this equation, but the caloric cost of reduced physical activity can only be assumed, making this a limitation to this interpretation. Interestingly, we observed an initial increase in feed efficiency on the first day of WL that was immediately followed by a lower value on the $2^{\text {nd }}$ through $6^{\text {th }}$ WL day. This phenomenon has been observed in other weight gain studies, [119] and suggests a lower energetic cost of weight gain during the initial days of WL. A new question is thus raised as to whether regulatory mechanisms exist to rapidly lower food intake and alter feed efficiency when physical activity is decreased.

Regulatory mechanisms responsible for increased fat gain with both decreased caloric intakes and expenditures in lean rats have not been intensely examined. One possible consideration might be related to juvenile rats with chronic voluntary running having lower \% body fat, which we speculate would lower blood leptin and increase leptin sensitivity to inhibit appetite once running stopped. Our speculation is based upon a report by Patterson et al. [120], in which 3 wks of voluntary running was associated with increased sensitivity to the anorectic effects of leptin without changes in plasma leptin. We did not measure leptin in these rats, so this question remains to be answered. The immediate loss of voluntary running also initiated a spike in food efficiency on the first day of WL, which returned down to its pre-WL level on the second WL day. This phenomenon has been observed in other weight gain studies [119], and suggests a lower energetic cost of weight gain during the initial days of WL. MacLean et al. [119] have hypothesized that this initial reduced cost of weight 
gain in re-feeding after caloric restriction is due to preferential fuel utilization shifts towards carbohydrate oxidation and lipid storage in adipose tissue [121]. Hence, it would be of future interest to delineate mechanisms by which WL may be affecting feed efficiency. Likewise, it would be worthwhile to research how energy expenditure prior to and after WL affects appetite altogether.

Notwithstanding, our data supports Blundell et al. [122] who suggest that energy expenditure can be added to the list of factors regulating energy intake and appetite control, and continues to provide compelling evidence suggesting that a swift increase in adiposity occurs during the onset of inactivity despite decreases in food intake.

\section{Limitations of the study}

There are other iterations of WL that could be performed to further understand visceral adipose expansion with WL. For example, to determine if the factor inducing hyperplasic visceral expansion is age or duration of running, we could employ a group that gains access to a voluntary running wheel for three weeks from 49 to 70 days of age, and is wheel locked from 70 to 77 days of age. This group would be age matched with the 70-77 day old rats that had six weeks access to a voluntary running wheel. If the group with access for only three weeks showed hyperplasic visceral expansion, then we gain more support to our conclusion. However, if the group with access for only three weeks showed hypertrophic visceral expansion, then the duration of voluntary wheel running must be considered as a factor in the type of visceral adipose expansion. 
In conclusion, a rodent model for childhood developmental changes in adipose tissue was investigated after one week of greatly reduced daily physical activity (7d-WL). We found that: 1) more robust changes with WL were observed in the 70-77 day old rats than in the 49-56 day old rats. Compared to $77 d$ RUN rats, $77 d 7 d-W L$ rats exhibited greater rates of gain of fat mass and $\%$ body fat, increased adipocyte number, higher percentage of small adipocytes, and greater cyclin A1 mRNA expression in epididymal and perirenal adipose tissue. In contrast, 49-56 day old rats had no change in most of the same characteristics. 2) In both age groups, WL did not increase inflammatory mRNA expression. 3) There was a disconnect between decreased food intake and increased adiposity, suggesting that reduced caloric intake does not reduce body fat when energy expenditure is reduced simultaneously.

From observations in this study, we now present a new hypothesis in Chapter 3 that visceral adipose tissue, specifically EAT and PRAT, is expanding by hyperplasia in this rat model due to an increase in cell cycle, proliferation, and differentiation induced by WL.

\section{Perspective and Significance}

In the context of the current childhood obesity epidemic, there is an urgent need to understand underlying mechanisms of adipose tissue growth and investigate obesity-prevention strategies. Since no single tissue or factor can be fully responsible for weight regain, the wheel lock model provides a unique environment to study how a sudden cessation of daily physical activity affects 
some characteristics of adipose tissue in pre-pubertal rats. One alternating period of physical activity and inactivity had consequential effects on adipose cell number in the EAT and PRAT depots of 70-77 day old rats. As human childhood usually consists of multiple intervals of activity-inactivity, the pre-clinical consequences of repeated periods of activity with inactivity should be further investigated with invasive approaches using said animal models. 


\section{CHAPTER 3}

\section{STUDY 2:}

GLUCOCORTICOID RECEPTOR BLOCKER RU486 ATTENUATES FAT GAINS INDUCED BY A SUDDEN DECREASE IN PHYSICAL ACTIVITY, BUT BY DIFFERENT PATHWAYS THAN PHYSICAL ACTIVITY 


\section{ABSTRACT}

Voluntary running and glucocorticoids block adipose tissue expansion. Young rats that undergo a sudden decrease in daily physical activity [wheel lock $(\mathrm{WL})]$ at 70-77 days of age exhibit visceral adipose tissue expansion by hyperplasia. We questioned whether blocking glucocorticoid action would attenuate visceral adipose tissue expansion during $\mathrm{WL}$ as running attenuates visceral adipose expansion. We tested the hypothesis that similar changes in perirenal adipose tissue (PRAT) transcript expression involved in cell cycle, proliferation, and differentiation would occur in rats that have access to a voluntary running wheel (RUN) and rats that undergo wheel lock plus administration of a glucocorticoid receptor block (WL+GR BLOCK) when compared to WL rats. RNA-sequencing (RNA-seq) was performed on PRAT on 173 transcripts specific to cell cycle, proliferation, and differentiation. RUN and WL+GR BLOCK showed no differences in change in fat mass or change in $\%$ body fat from 70-77 days of age, or differences in PRAT mass at 77 days. RNAseq analysis of the 173 transcripts showed 71 differentially expressed transcripts (DETs) between RUN and WL, but only 20 DETs between WL+GR BLOCK and WL. Uniquely up- and down-regulated transcripts were identified. We conclude that RUN and WL+GC BLOCK did not affect cell cycle, proliferation, and differentiation in the same manner. The data provide novel insight that two different molecular mechanisms block adipose expansion, and stresses the importance that a drug does not fully mimic physical activity. 


\section{INTRODUCTION}

The previous chapter highlighted the growing crisis in public health as children become less physically active, coinciding with increased adiposity and physical activity during adolescent years [123]. Wheel lock (WL) induces a hyperplasic adiposity rebound in epididymal adipose tissue (EAT) and perirenal adipose tissue (PRAT) depots. The mechanism behind this phenomenon is still unknown. The explanation may be as simple as maintaining a high caloric intake (positive caloric balance) with a decrease in activity, but the previous chapter showed that rats compensate by decreasing food intake within three days after WL. Or the explanation may be more complicated.

Glucocorticoids (GCs) are of particular interest due to the role they play in adipose tissue homeostasis and that glucocorticoid receptor blockers (GR BLOCK) have been effectively used to stop fat deposition [88]. Given the seriousness of the childhood obesity problem, mechanisms by which we can attenuate fat gains with a sudden decrease in activity may be elucidated by a comparison of GR BLOCK and WL. The primary aim of this study was to determine whether a GR block attenuates fat gains seen with WL in a similar manner as running does.

GCs (cortisol in humans and corticosterone in rodents) are steroid hormones released from the adrenal glands that are important in times of physiological or psychological stress [124] and have catabolic effects on lipid and protein metabolism with anabolic effects on glycogen storage [76, 125]. GCs bind the glucocorcoidoid receptor (GR), which then acts as a transactivator or 
transrepressor. While the role of GCs in liver and skeletal muscle is relatively clearly defined, the role of GCs in adipose tissue is controversial. GCs are most commonly believed to be lipolytic [76] and increase the amount of energy substrates in the blood during energy deprivation [124], but GCs also promote preadipocyte differentiation and adipogenesis [77]. GC treatment in children for conditions such as asthma and rheumatic diseases, inhibit weight-loss efforts and aggravate weight gain [85-87]. GCs are regulated by the enzymes 11-beta hydroxysteroid dehydrogenase type 1 (11ßHSD-1, highly expressed in adipose tissue) and type 2 (11ßHSD-2, highly expressed in the kidney). $11 \beta \mathrm{HSD}-1$ functions predominately to activate glucocorticoids, while $11 \beta \mathrm{HSD}-2$ primarily inactivates glucocorticoids [126]. Interestingly, in animal models when 11ßHSD1 is reduced (thus decreasing tissue exposure to active GC), adipose tissue is redistributed towards subcutaneous fat, and animals show improved metabolic and blood lipid profiles [127-129].

GRs are more highly expressed in visceral fat (VAT) than other fat depots [78], and GCs impair insulin sensitivity in adipocytes and promote free fatty acid release through hormone sensitive lipase [130]. Further, increased exposure to GCs in insulin-sensitive tissues is associated with lipid accumulation and metabolic complications [79-83]. Interestingly, GCs affect different fat depots differently; they increase lipolysis in peripheral fat depots, like subcutaneous adipose tissue (SAT), but they promote pre-adipocyte differentiation and adipocyte hypertrophy in VAT [131], so there is something unique to VAT regarding $\mathrm{GC}$ action. 
Protection against weight gain, decreased body fat, and a healthier adipose tissue phenotype are among the long list of benefits that physical activity has on physiology $[66,67]$. Chronic physical activity like endurance training also makes tissue more sensitive to GCs, and increases lipolysis in adipose tissue [73]. While this provides a valuable energy source for exercising animals, it could be detrimental for sedentary animals though increased plasma lipids and adipose tissue redistribution towards the viscera [74, 75].

Mifepristone, or RU486, is a synthetic steroid that acts as an antagonist at progesterone and glucocorticoid receptors [132]. RU486 is commonly used for termination of pregnancy and emergency contraception, but is also used in treatment of burns, and Cushing's syndrome [132]. In addition to these roles RU486 has a potential role in obesity-related disorders like diabetes and hypertension by indirectly affecting adipose tissue through the anti-glucocorticoid effect $[88,133-135]$.

Few transcriptome-scale studies have been performed on adipose tissue and none have been performed on adipose tissue in the context of WL or GR block and physical activity. RNA sequencing (RNA-seq) is a highly reproducible, sensitive technique that allows us to observe an entire transcriptome [136]. In this study, we performed RNA-seq to investigate quantitative and qualitative transcriptome signatures of PRAT from rats that had uninterrupted access to voluntary running wheels (RUN), WL, and WL plus GR block (WL+GR BLOCK).

Prior to RNA-seq analysis, we observed that WL+GR BLOCK rats maintained similar levels of adiposity as $R U N$ rats. Specifically, $R U N=W L+G R$ 
BLOCK $<W L$ occurred in the following measurements: change in body fat $\%$, change in fat mass, PRAT depot mass, and Cyclin A1 mRNA expression, and RUN=WL+BLOCK occurred in PRAT adipocyte diameter. The perirenal fat depot was selected for RNA-seq analysis because it is representative of visceral fat, is a major adipose target of GC action [137], and exhibited dynamic changes in a previous study (Chapter 2).

Based on this preliminary data and data from other studies [[60] and Chapter 2], my overall hypothesis for this study is that, when compared to WL, RUN and WL+GR BLOCK would show similar changes in PRAT transcript expression involved in cell cycle, proliferation, and differentiation.

\section{METHODS}

Animals and Experimental design

The University of Missouri Animal Care and Use Committee approved experimental protocols. Twenty-four male, Wistar rats were randomly selected from a population of Wistar rats bred for high running distances in our laboratory. The rats were weaned at 21 days of age and housed as a litter in a single cage. At 28 days of age, rats were relocated to a cage equipped with a voluntary running wheel and a Sigma Sport BC 800 bicycle computer (Cherry Creek Cyclery, Foster Falls, VA) where they were individually housed for the remainder of the experiment (Fig. 3.1). Rats were randomly selected to be in one of four groups for the (RUN, WL, WL+GR BLOCK, and WL+VEHICLE, $n=6$ per group). 
RUN rats had access to a voluntary running wheel from 28 days of age through 77 days of age. WL, WL+GR BLOCK and WL+VEHICLE rats had access to a voluntary running wheel from 28 days of age through 70 days of age, when the wheel was locked for the last 7 days. In addition to $\mathrm{WL}, \mathrm{WL}+\mathrm{GR}$ BLOCK and WL+VEHICLE rats received an injection of either DMSO plus $20 \mathrm{mg} / \mathrm{kg} \mathrm{RU} 486$ (WL+GR BLOCK), which was diluted to a volume of $\sim 0.5 \mathrm{ml}$ per injection, or $0.5 \mathrm{ml}$ DMSO (WL+VEHICLE) once daily at $17.00 \mathrm{~h}$ (2 hrs prior to the dark, physically active 12-hr period). This dosage was chosen based on previous RU486 rat studies [138]. The time was chosen based on the circadian rhythm of cortisone; in rats, the level of corticosterone is highest just before waking [139] (rats are nocturnal and wake at 19.00h).

All rats were maintained on a 12-h light (07.00h-19.00h), 12-h dark (19.00$7.00 \mathrm{~h})$ cycle and housed in the same temperature-controlled animal room $\left(21^{\circ} \mathrm{C}\right)$. All rats had ad labium access to water and standard rodent chow (Formulab 5008, Purina Mills, St. Louis, MO, USA). Running activity was recorded daily and was determined by time and distance ran during the previous 24 hours. Animal cages were exchanged with clean cages every 7 days at which time weekly food intake and body weight were recorded. Rats from the RUN and WL groups are a randomly selected subset of rats that were used for the experiment in Chapter 2. Rats in the WL+GR BLOCK and WL+VEHICLE groups are unique to this experiment. Rats were family-matched as best as possible; when a litter of at least 4 males was available, one rat was assigned to each group. 


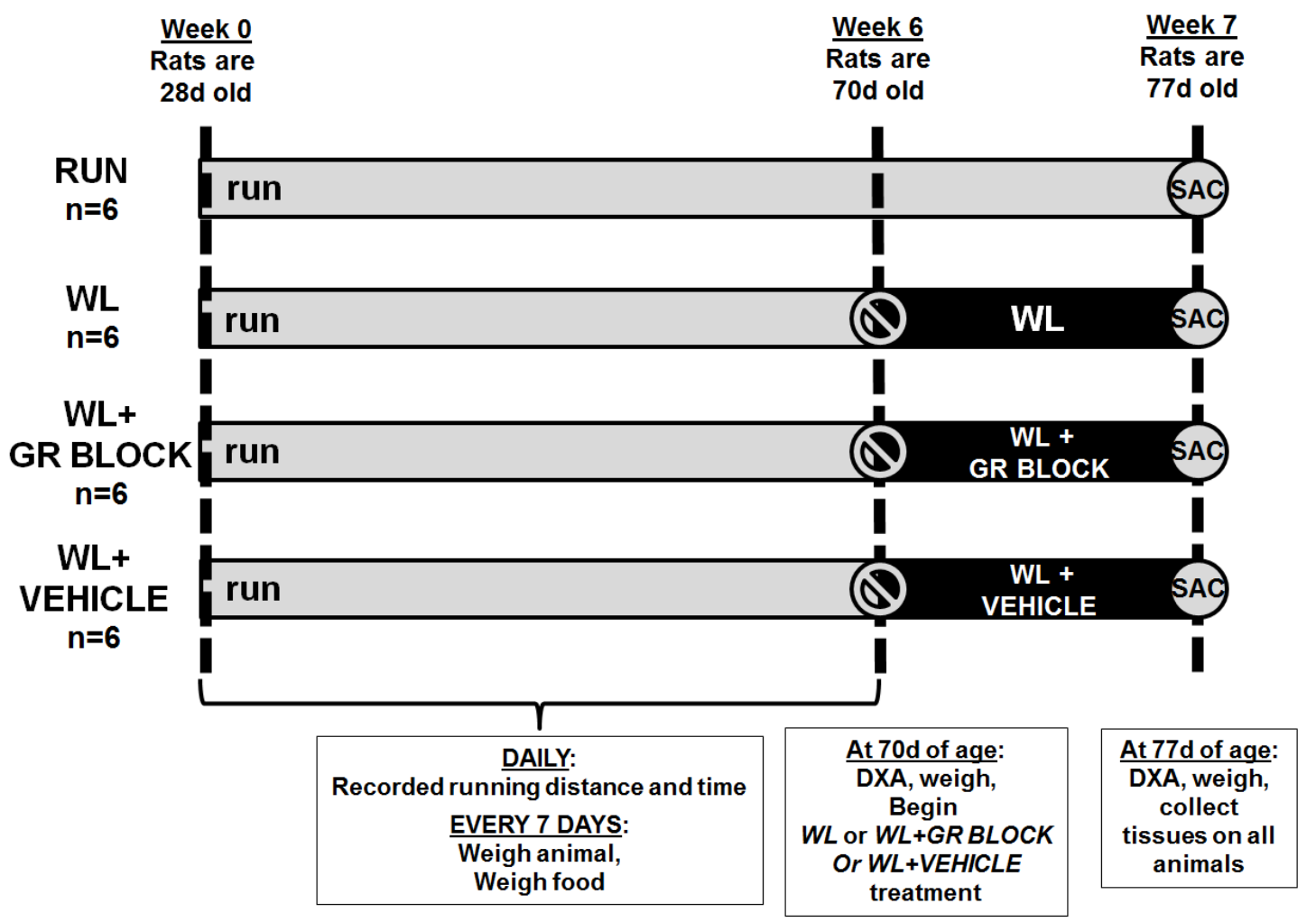

Figure 3.1. Experimental Design

Experimental groups are named by the treatment. All rats began running at the same age ( 28 days of age), underwent treatment at the same age (70 days of age), and were sacrificed at the same age (77 days of age). Light gray bars represent access to a voluntary running wheel, while black bars represent wheel-lock, where the rats were housed in the same cage as previously, but the running wheel did not turn (was locked). RUN rats had access to a voluntary running wheel for 7 weeks. WL rats had access to a voluntary running wheel for 6 weeks followed by wheel-lock for 1 week. WL+GR BLOCK rats had access to a voluntary running wheel for 6 weeks followed by wheel-lock plus a $\sim 0.5 \mathrm{ml}$ (adjusted to accommodate the correct dosage) IP injection of RU486 in DMSO daily (20 mg/kg). WL+VEHICLE rats had access to a voluntary running wheel for 6 weeks followed by wheel-lock plus $0.5 \mathrm{ml}$ IP injection of DMSO daily. The day that rats underwent treatment (70 days of age), they were DXA scanned and weighted. The day of sacrifice (77 days of age), the rats were DXA scanned, weighed, blood was drawn for plasma analysis, and fat pads were removed for phenotyping and RNA analysis.

\section{Body composition analysis using DXA}

At 70 and 77 days of age, body composition was measured between

10.00h and 12.00h using a dual-energy X-ray absorptiometry (DXA) machine calibrated for rats (QDR 4500A, Hologic, Inc. Bedford, MA). The DXA scan at 70 days was performed after anesthetization by isofluorine. At 77 days of age, the 
DXA scan was performed after $\mathrm{CO}_{2}$ asphyxiation and immediately prior to tissue removal.

\section{Animal Sacrifice and tissue collection}

Rats were terminated by $\mathrm{CO}_{2}$ asphyxiation between $10.00-12.00 \mathrm{~h}$, weighed, and DXA scanned as described previously. Four adipose pads were removed: omental adipose tissue (OMAT), epididymal adipose tissue (EAT), perirenal adipose tissue (PRAT), and subcutaneous adipose tissue (SAT). OMAT, EAT, and PRAT depots were completely removed and weighed, while only a portion of umbilical/groin SAT was collected. A sample of OMAT, EAT, and PRAT was used for adipocyte sizing (described below), and a sample of each of the four fat depots was snap-frozen in liquid nitrogen and stored at $-80^{\circ} \mathrm{C}$ for analysis. Blood was drawn via a cardiac puncture (needle and syringe) for plasma analysis.

\section{Adipocyte sizing and number}

On the day of sacrifice, three $60-80 \mathrm{mg}$ fresh adipose tissue fragments from OMAT, EAT, and PRAT were weighed and placed in a glass scintillation tube with $3 \mathrm{ml}$ Krebs-Ringer-HEPES buffer (KRBH; $130 \mathrm{mM} \mathrm{NaCl}, 4.7 \mathrm{mM} \mathrm{KCl}$, $1.2 \mathrm{mM} \mathrm{KH}_{2} \mathrm{PO}_{4}, 10 \mathrm{mM}$ HEPES, $1 \mathrm{mM} \mathrm{CaCL} 2,1.2 \mathrm{mM} \mathrm{MgSO}_{4}, 0.25 \mathrm{mg} / \mathrm{ml}$ free fatty acid-free $\mathrm{BSA}, \mathrm{pH}=7.4$ ). Fragments were minced into $\sim 1 \mathrm{~mm}^{3}$ pieces, incubated with 0.920 Wunsch units of Liberase Blendzyme (Roche) in a $37^{\circ} \mathrm{C}$ incubator at $100 \mathrm{rpm}$ for 1 hour, transferred through a sterile $200 \mu \mathrm{m}$ nylon mesh 
(Sefar America, Kansas City, MO) into a $15 \mathrm{ml}$ conical, and centrifuged at 100 rpm for 1 minute to allow adipocytes to loosely pack at the top of the liquid. The bottom layer of liquid was removed using a syringe and needle and the adipocytes were washed with $\sim 5 \mathrm{ml} \mathrm{KRBH}$ and resuspended in $5 \mathrm{ml} \mathrm{KRBH}$ for every $1 \mathrm{ml}$ of loosely packed adipocytes. A random sample of adipocytes was viewed with a Nikon Eclipse E600 microscope and photographed by an Olympus DP72 camera. The diameter of $>300$ adipocytes was measured using DP2-BSW v2.1 software. The mean diameter and size distribution were calculated for each adipocyte depot.

The number of adipocytes per depot was estimated from the following calculation: the average cell mass was calculated by: average cell size $(\mathrm{pl}) \mathrm{x}$ 0.915 (ng lipid $/ 1 \mathrm{pl}$ ) $\times \mathrm{g} / 10^{9} \mathrm{ng}$; the number of cells per depot was calculated as: mass of adipose depot (g) / average mass of adipocyte (g). Reliability of this method is described in detail [119] and has been used by others [101].

\section{Corticosterone plasma assay}

Blood plasma was collected by withdrawing blood with a heart stick (needle and syringe) and placing the blood in a plasma separating tube. After 30 minutes at room temperature, the blood was centrifuged for 10 minutes at $1300 \mathrm{x}$ $\mathrm{g}$, and the plasma fraction separated to the top layer. Plasma was pipetted from the tube and placed in a sterile $1.5 \mathrm{ml}$ tube. Corticosterone levels were measured from commercially available corticosterone ELISA kit (Abcam, Cambridge, MA) according to the manufacturer's instructions. 
RNA isolation for RNA sequencing

The perirenal fat depot was selected for RNA-seq analysis because it is representative of visceral fat, is a major adipose target of GC action [137], and exhibited dynamic increases in size in a previous study (Chapter 2).

Approximately $100 \mathrm{mg}$ of frozen PRAT was homogenized in $1 \mathrm{ml}$ RNA lysis buffer (Tri Reagent, Sigma, St. Louis, MO) and a stainless steel bead using a high-speed shaking device at $30 \mathrm{~Hz}$ for 1 min (Tissuelyser LT, Qiagen, Valencia, CA). RNA was isolated using manufacturer's instructions (Tri Reagent, Sigma, St. Louis, MO). RNA integrity was checked using BioAnalyzer 2100 (Agilent Technologies Inc., Santa Clara, CA) automated electrophoresis system (Bio-Rad, Hercules, CA) prior to cDNA library construction at the University of Missouri DNA Core.

The cDNA library was constructed at the University of Missouri DNA Core using manufacturer's protocol with reagents from Illumina's TruSeq RNA sample preparation kit v2 (Illumina, San Diego, CA). Briefly, 1) the poly-A containing mRNA was purified from total RNA. 2) RNA was fragmented. 3) cDNA was generated from this fragmented RNA. 4) sample identifier adapters were ligated to the ends. 5) the final constructs were evaluated using the BioAnalyzer 2100 automated electrophoresis system. 6) these constructs were quantified with the Qubit fluorometer (Invitrogen, Carlsbad, CA) using the quant-iT HS dsDNA reagent kit (Invitrogen). 7) this was diluted for HiSeq2000 (Illumina, San Diego, CA) using Illumina's standard sequencing protocol. 


\section{RNA sequencing}

RNA-seq procedures were performed at the University of Missouri's DNA Core and performed as described elsewhere [140]. Briefly, 1) samples were loaded into a flowcell where each olgio was replicated. 2) flowcells were placed in the sequencer and fluorescently labeled bases were attached to each complementary base. 3) 50 base pair (bp) reads were recorded with the Illumina Genome Analyzer (Illumina, San Diego, CA). 4) reads were trimmed to confirm adapter sequence removal. 5) reads were tiled to a custom reference using NextGEN3 v1.92 software (SoftGenetics, State College, PA). 6) RNA-seq sequences for each biological sample were trimmed of adapter and redundant sequences. 7) these RNA-seq sequences were aligned to a custom database consisting of rat and human homologous sequences using NexGen v2.2 (SoftGenetics, State College, PA). Reads per kilobase million (RPKM) values were assigned to each transcript. RPKM is a value that quantifies gene expression from RNA sequencing data that normalizes for the number of sequencing reads and total read length [141] and is the value that is used to compare transcript expression between groups. 

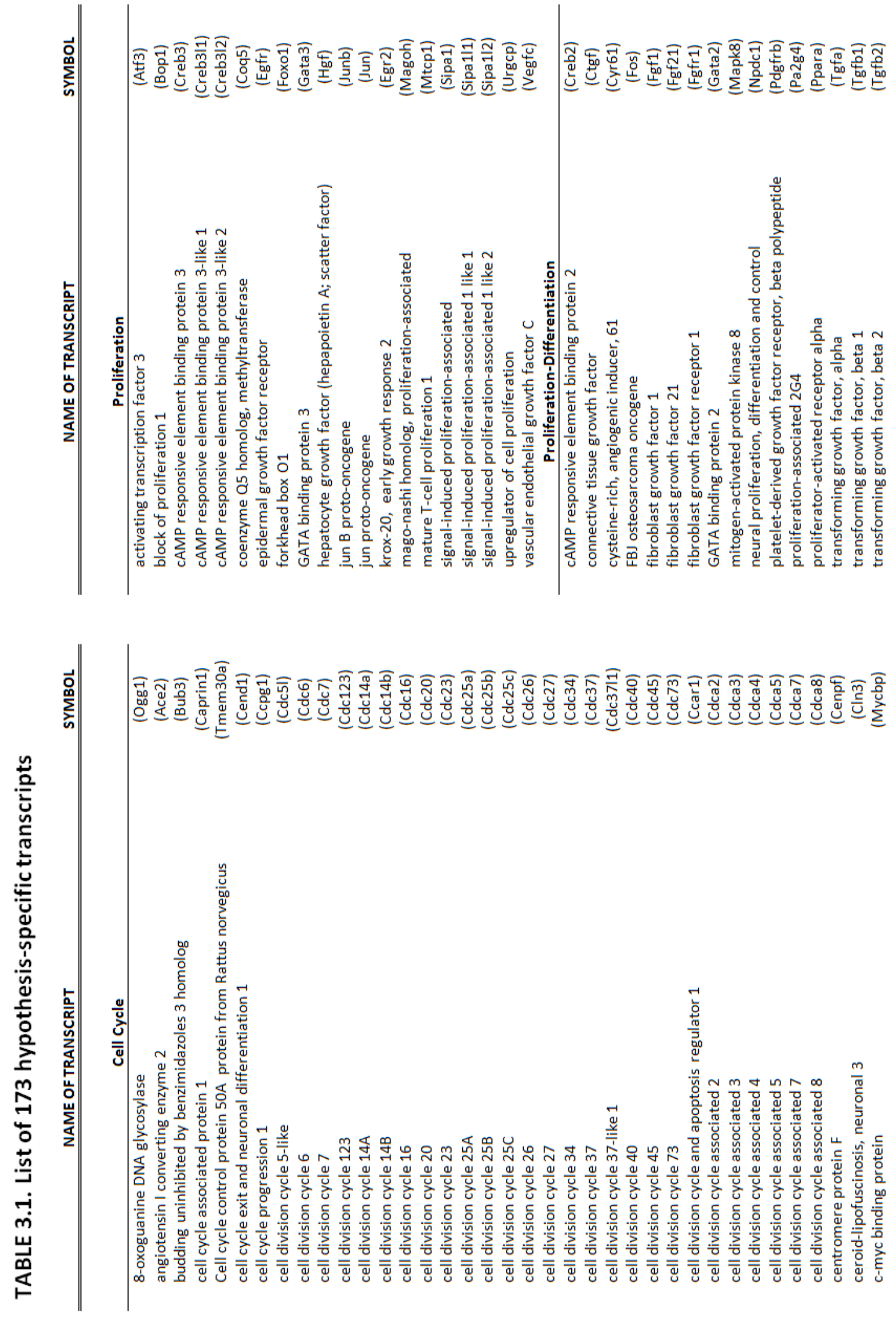


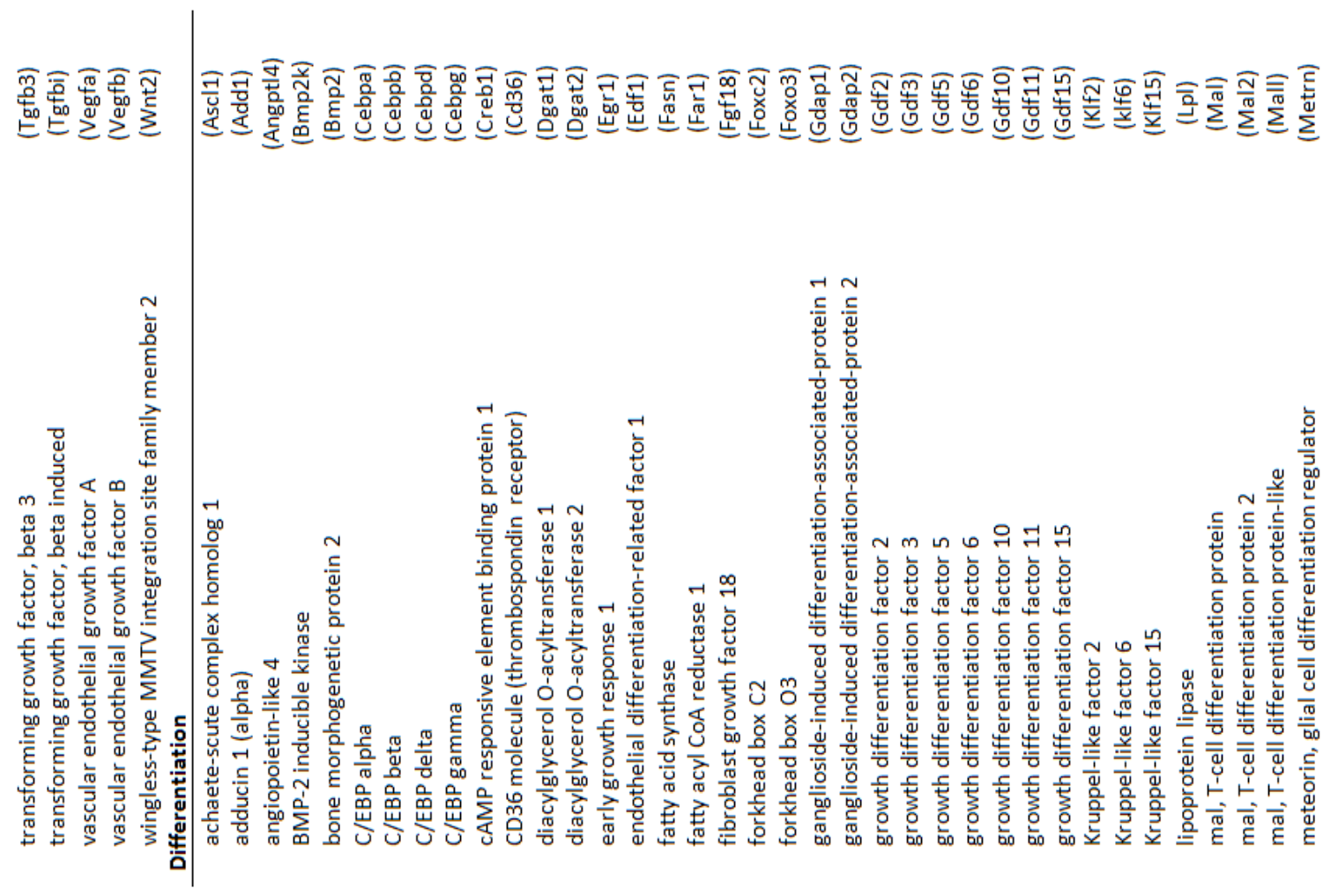

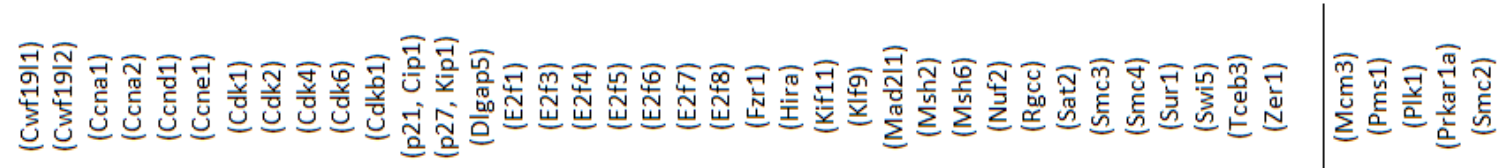

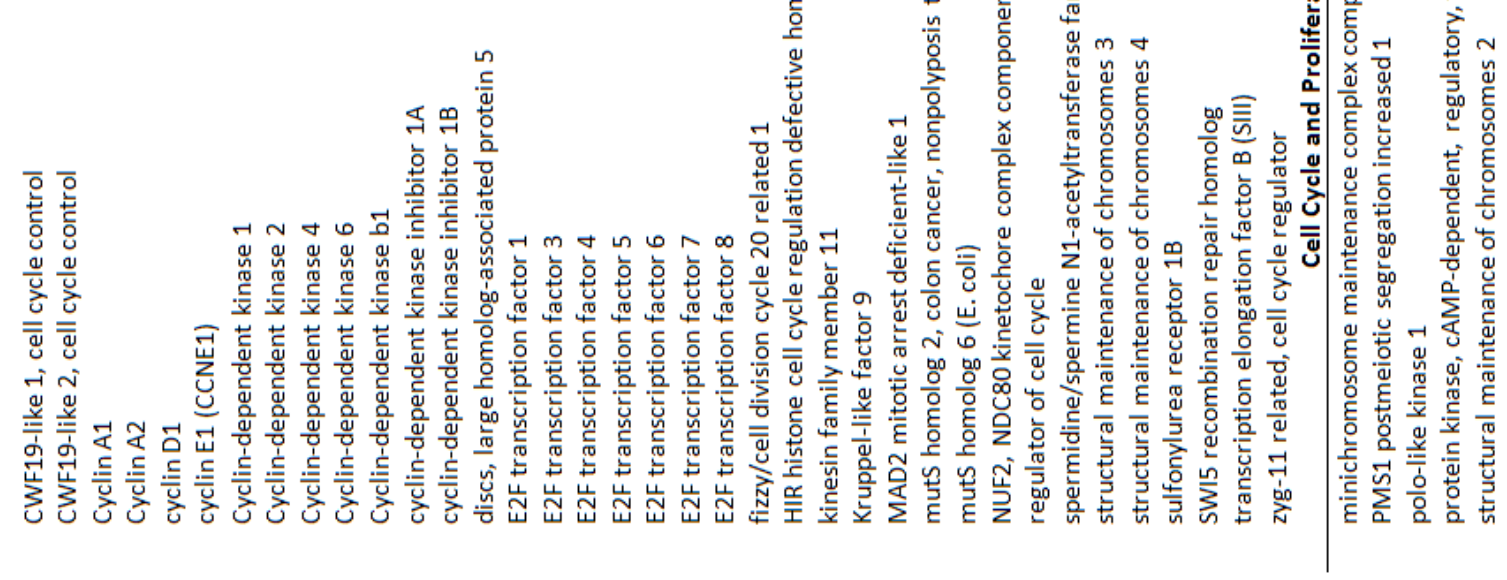



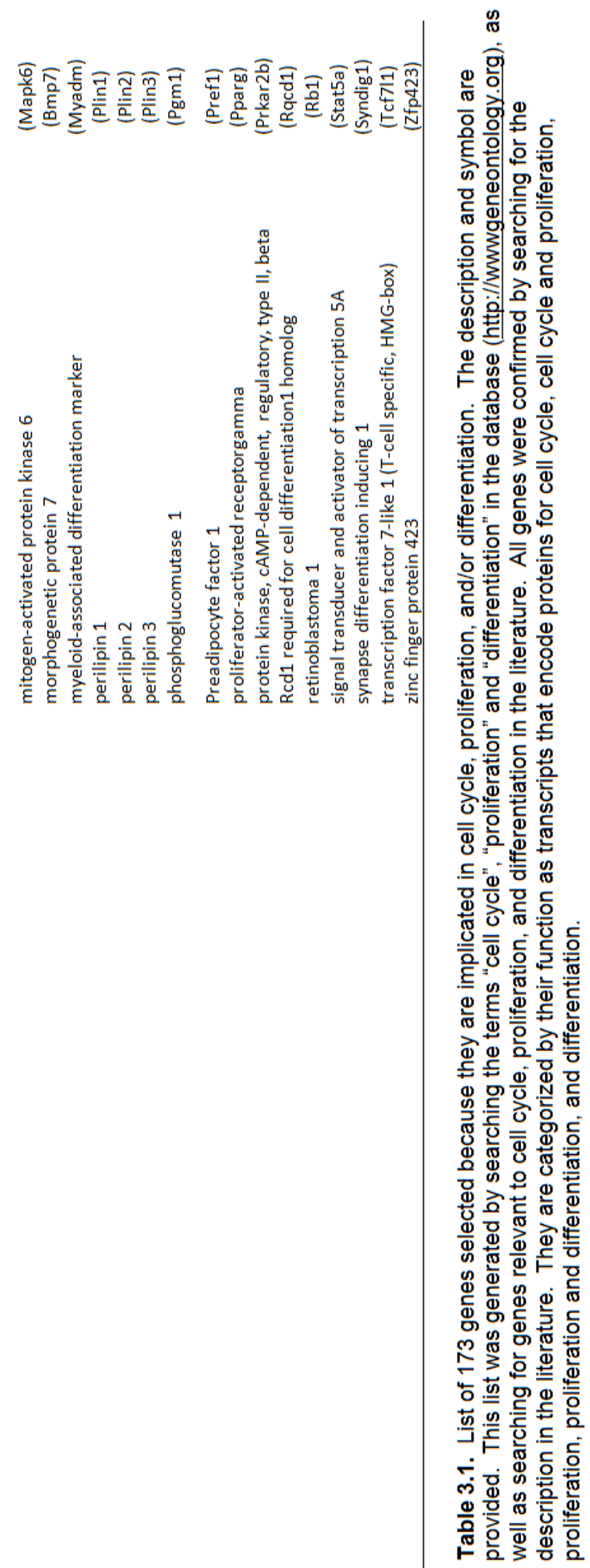
RNA Sequencing filtering process

As of now, there is no common standard for RNA-seq analysis, so the current RNA-seq literature was examined and a method was devised for a filtering process that incorporated aspects of current filtering procedures into the dataset. All analytical procedures were performed with Microsoft Excel v2007 (Redmond, WA). To directly address the hypothesis that when compared to WL, RUN and WL+GR BLOCK would show similar changes in PRAT transcript expression involved in cell cycle, proliferation, and differentiation, a hypothesisspecific filtering process was employed. The filtering process is described in detail and shown in Figure 3.5 in the results section. Briefly, the filtering process is as follows: 1) a list of 173 transcripts specific to the hypothesis was generated by imputing the term "cell cycle, "'cell cycle', species 'R. norvegicus", “'proliferation', species 'R. norvegicus'”, and “'differentiation', species 'R. norvegicus"' into Gene Ontology Consortium database (http://wwwgeneontology.org), as well as searching for genes relevant to cell cycle, proliferation, and differentiation in the literature (Table 3.1), 2) three pairwise comparisons were performed: [RUN versus WL], [WL+GR BLOCK versus $\mathrm{WL}$ ], and [RUN versus $B L O C K]$. [RUN versus $W L]$ and [WL+GR BLOCK versus $W L]$ were used for the initial filtering process, while [RUN versus $W L+G R$ BLOCK] used as criteria for the final filter) [note: Since the hypothesis was that RUN and WL+GR BLOCK would show similar changes in PRAT transcript expression, I chose to make WL the control and measure how each of the two 'treatments' (RUN and WL+BR BLOCK) compared to WL.], 3) FILTER \#1 - 
identify significant transcripts: Transcripts were considered significantly different if $p<0.05$, and significant transcripts for the [RUN versus $W L]$ and $[W L+G R$ BLOCK versus WL] were identified as up-regulated or down-regulated, 4) FILTER \#2 - identify transcripts that were "unique to RUN" and "unique to WL+GR BLOCK": transcripts that were identified as commonly up- and downregulated between [RUN vs. WL] and [WL+GR BLOCK vs. WL] were omitted, 5) from the remaining transcripts, unique transcripts to RUN and to WL+GR BLOCK were determined by: a) $R P K M \geq 1$ and $R U N \neq W L+G R$ BLOCK. Filters were chosen based on other RNA-seq literature [136].

Ingenuity Pathway Analysis (IPA; Ingenuity Systems, Inc., Redwood, CA) was used to identify pathways uniquely up-and down-regulated in RUN. This was not performed on BLOCK due to the small number of transcripts that were deemed 'unique.'

Additionally, to observe the entire PRAT transcriptome without limiting the analysis to cell cycle, proliferation, and differentiation transcripts, RNA-seq analysis was performed using a second, different filtering process. Our filtering process for the entire data set is described next in detail and shown in Figure 3.7 of the results section. Briefly, the filtering process is as follows: 1) the unknown transcripts were filtered out of the entire list of transcripts. 2) the transcripts from all 6 rats in a given group were compared and transcripts that were not present in all six rats were omitted. 3) two pair-wise comparisons were performed for [RUN vs. WL] and for [WL+GR BLOCK vs. WL]. 4) transcripts that were commonly expressed in both groups were analyzed and considered significant if they 
passed three filters: a) $p<0.005$, b) fold change $\geq 1.5$, and c) average RPKM $\geq$ 1.0. 5) transcripts that were unique to one group but not the other were analyzed separately and considered significant if they passed the same three filters as previously described. Filters were chosen based on other RNA-seq literature [142-144].

\section{Statistical analysis}

Outcome measures for between-group comparisons and within-group comparisons (body mass, lean mass, fat mass, and body fat \%) were analyzed with a two-way analysis of variance (group $x$ time). Significant differences are noted with lower-case letters in tables and graphs. Delta measurements (change in body weight, lean body mass, fat mass and \% body fat) and tissue measurements (depot mass, adipocyte diameter, and total adipocyte number) were analyzed with a one-way analysis of variance. Significant main effects $(\mathrm{p}<$ 0.05) were followed up with Fisher LSD post hoc comparisons. In addition, a ttest was used to compare $\mathrm{WL}$ and $\mathrm{WL}+\mathrm{VEHICLE}$ to determine if there was a vehicle effect. Sigmaplot 12.0 (San Jose, CA) was used for all statistical analyses. Values are reported as means $\pm \mathrm{SE}$, and significance for all tests was set at $p<0.05$. Exceptions to these statistical methods are stated in the figure legends. 


\section{RESULTS}

A t-test was performed between [WL vs. WL+VEHICLE] to determine whether the vehicle alone had an effect. WL and WL+VEHICLE showed no differences in any anthropometric measurement (Table 3.2 and Table 3.3), indicating that the vehicle, DMSO, did not affect these measurements in this study. In addition, a [WL vs. WL+VEHICLE] comparison was performed on hypothesis-specific transcripts (Table 3.1) and on all transcripts from the RNAseq analysis. No significantly different transcripts were identified $(\mathrm{WL}=$ WL+VEHICLE), indicating that the vehicle, DMSO, did not affect the PRAT transcriptome. Since the experimental purpose of this experiment was to investigate RUN and WL+GR BLOCK compared to $\mathrm{WL}$, and the usage of the WL+VEHICLE alone had no effect on WL, WL+VEHICLE was not included in the RNA-seq statistical analysis.

WL showed significantly greater increases in body mass, fat mass, and \% body fat when compared to RUN and WL+GR BLOCK (Table 3.2). Interestingly, while RUN equaled WL+GR BLOCK measures of adiposity (change in fat mass and change in \% body fat), RUN was greater than WL+GR BLOCK in increases in total body mass and change in lean mass, indicating that not only does the GR block attenuate adipose tissue growth, it attenuates increases in lean mass as well. PRAT mass was greatest in WL (WL was greater than RUN, while RUN was equal to $\mathrm{WL}+\mathrm{GR}$ BLOCK), PRAT adipocyte diameter was equal between WL and RUN (WL equaled RUN, while run was greater than WL+GR BLOCK). Taken together, these data suggest that the mechanisms differed by which RUN 


\section{and WL+GR BLOCK slow increases in PRAT mass seen with WL. Compared to}

\section{WL+GR BLOCK, RUN attenuates increase in total adipocytes while not differing}

in adipocyte diameter.

TABLE 3.2: Body mass, lean body mass, and $\%$ body fat, $n=6$ per group

\begin{tabular}{|c|c|c|c|c|}
\hline \multirow[b]{2}{*}{ Measurements } & \\
\hline & RUN & WL & WL+GR BLOCK & WL+VEHICLE \\
\hline Body mass day $70 \mathrm{~d}, \mathrm{~g}$ & $350.5 \pm 13.7$ & $346.5 \pm 3.6$ & $346.3 \pm 8.0$ & $346.3 \pm 10.5$ \\
\hline Body mass day $77 \mathrm{~d}, \mathrm{~g}$ & $364.8 \pm 13.9$ & $370.3 \pm 4.0$ & $349.6 \pm 8.4$ & $366.3 \pm 10.4$ \\
\hline Change in body mass (last 7 days), $\mathrm{g}$ & $14.3 \pm 0.6^{a}$ & $23.8 \pm 2.0^{b}$ & $3.3 \pm 0.8^{c}$ & $25.2 \pm 3.0^{b}$ \\
\hline Lean mass day $70 \mathrm{~d}, \mathrm{~g}$ & $324.0 \pm 10.0$ & $320.5 \pm 4.4$ & $318.6 \pm 8.1$ & $313.9 \pm 9.2$ \\
\hline Lean mass day $77 \mathrm{~d}, \mathrm{~g}$ & $338.6 \pm 10.7$ & $335.1 \pm 5.4$ & $321.9 \pm 9.0$ & $331.7 \pm 8.2$ \\
\hline Change in lean body mass (last 7 days), $\mathrm{g}$ & $14.7 \pm 1.9^{a}$ & $14.6 \pm 2.3^{a}$ & $3.3 \pm 1.3^{b}$ & $17.8 \pm 3.6^{\mathrm{a}}$ \\
\hline Fat mass day $70 \mathrm{~d}, \mathrm{~g}$ & $26.6 \pm 4.9$ & $26.0 \pm 2.7$ & $27.7 \pm 1.2$ & $27.2 \pm 1.3$ \\
\hline Fat mass day $77 \mathrm{~d}, \mathrm{~g}$ & $26.2 \pm 5.0$ & $35.1 \pm 4.0$ & $27.7 \pm 2.1$ & $34.6 \pm 4.3$ \\
\hline Change in Fat mass (last 7 days), $\mathrm{g}$ & $-0.4 \pm 1.9^{a}$ & $9.1 \pm 1.7^{\mathrm{b}}$ & $-0.1 \pm 1.1^{a}$ & $7.3 \pm 0.7^{b}$ \\
\hline Body fat $\%$ day $70 \mathrm{~d}, \mathrm{~g}$ & $7.4 \pm 1.1$ & $7.5 \pm 0.8$ & $8.0 \pm 0.4$ & $7.9 \pm 1.0$ \\
\hline Body fat $\%$ day $77 \mathrm{~d}, \mathrm{~g}$ & $7.0 \pm 1.1$ & $9.5 \pm 1.1$ & $8.0 \pm 0.6$ & $9.4 \pm 1.0$ \\
\hline Change in body fat $\%$ (last 7 days), $g$ & $-0.7 \pm 0.4^{a}$ & $2.0 \pm 0.4^{b}$ & $0.0 \pm 0.3^{a}$ & $1.5 \pm 0.3^{a}$ \\
\hline
\end{tabular}

Different letters denote between-group significant differences at $p<0.05$.

TABLE 3.3: Adipose tissue depot mass and adipocyte diameter, $n=6$ per group

\begin{tabular}{|c|c|c|c|c|}
\hline Measurements & RUN & WL & WL+GR BLOCK & WL+VEHICLE \\
\hline \multicolumn{5}{|l|}{ Epididymal Adipose Tissue Depot } \\
\hline Epididymal fat mass, $\mathrm{g}$ & $2.96 \pm 0.17$ & $4.04 \pm 0.44$ & $2.92 \pm 0.37$ & $3.67 \pm 0.35$ \\
\hline Epididymal Adipocyte diameter, $\mu \mathrm{m}$ & $64.8 \pm 3.6$ & $68.3 \pm 2.3$ & $60.3 \pm 1.7$ & $67.0 \pm 2.4$ \\
\hline Epididymal depot Cellularity $\left(\times 10^{6}\right)$ & $24.3 \pm 3.4$ & $26.3 \pm 1.8$ & $28.1 \pm 3.4$ & $31.3 \pm 2.8$ \\
\hline \multicolumn{5}{|l|}{ Perirenal Adipose Tissue Depot } \\
\hline Perirenal fat mass, $\mathrm{g}$ & $1.93 \pm 0.26^{\mathrm{a}}$ & $3.43 \pm 0.25^{b}$ & $1.80 \pm 0.28^{a}$ & $3.56 \pm 0.49^{b}$ \\
\hline Perirenal Adipocyte diameter, $\mu \mathrm{m}$ & $62.2 \pm 3.9^{a, b}$ & $67.1 \pm 1.4^{b}$ & $56.0 \pm 2.4^{a}$ & $69.6 \pm 3.0^{b}$ \\
\hline Perirenal depot Cellularity $\left(\times 10^{6}\right)$ & $14.9 \pm 1.1$ & $24.2 \pm 2.9$ & $20.7 \pm 2.0$ & $22.5 \pm 3.5$ \\
\hline \multicolumn{5}{|l|}{ Omental Adipose Tissue Depot } \\
\hline Omental fat mass, $\mathrm{g}$ & $0.35 \pm 0.02$ & $0.47 \pm 0.07$ & $0.43 \pm 0.06$ & $0.50 \pm 0.07$ \\
\hline Omental Adipocyte diameter, $\mu \mathrm{m}$ & $51.7 \pm 3.4^{\mathrm{a}, \mathrm{b}}$ & $54.9 \pm 2.4^{b}$ & $47.2 \pm 2.0^{a}$ & $57.7 \pm 1.4^{\mathrm{b}}$ \\
\hline Omental depot Cellularity (x 106) & $5.9 \pm 1.0$ & $5.8 \pm 0.5$ & $8.6 \pm 1.1$ & $6.9 \pm 0.4$ \\
\hline
\end{tabular}

A one-way ANOVA was performed on each outcome measure for all four groups. Different letters denote between-group significant differences at $p<0.05$. 


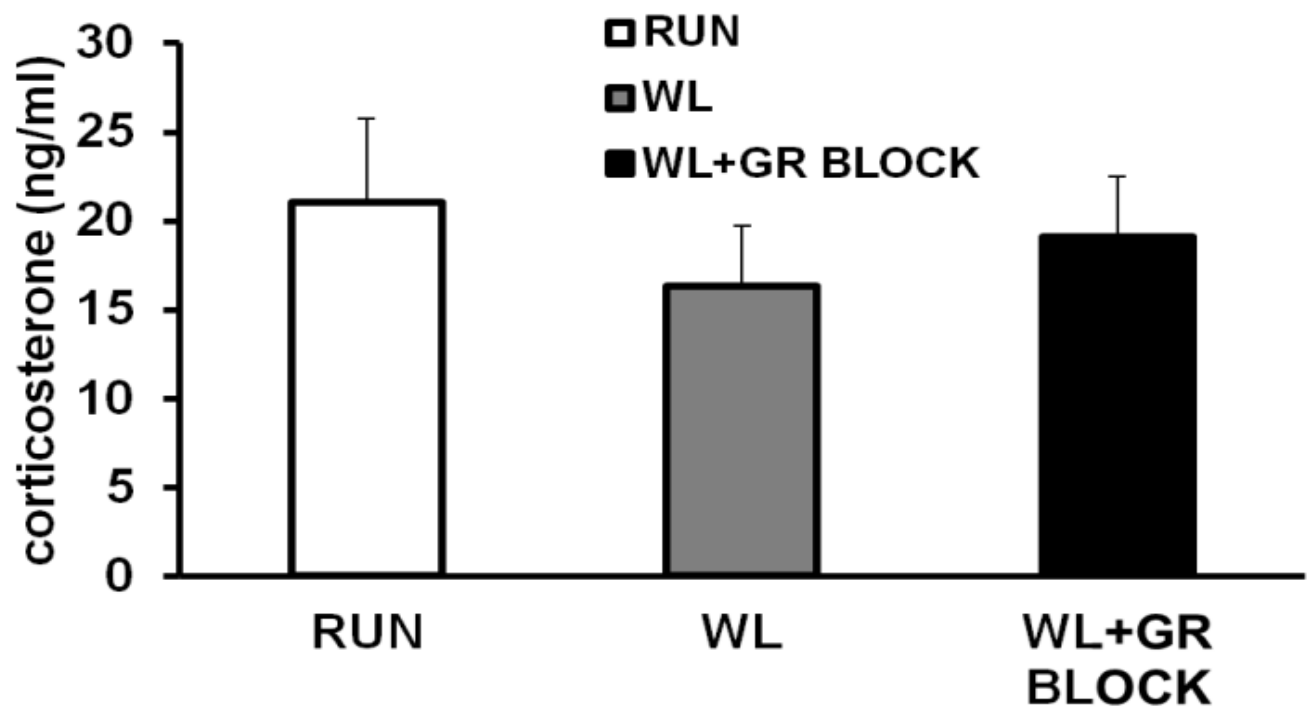

Figure 3.2. Corticosterone levels

There were no differences in corticosterone levels taken at the same time of day [139] (Fig. 3.2). Also, RNA-seq showed that the mRNA expression of GR was the same in all groups (average RPKM values: $R U N=42.9417 \pm 2.2140$, $W L=41.4198 \pm 1.7956$, and $W L+B L O C K=44.7859 \pm 1.2951)$. Together, for the measurements tested, group differences for the glucocorticoid system (plasma GCs and GR) were not evident.

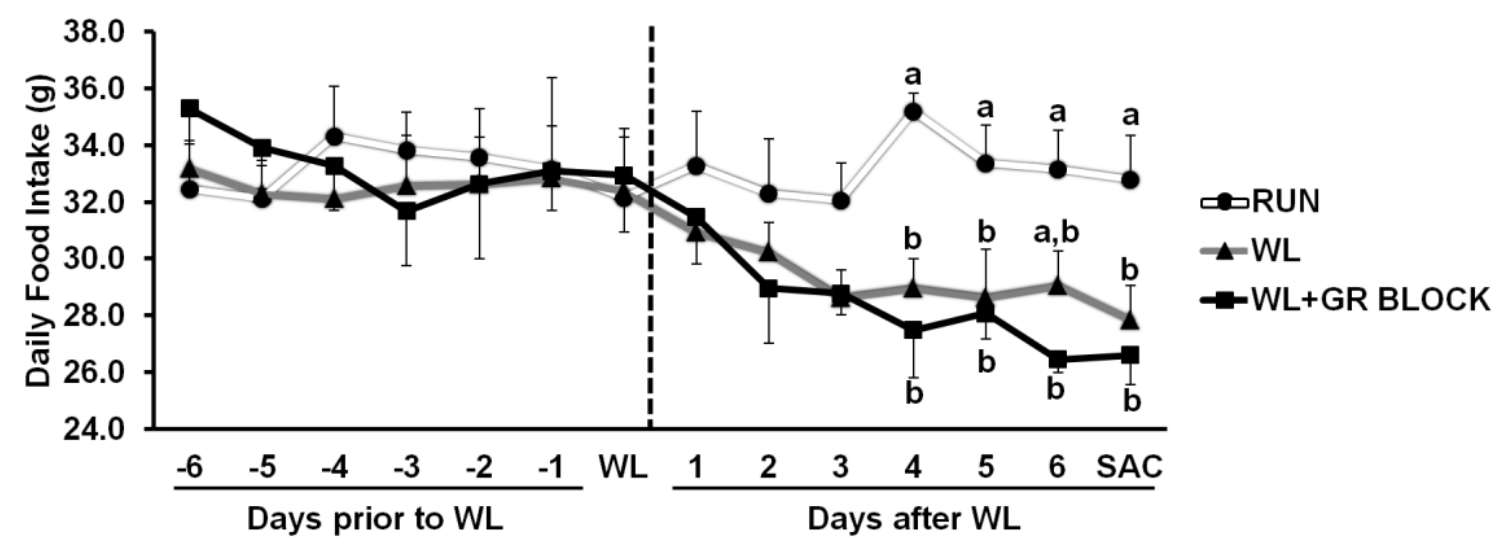

Figure 3.3: Daily food intake over the last 14 days

The vertical dashed like represents the day of WL. The WL rats represented in this table are a subset of the WL rats from data presented in Chapter 2. Different letters denote significance among groups at $p<0.05$. 
Food intake was similar among groups until 4 days after WL, at which time both the WL and WL+GR BLOCK groups ate less than RUN (Fig. 3.3). Importantly however, food intakes tended to start falling from their elevated levels on WL-day 1 , with the decrease accelerating daily to the significant decline on WL-day 4 for both WL and WL+GR BLOCK groups. Other than 6 days post WL, the $W L$ and $W L+B L O C K$ rats ate less than the RUN rats. Since $W L+B L O C K$ rats show the same trend in decreased food intake, this response to appetite to decreased activity holds true as the sample size enlarges. Taken together, WL is associated with a rapid decrease in appetite, even with GR block, indicating that increases in food intake are not responsible for increases in body fat with WL.

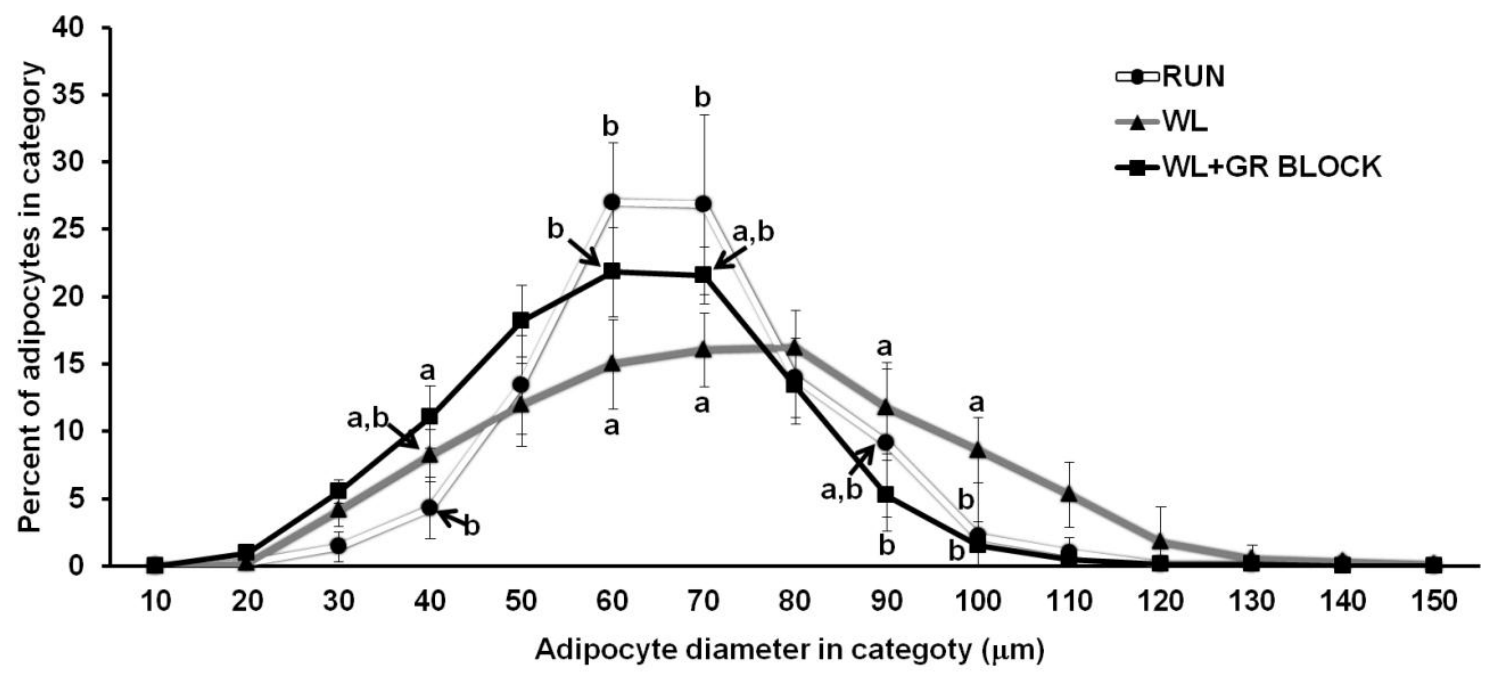

Figure 3.4. PRAT adipocyte size distribution

Adipocyte diameters were classified in 10- $\mu \mathrm{m}$ categories (10: 0.0-9.0 $\mu \mathrm{m}, 20: 10.0-19.9 \mu \mathrm{m}, 20: 20.0$ $29.9 \mu \mathrm{m}$, etc.) and the percentage of total adipocytes in a sample was plotted for each category. At least 300 adipocyte diameters were measured for each tissue sample. Different letters denote differences among groups at each category, $\mathrm{p}<0.05$.

Like $77 d$ 7d-WL data presented in Chapter 2, the adipocyte distribution curve for the WL group flattens out when compared to RUN (Fig. 3.4). 
Interestingly, the WL+GR BLOCK curve shows an intermediate response with less 'flattening' and more of a leftward shift compared to both WL and RUN. There were more adipocytes in the 30.0-39.9 $\mu \mathrm{m}$ category in WL+GR BLOCK than in RUN suggesting proliferation and differentiation of new adipocytes.

The aim of the first filter was to determine which transcripts were different between [RUN and $\mathrm{WL}]$, different between [WL+GR BLOCK vs. WL], and different between [RUN vs. WL+GR BLOCK]. The resulting differences for these three comparisons are presented next.

[RUN vs. WL]: 71 transcripts were significantly different at $p<0.05$, which corresponds to $41.0 \%$ of the 173 hypothesis-specific transcripts (Fig. 3.5A). Of these, 18 were up-regulated (25.4\%), and 53 were down-regulated (74.6\%) (Table 3.4). Of note, Cyclin A1 was down-regulated in RUN rats (fold change $=-$ 1.7229, $p=0.0016)$. This confirms that there are many DETs between RUN and WL in addition to Cyclin A1, thus confirming the conclusion from Chapter 2 that WL has increased proliferation compared to RUN.

[WL+GR BLOCK vs. WL]: 20 transcripts were significantly different at $\mathrm{p}<0.05$, which corresponds to $11.6 \%$ of the 173 hypothesis-specific transcripts (Fig. 3.5A). Of these, 9 were up-regulated (45.0\%), and 11 were down-regulated (55.0\%) (Table3.4). There were 5 transcripts that were up-regulated and 8 transcripts that were down-regulated in the $[R U N$ versus $\mathrm{WL}]$ and the [WL+BLOCK versus WL] comparison (Fig. 3.5A). These are noted in Table 3.4. 


\section{Transcripts Specific to Hypothesis}

A

RUN vs. WL

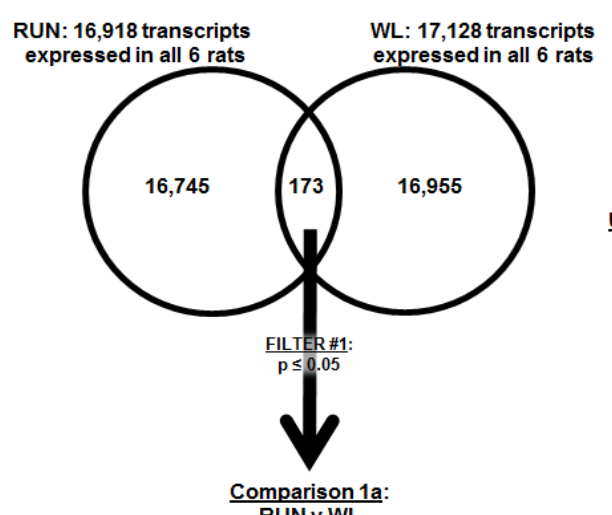

B

WL+GR BLOCK vs. WL

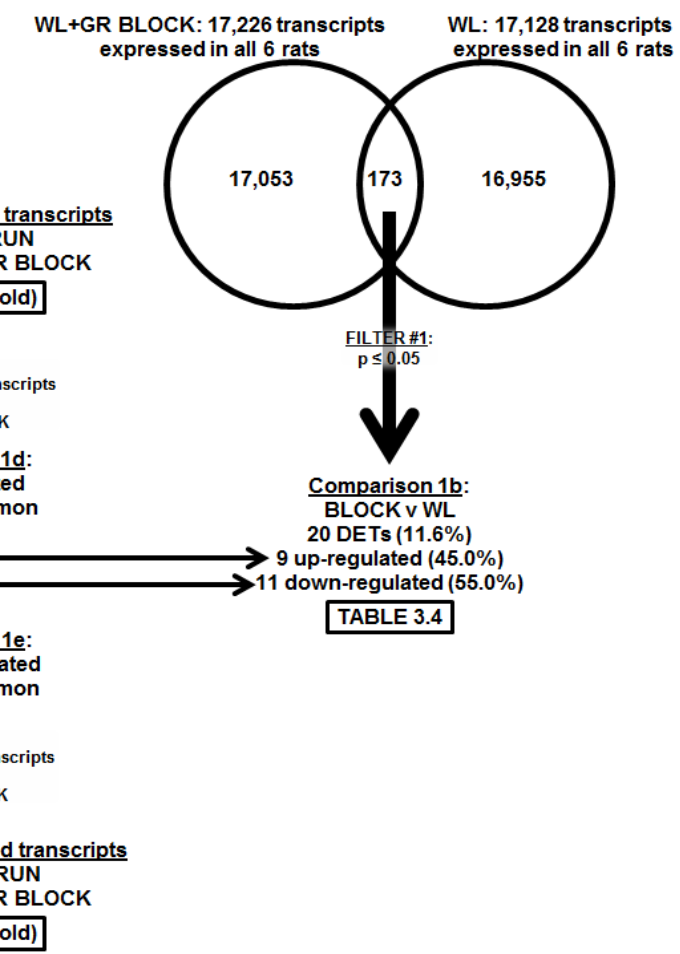

RUN v WL 18 up-regulated $(25.4 \%)$ 53 down-regulated $(\mathbf{7 4 . 6 \% )}$ TABLE 3.4

C

RUN vs. WL+GR BLOCK

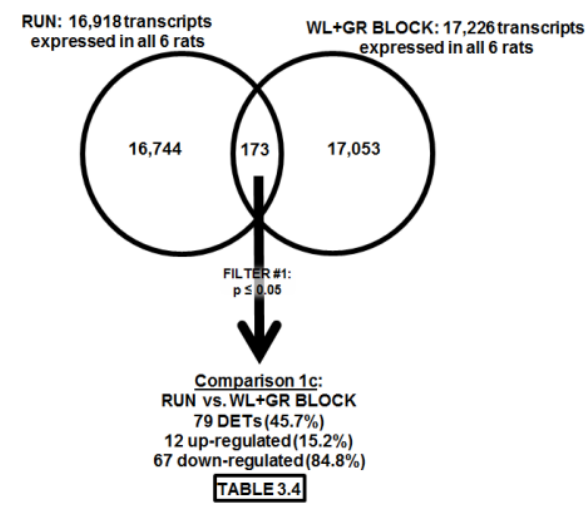

Figure 3.5. Hypothesis-specific RNA-seq results with filtering process to identify transcripts "unique to RUN" and "unique to BLOCK"

A list of 173 transcripts specific to the hypothesis was generated (see methods and Table 3.1). Three pairwise comparisons were performed: 1A, [RUN versus WL]; 1B, [BLOCK versus WL]; and 1C, [RUN versus WL+BLOCK]. FILTER \#1: transcripts for each of these pair-wise comparisons were considered significant if they were different at $p<0.05$. Figure 3.5A: 71 DETs were identified for [RUN versus WL] and 20 DETs were identified for [WL+BLOCK versus WL] (Table 3.4). The 18 up-regulated transcripts from [RUN versus WL] were compared to the 9 up-regulated transcripts from [WL+BLOCK versus WL] to identify common upregulated transcripts. Likewise, the 53 down-regulated transcripts from [RUN versus WL] were compared to the 11 down-regulated transcripts from [WL+BLOCK versus $\mathrm{WL}]$ to identify common down-regulated transcripts FILTER \#2: transcripts were omitted if the RPKM $\leq 1$ and if RUN = WL+BLOCK. Figure 3.5B: 79 DETs were identified for [RUN versus WL+BLOCK]; 12 were up-regulated, and 67 were down-regulated. This comparison was used to directly address the hypothesis as well as significance criteria in FILTER \#2. 
[RUN vs. WL+GR BLOCK]: 79 transcripts were significantly different at $p<0.05$, which corresponds to $45.7 \%$ of the 173 hypothesis-specific transcripts (Fig. 3.5B). Of these, 12 were up-regulated (15.2\%), and 67 were downregulated (84.8\%) (Table 3.4).

The aim of the second filter was to determine which hypothesis-specific transcripts were uniquely up- and down-regulated to RUN and to BLOCK (Fig. 3.5). These hypothesis-specific transcripts are presented next.

Unique to RUN, up-regulated: of the 18 up-regulated hypothesis-specific transcripts, the 5 transcripts that were also up-regulated in the [WL+GR BLOCK vs. WL] analysis were omitted. Of the remaining 13 transcripts, 5 were omitted because they were not different from WL+GR BLOCK. The 8 remaining transcript is highlighted in bold in Table 3.4. These 8 transcripts were entered into IPA analysis, and the top up-regulated network is shown in Figure 3.6A.

Unique to RUN, down-regulated: of the 53 down-regulated hypothesisspecific transcripts, the 8 transcripts that were also down-regulated in the [WL+GR BLOCK vs. WL] analysis were omitted. Of the remaining 45 transcripts, 6 were omitted due to the RPKM values $\leq 1$, and 11 were omitted because they were not different from $\mathrm{WL}+\mathrm{BLOCK}$. The 28 remaining transcript is highlighted in bold in Table 3.4. These 28 transcripts were entered in IPA analysis, and the top down-regulated network is shown in Figure 3.6B. 
Unique to WL+GR BLOCK, up-regulated: of the 9 up-regulated hypothesis-specific transcripts, the 5 transcripts that were also up-regulated in the $[R U N$ vs. WL] analysis were omitted. Of the remaining 4 transcripts, 2 were omitted because they were not different from RUN. The remaining 2 transcripts are highlighted in bold in Table 3.4 .

Unique to WL+GR BLOCK, down-regulated: of the 11 down-regulated hypothesis-specific transcripts, the 8 transcripts that were also down-regulated in the $[R U N$ vs. $W L]$ analysis were omitted. Of the remaining 3 transcripts, one was omitted because its RPKM value was less than 1, and one was omitted because it was not different from RUN. The remaining transcript is highlighted in bold in Table 3.4. Interestingly, Angptl4 (up-regulated in WL+GR BLOCK), Smc2 and Cdk6 (both down-regulated in RUN) were identified as significant transcripts in the entire RNA-seq analysis (Fig. 3.7), thus identifying these three transcripts as candidates for future hypotheses.

My a priori hypothesis was not confirmed. When compared to WL, RUN and WL+GR BLOCK do not show similar changes in PRAT transcript expression involved in cell cycle, proliferation, and differentiation. Two tests were performed that tested my pre-hoc hypothesis (see introduction and Fig. 3.5A \& 3.5B). Test 1 compared hypothesis-specific transcripts between RUN and WL+GR BLOCK and showed that there were 79 DETs (45.7\%) between these groups. Had there been zero DETs between these groups, our hypothesis would have been confirmed. Test 2 compared hypothesis-specific transcripts between [RUN vs. WL] and [WL+GR BLOCK vs. WL] and did not show similar DETs (RUN: $27.8 \%$ 
of up-regulated DETs and $15.1 \%$ of down-regulated DETs were common to WL+GR BLOCK; WL+GR BLOCK: $55.6 \%$ of DETs up-regulated DETs and $72.7 \%$ of down-regulated DETs were common to RUN). Had all of the DETs been the same between these comparisons, the hypothesis would have been confirmed.

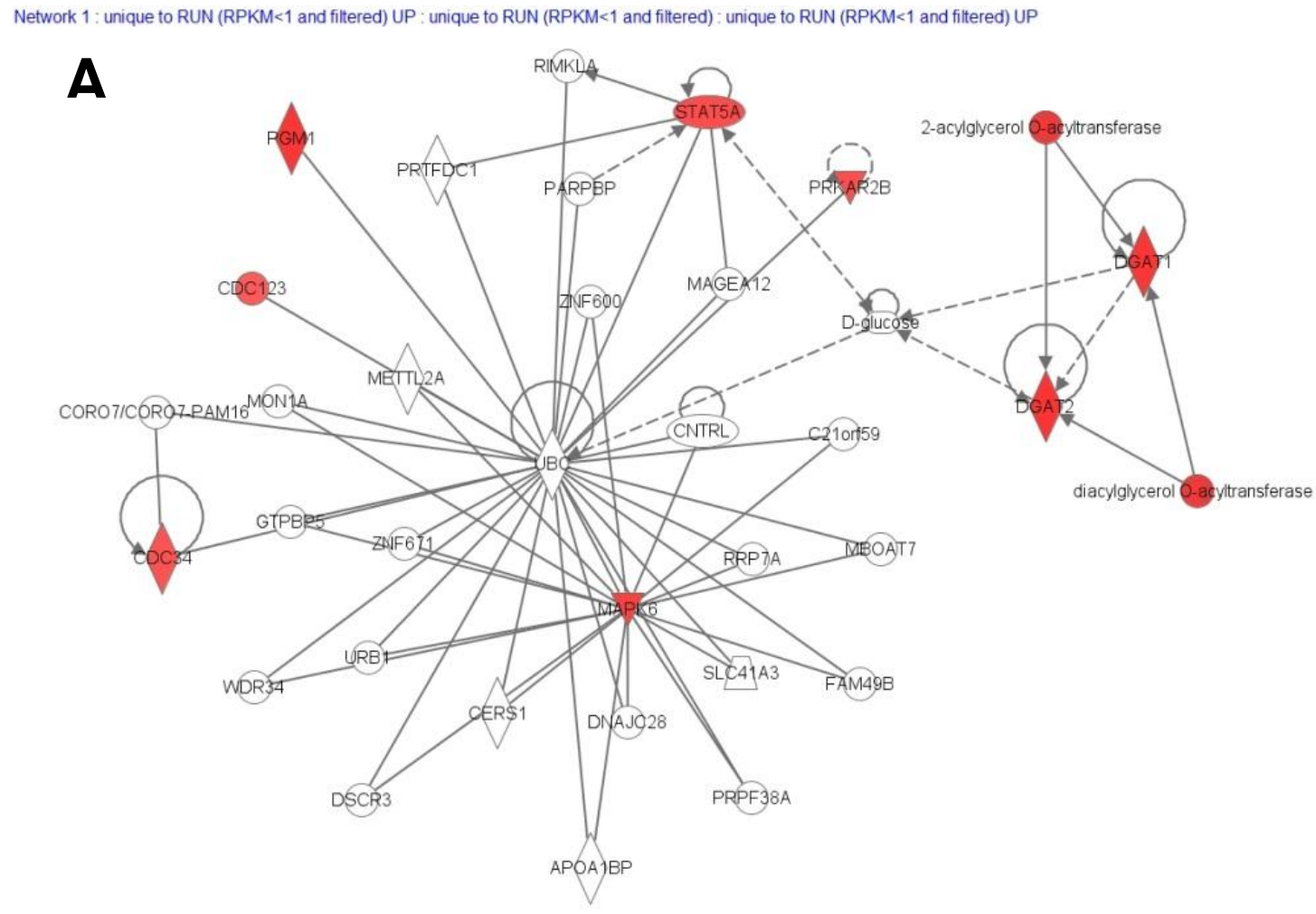


Network 1 : unique to RUN (filters ..) DOWN : unique to RUN (filters ...) DOWN : unique to RUN (filters ..) DOWN

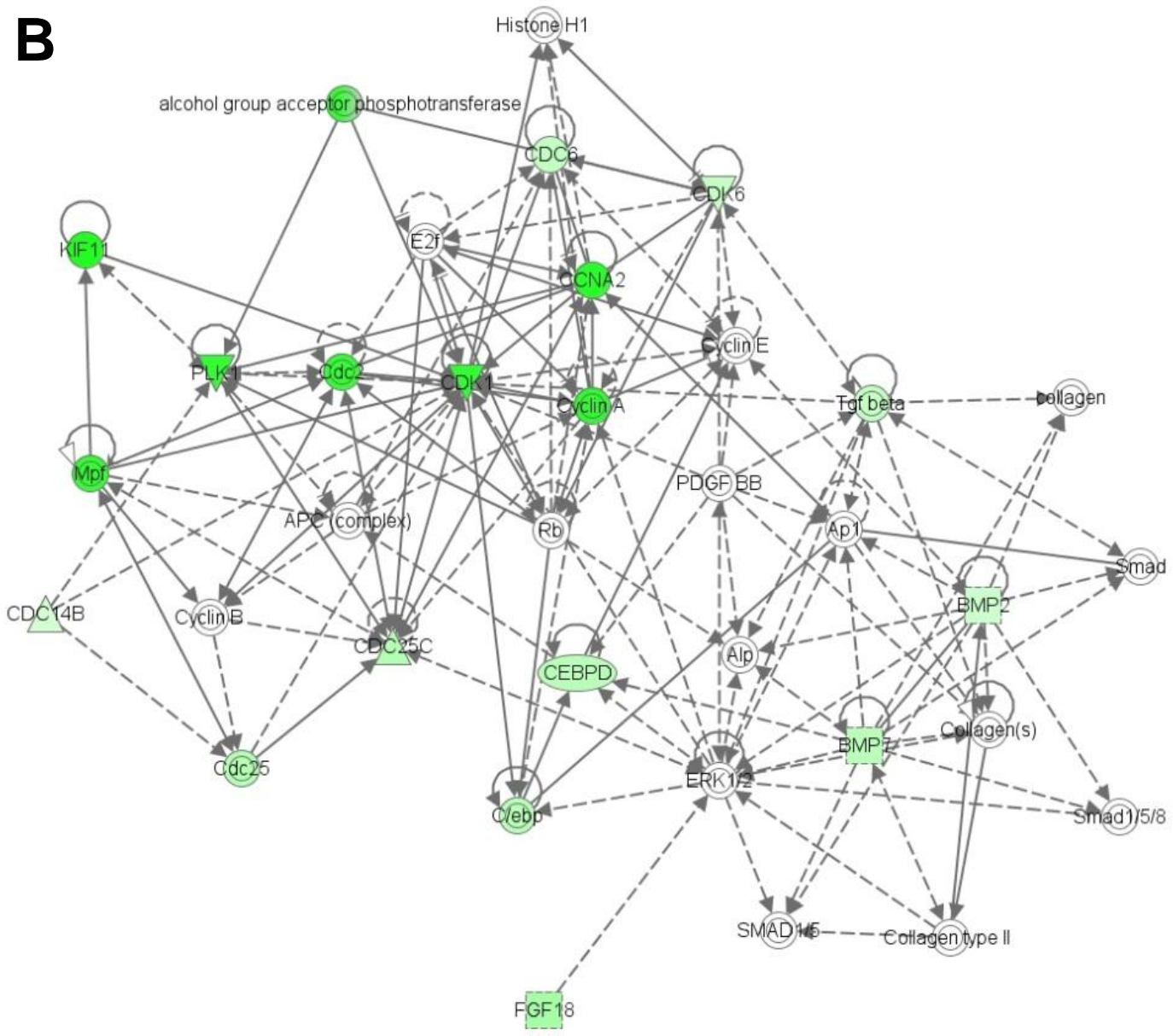

() 2000-2013 Ingenuity Systems, Inc. All rights reserved

Figure 3.6. Top scoring up- and down-regulated networks for transcripts unique to RUN

A: IPA-derived top-scoring network, "Lipid Metabolism, Molecular Transport, Small Molecule Biochemistry" (Table 3.4) produced from a list of 8 transcripts uniquely up-regulated in RUN rats. The red colored nodes represent up-regulated genes. B: IPA-derived top-scoring network, "Cell Cycle, Cell Death and Survival, Tumor Morphology" (Table 3.4) produced from a list of 28 transcripts uniquely down-regulated in RUN rats. Green colored nodes represent down-regulated genes. 


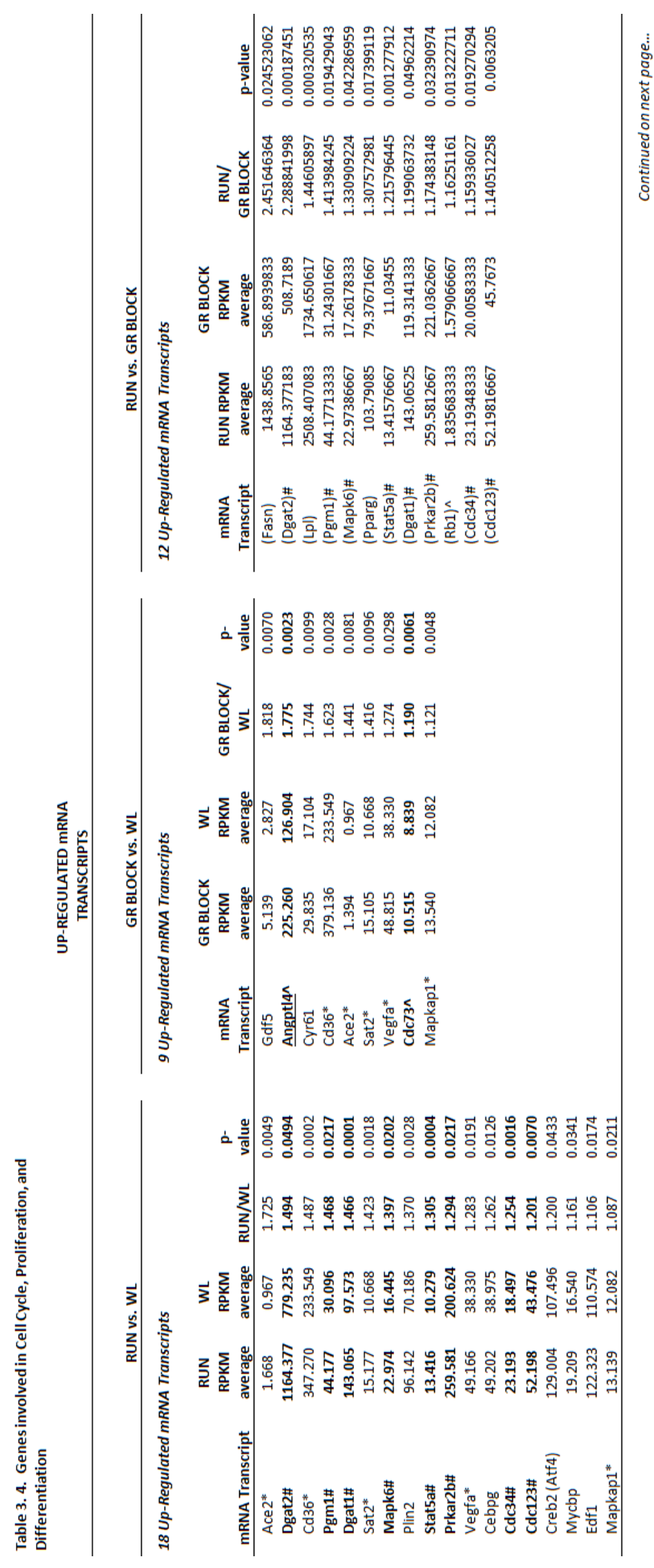




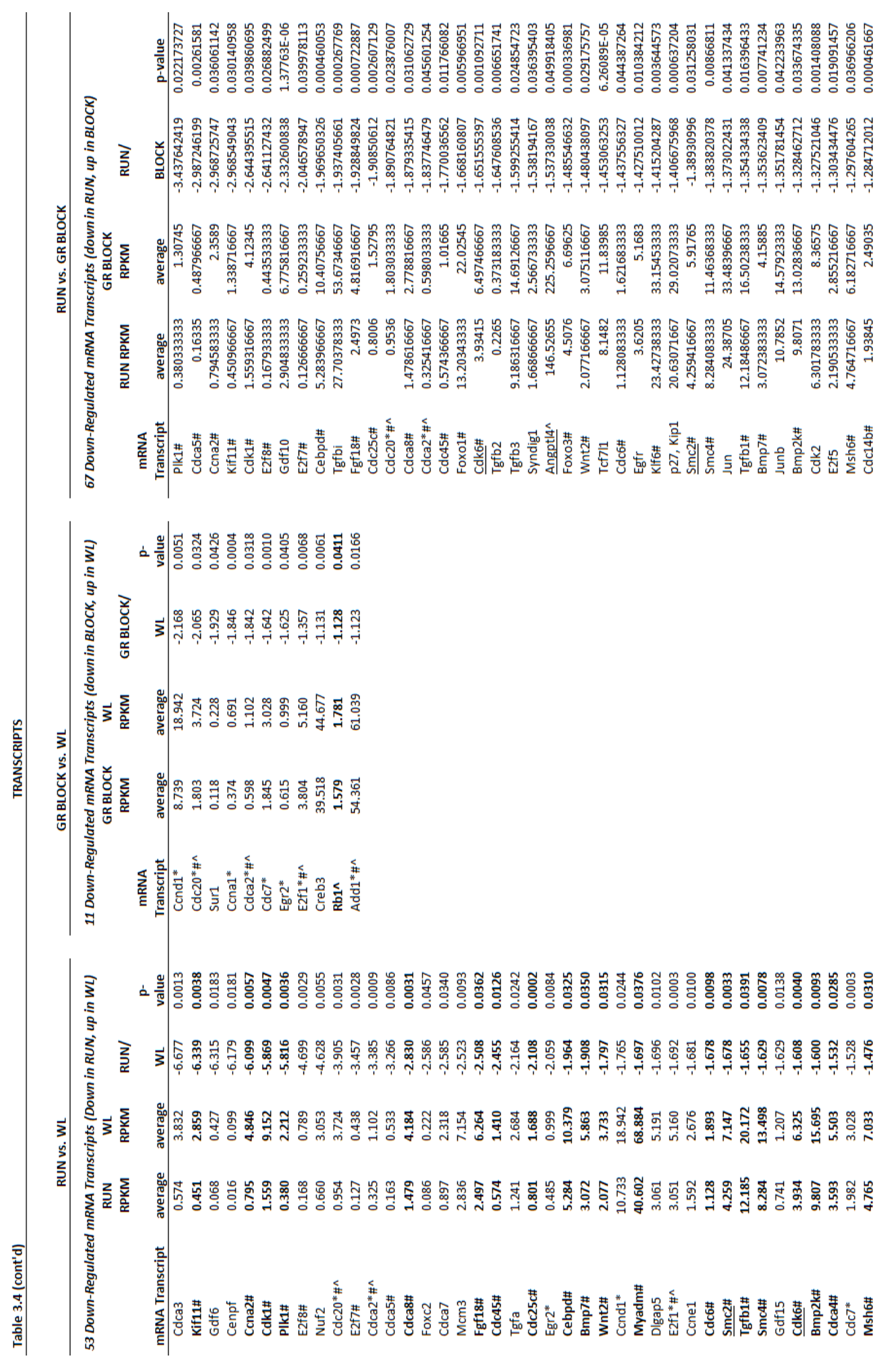




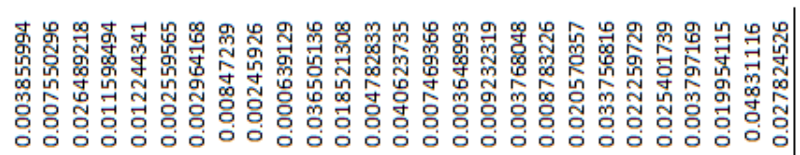

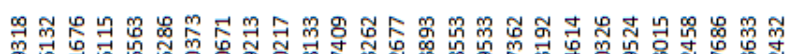

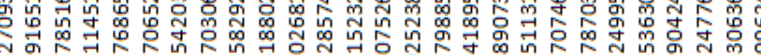

๙

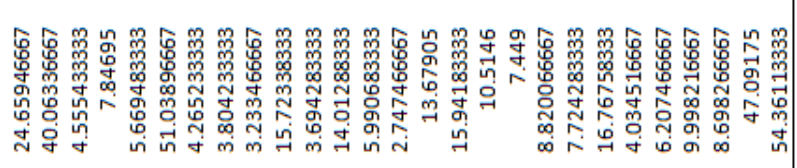

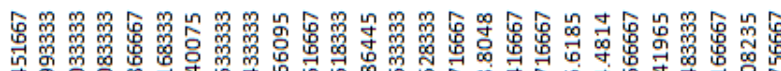

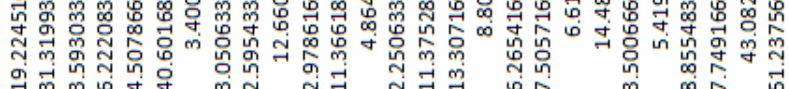

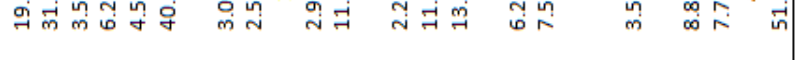

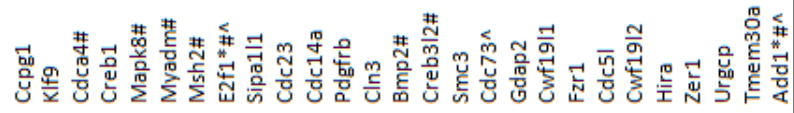

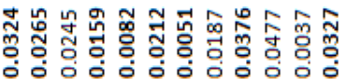

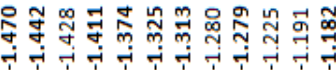

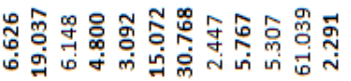

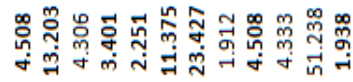

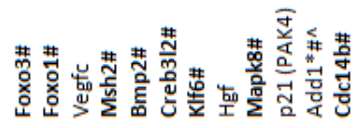

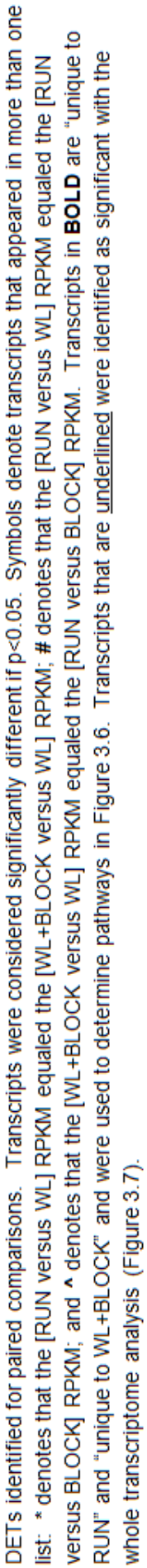




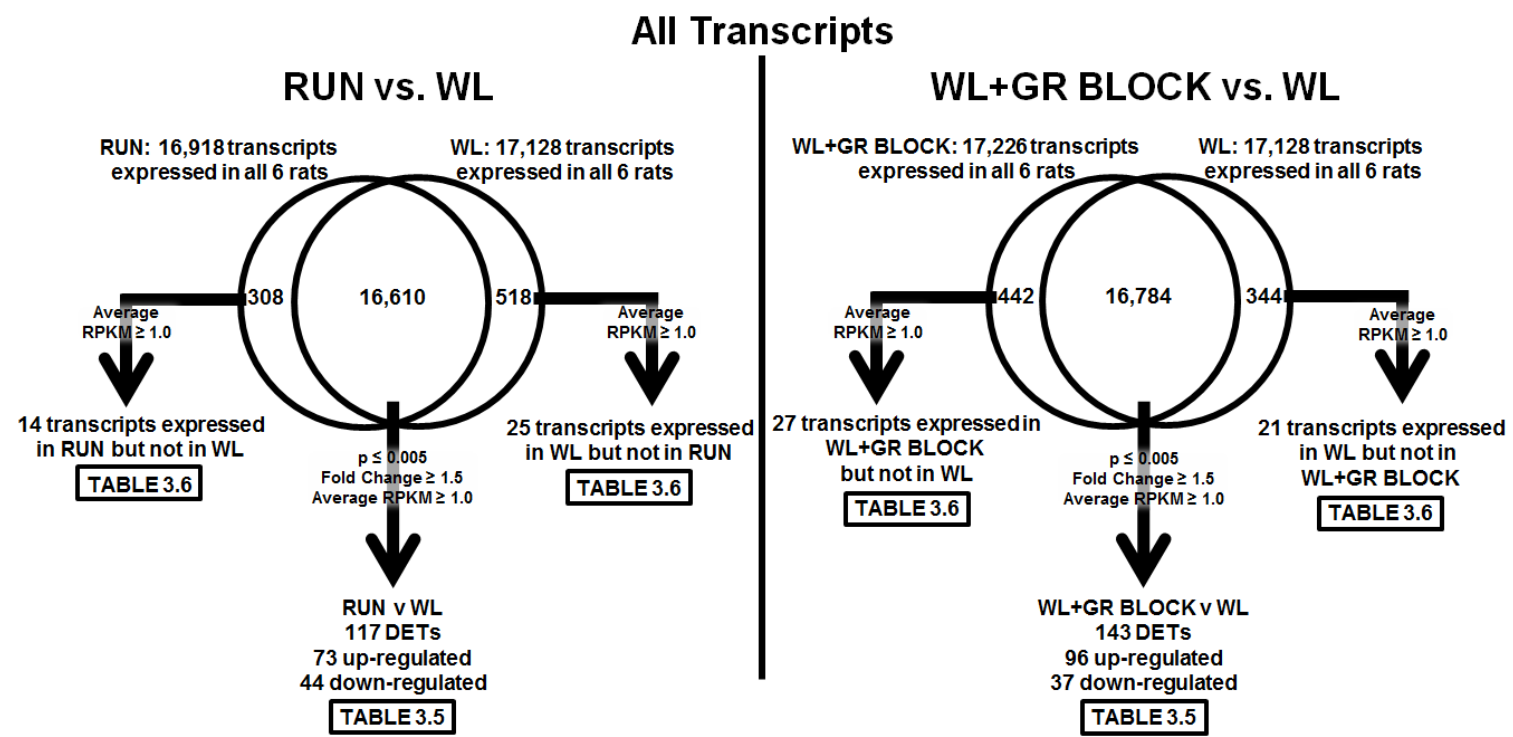

Figure 3.7: Whole transcriptome RNA-seq filtering process and results

For each group, a transcript was omitted if it was not known and not expressed in each member of that group. There were 16,918 RUN, 17,128 BLOCK, and 17,226 WL+BLOCK transcripts remaining after this. Two comparisons were performed: [RUN versus $W L]$ and [WL+BLOCK versus $W L]$. For the [RUN versus WL] comparison, there were 16,610 commonly expressed genes that were compared and filtered for significance. A transcript was deemed significantly different between RUN and WL if: 1) $p<0.005$, 2) fold change $\geq 1.5$, and 3 ) average RPKM $\geq 1$. 117 transcripts met these criteria. Of these 118 transcripts, 73 were up-regulated and 44 were down-regulated. The top- 10 transcripts and top IPA network for each are listed in Table 3.5. There were 308 transcripts that were expressed in RUN but not in WL. Of these, 14 transcripts had a RPKM $\geq 1$. The top-10 transcripts and top network are listed in Table 3.6. There were 518 transcripts that were expressed in WL but not in RUN. Of these, 25 transcripts had a RPKM $\geq 1$. The top10 transcripts and top network are listed in Table 3.6. For the [WL+BLOCK versus WL] comparison, there were 16,784 commonly expressed genes that were compared and filtered for significance using the same criteria as described above. 143 transcripts met these criteria. Of these 143 transcripts, 96 were upregulated and 37 were down-regulated. The top-10 transcripts and top IPA networks for each are listed in Table 3.5. There were 442 transcripts that were expressed in WL+BLOCK but not in WL. Of these, 27 had a RPKM $\geq 1$. The top-10 transcripts and top IPA network are listed in Table 3.6. There were 334 transcripts that were expressed in WL but not in WL+BLOCK. Of these, 21 had a RPKM $\geq 1$. The top-10 transcripts and top IPA network are listed in Table 3.6. The entire list of transcripts identified in the RUN vs. WL comparison can be found in Appendix A.

To observe the entire PRAT transcriptome without limiting the analysis to cell cycle, proliferation, and differentiation transcripts, RNA-seq analysis was performed using a different filtering process. The rationale for including this data here is to show the "wealth" of data that can be analyzed in the future. Top transcripts and pathways are identified in Table 3.5 \& 3.6. 


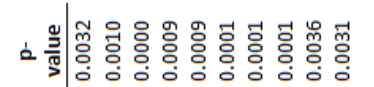

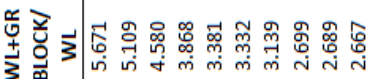

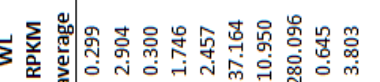

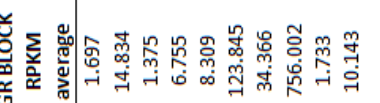
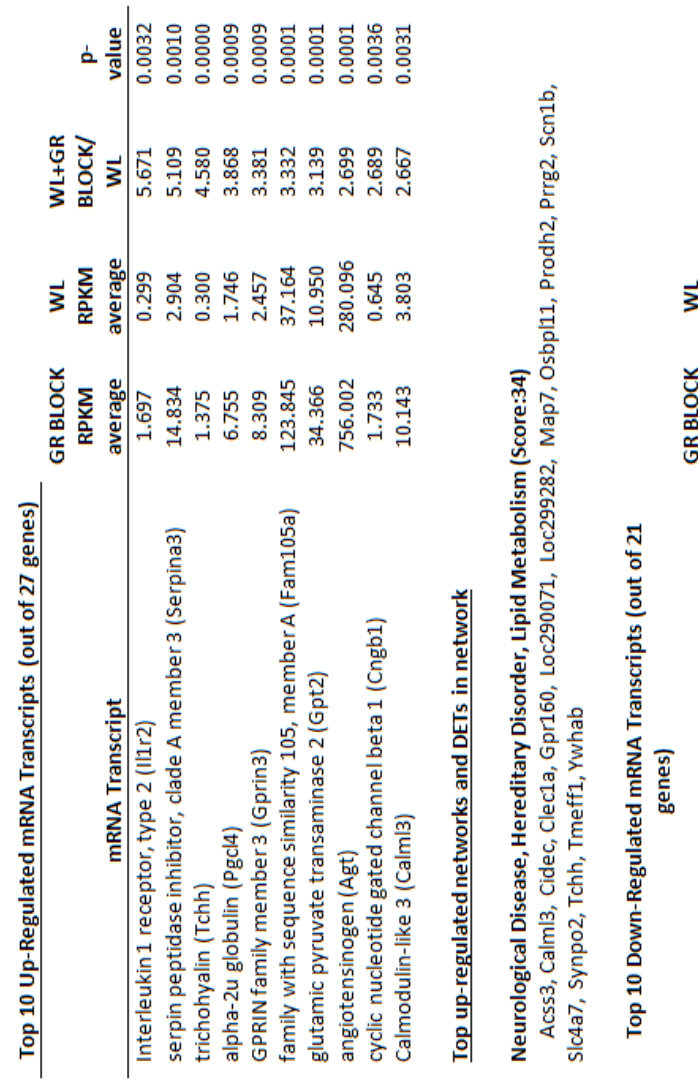

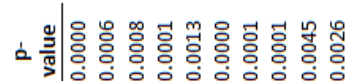

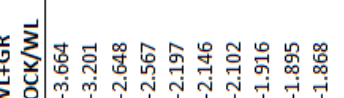

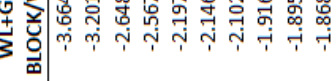

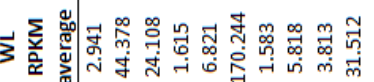

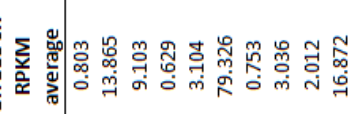

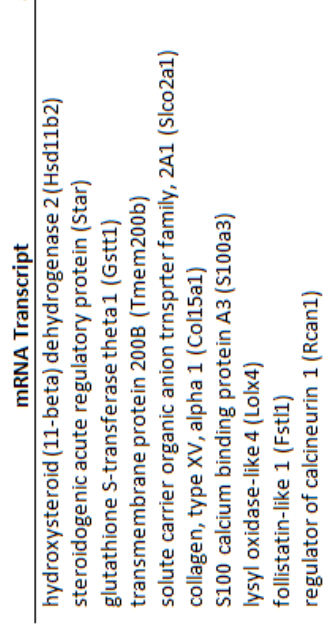

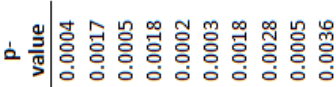

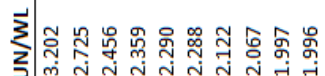

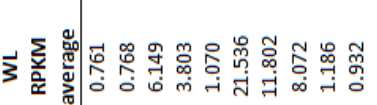

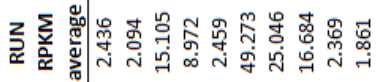
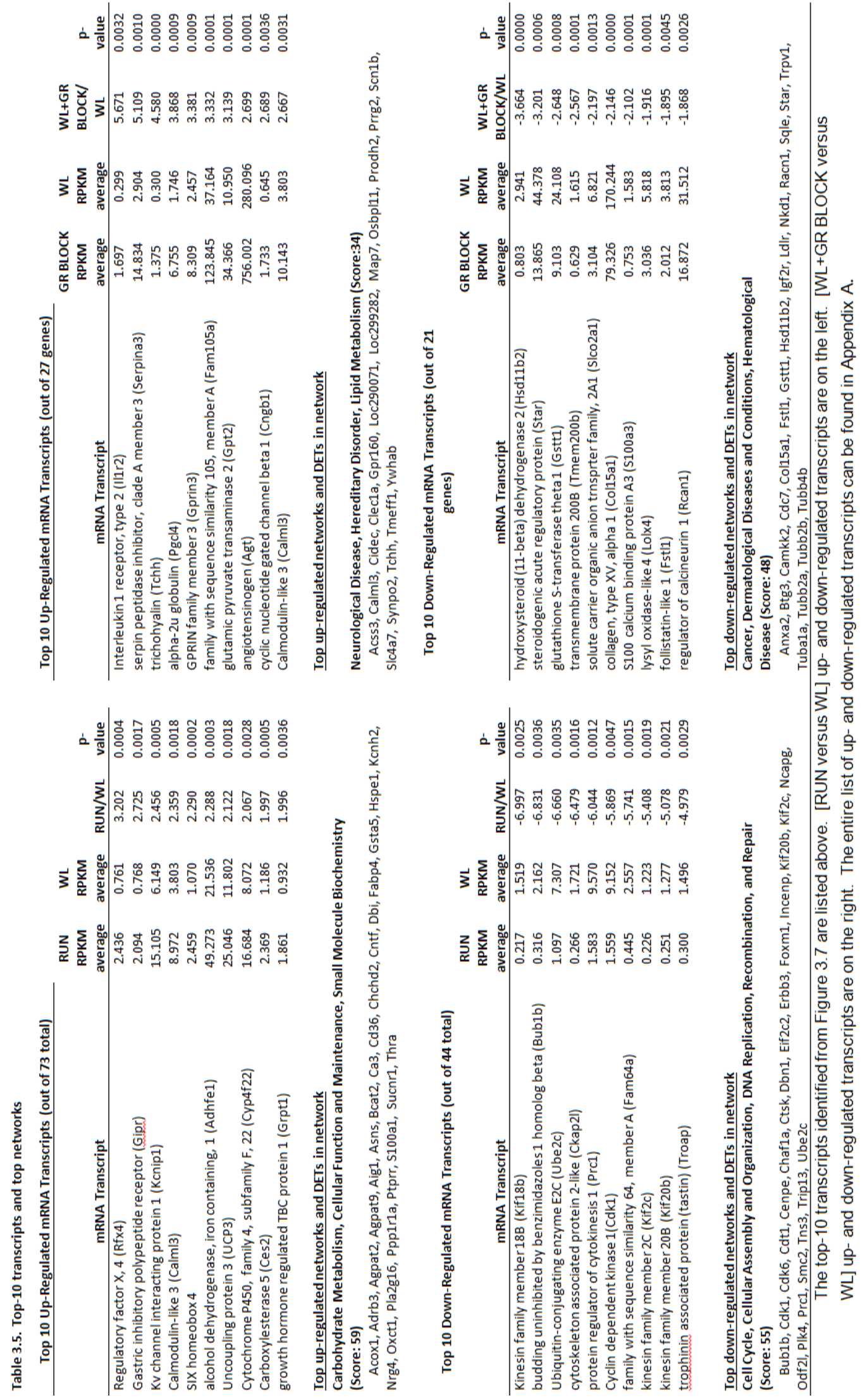

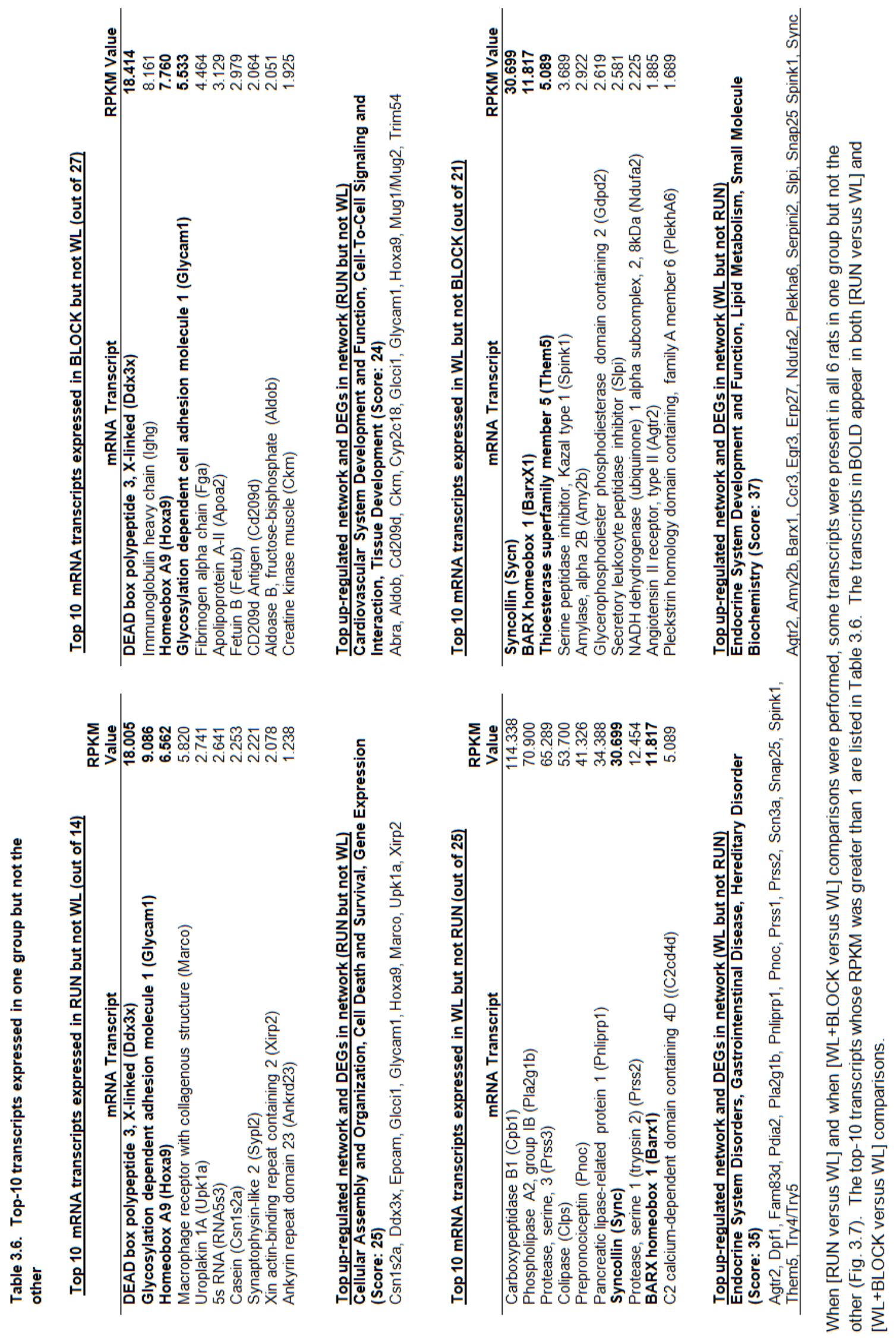


\section{DISCUSSION}

The primary purpose of this experiment was to determine whether RUN and GR block had similar transcriptional profiles for genes involved in cell cycle, proliferation, and differentiation. Based on this preliminary data and data from other studies [[60] and Chapter 2], my overall hypothesis for this study is that, when compared to WL, RUN and WL+GR BLOCK would show similar changes in PRAT transcript expression involved in cell cycle, proliferation, and differentiation. I tested this hypothesis two ways:

1) If a comparison of hypothesis-specific transcripts between RUN and WL+GR BLOCK show similar up- and down-regulation, then we can conclude that these two conditions express similar transcript changes and probably affect cell cycle, proliferation, and differentiation in the same manner. But if there are many differentially expressed hypothesis-specific transcripts between RUN and WL+GR BLOCK, then we can conclude that these two conditions do not affect cell cycle, proliferation, and differentiation in the same manner, and RUN and WL+GR BLOCK likely keep fat from accumulating by different mechanisms.

2) If the DETs for the hypothesis-specific genes for the comparison of [RUN vs. $\mathrm{WL}]$ and the comparison of [WL+BLOCK vs. WL] are the same, then we can conclude that these two conditions affect transcripts related to cell cycle, proliferation, and differentiation when compared to WL similarly and probably work to keep fat from accumulating in the same manner. But if there are many DETs identified for the comparison of [RUN vs. WL] that differ from the 
comparisons of [WL+BLOCK vs. WL], we can conclude that these two conditions do not affect cell cycle, proliferation, and differentiation in the same manner and likely keep fat from accumulating by different mechanisms.

If the hypothesis was accepted, then further analysis will be performed on the top transcripts that were similarly expressed in RUN and WL+GR BLOCK to identify a common pathway involved with suppression of fat accumulation with WL and to identify candidate genes for potential future studies. If the hypothesis was not accepted, then the top up-regulated and top down-regulated genes unique to RUN (not up/down-regulated in WL+GR BLOCK) and to WL+GR BLOCK (not up/down-regulated in RUN) would be identified for analysis on potential key transcripts and pathways unique to RUN and to WL+GR BLOCK. These unique transcripts could help identify differing mechanisms by which RUN and WL+GR BLOCK attenuate the increase in fat with WL. Additionally, these hypothesis-specific transcripts would test the hypothesis presented at the end of Chapter 2 that "perirenal adipose tissue is expanding by hyperplasia due to an increase in cell cycle, proliferation, and differentiation." Finally, RNA-seq data from all transcripts was analyzed in order to observe other non-cell cycle, proliferation, and differentiation pathway transcriptomic differences within PRAT that existed among RUN, WL, and WL+BLOCK in order to generate new hypotheses for future experiments using this model. 
Two separate analyses of transcripts involved in cell cycle, proliferation, and differentiation showed that, contrary to the hypothesis, RUN and $W L+G R$ BLOCK express different transcripts. We concluded that these two conditions did not affect cell cycle, proliferation, and differentiation in the same manner. We then identified which transcripts differed between RUN and WL+GR BLOCK to identify potential key transcripts and pathways unique to $R U N$ and to $W L+G R$ BLOCK to generate new hypotheses. A secondary purpose of this experiment was to confirm the findings in Chapter 2 and provide more molecular support that WL increases PRAT by increased molecular markers of cell cycle, proliferation, and differentiation. We conclude that indeed, PRAT from WL animals show many changes ( $41.0 \%$ of the 173 hypothesis-specific genes differed) in cell cycle, proliferation, and differentiation genes when compared to RUN.

RUN and WL+GR BLOCK groups failed to attain levels of fat mass, $\%$ body fat, and PRAT depot mass associated with WL. However WL+GR BLOCK also prevented gains in both total body mass and lean body mass, which were present in WL and RUN groups. This is consistent with the literature in that RU486 has been shown to attenuate body mass gains in rodents [145] and in humans [146]. However, it is interesting that this occurred since increased GC levels in skeletal muscle leads to decreased protein synthesis and increased proteolysis. Patients with excess GC often present muscle atrophy and weakness [147], and contrary to our findings, in the presence of excessive GCs, RU486 prevents muscle atrophy [148]. The rats in this study did not exhibit excess cortiosterone and, to our knowledge, were not experiencing GC-induced 
muscle atrophy. WL+GR BLOCK rats had a similar PRAT mean adipocyte diameter as RUN, but less than WL. However, WL+GR BLOCK rats had statistically the same estimated number of adipocytes as WL. This observation was also present in the adipocyte size distribution curve. WL+GR BLOCK rats did not exhibit a flattening of the curve to the same extent as WL. Rather, the curve was slightly shifted to the left and there were more adipocytes in the 30.0$39.9 \mu \mathrm{m}$ category in $\mathrm{WL}+\mathrm{GR}$ BLOCK than in RUN, suggesting that $\mathrm{WL}+\mathrm{GR}$ BLOCK either had proliferation and differentiation of new adipocytes or a lipolysis and 'shrinking' of pre-existing adipocytes. Regarding the possibility of increased lipolysis in WL+GR BLOCK, enzymes involved in triacylglycerol breakdown include adipose triglycerol lipase (AGTL), hormone sensitive lipase (HSL), and monoglycerol lipase (MGL) were evaluated from RNA-seq data. AGTL, HSL, and MGL were not different between RUN and WL+GR BLOCK. However, ATGL was up-regulated in [RUN vs. WL] (fold change $=1.3280, p=0.0196$ ) and HSL was up-regulated in [WL+GR BLOCK vs. WL] (fold change $=1.2927$, $p=0.043$ ). The last observation offers some support to the notion of smaller adipocytes in WL+GR BLOCK compared to WL due to increased lipolysis.

We did not see a difference in corticosterone levels in any group. GCs are often discussed in the context of Cushing's syndrome, where there is an excess of circulating GCs with patients presenting with central obesity symptoms similar to patients with common metabolic syndrome [149]. Unlike Cushing's syndrome, in common obesity, systemic GC levels do not change $[126,150]$ and may be lower than in lean individuals [151], suggesting that elevated systemic cortisol 
levels are not the cause of WL-induced increases in abdominal adiposity. Interestingly, GCs affect different fat depots differently; they increase lipolysis in peripheral fat depots, but they promote pre-adipocyte differentiation and adipocyte hypertrophy in VAT [131], so there is something unique to VAT for response to GC action.

Given the important role of the enzyme $11 \beta \mathrm{HSD}-1$ in adipose tissue regarding visceral obesity and metabolic health (see Chapter 1 and Chapter 4 and $[126,152-155]$, it is worth mentioning that WL+GR BLOCK up-regulated $11 \beta$ HSD-1 compared to RUN (1.4031, $p=0.025)$ and compared to WL (fold change $=1.4627, p=0.034$ ). Expression in RUN did not differ from WL (fold change $=1.0425, p=0.749)$. Interestingly, $11 \beta \mathrm{HSD}-2$ was the top downregulated transcript identified between [WL vs. WL+GR BLOCK] (fold change = 3.6637, $p=0.00002)$. In the context of RUN versus $W L$, the fact that the expression of neither of these isoforms differed indicates that this GCactivating/inactivation pathway is not critical to adipose tissue homeostasis in our model. However, when GC action is attenuated, such as with the GR block, the up-regulation of $11 \beta \mathrm{HSD}-1$ may indicate that $11 \beta \mathrm{HSD}-1$ acts as a compensatory mechanism to increase GC activity in PRAT.

\section{RNA-sequencing}

The remainder of the discussion will address RNA-seq analysis on transcripts specific to the pre-hoc hypothesis, specifically particular transcripts that were unique to RUN and to WL+GR BLOCK (Fig. 3.5 and Table 3.4), with 
hopes that these transcripts will provide insight into the differing mechanisms by which RUN and WL+BR BLOCK attenuate fat gains seen with WL. Speculation and discussion on transcripts identified in the entire RNA-seq analysis (Fig. 3.7) is not presented here. The purpose of showing data from the entire RNA-seq transcriptome in the results section was to show that there are additional transcripts and pathways that can be addressed from this RNA-seq dataset in future studies.

Previous RNA Sequencing studies categorized gene expression levels into three groups: "low expressed transcripts" are between 0.125-0.999 RPKM average, "medium expressed transcripts" are between 1.0 and 10.0 RPKM average, and "high expressed transcripts" are above 10.0 RPKM average [142]. All transcripts classified as "low expressed" were omitted in the filtering process. The rationale for this decision was that transcripts expressed between 1.0-3.0 RPKM are equivalent to a single gene copy per cell [141]. If a transcript is expressed at a level less than one per cell, it is questionable if the transcript actually exerts an effect. The RPKM values for transcripts discussed below are provided, thus allowing speculation to the potential magnitude of these transcripts. Overall, my interpretation of the following findings are that RUN limited cell cycle progression, proliferation, and differentiation compared to WL+GR BLOCK rats. 


\section{Transcripts unique to RUN.}

There were 8 transcripts unique to RUN that were up-regulated and 28 transcripts unique to RUN that were down-regulated. These transcripts were entered into an IPA analysis and the top pathways were identified. A limitation of IPA is that pathway names are IPA's and sometimes their names are broader than our data. The unique transcripts for both up- and down-regulation will be discussed in the context of the top pathway for both up- and down-regulation. In addition to the transcripts in the top down-regulated pathway, two additional interesting down-regulated transcripts will be discussed.

\section{Transcripts uniquely up-regulated in RUN.}

IPA analysis identified "Lipid Metabolism, Molecular Transport, Small Molecule Biochemistry" as the top pathway given, which happened to include all 8 transcripts: diacylglycerol O-acyltransferase 1 (Dgat1), diacylglycerol Oacyltransferase 2 (Dgat2), protein kinase, cAMP-dependent, regulatory, type II, beta (Prkar2b), signal transducer and activator of transcription 5a (Stat5a), cell division cycle 34 homolog (Cdc34), cell division cycle 123 homolog (Cdc123), phosphoglucomutase 1 (Pgm1), and mitogen-activated protein kinase 6 (Mapk6) (Fig. 3.6A).

Dgat1 and Dgat 2 (diacylglycerol O-acyltransferase 1 and 2) are highly expressed in PRAT (RPKM average $=117.1175$ and 854.678, respectively, $n=24)$ and mRNA expression has been shown to be down-regulated in the presence of an active-GC suppressor [156], suggesting that Dgat expression is 
regulated by GCs. DGATs catalyzes the final reaction of triglyceride synthesis and is a characteristic of a fully differentiated adipocyte. In fact, overexpression of Dgat1 reduces cell growth rate and proliferation [157], while Dgat1 knock-out mice are resistant to obesity [158], have increased sensitivity to insulin and leptin, and are resistant to diet-induced hepatic steatosis [159]. Dgat1 mRNA expression increases in skeletal muscle of mice and humans after an acute bout of exercise yet is unchanged after chronic exercise training [160], but there is no previous data to our knowledge on adipose tissue and exercise. Dgat2 has been shown by others to increase in the absence of Dgat1. Dgat2 knock-out mice are lipopenic and do not survive [158], suggesting that, unlike Dgat1, Dgat2 is more critical in adipose tissue. While it may seem strange that transcripts that encode proteins involved in triglyceride synthesis are up-regulated in rats that do not gain body weight, it should not be surprising since expression of Dgat1 and Dgat2 are influenced by GCs. Most likely, Dgat1 and Dgat2 expression are down-regulated in PRAT due to GR block rather than up-regulated by running. Still, the Dgat data indicates that there is more triglyceride synthesis occurring in RUN rats than in WL+GR BLOCK rats, and RUN rats had higher expression of both Dgats compared to $\mathrm{WL}($ Dgat1 fold change $=1.4662, \mathrm{p}=0.00008$; Dgat2 fold change $=$ $1.4943, p=0.494)$. One possible explanation is that Dgats are up-regulated in RUN rats vs WL+GR BLOCK rats because stored triglyceride is more metabolically active in RUN PRAT and turns over constantly, allowing adipose tissue to readily respond to changes in energy balance [161]. This also supports a second explanation in that the less expression of Dgats in WL+GR BLOCK 
would account for, or be a reflection of, lack of accumulation of TG in adipose tissue. Still, the fact that the RUN rats are not showing a net accumulation of adipose tissue makes the Dgat up-regulation perplexing. The mRNA expression may not reflect a physiological outcome as a $>20$-fold increase in Dgat1 mRNA resulted in only a 2-fold increase in protein expression and function [162].

Prkar2b ( protein kinase, cAMP-dependent, regulatory, type II beta) and Stat5a (signal transducer and activator of transcription 5a) are both highly expressed in all groups' PRAT (PRAT RPKM average $=223.6515$ and 11.8084 , respectively, $n=24)$. Prkar2b is involved in lipolysis and plays a crucial and central role in the regulation of energy expenditure and glucose and lipid metabolism [163, 164] and proliferation of ovarian cells [165], so Prkar2b could contribute to the suspected increased triacylglycerol turnover. Stat5a is activated by GR [166] and shows increased activity during differentiation and adipogenesis [167-169]. KO of either of these transcripts in mice show decreased adiposity [170]. Our interpretation of the findings is that the previous four transcripts (Dgat1, Dgat2, Prkar2b and Stat5a) all seem to go the 'wrong' way given that the RUN rats did not accumulate fat. We suggest as an alternative interpretation, if these four transcripts are thought of as being "lower in WL+GR BLOCK rats," then the 1.5-fold up-regulation in RUN is not surprising. Dgat1, Dgat2, and Stat5a are all influenced by GC and/or GR activity, which would potentially decrease their expression. Also, in WL+GR BLOCK rats, lipid accumulation was attenuated (smaller adipocytes) when compared to RUN. 
Cdc34 (cell division cycle 34 homolog) is highly expressed in all groups' PRAT (PRAT RPKM average $=20.7496, n=24) . \quad$ Cdc34 encodes an ubiquitin conjugating enzyme [171]. Overexpression of this enzyme enhances the ubiquitin proteasome system and promotes degradation of proteins [172]. Notice that ubiquitin $\mathrm{C}(\mathrm{UBC})$ is at the hub of this pathway analysis (Fig. 3.6A: top upregulated pathway in $R U N)$. The ubiquitin-proteasome system controls spatial and temporal cellular processes like cell proliferation and cell death [173]. If this occurs in RUN adipocytes, Cdc34 up-regulation may be enhancing the ubiquination of other transcripts in this pathway shown in Figure 3.6A. Increased transcription would lead to increased mRNA, which would necessitate increased degradation.

Cdc123 (cell division cycle 123) is highly expressed in all groups' PRAT (PRAT RPKM average $=468739, \mathrm{n}=24)$. Cdc123 encodes a protein involved in cell cycle regulation and is regulated by nutrient availability [174].

Overexpression of Cdc123 causes cell cycle delay [174]. Mapk6 (mitogenactivated protein kinase 6, also referred to as Erk3) is highly expressed in PRAT (PRAT RPKM average $=19.1414, n=24)$. Mapk6 is a signaling molecule involved in cell-cycle progression and cell differentiation and protein levels are up-regulated during cell-cycle and terminal differentiation [175]. To sum up, an up-regulation of Cdc123 and Mapk6 in RUN rats may indicate a decrease in cell cycle progression and may help explain a role in the attenuation of total adipocyte number in RUN compared to $\mathrm{WL}$ and BLOCK. 
Taken together, the uniquely up-regulated transcripts in RUN indicate that there is an increase transcripts involved with triglyceride accumulation and an increase in transcripts involved with inhibiting cell cycle progression when compared to $\mathrm{WL}+\mathrm{GR}$ BLOCK. This fits with our findings that $R U N$ rats have fewer PRAT adipocytes despite similar PRAT depot mass.

Transcripts uniquely down-regulated in RUN.

IPA analysis identified "Cell Cycle, Cell Death and Survival, Tumor Morphology" as the top down-regulated pathway, which included 12 of the 28 uniquely down-regulated transcripts for RUN (Fig. 3.6B). Further analysis of these 12 transcripts revealed that 6 of the 12 [cell division cycle 6 homolog (Cdc6), cell division cycle 14 homolog B (Cdc14b), cell division cycle 25 homolog C (Cdc25c), cyclin-dependent kinase 1 (Cdk1), kinesin family member 11 (Kif11), and polo-like kinase 1 (PIk1)] were interconnected in that they were all involved in the same cell cycle regulation pathway as described next. Expression levels (average RPKM for 24 rats) for the 12 transcripts are: Cdk1: 4.4820, Cdc25b: 4.7615, Cdc14b: 2.2736, Kif11: 1.3912, Plk1: 1.1734, Cdc6: 1.5155, Cdk6: 5.6043, Cebpd: 7.8986, Fgf18: 4.2732, Bmp2: 2.7595, Bmp7: 4.2424, and Ccna2: 2.3945.

Cdk1 is a key player in cell cycle regulation [176], and its phosphorylated form (inactive) is increased in presence of dexamethasone [177]. The Cdk1/cyclin B complex is maintained in an inactive, phosphorylated state. The Cdk1/cyclin B complex is activated through dephosphorlyation by $\mathbf{C d c 2 5 b}$, which 
promotes entry into and progression through early mitosis [178]. Interestingly, Cdc25b is activated by GR [179]. Activated Cdk1/cyclin B initiates a positive feedback loop by phosphorylating Cdc25b, thus inactivating it. Cdc14b can also inactivate Cdc25b [180]. Kif11 (Eg5) is involved in the formation of bipolar spindle formation, is essential in human cells, and inhibition results in mitotic arrest and cell death [181, 182]. Cdk1 can phosphorlyate Kif11, which allows for regulation of centrosome separation (8548803). Plk1 is highly expressed in proliferating cells [183] and is one of the proteins that can phosphorylate cyclin [184] as well as initiate a cascade that regulates the accumulation of Kif11 [185]. Cdc6 can be phosphorylated by Cdk1 and Plk1 [186], but Cdc6 can also inhibit Cdk1 and lead to an accelerated exit from mitosis [186]. Note that Cyclin B did not pass the analysis filters but was down-regulated in RUN compared to WL $(R U N \vee$ WL: fold change $=-1.8083, p=0.209)$ and in $R U N$ compared to $W L+G R$ BLOCK (RUN vs. WL+GR BLOCK: fold change $=-2.623, p=0.0442)$. Taken together, it is clear that these six transcripts work together to control, regulate, and maintain cell cycle function. All of these transcripts are down-regulated in RUN rats, which indicate that this particular pathway may not be as robust in the RUN rats as compared to the WL+GR BLOCK rats. This molecular data supports the observed lower total adipocyte number seen in RUN compared to WL+GR BLOCK.

The other six down-regulated RUN transcripts also play a role in cell cycle, proliferation, and differentiation. Cyclin-dependent kinase 6 (Cdk6) was identified as a transcript uniquely down-regulated in RUN, but it was also 
identified as one of the 42 significantly down-regulated transcripts in the entire RNA-seq analysis (Fig. 3.5). Cdk6 combines with Cdk4 to phosphorylating (inactivating) cell cycle inhibitors like pRB [187]. Note that the transcript for pRb (Rb1) is down-regulated in WL+GR BLOCK rats (see discussion below). Downregulation of Cdk6 in RUN would ultimately allow pRb to inhibit cell cycle (decrease the inactivation an inhibitor of cell cycle). Also note that Cdk4 did not pass the analysis filters but had a down-regulated trend in RUN compared to WL (fold change $=-1.3474, p=0.0715$ ) and in RUN compared to WL+GR BLOCK (fold change $=-1.1677, p=0.0672)$. CCAAT/enhancer binding protein $(C / E B P)$, delta. Cebpd is involved in adipocyte differentiation [188, 189], fibroblast growth factor 18 (Fgf18) regulates white AT development and has a regulatory role in adipocyte differentiation and FGF18 has been document in WAT [190], bone morphogenic protein 2 (Bmp2) induces adipogenic differentiation of mesenchymal stem cells [191], and together with bone morphogenic protein 7 (Bmp7), increase lipid accumulation of 3T3-L1 preadipocytes in the absence of the normally required culture media cocktail [192], and cyclin A2 (Ccna2) is expressed briefly before Cyclin A1 in the early cell cycle [193]. The fact that all of these transcripts are down-regulated in RUN rats suggests that cell cycle activity is depressed, and potentially proliferation and differentiation of adipocytes is not occurring as rapidly in RUN rats as in WL+GR BLOCK rats.

Lastly, it is worth mentioning that structural maintenance of chromosomes 2 (Smc2) was identified as unique to RUN, and it was also identified as one of the 42 significantly down-regulated transcripts in the entire RNA-seq analysis 
(Fig. 3.5), but it was not in the top down-regulated IPA pathway. Smc2 is integral to cell cycle progression. Smc2 joins with Smc4 to form a heterodimer and ultimately form condensing, a required protein for sister chromatid cohesion and DNA repair $[194,195]$. While not on the final list, Smc4 was down-regulated in RUN compared to both WL+GR BLOCK $(p=0.0087)$ and WL $(p=0.0078)$ (Table 3.4).

Taken together, the uniquely down-regulated transcripts in RUN indicate that there is a decrease transcripts involved with promoting cell cycle progression, proliferation, and differentiation when compared to WL+GR BLOCK. This fits with our findings that RUN rats have fewer PRAT adipocytes despite similar PRAT depot mass.

\section{Transcripts unique to WL+GR BLOCK.}

Unlike RUN with 8 unique transcripts up-regulated, in the WL+GR BLOCK group there were only two transcripts that were up-regulated and one transcript that was down-regulated (Table 3.4), so an IPA analysis of pathways was not possible. Therefore, the three transcripts that are unique to BLOCK will be discussed individually. Transcripts uniquely up-regulated in WL+GR BLOCK.

The transcripts for angiopoietin-like 4 (Angpt|4) and cell division cycle 73 (Cdc73) were filtered out of 173 genes as the only two transcripts up-regulated uniquely to WL+GR BLOCK. 
Angpt14 was highly expressed in PRAT (average RPKM $=157.9973$, $n=24$ ), was expressed at higher levels in $W L+G R$ BLOCK compared to $W L$ (fold change $=1.775, p=0.0023$ ), and was also expressed at higher levels in $W L+G R$ BLOCK than RUN (fold change $=1.742, p=0.0499$ ). ANGPTL4, one of seven secreted ANGPTL proteins, is an adipokine that is predominately expressed in adipose tissue and liver and is involved in lipid metabolism [196] and triacylglycerol homeostasis [197]. It was originally identified as fasting-induced factor from liver [198] and is a downstream target of PPAR $\alpha$ and PPAR $\gamma$ during pre-adipocyte differentiation [199]. Angpt/4 has been linked to circulating lipids and high density lipoprotein and triglyceride concentrations [200-202] and as a lipoprotein lipase (LPL) inhibitor [203, 204]. Since Angpl4 is up-regulated in WL+GR BLOCK rats, LPL may be inhibited, which would limit the fat entry into adipocytes, thus attenuating fat accumulation. The Lpl transcript was more highly expressed in RUN than in WL+GR BLOCK ([RUN versus WL+GR BLOCK] fold change $=1.446, p=0.0003$ ), indicating that the Angpt|4 up-regulation in WL+GR BLOCK may be influencing LPL action in these rats.

Angpt|4 expression is induced during adipocyte differentiation and levels continue to rise once the adipocyte is fully differentiated [205-207], supporting the observation of increased total adipocyte number in WL+GR BLOCK rats.

ANGPTL4 stimulates the increased activation of c-Jun and Junb both of which are transcription factors involved in proliferation [208]. Junb was expressed at higher levels in WL+GR BLOCK than RUN (RUN versus WL+GR BLOCK fold change $=-1.352, p=0.0422$ ), suggesting that a downstream target of Angpt/4 
that is involved in cell proliferation is increased in WL+GR BLOCK rats, providing further molecular support of increased total adipocyte number in WL+GR BLOCK rats.

Our finding that Angpt/4 expression is higher with GR block is puzzling since GR is a transcription factor for Angptl4 [209]. In a fasting-induced Angptl4 model, GR blockade by RU486 prevented increased Angptl4 expression in liver white epididymal adipose tissue in mice [209], but this appears to be limited to fasting-induced models [197] as the GR blockade did not prevent an change in Angptl4 mRNA levels when the animals were fed ad libitum, only when they were fasted. There are other transcription factors for Angptl4 including PPAR $\gamma$ [205, 207], and PPAR $\gamma$ has been shown to reverse effects of GC inhibition [210]. In the WL+GR BLOCK animals, the Pparg transcript was highly expressed (RPKM $=79.377)$, suggesting that PPAR $\gamma$ may contribute to the Angpt/4 up-regulation.

Cdc73 (Cell division cycle 73) is a medium-expressed transcript (average $\mathrm{RPKM}=9.2473, \mathrm{n}=24)$, was expressed at higher levels in $\mathrm{WL}+\mathrm{GR}$ BLOCK compared to $\mathrm{WL}$ (fold change $=1.190, \mathrm{p}=0.0061$ ), and was also expressed at higher levels in WL+GR BLOCK than RUN (fold change $=1.301, p=0.0092$ Cdc73 one of several members of the polymerase II-associated factor (PaF) complex. No data was found on $\mathrm{Cdc73}$ and adipose tissue, but it plays a role in cell proliferation [211] and is involved transcription initiation and elongation and mRNA processing [212]. Cdc73 negatively regulates $b$-catenin, Cyclin D1 and cMYC [211], thus acting to inhibit cell cycle, which supports our findings that RUN rats have fewer PRAT adipocytes despite similar PRAT depot mass. 
Transcript uniquely down-regulated in WL+GR BLOCK.

The retinoblastoma $(\mathbf{R b} \mathbf{1})$ transcript was filtered out of 173 genes as the only gene down-regulated uniquely to $\mathrm{WL}+\mathrm{GR}$ BLOCK. Rb1 is a mediumexpressed transcript (RPKM average $=1.7749, \mathrm{n}=24$ rats) and was expressed at lower levels in $\mathrm{WL}+\mathrm{GR}$ BLOCK compared to $\mathrm{WL}$ (fold change $=-1.128, \mathrm{p}=0.041$ ) and was also expressed at lower levels in WL+GR BLOCK than RUN (fold change $=-1.163, p=0.0132$.

$\mathrm{Rb} 1$ transcribes the tumor suppressor protein $\mathrm{pRb}$ protein and, when dephosphorylated, acts as a negative regulator of the cell cycle. Interestingly, GC have been shown to work through Rb1 dephosphorylation to induce $\mathrm{G} 1$ cell cycle arrest [213]. Rb1 plays a role in tumor suppression, cell cycle progression, apoptosis, and cell differentiation [214-217], and in adipocytes, Rb1 facilitates preadipocyte differentiation into adipocytes [218]. Rb1 is lowly expressed $(R P K M=1.579)$ in PRAT of $W L+G R$ BLOCK rats, but the down-regulation indicates that this 'brake' on cell cycle is decreased, thus promoting cell cycle progression and differentiation.

The three transcripts discussed above that are unique to $\mathrm{WL}+\mathrm{GR}$ BLOCK provide molecular evidence that transcripts promoting cell cycle progression, cell proliferation, and differentiation are present at a higher level in WL+GR BLOCK compared to RUN, and a transcript that inhibits cell cycle progression is present at lower levels in WL+GR BLOCK compared to RUN. Taken together, this data 
supports the observed perirenal adipose phenotype of increased total adipocyte number in WL+GR BLOCK rats.

The unique transcripts that were different between RUN and WL+GR BLOCK were investigated because our findings indicated that RUN and WL+GR BLOCK used different pathways to attenuate visceral adipose expansion. However, given that some of the transcripts were common to RUN and WL+GR BLOCK, there is some molecular similarity between the two groups.

\section{Confirmation of conclusion from Chapter 2}

RNA-seq analysis of cyclin A1 showed that it was down-regulated in RUN rats (fold change $=-1.7229, p=0.0016$ ), which confirmed mRNA analysis from Chapter 2 that showed Cyclin A1 was down-regulated in RUN rats (fold change $=$ $-1.5549, p=0.0198)$. Further analysis showed that $41.0 \%$ of the 173 hypothesisspecific, cell cycle, proliferation, and differentiation transcripts were different between RUN and WL. A brief review of the 18 transcripts that were upregulated in RUN include those involved with triglyceride accumulation and inhibition of cell cycle progression, much like when RUN was compared to WL+GR BLOCK. Many of the 53 transcripts that were down-regulated in RUN are involved in cell cycle progression, proliferation, and differentiation. This confirmed that there are many DETs between RUN and WL in addition to cyclin A1, thus adding to the molecular evidence from Chapter 2 and allowing us to hypothesize that a sudden decrease in activity causes an increase in adiposity 
due to increases incell cycle progression, proliferation, and differentiation of preadipocytes.

\section{Limitations of the study}

A limitation of study was that there was not a RUN+GR BLOCK group. Without this group, we cannot fully determine the effects of RU486 in conjunction with activity (RUN+BR BLOCK). In other words, would have RU486 negated the effects of RUN, and caused an increase in transcripts involved with cell cycle progression, proliferation, and differentiation as was observed in the WL+GR BLOCK group? Future studies can be designed to address these hypotheses and limitations. In defense of not having a RUN+GR BLOCK group, the way the hypothesis was stated looked at 1) how does RUN attenuate PRAT gains seen with WL? 2) how does WL+GR BLOCK attenuate PRAT gains seen with WL? and 3) does RUN and WL+GR BLOCK do this the same way? In this regard, a RUN+GR BLOCK was not necessary.

Another limitation is that we did not block GR specifically in PRAT. Instead we had a systemic GR block. This calls into question the effect that other GC target tissues such as liver and skeletal muscle and kidney had on adipose tissue. Given the interconnectedness, it would be unlikely that the effect we saw in PRAT is limited to adipose physiology. It is more likely that it was the interplay of altered liver, skeletal muscle, and kidney function that contributed to 
the effect we saw in PRAT. To address this, an osmotic pump or other device to locally administer a drug could be used to isolate GR block specifically to PRAT.

In conclusion, we found that RUN and WL+GC BLOCK did NOT affect cell cycle, proliferation, and differentiation in the same manner. We then identified which transcripts differed between RUN and WL+GR BLOCK to identify potential key transcripts and pathways unique to RUN and to $W L+G R B L O C K$ to generate new hypotheses. One such hypothesis is that there is increased triacylglyceride turnover in RUN rats, and that BLOCK and WL attenuates this turnover. This hypothesis is based on the up-regulation of Dgat1, Dgat2, and Stat5a combined with the lower PRAT total adipocyte number in RUN. Another hypothesis is that there is decreased lipid metabolism in RUN and WL+BR BLOCK rats compared to $\mathrm{WL}$ rats. This is based on whole-transcriptome analysis and the top upregulated pathways for both $[R U N$ vs. WL] and [WL+GR BLOCK vs. WL] involve carbohydrate and lipid metabolism. We also extended the findings from Chapter 2 and found that indeed, PRAT from WL animals show increased levels of transcripts involved in in cell cycle progression, proliferation, and differentiation genes compared to RUN. Finally, data is presented in this study that can help manifest future hypotheses that help further understand how adipose tissue responds to a sudden decrease in activity. 


\section{CHAPTER 4}

\section{Discussion}

I hope to convey my thought process in developing the hypotheses, designing the experiments, and interpreting the results throughout this discussion chapter. I will present my discussion in chronological order and try to be clear when a new insight or bit of data was discovered that either altered or shaped the way I approached the experiments.

\section{Formulation and testing of hypotheses}

As described in detail in Chapter 1, I formulated my hypothesis based on four major questions:

1) Does the way in which adipose tissue expands depend on the age of wheel lock?

2) How does adipose tissue expand with 7 days of wheel lock?

3) Is this expansion healthy or unhealthy?

4) Do glucocorticoids play a role in this adipose tissue expansion?

My overall hypothesis was that seven days of wheel lock in49-56 day old rats and 70-77 day old rats would result in gains in visceral adipose mass seen in age-matched sedentary rats concurrent with an increase in adipocyte size and inflammatory mRNA expression. Further, glucocorticoid block would attenuate the visceral adipose depot gains seen in wheel lock $(\mathrm{WL})$ in 70-77 day old rats. 
In order to test these two hypotheses, I needed to implement our WL model in a very systematic and organized fashion. Rats were randomly selected from an ongoing rat breeding study in the Booth lab where we are breeding rats who are, and who are not, "motivated" to run in a voluntary running wheel. When rats were available, I received anywhere from 2 to 5 male, Wistar rats who were "likely to be motivated to run." I performed the experiment on the 49-56 day old rats first, then, once I had enough rats (statistical power tests indicated that I needed at least 6 rats in each group), I moved on to the 70-77 day old rat experiment. From my conversations with Professor Venkataseshu Ganjam, I learned that blocking glucocorticoids also prevented enlargement of visceral adipose tissue, much like running. So I decided to compare both running and glucocorticoid block at the molecular level to test the hypothesis that their molecular mechanisms would be the same. I assigned rats to the glucocorticoid receptor (GR) block experiment at the same time as I was collecting data on the 70-77 day old rats. I did this in order to be efficient with rats, running wheels, and time. When possible, I assigned rats from the same family to each experimental group. For example, if I received five rats at one time, I would assign one rat to $R U N$, one to $W L$, one to SED, one to $W L+G R$ BLOCK and one to $W L+V E H I C L E$. There is a great deal of inter-family variability in terms of voluntary running distances and body weight. While there was no way to predict the rats running at the time of assigning groups, I maintained a random method of assigning the rats (in short, I took a sheet of paper with the rats ID numbers, put them in a can, and drew them randomly). The hope was to distribute the over- and under-achieving 
rats with regard to voluntary wheel running equally among the groups. Statistical analysis of the data indicates that this worked well (groups of equal voluntary physical activity, i.e. groups that were running and groups that were sedentary, showed no differences in body weight, body fat, and distance run).

With this model in place, I tested the overall hypothesis in two experiments, each with their own hypothesis. These were presented in Chapter 2 and Chapter 3 and will be discussed in the order I performed experiment and analyzed the data. I will first discuss my rationale for interpreting my data in the context of childhood obesity, then I will discuss the first experiment I performed (presented in Chapter 2) followed by the second experiment I performed (presented in Chapter 3).

\section{Rationale for interpreting WL data in the context of childhood obesity}

My approach to interpret data from Chapters 2 and 3 is to put it into the context of human childhood growth and childhood obesity. In this context, and in my opinion, these two studies collectively represent one of the most in-depth investigations into adipose tissue expansion in a model of childhood obesity. To my knowledge, no one has interpreted a wheel lock or "cessation of physical activity" rodent model in the context of childhood growth and catch-up fat, making the findings in my dissertation novel and insightful. Further, no one has published adipose tissue phenotype or transcriptome at two separate time points in young, growing animals. There is much more that can be done (see Chapter 
5), but the studies presented in this dissertation provide new insights into adipose tissue expansion in a young, growing animal.

The rationale for framing my dissertation work around the childhood obesity paradigm is in large part due to the crisis facing the U.S. health system led by poor lifestyle choices. Having worked with adult humans, I realized that, anecdotally, it is difficult to change adult behavior. Scientific studies support my observations. An American Dietetics Association poll reported that $40 \%$ of overweight people said they did not want to give up their unhealthy lifestyles in order to be healthy [219]. Adults often do not adhere to a diet and exercise program aimed at improving their health [220, 221], yet people turn to a pill or supplement for help losing weight, often with no noticeable benefit [222] due to, in my opinion, poor compliance or lack of potency of the supplement or pill. Also, having worked with children and teenagers as public school teacher and coach, I realized that, anecdotally, it is easy to get children to be physically active.

Providing them with the opportunity and environment to stay physically active promotes a desire to remain physically active throughout adolescences and adulthood, which seems to keep them healthier, more likely to attend classes and activities, and more likely to perform better in school. Again scientific studies support my observation. Tracking studies, while difficult to perform, show that physically active children tend to become active adults [223]. There is overwhelming evidence that physically active children are sick less and have better school attendance [224]. Further, multiple studies provide evidence that physically active children have better cognitive function and learning skills [225, 
226]. Still, there is clearly more work to be done concerning lifestyle and health in children. Currently, $18 \%$ of children between the ages of $6-19$ are classified as obese [4]. This occurs concurrently with a dramatic decrease in physical activity. Troiano et al. [227] measured the physical activity levels of U.S. children by accelerometer and found that $42.0 \%$ of children between the ages of $6-11$ years attain sufficient physical activity by the U.S. guidelines (60 minutes of moderateto-vigorous activity daily), but in contrast, only $8.0 \%$ of children between the ages of $12-15$ years attain sufficient physical activity. By the time these children graduate from high school, $92.4 \%$ of them are not participating in adequate daily physical activity. This sets them on a trajectory for life-long struggles with weight, obesity, and metabolic complications, as well as increased risk for depressivelike disorders [228].

Taken together, there is clearly a need to understand the etiology of obesity and mechanisms by which children develop obesity and lifestyle choices that set them up to be more- or less-likely to develop obesity. My two studies provide unique insight into this paradigm and will be discussed next.

\section{The story from my initial roadmap for Chapter 2, roadblocks, unexpected findings, and alternative routes taken to complets my journey to the completion of the manuscript in Chapter 2.}

In my first study, I tested the hypothesis that 7 days of wheel lock would 1) increase the rate of percent body fat growth and adipose depot masses to levels seen in age-matched SED rats in both ages of rats; 2) result in a visceral adipose 
depot expansion via hypertrophy in rats 49-56 days of age and via hyperplasia in rats 70-77 days of age, and 3) increase inflammatory mRNAs in adipose tissue before other selected categories of mRNAs in rats 49-56 days of age but not 7077 days of age. These hypotheses were based on preliminary data from the Booth lab (described in Chapter 1 and Chapter 2), i.e. my literature review on adipose expansion and inflammation, and were driven by three of my four major questions: 1) Does the way in which adipose tissue expands depend on the age of WL? 2) How does adipose tissue expand with one week of WL? 3) Is this expansion healthy or unhealthy?

At the onset, I posited that WL would evoke significant alterations in proinflammatory genes. Even though I was primarily looking at inflammation, it is clear from the hypotheses that I was also interested in the question of hypertrophy versus hyperplasia. To get a broader snapshot of what was going on in adipose tissue with WL, I decided to measure other transcripts that represented functional gene categories (pro-inflammatory, hypoxic, adipokine, and adipogenic). I hypothesized that I would see an increase in inflammation with WL, but I didn't want to rule out other interesting things (like proliferation and differentiation transcripts). What ended up being the most interesting finding was that WL initiates adipocyte hyperplasia in epididymal adipose tissue (EAT) and perirenal adipose tissue (PRAT) of 70-77 day old rats. This was unexpected, but fits nicely into the context of the childhood obesity public health message as I will describe in the next few pages. 
Other studies using a "decrease in physical activity" model in young rats.

Very few studies have been performed on the effect of voluntary physical activity with cessation of physical activity in young animals, specifically in the context of visceral adipose tissue expansion and total adipocyte number. Data from this dissertation provides additional insight, explanation, and possibly resolution into results from previous experiments of this nature. The studies addressing cessation of activity and its effect on adipose tissue are discussed next.

Hatano et al. [229] investigated treadmill exercise training on male, Wistar rats age 28-91 days of age. Hatano found that there were fewer total epididymal adipocytes in the treadmill trained rats than in sedentary rats. My data from Chapter 2 provides a possible explanation to this; the reason this is occurring is that physical activity or exercise suppresses cell cycle, proliferation, and differentiation in adipose tissue.

Schroeder et al. [230] provided male OLETF rats, an animal model of obesity characterized by hyperphagia-induced obesity, and LETO rats, used as a control for the OLETF, access to a voluntary running wheel from 22-45 days of age, after which time the wheel was locked until sacrifice at 90 days of age. Schroeder found no difference in inguinal adipocyte number between LETO rats that had access to the running wheels and those that did not, but a higher number of adipocytes in OLETF rats that also had access to running wheels. The obvious differences between this study and my studies are the time of wheel 
lock and the duration of wheel lock. Schroeder locked the wheel at a time that I found the adipose tissue to be expanding by hypertrophy and hyperplasia (see Chapter 2, Figure 8.) In addition, the OLETF rat is a model of obesity, while the wild-type Wistar is not. The data from Chapter 2 provides a probable explanation as to why Schroeder did not see a difference in adipocyte number; the age of WL occurred during an age where we hypothesize there is little to no WL effect on adipocyte size or VAT total adipocyte number (Chapter 2, Figure 11).

Gollisch et al. [231] provided young Sprague-Dawley rats access to voluntary running wheels for four weeks. Gollisch found that voluntary running produced smaller subcutaneous and visceral adipocytes than the sedentary control rats, but did not alter cell number in either depot. These results agree with previous data from the Booth laboratory [61] as well as data presented in Chapter 2 and can be explained by the age of sacrifice. These rats were also sacrificed during the 49-56 day old age, where I found that wheel lock offers little to no effect on adipocyte size or VAT total adipocyte number (Chapter 2, Figure 11).

Finally, our results resolve previous data discrepancies in the Booth laboratory between two WL studies $[60,61]$ with conflicting results (see introduction for details). The previous two studies were conducted with Fischer 344 x Brown Norway (FBN) rats, while my studies were conducted with Wistar rats. This rat strain difference aside, I concluded that there was not a discrepancy between these two studies, rather both were correct, but the age of growth when WL takes place affects whether increase in adipose tissue mass 
employs increased hypertrophy (49 day old rats) or hyperplasia (70 day old rats). We found that adipocyte diameter increased from 49 to 56 days in both RUN and SED groups, indicating that at this age rats are still expanding adipocyte mass by hypertrophy. While we can say we did not observe a WL effect, we did observe adipose expansion via hypertrophy. However, in the 70 to 77 day old rats, there was a clear cessation of hypertrophic growth, and WL rats exhibited hyperplasia. Despite this resolution, a number of potential explanations should be discussed in regards to these two rat strains. Both our current study (Wistar rats) and our previous study (FBN rats) [60] used male rats of similar ages and similar duration of wheel lock ( 7 days of WL from 63-70 days of age for FBN rats and 70-77 days of age for Wistar rats). However numerous qualitative differences occurred between the two strains. For the age range during $\mathrm{WL}$, body masses were greater in Wistar (351.5 - 417.5 g) vs. FBN (207.2 - $233.8 \mathrm{~g})$ and, compared with previous studies, body fat attributes were higher in Wistars in the current study. Specifically, \% body fat was $8-13 \%$ for Wistars vs. $4.1-6.8 \%$ for FBN and epididymal fat mass was 3-6g for Wistar and 1.2-1.6 $\mathrm{g}$ for FBN [60]. These strain differences also mirror Levin's data [120]. In a third strain (Sprague-Dawley), Levin et al. found that only 3 wks of voluntary wheel running sufficed to prevent increases in adiposity for 10 wks after wheel removal. Hence, the three strain differences may explain divergent findings, which, while may be viewed as a limitation to some, may actually reflect the large intra-individual differences in susceptibility for fat gain in the human population. For example, during overfeeding of 12 pairs of young, adult, male monozygotic twins, individual 
changes in body composition and topography of fat deposition varied considerably with a range of $4.3-13.3 \mathrm{~kg}$ [232]. Thus, heterogeneity of fat gain characteristics exists in both humans and rats.

My findings placed in the context of early-life adipocyte number set point.

As stated above, one major, yet underappreciated, reason that findings from this study are highly relevant to the current childhood inactivity and obesity public health issue is that puberty is a critical time of adipose tissue expansion [5, 233], where many believe the number of adipocytes is set in humans for adulthood [6]. The first study, to my knowledge, to look at total adipocyte number in adolescent (10-18 years of age) was published by Chumlea et al. in 1982 [234]. Chumlea reported that increases in body fat during adolescents were likely due to increased adipocyte number. Around this time, Roche published in 1981 the adipocyte-number hypothesis [51], which stated that the number of adipocytes is fixed in early life and this number predestines a person to be lean or obese depending on the number of adipocytes a person is 'set' at. In 1991, Cartwright discussed the switch from adipocyte hyperplasia to hypertrophy at 12-14 weeks of age in chickens [50]. Cartwright suggested that adipocyte number is more related to body mass development, while adipocyte size is more related to body composition [50]. Spalding et al. [6] pioneered work in total adipocyte number and turnover that was published in Nature in June of 2008. Using ${ }^{14} \mathrm{C}$ incorporation into genomic DNA from nuclear bomb tests in the late 1950s and early 1960s, Spalding was able to estimate the 'birth date' of a cell and retrospectively find the age of adipocytes in humans. Spalding concluded 
that the half-life of the average adipocyte to be $\sim 8.3$ years and approximately $10 \%$ of fat cells renew annually in all adult ages and at all levels of body mass index. She further suggests that this number is tightly regulated and dying adipocytes are replaced by new adipocytes to maintain an overall consistent number of total body adipocytes.

However, not everyone agrees with Spalding and her adipocyte set-point hypothesis. Sun and Scherer [43] propose the following questions: 1) Given that obesity is associated with higher adipocyte turnover due to accelerated adipocyte death, might there be situations where adipocyte recruitment (hyperplasia) is greater than adipocyte death and we see a net increase in total adipocytes? 2) What is the mechanism by which a new adipocyte is recruited to replace a dying one? These are excellent questions that we do not yet have an answer to. Part of Scherer's skepticism may be due to a study he published in 2007 [54]. Scherer's group developed an ob/ob mouse obesity model (ob/ob mice do not produce leptin) that overexpressed adiponectin. The result was a very obese mouse with a "healthy," phenotype (normal triglyceride levels, normal visceral adipose tissue mass, and normal insulin sensitivity) with an increased number of small adipocytes and reduced macrophage infiltration. This occurred concurrently with a partitioning of fat away from the viscera such that these ob/ob-adiponectin overexpressing mice had increases in subcutaneous adipose depots and a visceral depot phenotype that matched wild type animals. My view is that while this is proof that adiponectin is a central player in altering the metabolic homeostasis in adipose tissue, and proof that hyperplasia can occur in older 
rodents, the condition of the mice in this study is a unique genetic condition in which adipose tissue in a genetically, obesity-prone animal is induced by another genetic modification and thus makes it difficult to translate into a real-life situation. My position as of now is to adopt the working hypothesis that we have a relatively stable number of adipocytes that is set after puberty. I base this hypothesis on human [6] and rat [10] literature. I propose studies to test this hypothesis in Chapter 5.

Whether or not the total adipocyte number is set at early adulthood, it is still believed that the more adipocytes a person has, especially visceral adipocytes, the more susceptible they become to obesity later in life [7]. If we believe that 1) the number of adipocytes is set early in life and remains relatively stable throughout the remainder of life and 2) the more adipocytes you have, the more likely you are to become obese it seems to be, at least theoretically, advantageous for health to set the total number of visceral adipocytes low. I found that the total adipocyte number in the epididymal and perirenal adipose depots remains lower in physically active rats compared to sedentary rats and rats that underwent one week of wheel lock (Chapter 2, Figure 8). This, in and of itself, is a novel finding that prompts me to hypothesize that if we keep kids physically active through puberty and into early adulthood, they will be less likely to become obese later in life. This paragraph is pure speculation, and I will further address my hypothesis in Chapter 5.

To place my story in its true timeline, it was not until writing this dissertation did I begin to integrate my observations in the manuscripts in 
Chapters 2 and 3 . Therefore following is an overview of my current thinking. As we advance our knowledge about adipose tissue expansion, we will continue to evolve in our thinking. The predominant belief currently is that adipose tissue growth after sexual maturity, as seen in adult animals, occurs via hypertrophy [6], and only in extreme cases of increased adipose tissue expansion does hyperplasia take place [235]. However, location of adipose tissue depots is critical as Singh et al. [236] reported that, while abdominal adipose expansion occurs via hypertrophy, lower body, subcutaneous expansion occurs via hyperplasia. Clearly we are still learning about adipose tissue expansion, but in the context of a young, growing animal, my results from Chapters 2 and 3 provide novel insight: in 49-56 day old rats rats, a sudden decrease in physical activity shows little to no disturbance in visceral adipocyte phenotype or mRNA expression and a "progress as usual" environment. However, this same decrease in physical activity in 70-77 day old rats shows an altered increase in visceral total adipocyte number and adipocyte mRNA expression towards increased cell cycle, proliferation and differentiation.

My interpretation of this data is that expansion via hyperplasia in the 49-56 day old rats was not observed, not because the rats are failing to produce new, mature adipocytes, but rather because rats are producing new, mature adipocytes during a rapid growth phase. This is where age-matched groups are valuable. The way I identified that the WL group underwent hyperplasia was to compare it to the age-matched RUN group. In the 49-56 day old groups, epididymal total adipocyte number was equal between $56 d$ RUN and $56 d 7 d-W L$, 
thus I concluded that hyperplasia did not take place. However, I also observed an increase in epididymal total adipocyte number between 49d RUN and 56d RUN. I interpret this to mean that these 49-56 day old rats rats are still undergoing hyperplasic adipose expansion even while maintaining daily physical activity. Had I not included the age-matched 56 RUN group, it would have been tempting to deduce that hyperplasia was occurring in the 49-56 day old rats since $49 d$ $R U N<56 d 7 d W L$. This is clearly not the case. I conclude that $49-56$ day old rats were going to increase epididymal cell number at a similar rate whether they were physically active or wheel locked. Now, the 70-77 day old rat data tell a different story. There was no difference between 70d RUN and 77d RUN total adipocyte number or in 70d SED and 77d SED total adipocyte number in epididymal or perirenal depots. My interpretation of this data is that at this age, these rats are no longer undergoing hyperplasic adipose expansion unless there is a drastic metabolic disturbance, like wheel lock (sudden decrease in physical activity). What is still unknown is if wheel lock is performed at adulthood, would we see an increase in total adipocyte number in these depots. This question is addressed in Chapter 5.

"Healthy" obesity, characterized by smaller adipocytes and absence of an inflammatory component, is not the problem.

I want to be clear that obesity in and of itself is not necessarily the problem; rather it is the type of adipose tissue expansion that occurs during obesity (hypertrophy versus hyperplasia). Some forms of obesity actually have a 
'healthy' phenotype [235]. Healthy obesity has been characterized by the absence of any metabolic disorder in an obese individual $\left(\mathrm{BMI}>30 \mathrm{~kg} / \mathrm{m}^{2}\right)$. In addition, adipose tissue of healthy, obese people is characterized by small adipocytes [52] and minimal fat in the viscera [237, 238]. So, in this context, my study provides valuable insight. I found a way to lower what I define as the setpoint of total adipocytes (stay physically active). I also found that if you decrease activity at sexual maturity, you see an increase in visceral total adipocyte number. The health effects of this are unknown, but based on current literature $[23,26-34,70]$, an increase in visceral total adipocyte number is not a favorable phenotype for long-term risk for obesity based on adipocyte hypertrophy and the fact that more adipocytes is associated with higher risk for developing obesity later in life [7]. In my studies, rats that maintained physical activity had 'healthier' visceral adipocyte phenotypes in terms of body fat \%, visceral adipocyte size, and visceral total adipocyte number.

I was obviously surprised that I did not see an increase in any inflammatory markers, given that there are numerous studies that point to increased adiposity is accompanied by an increase in inflammation [19, 239241]. It is clear that physical inactivity leads to accumulation of visceral fat and eventually the activation of inflammatory pathways, but in our model, we failed to see this. Studies point to adipose tissue remodeling and extracellular matrix components such as collagen as important variables in adipocyte differentiation and contributors of adipose tissue inflammation during adipose tissue expansion [242-246], but many of these studies were performed in culture, in chronically 
overweight or obese subjects, or in animal models of high-fat diet induced obesity. Other studies point to hypoxia as a trigger for inflammation in adipose tissue expansion and remodeling [104-106]. HIF-1 $\alpha$ functions as a master regulator of cellular and systemic homeostatic repose to hypoxia by activation transcript of many genes, including those involved in energy metabolism, angiogenesis, apoptosis, and other genes whose protein products increase oxygen delivery or facilitate metabolic adaptation to hypoxia (from genecards.org). I measured mRNA expression of HIF-1 $\alpha$, an upstream indicator for adipose tissue dysfunction [106]. HIF-1 $\alpha$ mRNA expression in WL showed no difference compared to RUN or SED animals, suggesting that this pathway is not present in our model. One week of WL may be too brief to initiate the inflammatory pathway. Perhaps if we allowed the rats to undergo WL for weeksto-months, we would see an increase in visceral adipose inflammatory markers compared to physically active rats. This point is discussed in Chapter 5 . The important finding from the mRNA data was that a proliferation gene was upregulated in EAT and PRAT of 70-77 day old rats without any changes in inflammatory mRNAs.

\section{Chapter 3: Glucocorticoid block attenuates fat gains by a different mechanism than physical activity.}

While the thought of looking at cell cycle, proliferation, and differentiation transcripts was not specifically stated in my overall hypothesis, the findings from 
Chapter 2 made it clear to me that the "visceral expansion via hyperplasia" story needed to be followed up. So, the primary purpose of Chapter 3 was to examine the role of glucocorticoids on the adipose gains during decreased activity, but a secondary purpose was added to test the hypothesis presented at the end of Chapter 2 that visceral adipose tissue, specifically EAT and PRAT, is expanding by hyperplasia in this rat model due to an increase in cell cycle, proliferation, and differentiation induced by WL.

In this study, I tested the hypothesis that compared to WL rats, rats that had access to voluntary running wheels and rats that underwent WL plus a glucocorticoid receptor block would show similar changes as WL in PRAT transcript expression involved in cell cycle, proliferation, and differentiation. The challenge, and dare I say "goal," of the study in Chapter 3 was to determine whether I could administer a drug at the same time as WL that had the same suppressive effects on adipose tissue gain as did running. This concept goes against my philosophy as an exercise physiologist, and against the philosophy of the Booth Laboratory, in that our goal for humans is that they incorporate physical activity into their lifestyle and avoid taking a drug, which, at best, could only partly mimic physical activity. But, in the instances of forced physical inactivity, like injury, the idea of taking a drug temporarily to reduce fat gains is appealing, practical, and ethical. In addition, this concept is scientifically justified: if we could show that blocking glucocorticoid action had the same effects in a visceral adipose depot as maintaining physical activity (wheel running), then we could 1) conclude that running interacts with the glucocorticoid system as a 
means to attenuate fat accumulation and 2) suggest that RU486 administration reproduces the PRAT phenotype seen with physically active and is an appropriate adipose-maintenance drug in times of decreased physical activity.

I was not the first person to think about reducing GC action as a 'fat loss' solution. Reducing active glucocorticoids (cortisol in humans and corticosterone in rodents) is an effective way to combat obesity and metabolic syndrome [247] and to treat individuals with excess glucocorticoid levels like Cushing's syndrome [132]. In addition, supplement companies are capitalizing on the concept that reduced glucocorticoid (GC) action can help fat loss. Supplement companies have taken advantage of this concept and produced products such as CortiSlim $^{\mathrm{TM}}$ that putatively reduce cortisol production. Taken together, blocking GC action in a situation where we know we will see an increase in adiposity seemed like a potential part of an exercise mimetic.

Of course, this was not what I found. Physical activity may influence glucocorticoid action in the suppression of adipose gains, but physical activity mechanistically works through a different pathway than glucocorticoids. I also found that RU486 is not an appropriate "physical activity mimetic" because it not only suppressed adipose tissue gains with decreased physical activity; it suppressed lean body mass gains too, which is not a desired health outcome, especially in a growing animal. This is an important finding, especially in the context of our over-supplemented and over-medicated population: while RU486 attenuated gains in adipose tissue, it also attenuated gains in lean tissue mass in a pre-adult animal model. While the physiology behind these effects were 
beyond the realm of the current study, it is worth mentioning, contrary to my findings, excessive GCs leads to decreased protein synthesis and increased proteolysis [147] and in the presence of excessive GCs, RU486 prevents muscle atrophy [148]. I did not see elevated corticosterone levels in my rats and all rats were gaining lean body mass, so the lean body mass attenuation I observed was an unexpected finding.

\section{Rationale for choosing a glucocorticoid blocker}

The rationale behind choosing a glucocorticoid blocker as the 'fat attenuation' drug in Chapter 3 was this: 1) a literature search on GCs and their effect on adipose tissue (see introduction for details), and 2) I had been studying the enzyme 11-beta hydroxysteroid dehydrogenase type 1 (11ßHSD-1) with Professor Venkataseshu Ganjam prior to formulating this experiment. I felt it is worth discussing this enzyme here because of its powerful role in GC action, and because I spent about one year of working on this enzyme in another experiment.

My data on the effects of exercise training on $11 \beta \mathrm{HSD}-1$ in pigs is found in Appendix C. $11 \beta \mathrm{HSD}-1$ is a bidirectional enzyme that can activate and inactivate GCs, but in most tissues, the reductase activity predominates, thus generating active cortisol from inactive cortisone (active corticosterone form inactive 11-dehydrocortisone in rodents) [126]. $11 \beta \mathrm{HSD}-1$ is abundantly expressed in adipose tissue and is suggested to be a major etiological factor in the development of visceral obesity and the metabolic syndrome [126, 152-155]. 
As of late, $11 \beta \mathrm{HSD}-1$ has been targeted as a key enzyme in the local activation of GCs in adipose tissue and is currently the focus of some pharmaceutical companies as a therapy to lower visceral adipose tissue mass [149].

The link between glucocorticoids, $11 \beta \mathrm{HSD}-1$, and physical activity is that voluntary physical activity in a young, growing animal appears to up-regulate $11 \beta \mathrm{HSD}-1$ and be protective against metabolic alterations and obesity [248, 249]. Coutinho et al. [250] found that hamsters that were exercise training had 2.7-fold higher $11 \beta \mathrm{HSD}-1$ enzyme activity in visceral adipose tissue compared to sedentary hamsters concurrent with less visceral adipose tissue mass. Campbell et al. [73] reported that exercise training increased $11 \beta \mathrm{HSD}-1$ protein in both subcutaneous and perirenal adipose tissue in hamsters and concluded that GCs were a significant contributor in the adipose tissue phenotype change and decreased adipose tissue mass that occurs during exercise training. Recently, Rajia et al. [249] reported that in a model of rodent pup-obesity induced by maternal obesity, rats that had access to voluntary running wheels post weaning with ad libitum access to a chow diet reduced hypothalamic 11ßHSD -1 mRNA expression and reversed the deleterious metabolic effects of maternal obesity. Now, I re-interpret the literature that the metabolic effects may be due to physical activity, rather than $11 \beta \mathrm{HSD}-1$. However, I interpret that when taken together, it seems that physical activity increases $11 \beta \mathrm{HSD}-1$ expression in adipose tissue and leads to a favorable phenotype. One obvious question that arises is, what happens during a sudden decrease in inactivity? Does this heightened sensitivity to GCs that occurs with physical activity and exercise training (discussed in the 
introduction) along with increased expression of $11 \beta \mathrm{HSD}-1$ causing deleterious effects? It was along this line of thinking that I chose to block the glucocorticoid receptor during WL.

Other studies incorporating glucocorticoid block in young rats.

There are few studies that investigate glucocorticoid block in the context of young animals and adiposity, and no study, to my knowledge, that looks at glucocorticoids in the context of decreased physical activity. Langley and York [88] found that 15 days of RU486 administration in young 5-week old Zucker rats stopped fat deposition in the Zucker rats and increased protein deposition to levels seen in lean-vehicle rats. Laue et al. [251] reported that daily administration of RU486 for 2 weeks decreased food intake and body weight gain in obese, but not lean, 5-week old female Zucker rats. In contrast, Havel et al. [252] reported that daily administration of RU486 did not affect food intake, but did lower body weight in young, 5-week old male and female Zucker rats. There are a few studies investigating RU486 in Wistar rats, but only one study measured adipose depot mass. Stelmanska [253] abolished progesteroneinduced gains on body mass, inguinal fat mass, and expression of genes involved with lipogenesis in inguinal adipose tissue. Given that many of these studies were done in a rat model of obesity and all of the above studies were void of a physical activity component, my study presented in Chapter 3 provides new and novel data: 1) I present data on the effect of one week of RU486 treatment in male, Wistar rats in four adipose depots, 2) I present data on RU486 
as means of attenuating fat gains seen with a sudden decrease in physical activity, 3) I present an comprehensive transcriptome profile of RU486 treated rats for generation of future hypotheses.

As was mentioned in Chapter 3 , there was no difference in $11 \beta \mathrm{HSD}-1$ transcript expression between RUN and WL, but WL+GR BLOCK up-regulated $11 \beta$ HSD-1 mRNA compared to both RUN and WL. Perhaps, in and of itself, our wheel lock model does not provide a robust enough stimulus to affect $11 \beta \mathrm{HSD}-1$ expression. This finding may direct future research away from GCs and $11 \beta$ HSD-1 in this model.

\section{Conclusion}

In summary, I formulated my hypotheses and dissertation around four questions, which were addressed and answered in the two studies:

1) Does the way in which adipose tissue expands depend on the age of WL?

Yes. WL in 49-56 day old rats had no effect of wheel lock on omental, epididymal, and perirenal adipose tissue mass, mean adipocyte diameter, and total adipocyte number, while wheel lock on 70-77 day old rats resulted in epididymal and perirenal expansion via hyperplasia.

2) How does adipose tissue expand with 7 days of wheel lock? 
In 49-56 day old rats, epididymal adipose tissue likely expands by hypertrophy, which occurred in the 49-56 day old RUN and SED rats.

Hyperplasia was not detectable within EAT in 49-56 day old WL rats. In contrast, in 70-77 day old rats, EAT and PRAT expands by hyperplasia.

\section{3) Is adipose tissue expansion during 7 days of wheel lock healthy or} unhealthy? I suggest that this expansion is healthy in terms of a lack of inflammatory mRNA expression. An initial screen of three inflammatory mRNAs showed that there was no inflammatory response with wheel lock. A brief survey of inflammatory transcripts from the RNA-Seq data similarly showed no increase in inflammation during 7 days of wheel lock.

\section{4) Do glucocorticoids play a role in adipose tissue expansion that} occurs with 7 days of wheel lock? Blocking the glucocorticoid receptor attenuated total body fat gains as well as visceral depot mass gains, so we cannot rule out the role of glucocorticoids in this phenomenon. However, glucocorticoid block did not attenuate fat gains through the same pathway or mRNA transcript expression as voluntary wheel running did.

My overall hypothesis was that seven days of wheel lock in rats that are 49-56 days old and rats that are 70-77 days old would result in gains in visceral adipose mass seen in age-matched sedentary rats concurrent with an increase in adipocyte size and inflammatory mRNA expression. This hypothesis was not confirmed. Visceral adipose levels in wheel locked rats did not reach those of sedentary rats in either age group. Additionally, 49-56 day old rats showed 
different adipose expansion than 70-77 day old rats when compared to agematched sedentary and physically active rats. Finally, there was no inflammatory mRNA expression in either age group. I further hypothesized that glucocorticoid block would attenuate the visceral adipose depot gains seen in WL in 70-77 day old rats. This hypothesis was confirmed in that we saw attenuated visceral adipose depot gains with the glucocorticoid block, but the pathway by which the glucocorticoid block attenuated gains differed from voluntary running. 


\section{CHAPTER 5}

\section{Future Direction}

In a recent review from 2013, Efrat and Tepper [254] stated that,

"There is strong evidence that childhood overweight and obesity are risk factors for severe obesity over the whole life course. In fact, longitudinal studies have found that most overweight/obese children would become overweight and obese adults. There is a lack of coupling in the scientific literature between adipose tissue development and biology to obesity prevention and treatment strategies. This is of utmost importance, especially regarding childhood and adolescence, as the major scientific paradigm in studies of adiposity is that the major number of adipocytes is set for life at this early age."

Unfortunately, the work that has been done thus far is clearly not adequate, and we have not yet seen a decrease in the absolute number of obese and overweight children. It is my opinion that more research needs to be done to understand the interaction between physical activity and adipose tissue development in children and adolescents. In that regard, I will suggest a number of studies that can be performed using the WL model to address as-of-yet unknown answers to questions. My ideas are presented next. Each subsection represents a study that could be performed.

\section{RNA-Sequencing analysis between RUN and WL rats}

My committee agreed upon my dissertation telling a story. One part of my RNA-Seq data fit into the story and was told in Chapter 3. The study presented in Chapter 3 focused on 173 transcripts pertaining to cell cycle, proliferation, and 
differentiation, but there was much more data that I have access to from this RNA-Seq analysis. In fact, there are 16,610 transcripts that are shared by both RUN and WL rats (see Chapter 3). Using a filtering process described in Chapter 3, I found 118 differentially expressed transcripts (DETs), 74 of which were up-regulated in RUN rats compared to WL rats, and 44 of which were down-regulated in RUN rats compared to WL rats. In addition, there were 14 transcripts that met the filtering criteria that were exclusively expressed in RUN rats but not in WL rats and 21 transcripts that were exclusively expressed in WL rats but not in RUN rats. The top 10 transcripts for each of these categories are found in Chapter 3, Tables 6 and 7. A complete list of these transcripts is found in Appendix A.

Much of my analysis of RNA-Seq data that did not fit into my dissertation has already been completed. The list of all transcripts that met the filtering criteria outlined in Chapter 3 is listed in Appendix A. In addition, the top pathways identified by Ingenuity Pathway Analysis (IPA) are also listed in Appendix A. Unlike the primary purpose of the analysis in Chapter 3 (focus on cell cycle, proliferation, and differentiation transcripts), the primary purpose of this comprehensive analysis (looking at all transcritps) is to test the hypothesis that transcripts related to adipogenesis would be up-regulated in the WL rats. Additionally, RNA-Seq efforts were also performed in order to observe other nonadipogenic pathway transcriptomic differences within perirenal adipose tissue between RUN and WL rats in order to generate new hypotheses for future experiments using this model. 
In addition, 5781 unknown transcripts were present in the RNA-Seq analysis, meaning that a transcript was expressed in these rats, but when this transcript was compared to our transcript library, there was no match. Of these 5781 transcripts, 1353 had average RPKM values > 10 (the highest RPKM average was 1182.3994) and 2061 had average RP KM values between 1.0 and 9.99. This indicates that there are many, medium- and high-expressed transcripts that, as of yet, cannot be identified with current data bases. It is quite likely that one or two of these as-of-yet-unidentified transcripts could be integral in elucidating mechanisms by which $\mathrm{WL}$ accumulates visceral fat. It will eventually be possible to identify some, if not all, of these transcripts with indepth research by the Booth lab and many others.

\section{Investigate the adipocyte set-point hypothesis with WL}

As was discussed in Chapter 4, while it is accepted that puberty is a critical time of adipose tissue expansion [5, 233, 234], there is not universal consensus that the number of adipocytes is set for life at puberty $[6,43]$. While I have shown that WL induces hyperplasic adipose expansion in 70-77 day old rats, we do not know the effects of WL in young adult rats (older than 77 days of age). Does the adipocyte set-point hypothesis hold? Do rats maintain a steady number of adipocytes when WL is performed after the adipocyte number is 'set'? Human studies suggest that adipocyte expansion in adults occurs via 
hypertrophy [6] and only in extreme cases of obesity does hyperplasia occur [235].

My working hypothesis is that we have a relatively stable number of adipocytes that is set after puberty, and locking wheels in adulthood will lead to hypertrophic adipose expansion. This hypothesis can be tested by allowing rats access to a voluntary running wheel through 91 days of age. Rat literature suggests that adipocyte number plateaus around 12 weeks of age [10]. A subset of rats would undergo one week of WL at 91 days of age. A design similar to the one described in Chapter 2 would be employed to investigate body mass, lean mass, fat mass, \% body fat, OAT, EAT, and PRAT depot mass, adipocyte diameter, and depot adipocyte total adipocyte number.

Should we find that, in fact, WL does not induce visceral adipose expansion via hyperplasia in adult rats, it would support Spalding's findings [6] that there is a relatively tight regulation of adipocyte number after puberty. If we find that WL during adulthood induces hyperplasic visceral adipose expansion, a case can be made that adipose tissue is quite dynamic and has the ability to expand via hyperplasia into adulthood. Either way, the finding would fill a void in adipose literature and would help to shape communication to encourage physical activity in children in the context of life-long health. 


\section{Investigate whether daily physical activity through puberty protects from obesity later in life}

If a fetus is exposed to prenatal growth restraint, then provided with sufficient calories as a newborn, these babies develop spontaneous catch-up growth, but still have a marked reduction of SAT in early infancy [255]. This reduction in SAT during development has been suggested to confer long-term risk for metabolic diseases such as insulin resistance when calorie supplies become available $[256,257]$. When I learned this, I had the question, "Does maintaining a low set-point of whole-body total adipocyte number through and after puberty provide a protective effect or is it a risk factor for future metabolic disease?"

This is a follow-up to the previously suggested study \#2. If we find that adipocyte number is set for life after puberty, are rats and children "protected" from obesity or an unhealthy adipose tissue phenotype throughout life, or are they more at risk? As much as I would like to believe that daily physical activity through childhood and early adulthood is protective, the data, specifically that adulthood adipose tissue expansion via hypertrophy predominates [6], leads me to a different hypothesis. I hypothesize that a sedentary $(\mathrm{WL})$ condition in adulthood after previously undergoing daily physical activity, will lead to an hypertrophic visceral adipose tissue expansion accompanied with a "unhealthy," pro-inflammatory adipose tissue phenotype. 
The rationale for this hypothesis is that if: 1) a greater number of adipocytes at puberty increases risk for developing obesity later in life [7], 2) adipocyte number is set at puberty $[6,10]$ and to be determined the suggested study \#2), and 3) adipose tissue expansion can only occur through hypertrophy later in life (to be determined from the suggested study \#2) then, adult-onset adipose tissue expansion is likely to carry an unhealthy phenotype including increased expression of pro-inflammatory mRNAs.

\section{Investigate the effect of cyclic physical activity (activity-inactivity- activity-inactivity...) on adipose tissue.}

This study is also a follow up to proposed study \#2. If the effect of WL in young rats induces hyperplasia, what does the effect of repeated bouts of WL do? For example, if we WL a rat at 70-77 days of age (where I found both hyperplasic visceral adipose growth), then allow the rat access to a running wheel at 77-91days of age, then WL again at 91-97 days of age (results of proposed study \#2 will provide data on visceral adipose tissue response to $\mathrm{WL}$ at this age), will we see an exaggerated hyperplasic response (i.e. would WL done twice on rats exhibit more visceral total adipocyte number than WL done once?). This situation mimics life nicely; children are active during the summer, then are forced to sit quietly in school, so the public health message from this study has potential to be powerful.

Since no study has been done like this before, any hypothesis is purely speculation. However, there is a little information on exercise training in rats at 
70-77 days of age. Jenkins et al. recently published a study where they began exercise training OLETF rats at 19 weeks of age through 30-32 weeks of age [110]. They found that when compared to rats that were not exercise trained, rats that were exercise trained had lower omental, retroperitoneal, and epididymal fat depot masses but similar adipocyte diameters. This rat strain and age differs from the Wistars used in my proposed study \#4, but I am comfortable hypothesizing that running between the ages of 77-91 days would reduce visceral adipose mass. Sticking with my working hypothesis that rats have a relatively stable number of adipocytes that is set after puberty, increased activity (i.e. treadmill running like in Jenkins' study or WL in my studies) after puberty decreases visceral adipose mass, and locking wheels in adulthood will lead to hypertrophic adipose expansion, I hypothesize that RUN/WL/RUN/WL rats would exhibit greater visceral adipose depot masses and more visceral total adipocyte number than rats who had access to a voluntary running wheel the entire 97 days, but less visceral mass, but bigger adipocytes than rats who underwent only one bout of WL at either 70 days (Chapter 2) or 91 days (proposed experiment \#2).

\section{Other potentially interesting investigations}

5a. Investigate $W L$ effects on female rats. All of the experiments in my dissertation were performed on male, Wistar rats. These experiments could be repeated with female rats to determine if there is a similar effect or if there a sex- 
specific attenuation or augmentation of visceral adipose expansion induced by WL exists, as female Wistar rats voluntarily run almost twice the distances of male rats.

5b. Acute molecular changes $w / W L$. I employed one week of WL before looking at transcript profiles, but there may be interesting changes occurring within the first hours or 1-2 days of WL. Food intake data from Chapter 2 show a rapid decrease in food intake over 3 days. Feed efficiency also showed highly variable levels the first 3 days of WL. The same experimental design could be applied as described in Chapter 2, but rats were sacrificed earlier. Molecular markers could be investigated to help understand acute mechanisms of decreased activity in adipose tissue.

5c. Perform RNA-Seq on liver. Given the important role of the liver in fatty acid metabolism, turnover, and partitioning [258, 259], it would be interesting to investigate the transcriptome of livers of RUN and WL rats. Perhaps some insight could be gained into fatty acid metabolism and redistribution during WL in our model. Some work has already been done in regards to WL and liver. Laye et al. [95] found that one week of WL after 6 weeks of voluntary wheel running in Fischer 344 x Brown Norway F1 hybrid rats increased hepatic fatty acid oxidation above RUN and sedentary levels. Rector et al. [260] found that one week of WL after 16 weeks of voluntary wheel running in OLETF rats activated processes that initiate hepatic steatosis with no changes in body weight and no changes in omental and retroperitoneal adipose depots. Despite this insight, little is known about hepatic response to WL in our model of WL. 
$5 d$. Markers of hyperplasia in vivo. It would be interesting use in vivo measures of hyperplasia and hypertrophy. For example, we could use ${ }^{2} \mathrm{H}_{2} \mathrm{O}$ labeling and mass spectrometry analysis [261] and BrdU injections [262] in conjunction with WL. Using these methods could add to our understanding of adipose tissue expansion in the WL model and provide another line of evidence supporting my hyperplasia hypothesis. 


\section{REFERENCES}

1. Dumith, S.C., et al., Physical activity change during adolescence: a systematic review and a pooled analysis. Int J Epidemiol, 2011. 40(3): p. 685-98.

2. Chung, A.E., et al., Physical activity and BMI in a nationally representative sample of children and adolescents. Clin Pediatr (Phila), 2012. 51(2): $p$. 122-9.

3. Nader, P.R., et al., Moderate-to-vigorous physical activity from ages 9 to 15 years. JAMA, 2008. 300(3): p. 295-305.

4. Ogden, C.L., et al., Prevalence of obesity and trends in body mass index among US children and adolescents, 1999-2010. JAMA, 2012. 307(5): $p$. 483-90.

5. Knittle, J.L., et al., The growth of adipose tissue in children and adolescents. Cross-sectional and longitudinal studies of adipose cell number and size. J Clin Invest, 1979. 63(2): p. 239-46.

6. Spalding, K.L., et al., Dynamics of fat cell turnover in humans. Nature, 2008. 453(7196): p. 783-7.

7. Leunissen, R.W., et al., Fat mass accumulation during childhood determines insulin sensitivity in early adulthood. J Clin Endocrinol Metab, 2008. 93(2): p. 445-51.

8. Roberts, M.D., et al., Potential clinical translation of juvenile rodent inactivity models to study the onset of childhood obesity. Am J Physiol Regul Integr Comp Physiol, 2012. 303(3): p. R247-58.

9. Gregorio, B.M., et al., Maternal fish oil supplementation benefits programmed offspring from rat dams fed low-protein diet. Am J Obstet Gynecol, 2008. 199(1): p. 82 e1-7.

10. Greenwood, M.R. and J. Hirsch, Postnatal development of adipocyte cellularity in the normal rat. J Lipid Res, 1974. 15(5): p. 474-83. 
11. Harwood, H.J., Jr., The adipocyte as an endocrine organ in the regulation of metabolic homeostasis. Neuropharmacology, 2012. 63(1): p. 57-75.

12. Cannon, B. and J. Nedergaard, Brown adipose tissue: function and physiological significance. Physiol Rev, 2004. 84(1): p. 277-359.

13. Walden, T.B., et al., Recruited vs. nonrecruited molecular signatures of brown, "brite," and white adipose tissues. Am J Physiol Endocrinol Metab, 2012. 302(1): p. E19-31.

14. Wu, J., P. Cohen, and B.M. Spiegelman, Adaptive thermogenesis in adipocytes: is beige the new brown? Genes Dev, 2013. 27(3): p. 234-50.

15. Majka, S.M., Y. Barak, and D.J. Klemm, Concise review: adipocyte origins: weighing the possibilities. Stem Cells, 2011. 29(7): p. 1034-40.

16. Gimble, J.M., et al., Adipose-derived stromal/stem cells: A primer. Organogenesis, 2013. 9(1).

17. Calabro, P., et al., Adipose tissue-mediated inflammation: the missing link between obesity and cardiovascular disease? Intern Emerg Med, 2009. 4(1): p. 25-34.

18. Gustafson, B., et al., Inflammation and impaired adipogenesis in hypertrophic obesity in man. Am J Physiol Endocrinol Metab, 2009. 297(5): p. E999-E1003.

19. Strissel, K.J., et al., Adipocyte death, adipose tissue remodeling, and obesity complications. Diabetes, 2007. 56(12): p. 2910-8.

20. Engstrom, G., et al., Inflammation-sensitive plasma proteins, diabetes, and mortality and incidence of myocardial infarction and stroke: a population-based study. Diabetes, 2003. 52(2): p. 442-7.

21. Wozniak, S.E., et al., Adipose tissue: the new endocrine organ? A review article. Dig Dis Sci, 2009. 54(9): p. 1847-56. 
22. Liu, P.Y., et al., Evidence for the association between abdominal fat and cardiovascular risk factors in overweight and obese African American women. J Am Coll Nutr, 2012. 31(2): p. 126-32.

23. Batra, A. and B. Siegmund, The role of visceral fat. Dig Dis, 2012. 30(1): p. $70-4$.

24. Fox, C.S., et al., Abdominal visceral and subcutaneous adipose tissue compartments: association with metabolic risk factors in the Framingham Heart Study. Circulation, 2007. 116(1): p. 39-48.

25. Porter, S.A., et al., Abdominal subcutaneous adipose tissue: a protective fat depot? Diabetes Care, 2009. 32(6): p. 1068-75.

26. Yusuf, S., et al., Obesity and the risk of myocardial infarction in 27,000 participants from 52 countries: a case-control study. Lancet, 2005. 366(9497): p. 1640-9.

27. Rexrode, K.M., et al., Abdominal adiposity and coronary heart disease in women. JAMA, 1998. 280(21): p. 1843-8.

28. Canoy, D., et al., Body fat distribution and risk of coronary heart disease in men and women in the European Prospective Investigation Into Cancer and Nutrition in Norfolk cohort: a population-based prospective study. Circulation, 2007. 116(25): p. 2933-43.

29. Balkau, B., et al., International Day for the Evaluation of Abdominal Obesity (IDEA): a study of waist circumference, cardiovascular disease, and diabetes mellitus in 168,000 primary care patients in 63 countries. Circulation, 2007. 116(17): p. 1942-51.

30. Bergman, R.N., et al., Why visceral fat is bad: mechanisms of the metabolic syndrome. Obesity (Silver Spring), 2006. 14 Suppl 1: p. 16S$19 S$.

31. Haffner, S.M., Abdominal adiposity and cardiometabolic risk: do we have all the answers? Am J Med, 2007. 120(9 Suppl 1): p. S10-6; discussion S16-7. 
32. Mathieu, P., et al., Visceral obesity: the link among inflammation, hypertension, and cardiovascular disease. Hypertension, 2009. 53(4): p. 577-84.

33. Rutkowski, J.M., K.E. Davis, and P.E. Scherer, Mechanisms of obesity and related pathologies: the macro- and microcirculation of adipose tissue. FEBS J, 2009. 276(20): p. 5738-46.

34. Chaston, T.B. and J.B. Dixon, Factors associated with percent change in visceral versus subcutaneous abdominal fat during weight loss: findings from a systematic review. Int J Obes (Lond), 2008. 32(4): p. 619-28.

35. Gil, A., et al., Is adipose tissue metabolically different at different sites? Int J Pediatr Obes, 2011. 6 Suppl 1: p. 13-20.

36. Kabir, M., et al., Molecular evidence supporting the portal theory: a causative link between visceral adiposity and hepatic insulin resistance. Am J Physiol Endocrinol Metab, 2005. 288(2): p. E454-61.

37. Ukropec, J., et al., Adipose tissue and skeletal muscle plasticity modulates metabolic health. Arch Physiol Biochem, 2008. 114(5): p. 357-68.

38. Christiaens, V. and H.R. Lijnen, Angiogenesis and development of adipose tissue. Mol Cell Endocrinol, 2010. 318(1-2): p. 2-9.

39. Chen, L.L., et al., Lipid overaccumulation and drastic insulin resistance in adult catch-up growth rats induced by nutrition promotion after undernutrition. Metabolism, 2011. 60(4): p. 569-78.

40. Dulloo, A.G., Thrifty energy metabolism in catch-up growth trajectories to insulin and leptin resistance. Best Pract Res Clin Endocrinol Metab, 2008. 22(1): p. 155-71.

41. Otto, T.C. and M.D. Lane, Adipose development: from stem cell to adipocyte. Crit Rev Biochem Mol Biol, 2005. 40(4): p. 229-42.

42. Tang, Q.Q. and M.D. Lane, Adipogenesis: from stem cell to adipocyte. Annu Rev Biochem, 2012. 81: p. 715-36. 
43. Sun, K., C.M. Kusminski, and P.E. Scherer, Adipose tissue remodeling and obesity. J Clin Invest, 2011. 121(6): p. 2094-101.

44. Covas, D.T., et al., Multipotent mesenchymal stromal cells obtained from diverse human tissues share functional properties and gene-expression profile with CD146+ perivascular cells and fibroblasts. Exp Hematol, 2008. 36(5): p. 642-54.

45. Lin, C.S., et al., Defining adipose tissue-derived stem cells in tissue and in culture. Histol Histopathol, 2010. 25(6): p. 807-15.

46. Tang, Q.Q., T.C. Otto, and M.D. Lane, Commitment of C3H10T1/2 pluripotent stem cells to the adipocyte lineage. Proc Natl Acad Sci U S A, 2004. 101(26): p. 9607-11.

47. Davis, L.A. and N.I. Zur Nieden, Mesodermal fate decisions of a stem cell: the Wnt switch. Cell Mol Life Sci, 2008. 65(17): p. 2658-74.

48. Chung, Y.M., et al., Dietary D-psicose reduced visceral fat mass in highfat diet-induced obese rats. J Food Sci, 2012. 77(2): p. H53-8.

49. Shepherd, P.R., et al., Adipose cell hyperplasia and enhanced glucose disposal in transgenic mice overexpressing GLUT4 selectively in adipose tissue. J Biol Chem, 1993. 268(30): p. 22243-6.

50. Cartwright, A.L., Adipose cellularity in Gallus domesticus: investigations to control body composition in growing chickens. J Nutr, 1991. 121(9): p. 1486-97.

51. Roche, A.F., The adipocyte-number hypothesis. Child Dev, 1981. 52(1): p. 31-43.

52. Skurk, T., et al., Relationship between adipocyte size and adipokine expression and secretion. J Clin Endocrinol Metab, 2007. 92(3): p. 102333.

53. Slawik, M. and A.J. Vidal-Puig, Adipose tissue expandability and the metabolic syndrome. Genes Nutr, 2007. 2(1): p. 41-5. 
54. Kim, J.Y., et al., Obesity-associated improvements in metabolic profile through expansion of adipose tissue. J Clin Invest, 2007. 117(9): p. 262137.

55. Virtue, S. and A. Vidal-Puig, Adipose tissue expandability, lipotoxicity and the Metabolic Syndrome--an allostatic perspective. Biochim Biophys Acta, 2010. 1801(3): p. 338-49.

56. Summermatter, S., et al., Adipose tissue plasticity during catch-up fat driven by thrifty metabolism: relevance for muscle-adipose glucose redistribution during catch-up growth. Diabetes, 2009. 58(10): p. 2228-37.

57. Gluckman, P.D., et al., Effect of in utero and early-life conditions on adult health and disease. N Engl J Med, 2008. 359(1): p. 61-73.

58. Gregoire, F.M., C.M. Smas, and H.S. Sul, Understanding adipocyte differentiation. Physiol Rev, 1998. 78(3): p. 783-809.

59. Dulloo, A.G., Adipose tissue plasticity in catch-up-growth trajectories to metabolic syndrome: hyperplastic versus hypertrophic catch-up fat. Diabetes, 2009. 58(5): p. 1037-9.

60. Laye, M.J., et al., Inactivity induces increases in abdominal fat. J Appl Physiol, 2007. 102(4): p. 1341-7.

61. Kump, D.S. and F.W. Booth, Sustained rise in triacylglycerol synthesis and increased epididymal fat mass when rats cease voluntary wheel running. J Physiol, 2005. 565(Pt 3): p. 911-25.

62. Olsen, R.H., et al., Metabolic responses to reduced daily steps in healthy nonexercising men. JAMA, 2008. 299(11): p. 1261-3.

63. Kyle, U.G. and C. Pichard, The Dutch Famine of 1944-1945: a pathophysiological model of long-term consequences of wasting disease. Curr Opin Clin Nutr Metab Care, 2006. 9(4): p. 388-94. 
64. Dulloo, A.G., et al., The thrifty 'catch-up fat' phenotype: its impact on insulin sensitivity during growth trajectories to obesity and metabolic syndrome. Int J Obes (Lond), 2006. 30 Suppl 4: p. S23-35.

65. Isganaitis, E., et al., Accelerated postnatal growth increases lipogenic gene expression and adipocyte size in low-birth weight mice. Diabetes, 2009. 58(5): p. 1192-200.

66. Jakicic, J.M., The effect of physical activity on body weight. Obesity (Silver Spring), 2009. 17 Suppl 3: p. S34-8.

67. Lee, D.C., X. Sui, and S.N. Blair, Does physical activity ameliorate the health hazards of obesity? Br J Sports Med, 2009. 43(1): p. 49-51.

68. Antic, S., et al., [Exercise as a therapeutic option in cardiometabolic risk reduction]. Med Pregl, 2009. 62 Suppl 3: p. 59-65.

69. McCall, A. and R. Raj, Exercise for prevention of obesity and diabetes in children and adolescents. Clin Sports Med, 2009. 28(3): p. 393-421.

70. Hamer, M. and G. O'Donovan, Cardiorespiratory fitness and metabolic risk factors in obesity. Curr Opin Lipidol, 2010. 21(1): p. 1-7.

71. Kay, S.J. and M.A. Fiatarone Singh, The influence of physical activity on abdominal fat: a systematic review of the literature. Obes Rev, 2006. 7(2): p. $183-200$.

72. Duclos, M., C. Gouarne, and D. Bonnemaison, Acute and chronic effects of exercise on tissue sensitivity to glucocorticoids. J Appl Physiol, 2003. 94(3): p. 869-75.

73. Campbell, J.E., et al., Endurance exercise training increases adipose tissue glucocorticoid exposure: adaptations that facilitate lipolysis. Metabolism, 2009. 58(5): p. 651-60.

74. Bjorntorp, P. and R. Rosmond, Obesity and cortisol. Nutrition, 2000. 16(10): p. 924-36. 
75. Campbell, J.E., et al., Regular exercise prevents the development of hyperglucocorticoidemia via adaptations in the brain and adrenal glands in male Zucker diabetic fatty rats. Am J Physiol Regul Integr Comp Physiol, 2010. 299(1): p. R168-76.

76. Xu, C., et al., Direct effect of glucocorticoids on lipolysis in adipocytes. Mol Endocrinol, 2009. 23(8): p. 1161-70.

77. Wiper-Bergeron, N., et al., Glucocorticoid-stimulated preadipocyte differentiation is mediated through acetylation of C/EBPbeta by GCN5. Proc Natl Acad Sci U S A, 2007. 104(8): p. 2703-8.

78. Pedersen, S.B., M. Jonler, and B. Richelsen, Characterization of regional and gender differences in glucocorticoid receptors and lipoprotein lipase activity in human adipose tissue. J Clin Endocrinol Metab, 1994. 78(6): p. 1354-9.

79. van Jaarsveld, C.H., et al., Perceived stress and weight gain in adolescence: a longitudinal analysis. Obesity (Silver Spring), 2009. 17(12): p. 2155-61.

80. Stewart, P.M. and S. Petersenn, Rationale for treatment and therapeutic options in Cushing's disease. Best Pract Res Clin Endocrinol Metab, 2009. 23 Suppl 1: p. S15-22.

81. McDonough, A.K., J.R. Curtis, and K.G. Saag, The epidemiology of glucocorticoid-associated adverse events. Curr Opin Rheumatol, 2008. 20(2): p. 131-7.

82. Friedman, T.C., et al., Carbohydrate and lipid metabolism in endogenous hypercortisolism: shared features with metabolic syndrome $X$ and NIDDM. Endocr J, 1996. 43(6): p. 645-55.

83. Abad, V., et al., Glucocorticoid excess during adolescence leads to a major persistent deficit in bone mass and an increase in central body fat. $\mathrm{J}$ Bone Miner Res, 2001. 16(10): p. 1879-85. 
84. Peckett, A.J., D.C. Wright, and M.C. Riddell, The effects of glucocorticoids on adipose tissue lipid metabolism. Metabolism, 2011. 60(11): p. 1500-10.

85. Covar, R.A., et al., Risk factors associated with glucocorticoid-induced adverse effects in children with severe asthma. J Allergy Clin Immunol, 2000. 106(4): p. 651-9.

86. Hopkins, R.L. and M.C. Leinung, Exogenous Cushing's syndrome and glucocorticoid withdrawal. Endocrinol Metab Clin North Am, 2005. 34(2): p. 371-84, ix.

87. Shiff, N.J., et al., Glucocorticoid-related changes in body mass index among children and adolescents with rheumatic diseases. Arthritis Care Res (Hoboken), 2013. 65(1): p. 113-21.

88. Langley, S.C. and D.A. York, Effects of antiglucocorticoid RU 486 on development of obesity in obese fa/fa Zucker rats. Am J Physiol, 1990. 259(3 Pt 2): p. R539-44.

89. Zanato, V.F., et al., Sexual development of male Wistar rats. Braz J Med Biol Res, 1994. 27(5): p. 1273-80.

90. Hotamisligil, G.S., Inflammation and metabolic disorders. Nature, 2006. 444(7121): p. 860-7.

91. Ouchi, N., et al., Adipokines in inflammation and metabolic disease. Nat Rev Immunol, 2011. 11(2): p. 85-97.

92. Klein, S., et al., Waist Circumference and Cardiometabolic Risk: a Consensus Statement from Shaping America's Health: Association for Weight Management and Obesity Prevention; NAASO, the Obesity Society; the American Society for Nutrition; and the American Diabetes Association. Obesity (Silver Spring), 2007. 15(5): p. 1061-7.

93. Thomas, E.L., et al., Excess body fat in obese and normal-weight subjects. Nutr Res Rev, 2012. 25(1): p. 150-61. 
94. Symonds, M.E., et al., Adipose tissue development--impact of the early life environment. Prog Biophys Mol Biol, 2011. 106(1): p. 300-6.

95. Laye, M.J., et al., Cessation of daily wheel running differentially alters fat oxidation capacity in liver, muscle, and adipose tissue. J Appl Physiol, 2009. 106(1): p. 161-8.

96. Toedebusch, R.G., et al., Postprandial leucine and insulin responses and toxicological effects of a novel whey protein hydrolysate-based supplement in rats. J Int Soc Sports Nutr, 2012. 9(1): p. 24.

97. Mize, R.R., et al., The role of nitric oxide in development of the patchcluster system and retinocollicular pathways in the rodent superior colliculus. Prog Brain Res, 1998. 118: p. 133-52.

98. MacLean, P.S., et al., Regular exercise attenuates the metabolic drive to regain weight after long-term weight loss. Am J Physiol Regul Integr Comp Physiol, 2009. 297(3): p. R793-802.

99. Barbatelli, G., et al., The emergence of cold-induced brown adipocytes in mouse white fat depots is determined predominantly by white to brown adipocyte transdifferentiation. Am J Physiol Endocrinol Metab, 2010. 298(6): p. E1244-53.

100. Nader, P.R., R.H. Bradley, and R.M. Houts, Data error in study of moderate-to-vigorous physical activity from ages 9 to 15 years. JAMA, 2009. 301(20): p. 2094-5.

101. Jackman, M.R., et al., Weight regain after sustained weight reduction is accompanied by suppressed oxidation of dietary fat and adipocyte hyperplasia. Am J Physiol Regul Integr Comp Physiol, 2008. 294(4): p. R1117-29.

102. Okuno, A., et al., Troglitazone increases the number of small adipocytes without the change of white adipose tissue mass in obese Zucker rats. $J$ Clin Invest, 1998. 101(6): p. 1354-61. 
103. Tchkonia, T., et al., Fat depot-specific characteristics are retained in strains derived from single human preadipocytes. Diabetes, 2006. 55(9): p. 2571-8.

104. Ye, J., et al., Hypoxia is a potential risk factor for chronic inflammation and adiponectin reduction in adipose tissue of ob/ob and dietary obese mice. Am J Physiol Endocrinol Metab, 2007. 293(4): p. E1118-28.

105. Hosogai, N., et al., Adipose tissue hypoxia in obesity and its impact on adipocytokine dysregulation. Diabetes, 2007. 56(4): p. 901-11.

106. Halberg, N., et al., Hypoxia-inducible factor 1alpha induces fibrosis and insulin resistance in white adipose tissue. Mol Cell Biol, 2009. 29(16): p. 4467-83.

107. Speretta, G.F., et al., The effects of exercise modalities on adiposity in obese rats. Clinics (Sao Paulo), 2012. 67(12): p. 1469-77.

108. Sampey, B.P., et al., Cafeteria diet is a robust model of human metabolic syndrome with liver and adipose inflammation: comparison to high-fat diet. Obesity (Silver Spring), 2011. 19(6): p. 1109-17.

109. Ben-Shlomo, S., et al., Role of glucose-dependent insulinotropic polypeptide in adipose tissue inflammation of dipeptidylpeptidase 4deficient rats. Obesity (Silver Spring), 2013.

110. Jenkins, N.T., et al., Effects of endurance exercise training, metformin, and their combination on adipose tissue leptin and IL-10 secretion in OLETF rats. J Appl Physiol, 2012. 113(12): p. 1873-83.

111. Girard, F., et al., Cyclin $A$ is required for the onset of DNA replication in mammalian fibroblasts. Cell, 1991. 67(6): p. 1169-79.

112. Pagano, M., et al., Cyclin $A$ is required at two points in the human cell cycle. EMBO J, 1992. 11(3): p. 961-71. 
113. Zhu, M., et al., Adipogenic signaling in rat white adipose tissue: modulation by aging and calorie restriction. Exp Gerontol, 2007. 42(8): $p$. 733-44.

114. Lofgren, P., et al., Long-term prospective and controlled studies demonstrate adipose tissue hypercellularity and relative leptin deficiency in the postobese state. J Clin Endocrinol Metab, 2005. 90(11): p. 6207-13.

115. DiGirolamo, M., et al., Qualitative regional differences in adipose tissue growth and cellularity in male Wistar rats fed ad libitum. Am J Physiol, 1998. 274(5 Pt 2): p. R1460-7.

116. Morimoto, C., T. Tsujita, and H. Okuda, Antilipolytic actions of insulin on basal and hormone-induced lipolysis in rat adipocytes. J Lipid Res, 1998. 39(5): p. 957-62.

117. Lofgren, P., et al., Prospective and controlled studies of the actions of insulin and catecholamine in fat cells of obese women following weight reduction. Diabetologia, 2005. 48(11): p. 2334-42.

118. Olefsky, J.M., Insensitivity of large rat adipocytes to the antilipolytic effects of insulin. J Lipid Res, 1977. 18(4): p. 459-64.

119. MacLean, P.S., et al., Peripheral metabolic responses to prolonged weight reduction that promote rapid, efficient regain in obesity-prone rats. Am $\mathrm{J}$ Physiol Regul Integr Comp Physiol, 2006. 290(6): p. R1577-88.

120. Patterson, C.M., A.A. Dunn-Meynell, and B.E. Levin, Three weeks of early-onset exercise prolongs obesity resistance in DIO rats after exercise cessation. Am J Physiol Regul Integr Comp Physiol, 2008. 294(2): p. R290-301.

121. MacLean, P.S., A peripheral perspective of weight regain. Am J Physiol Regul Integr Comp Physiol, 2005. 288(6): p. R1447-9.

122. Blundell, J.E., et al., Role of resting metabolic rate and energy expenditure in hunger and appetite control: a new formulation. Dis Model Mech, 2012. 5(5): p. 608-13. 
123. Alberga, A.S., et al., Overweight and obese teenagers: why is adolescence a critical period? Pediatr Obes, 2012. 7(4): p. 261-73.

124. Andrews, R.C. and B.R. Walker, Glucocorticoids and insulin resistance: old hormones, new targets. Clin Sci (Lond), 1999. 96(5): p. 513-23.

125. Bollen, M., S. Keppens, and W. Stalmans, Specific features of glycogen metabolism in the liver. Biochem J, 1998. 336 ( Pt 1): p. 19-31.

126. Tomlinson, J.W. and P.M. Stewart, Modulation of glucocorticoid action and the treatment of type-2 diabetes. Best Pract Res Clin Endocrinol Metab, 2007. 21(4): p. 607-19.

127. Kotelevtsev, Y., et al., 11beta-hydroxysteroid dehydrogenase type 1 knockout mice show attenuated glucocorticoid-inducible responses and resist hyperglycemia on obesity or stress. Proc Natl Acad Sci U S A, 1997. 94(26): p. 14924-9.

128. Morton, N.M., et al., Novel adipose tissue-mediated resistance to dietinduced visceral obesity in 11 beta-hydroxysteroid dehydrogenase type 1 deficient mice. Diabetes, 2004. 53(4): p. 931-8.

129. Morton, N.M., et al., Improved lipid and lipoprotein profile, hepatic insulin sensitivity, and glucose tolerance in 11beta-hydroxysteroid dehydrogenase type 1 null mice. J Biol Chem, 2001. 276(44): p. 41293300.

130. Slavin, B.G., J.M. Ong, and P.A. Kern, Hormonal regulation of hormonesensitive lipase activity and mRNA levels in isolated rat adipocytes. J Lipid Res, 1994. 35(9): p. 1535-41.

131. Gaillard, D., et al., Control of terminal differentiation of adipose precursor cells by glucocorticoids. J Lipid Res, 1991. 32(4): p. 569-79.

132. Spitz, I.M., Proven and potential clinical applications of mifpristone (RU486). Rev Endocr Metab Disord, 2002. 3(3): p. 267-75. 
133. Clapham, J.C. and N.C. Turner, Effects of the glucocorticoid II receptor antagonist mifepristone on hypertension in the obese Zucker rat. $\mathrm{J}$ Pharmacol Exp Ther, 1997. 282(3): p. 1503-8.

134. Gettys, T.W., et al., RU-486 (Mifepristone) ameliorates diabetes but does not correct deficient beta-adrenergic signalling in adipocytes from mature C57BL/6J-ob/ob mice. Int J Obes Relat Metab Disord, 1997. 21(10): p. 865-73.

135. Okada, S., D.A. York, and G.A. Bray, Mifepristone (RU 486), a blocker of type II glucocorticoid and progestin receptors, reverses a dietary form of obesity. Am J Physiol, 1992. 262(6 Pt 2): p. R1106-10.

136. Song, H.K., et al., Deep RNA sequencing reveals novel cardiac transcriptomic signatures for physiological and pathological hypertrophy. PLoS One, 2012. 7(4): p. e35552.

137. Dallman, M.F., et al., Minireview: glucocorticoids--food intake, abdominal obesity, and wealthy nations in 2004. Endocrinology, 2004. 145(6): $p$. 2633-8.

138. Krawiec, B.J., et al., Hindlimb casting decreases muscle mass in part by proteasome-dependent proteolysis but independent of protein synthesis. Am J Physiol Endocrinol Metab, 2005. 289(6): p. E969-80.

139. Atkinson, H.C. and B.J. Waddell, Circadian variation in basal plasma corticosterone and adrenocorticotropin in the rat: sexual dimorphism and changes across the estrous cycle. Endocrinology, 1997. 138(9): p. 3842-8.

140. Rustemeyer, S.M., et al., Effects of dietary aflatoxin on the hepatic expression of apoptosis genes in growing barrows. J Anim Sci, 2011. 89(4): p. 916-25.

141. Mortazavi, A., et al., Mapping and quantifying mammalian transcriptomes by RNA-Seq. Nat Methods, 2008. 5(7): p. 621-8.

142. Hackett, N.R., et al., RNA-Seq quantification of the human small airway epithelium transcriptome. BMC Genomics, 2012. 13: p. 82. 
143. Jabbari, A., et al., Transcriptional profiling of psoriasis using RNA-seq reveals previously unidentified differentially expressed genes. J Invest Dermatol, 2012. 132(1): p. 246-9.

144. Hampton, M., et al., Deep sequencing the transcriptome reveals seasonal adaptive mechanisms in a hibernating mammal. PLoS One, 2011. 6(10): p. e27021.

145. Asagami, T., et al., Selective Glucocorticoid Receptor (GR-II) Antagonist Reduces Body Weight Gain in Mice. J Nutr Metab, 2011. 2011: p. 235389.

146. Gross, C., et al., Mifepristone reduces weight gain and improves metabolic abnormalities associated with risperidone treatment in normal men. Obesity (Silver Spring), 2010. 18(12): p. 2295-300.

147. Kuo, T., C.A. Harris, and J.C. Wang, Metabolic functions of glucocorticoid receptor in skeletal muscle. Mol Cell Endocrinol, 2013.

148. Konagaya, M. and S.R. Max, A possible role for endogenous glucocorticoids in orchiectomy-induced atrophy of the rat levator ani muscle: studies with $R U$ 38486, a potent and selective antiglucocorticoid. J Steroid Biochem, 1986. 25(3): p. 305-8.

149. Macfarlane, D.P., S. Forbes, and B.R. Walker, Glucocorticoids and fatty acid metabolism in humans: fuelling fat redistribution in the metabolic syndrome. J Endocrinol, 2008. 197(2): p. 189-204.

150. Shvetsov, E.V., [Anatomy and topography of external iliac lymph nodes in adults]. Arkh Anat Gistol Embriol, 1991. 100(7-8): p. 50-7.

151. Fraser, R., et al., Cortisol effects on body mass, blood pressure, and cholesterol in the general population. Hypertension, 1999. 33(6): p. 13648.

152. Staab, C.A. and E. Maser, 11beta-Hydroxysteroid dehydrogenase type 1 is an important regulator at the interface of obesity and inflammation. $\mathrm{J}$ Steroid Biochem Mol Biol, 2010. 119(1-2): p. 56-72. 
153. Walker, B.R. and R. Andrew, Tissue production of cortisol by 11betahydroxysteroid dehydrogenase type 1 and metabolic disease. Ann N Y Acad Sci, 2006. 1083: p. 165-84.

154. Wake, D.J. and B.R. Walker, 11 beta-hydroxysteroid dehydrogenase type 1 in obesity and the metabolic syndrome. Mol Cell Endocrinol, 2004. 215(1-2): p. 45-54.

155. Robitaille, J., et al., Molecular screening of the 11beta-HSD1 gene in men characterized by the metabolic syndrome. Obes Res, 2004. 12(10): p. 1570-5.

156. Berthiaume, M., et al., Depot-specific modulation of rat intraabdominal adipose tissue lipid metabolism by pharmacological inhibition of 11 betahydroxysteroid dehydrogenase type 1. Endocrinology, 2007. 148(5): $p$. 2391-7.

157. Bagnato, C. and R.A. Igal, Overexpression of diacylglycerol acyltransferase-1 reduces phospholipid synthesis, proliferation, and invasiveness in simian virus 40-transformed human lung fibroblasts. J Biol Chem, 2003. 278(52): p. 52203-11.

158. Kusunoki, J., A. Kanatani, and D.E. Moller, Modulation of fatty acid metabolism as a potential approach to the treatment of obesity and the metabolic syndrome. Endocrine, 2006. 29(1): p. 91-100.

159. Chen, H.C. and R.V. Farese, Jr., Inhibition of triglyceride synthesis as a treatment strategy for obesity: lessons from DGAT1-deficient mice. Arterioscler Thromb Vasc Biol, 2005. 25(3): p. 482-6.

160. Alsted, T.J., et al., Adipose triglyceride lipase in human skeletal muscle is upregulated by exercise training. Am J Physiol Endocrinol Metab, 2009. 296(3): p. E445-53.

161. Yu, Y.H. and H.N. Ginsberg, The role of acyl-CoA:diacylglycerol acyltransferase (DGAT) in energy metabolism. Ann Med, 2004. 36(4): p. $252-61$. 
162. Yu, Y.H., et al., Posttranscriptional control of the expression and function of diacylglycerol acyltransferase-1 in mouse adipocytes. J Biol Chem, 2002. 277(52): p. 50876-84.

163. Newhall, K.J., et al., Deletion of the Rllbeta-subunit of protein kinase $A$ decreases body weight and increases energy expenditure in the obese, leptin-deficient ob/ob mouse. Mol Endocrinol, 2005. 19(4): p. 982-91.

164. Planas, J.V., et al., Mutation of the Rllbeta subunit of protein kinase $A$ differentially affects lipolysis but not gene induction in white adipose tissue. J Biol Chem, 1999. 274(51): p. 36281-7.

165. Chu, S., et al., FSH-regulated gene expression profiles in ovarian tumours and normal ovaries. Mol Hum Reprod, 2002. 8(5): p. 426-33.

166. Martinez, C.S., et al., Growth hormone STAT5-mediated signaling and its modulation in mice liver during the growth period. Growth Horm IGF Res, 2013. 23(1-2): p. 19-28.

167. Mui, A.L., The role of STATs in proliferation, differentiation, and apoptosis. Cell Mol Life Sci, 1999. 55(12): p. 1547-58.

168. Stephens, J.M., R.F. Morrison, and P.F. Pilch, The expression and regulation of STATS during 3T3-L1 adipocyte differentiation. J Biol Chem, 1996. 271(18): p. 10441-4.

169. Nanbu-Wakao, R., et al., Stimulation of 3T3-L1 adipogenesis by signal transducer and activator of transcription 5. Mol Endocrinol, 2002. 16(7): $p$. 1565-76.

170. Teglund, S., et al., Stat5a and Stat5b proteins have essential and nonessential, or redundant, roles in cytokine responses. Cell, 1998. 93(5): p. 841-50.

171. Campbell, P.K., et al., Mutation of a novel gene results in abnormal development of spermatid flagella, loss of intermale aggression and reduced body fat in mice. Genetics, 2002. 162(1): p. 307-20. 
172. Hwang, G.W., et al., Ubiquitin-conjugating enzyme Cdc34 mediates methylmercury resistance in Saccharomyces cerevisiae by increasing Whi2 degradation. J Toxicol Sci, 2012. 37(6): p. 1283-6.

173. Skaar, J.R. and M. Pagano, Control of cell growth by the SCF and APC/C ubiquitin ligases. Curr Opin Cell Biol, 2009. 21(6): p. 816-24.

174. Bieganowski, P., et al., Cdc123 and checkpoint forkhead associated with RING proteins control the cell cycle by controlling elF2gamma abundance. J Biol Chem, 2004. 279(43): p. 44656-66.

175. Tanguay, P.L., G. Rodier, and S. Meloche, C-terminal domain phosphorylation of ERK3 controlled by Cdk1 and Cdc14 regulates its stability in mitosis. Biochem J, 2010. 428(1): p. 103-11.

176. Santamaria, D., et al., Cdk1 is sufficient to drive the mammalian cell cycle. Nature, 2007. 448(7155): p. 811-5.

177. Joseph, V.T., A combined tubularized/onlay graft technique for total correction of severe hypospadias. J Pediatr Surg, 1999. 34(6): p. 992-5.

178. Tumurbaatar, I., et al., Human Cdc14B promotes progression through mitosis by dephosphorylating Cdc25 and regulating Cdk1/cyclin B activity. PLoS One, 2011. 6(2): p. e14711.

179. Chua, S.S., et al., Cdc25B as a steroid receptor coactivator. Vitam Horm, 2004. 68: p. 231-56.

180. Hansen, C.A., J. Bartek, and S. Jensen, $A$ functional link between the human cell cycle-regulatory phosphatase Cdc14A and the atypical mitogen-activated kinase Erk3. Cell Cycle, 2008. 7(3): p. 325-34.

181. Mayer, T.U., et al., Small molecule inhibitor of mitotic spindle bipolarity identified in a phenotype-based screen. Science, 1999. 286(5441): p. 9714.

182. Rath, O. and F. Kozielski, Kinesins and cancer. Nat Rev Cancer, 2012. 12(8): p. 527-39. 
183. Yim, H. and R.L. Erikson, Regulation of the final stage of mitosis by components of the pre-replicative complex and a polo kinase. Cell Cycle, 2011. 10(9): p. 1374-7.

184. Barr, F.A., H.H. Sillje, and E.A. Nigg, Polo-like kinases and the orchestration of cell division. Nat Rev Mol Cell Biol, 2004. 5(6): p. 429-40.

185. Mardin, B.R. and E. Schiebel, Breaking the ties that bind: new advances in centrosome biology. J Cell Biol, 2012. 197(1): p. 11-8.

186. Yim, H. and R.L. Erikson, Cell division cycle 6, a mitotic substrate of pololike kinase 1, regulates chromosomal segregation mediated by cyclindependent kinase 1 and separase. Proc Natl Acad Sci U S A, 2010. 107(46): p. 19742-7.

187. Sherr, C.J. and J.M. Roberts, Living with or without cyclins and cyclindependent kinases. Genes Dev, 2004. 18(22): p. 2699-711.

188. Morrison, R.F. and S.R. Farmer, Hormonal signaling and transcriptional control of adipocyte differentiation. J Nutr, 2000. 130(12): p. 3116S$3121 \mathrm{~S}$.

189. White, U.A. and J.M. Stephens, Transcriptional factors that promote formation of white adipose tissue. Mol Cell Endocrinol, 2010. 318(1-2): $p$. 10-4.

190. Mejhert, N., et al., Mapping of the fibroblast growth factors in human white adipose tissue. J Clin Endocrinol Metab, 2010. 95(5): p. 2451-7.

191. Liu, Y., et al., Lentiviral-mediated gene transfer into human adiposederived stem cells: role of NELL1 versus BMP2 in osteogenesis and adipogenesis in vitro. Acta Biochim Biophys Sin (Shanghai), 2012. 44(10): p. 856-65.

192. Tseng, Y.H., et al., New role of bone morphogenetic protein 7 in brown adipogenesis and energy expenditure. Nature, 2008. 454(7207): p. 10004. 
193. Ravnik, S.E. and D.J. Wolgemuth, Regulation of meiosis during mammalian spermatogenesis: the A-type cyclins and their associated cyclin-dependent kinases are differentially expressed in the germ-cell lineage. Dev Biol, 1999. 207(2): p. 408-18.

194. Davalos, V., et al., Human SMC2 protein, a core subunit of human condensin complex, is a novel transcriptional target of the WNT signaling pathway and a new therapeutic target. J Biol Chem, 2012. 287(52): p. 43472-81.

195. Wang, J., et al., Inhibition of activated pericentromeric SINE/Alu repeat transcription in senescent human adult stem cells reinstates self-renewal. Cell Cycle, 2011. 10(17): p. 3016-30.

196. Zhu, P., et al., Angiopoietin-like 4: a decade of research. Biosci Rep, 2012. 32(3): p. 211-9.

197. Gray, N.E., et al., Angiopoietin-like 4 (Angpt/4) protein is a physiological mediator of intracellular lipolysis in murine adipocytes. J Biol Chem, 2012. 287(11): p. 8444-56.

198. Kersten, S., et al., Characterization of the fasting-induced adipose factor FIAF, a novel peroxisome proliferator-activated receptor target gene. J Biol Chem, 2000. 275(37): p. 28488-93.

199. Yoon, J.C., et al., Peroxisome proliferator-activated receptor gamma target gene encoding a novel angiopoietin-related protein associated with adipose differentiation. Mol Cell Biol, 2000. 20(14): p. 5343-9.

200. Willer, C.J., et al., Newly identified loci that influence lipid concentrations and risk of coronary artery disease. Nat Genet, 2008. 40(2): p. 161-9.

201. Kathiresan, S., et al., Common variants at 30 loci contribute to polygenic dyslipidemia. Nat Genet, 2009. 41(1): p. 56-65.

202. Romeo, S., et al., Population-based resequencing of ANGPTL4 uncovers variations that reduce triglycerides and increase HDL. Nat Genet, 2007. 39(4): p. 513-6. 
203. Yoshida, K., et al., Angiopoietin-like protein 4 is a potent hyperlipidemiainducing factor in mice and inhibitor of lipoprotein lipase. J Lipid Res, 2002. 43(11): p. 1770-2.

204. Teslovich, T.M., et al., Biological, clinical and population relevance of 95 loci for blood lipids. Nature, 2010. 466(7307): p. 707-13.

205. Mandard, S., et al., The direct peroxisome proliferator-activated receptor target fasting-induced adipose factor (FIAF/PGAR/ANGPTL4) is present in blood plasma as a truncated protein that is increased by fenofibrate treatment. J Biol Chem, 2004. 279(33): p. 34411-20.

206. Mandard, S., et al., The fasting-induced adipose factor/angiopoietin-like protein 4 is physically associated with lipoproteins and governs plasma lipid levels and adiposity. J Biol Chem, 2006. 281(2): p. 934-44.

207. Dutton, S. and P. Trayhurn, Regulation of angiopoietin-like protein 4/fasting-induced adipose factor (Angpt/4/FIAF) expression in mouse white adipose tissue and 3T3-L1 adipocytes. Br J Nutr, 2008. 100(1): $\mathrm{p}$. 18-26.

208. Pal, M., et al., Angiopoietin-like 4 regulates epidermal differentiation. PLoS One, 2011. 6(9): p. e25377.

209. Koliwad, S.K., et al., Angiopoietin-like 4 (ANGPTL4, fasting-induced adipose factor) is a direct glucocorticoid receptor target and participates in glucocorticoid-regulated triglyceride metabolism. J Biol Chem, 2009. 284(38): p. 25593-601.

210. Torres, R.C., et al., Activation of PPARgamma by restores mast cell numbers and reactivity in alloxan-diabetic rats by reducing the systemic glucocorticoid levels. Eur J Pharmacol, 2012. 691(1-3): p. 261-7.

211. Rather, M.I., et al., Oncogenic microRNA-155 down-regulates tumor suppressor CDC73 and promotes oral squamous cell carcinoma cell proliferation: implications for cancer therapeutics. J Biol Chem, 2013. 288(1): p. 608-18. 
212. Wang, P., et al., Parafibromin, a component of the human PAF complex, regulates growth factors and is required for embryonic development and survival in adult mice. Mol Cell Biol, 2008. 28(9): p. 2930-40.

213. Addeo, R., et al., Glucocorticoids induce G1 arrest of lymphoblastic cells through retinoblastoma protein $R b 1$ dephosphorylation in childhood acute lymphoblastic leukemia in vivo. Cancer Biol Ther, 2004. 3(5): p. 470-6.

214. Cobrinik, D., Pocket proteins and cell cycle control. Oncogene, 2005. 24(17): p. 2796-809.

215. Hallenborg, P., et al., The tumor suppressors $p R B$ and $p 53$ as regulators of adipocyte differentiation and function. Expert Opin Ther Targets, 2009. 13(2): p. 235-46.

216. Dali-Youcef, N., et al., Adipose tissue-specific inactivation of the retinoblastoma protein protects against diabesity because of increased energy expenditure. Proc Natl Acad Sci U S A, 2007. 104(25): p. 10703-8.

217. Calo, E., et al., $R b$ regulates fate choice and lineage commitment in vivo. Nature, 2010. 466(7310): p. 1110-4.

218. Moreno-Navarrete, J.M., et al., Decreased RB1 mRNA, Protein, and Activity Reflect Obesity-Induced Altered Adipogenic Capacity in Human Adipose Tissue. Diabetes, 2013.

219. Nutrition and you: trends 2000. ADA's Public Relations Team. J Am Diet Assoc, 2000. 100(6): p. 626-7.

220. Makris, A. and G.D. Foster, Dietary approaches to the treatment of obesity. Psychiatr Clin North Am, 2011. 34(4): p. 813-27.

221. Brambilla, P., G. Pozzobon, and A. Pietrobelli, Physical activity as the main therapeutic tool for metabolic syndrome in childhood. Int $\mathrm{J}$ Obes (Lond), 2011. 35(1): p. 16-28.

222. Pittler, M.H. and E. Ernst, Complementary therapies for reducing body weight: a systematic review. Int J Obes (Lond), 2005. 29(9): p. 1030-8. 
223. Telama, R., Tracking of physical activity from childhood to adulthood: a review. Obes Facts, 2009. 2(3): p. 187-95.

224. Pan, L., et al., The association of obesity and school absenteeism attributed to illness or injury among adolescents in the United States, 2009. J Adolesc Health, 2013. 52(1): p. 64-9.

225. Timmons, B.W., et al., Systematic review of physical activity and health in the early years (aged 0-4 years). Appl Physiol Nutr Metab, 2012. 37(4): p. 773-92.

226. Kibbe, D.L., et al., Ten Years of TAKE 10!((R)): Integrating physical activity with academic concepts in elementary school classrooms. Prev Med, 2011. 52 Suppl 1: p. S43-50.

227. Troiano, R.P., et al., Physical activity in the United States measured by accelerometer. Med Sci Sports Exerc, 2008. 40(1): p. 181-8.

228. Nieman, P., et al., Psychosocial aspects of child and adolescent obesity. Paediatr Child Health, 2012. 17(4): p. 205-8.

229. Hatano, D., et al., Effect of exercise training on the density of endothelial cells in the white adipose tissue of rats. Scand J Med Sci Sports, 2011. 21(6): p. e115-21.

230. Schroeder, M., et al., Post-weaning voluntary exercise exerts long-term moderation of adiposity in males but not in females in an animal model of early-onset obesity. Horm Behav, 2010. 57(4-5): p. 496-505.

231. Gollisch, K.S., et al., Effects of exercise training on subcutaneous and visceral adipose tissue in normal- and high-fat diet-fed rats. Am J Physiol Endocrinol Metab, 2009. 297(2): p. E495-504.

232. Bouchard, C., et al., The response to long-term overfeeding in identical twins. N Engl J Med, 1990. 322(21): p. 1477-82. 
233. Vickers, S.P., H.C. Jackson, and S.C. Cheetham, The utility of animal models to evaluate novel anti-obesity agents. Br J Pharmacol, 2011. 164(4): p. 1248-62.

234. Chumlea, W.C., et al., Changes in adipocyte cellularity in children ten to 18 years of age. Int J Obes, 1982. 6(4): p. 383-9.

235. Bluher, M., The distinction of metabolically 'healthy' from 'unhealthy' obese individuals. Curr Opin Lipidol, 2010. 21(1): p. 38-43.

236. Singh, P., et al., Effects of weight gain and weight loss on regional fat distribution. Am J Clin Nutr, 2012. 96(2): p. 229-33.

237. Guh, D.P., et al., The incidence of co-morbidities related to obesity and overweight: a systematic review and meta-analysis. BMC Public Health, 2009. 9: p. 88.

238. Despres, J.P. and I. Lemieux, Abdominal obesity and metabolic syndrome. Nature, 2006. 444(7121): p. 881-7.

239. Ferrante, A.W., Jr., Obesity-induced inflammation: a metabolic dialogue in the language of inflammation. J Intern Med, 2007. 262(4): p. 408-14.

240. Smorlesi, A., et al., The adipose organ: white-brown adipocyte plasticity and metabolic inflammation. Obes Rev, 2012. 13 Suppl 2: p. 83-96.

241. Trayhurn, P. and I.S. Wood, Signalling role of adipose tissue: adipokines and inflammation in obesity. Biochem Soc Trans, 2005. 33(Pt 5): p. 107881.

242. Kubo, Y., et al., Organization of extracellular matrix components during differentiation of adipocytes in long-term culture. In Vitro Cell Dev Biol Anim, 2000. 36(1): p. 38-44.

243. Mariman, E.C. and P. Wang, Adipocyte extracellular matrix composition, dynamics and role in obesity. Cell Mol Life Sci, 2010. 67(8): p. 1277-92. 
244. Nakajima, I., et al., Extracellular matrix development during differentiation into adipocytes with a unique increase in type V and VI collagen. Biol Cell, 2002. 94(3): p. 197-203.

245. Cinti, S., et al., Adipocyte death defines macrophage localization and function in adipose tissue of obese mice and humans. J Lipid Res, 2005. 46(11): p. 2347-55.

246. Tam, C.S., et al., Adipose tissue remodeling in children: the link between collagen deposition and age-related adipocyte growth. J Clin Endocrinol Metab, 2012. 97(4): p. 1320-7.

247. Hughes, K.A., S.P. Webster, and B.R. Walker, 11-Beta-hydroxysteroid dehydrogenase type 1 (11beta-HSD1) inhibitors in type 2 diabetes mellitus and obesity. Expert Opin Investig Drugs, 2008. 17(4): p. 481-96.

248. Pinheiro, C.H., et al., Exercise prevents cardiometabolic alterations induced by chronic use of glucocorticoids. Arq Bras Cardiol, 2009. 93(4): p. $400-8,392-400$.

249. Rajia, S., H. Chen, and M.J. Morris, Voluntary post weaning exercise restores metabolic homeostasis in offspring of obese rats. Nutr Metab Cardiovasc Dis, 2012.

250. Coutinho, A.E., et al., Effect of voluntary exercise on peripheral tissue glucocorticoid receptor content and the expression and activity of 11 betaHSD1 in the Syrian hamster. J Appl Physiol, 2006. 100(5): p. 1483-8.

251. Laue, L., et al., The antiglucocorticoid and antiprogestin steroid $R U 486$ : its glucocorticoid agonist effect is inadequate to prevent adrenal insufficiency in primates. J Clin Endocrinol Metab, 1988. 67(3): p. 602-6.

252. Havel, P.J., et al., Predominately glucocorticoid agonist actions of RU-486 in young specific-pathogen-free Zucker rats. Am J Physiol, 1996. 271(3 Pt 2): p. R710-7. 
253. Stelmanska, E. and J. Swierczynski, Up-regulation of lipogenic enzyme genes expression in inguinal white adipose tissue of female rats by progesterone. J Steroid Biochem Mol Biol, 2013. 134: p. 37-44.

254. Efrat, M., S. Tepper, and R.Z. Birk, From fat cell biology to public health preventive strategies - pinpointing the critical period for obesity prevention. J Pediatr Endocrinol Metab, 2013: p. 1-13.

255. Ibanez, L., et al., Low body adiposity and high leptinemia in breast-fed infants born small-for-gestational-age. J Pediatr, 2010. 156(1): p. 145-7.

256. Ibanez, L., et al., Early development of adiposity and insulin resistance after catch-up weight gain in small-for-gestational-age children. J Clin Endocrinol Metab, 2006. 91(6): p. 2153-8.

257. Ibanez, L., et al., Visceral adiposity without overweight in children born small for gestational age. J Clin Endocrinol Metab, 2008. 93(6): p. 207983.

258. Tchernof, A. and J.P. Despres, Pathophysiology of human visceral obesity: an update. Physiol Rev, 2013. 93(1): p. 359-404.

259. Hodson, L. and K.N. Frayn, Hepatic fatty acid partitioning. Curr Opin Lipidol, 2011. 22(3): p. 216-24.

260. Rector, R.S., et al., Cessation of daily exercise dramatically alters precursors of hepatic steatosis in Otsuka Long-Evans Tokushima Fatty (OLETF) rats. J Physiol, 2008. 586(Pt 17): p. 4241-9.

261. Pouteau, E., et al., Dynamics of adipose tissue development by $2 \mathrm{H} 2 \mathrm{O}$ labeling. Methods Mol Biol, 2009. 579: p. 337-58.

262. Kajita, K., et al., Pioglitazone enhances small-sized adipocyte proliferation in subcutaneous adipose tissue. Endocr J, 2012. 59(12): p. 1107-14. 


\section{APPENDIX A}

RUN versus WL RNA-Sequencing analysis:

top up- and down-regulated transcripts 
TABLE A1. 73 Up-regulated transcripts from RNA-Seq analysis.

\section{NAME OF TRANSCRIPT}

regulatory factor X, 4 (influences HLA class II expression) gastric inhibitory polypeptide receptor

Kv channel interacting protein 1

calmodulin-like 3

SIX homeobox 4

alcohol dehydrogenase, iron containing, 1

uncoupling protein 3

cytochrome P450, family 4, subfamily F, polypeptide 22

carboxylesterase 5

growth hormone regulated TBC protein 1

reticulon 4

carrier family 25 , member 35

calcium/calmodulin-dependent protein kinase II beta

purinergic receptor P2X, ligand-gated ion channel, 5

ciliary neurotrophic factor

1-acylglycerol-3-phosphate O-acyltransferase 2

cysteine conjugate-beta lyase 2

carbonic anhydrase 3

androgen-induced 1

diazepam binding inhibitor

branched chain amino-acid transaminase 2, mitochondrial

protein phosphatase 1 , regulatory (inhibitor) subunit $1 \mathrm{~A}$

fatty acid binding protein 4 , adipocyte

acyl-CoA oxidase 1 , palmitoyl

angiotensin I converting enzyme (peptidyl-dipeptidase A) 2

phosphoserine phosphatase

phospholipase $\mathrm{A} 2$, group XVI

CD300 molecule-like family member $\mathrm{g}$

3-oxoacid CoA transferase 1

cysteine dioxygenase, type I

up-regulated during skeletal muscle growth 5

adipogenin

s family with sequence similarity 73 , member $B$

glutathione transferase zeta 1

succinate receptor 1

$\mathrm{N}$-acetyltransferase 8-like

1-acylglycerol-3-phosphate 0 -acyltransferase 9

cytochrome b5 reductase 1

adrenoceptor beta 3

MACRO domain containing 1

pleckstrin homology domain containing, family B (evectins)

member 1

asparagine synthetase

enoyl-CoA delta isomerase 1

UFM1-specific peptidase 1

acyl-CoA thioesterase 13

BRCA1-associated protein

glutathione S-transferase alpha 5

cell death-inducing DFFA-like effector $\mathrm{c}$

carboxymethylenebutenolidase

MPV17 mitochondrial membrane protein-like

3-hydroxy-3-methylglutaryl-CoA synthase 1

ubiquinol-cytochrome $\mathrm{c}$ reductase, complex III subunit XI

cytochrome c oxidase, subunit VIc-like

chemokine (C-C motif) ligand 27

pyruvate dehyrogenase phosphatase catalytic subunit 2

protein-O-mannosyltransferase 2

neuregulin 4

chromosome 15 open reading frame 61

heat shock 10kDa protein 1 (chaperonin 10)

RAN guanine nucleotide release factor

\begin{tabular}{|c|c|c|c|c|}
\hline SYMBOL & $\begin{array}{c}\text { RUN RPKM } \\
\text { AVERAGE }\end{array}$ & $\begin{array}{l}\text { WL RPKM } \\
\text { AVERAGE }\end{array}$ & $\underline{R U N / W L}$ & P-VALUE \\
\hline$(\mathrm{Rf} \times 4)$ & 2.436 & 0.761 & 3.202 & 0.0004 \\
\hline (Gipr) & 2.094 & 0.768 & 2.725 & 0.0017 \\
\hline (Kcnip1) & 15.105 & 6.149 & 2.456 & 0.0005 \\
\hline (Calml3) & 8.972 & 3.803 & 2.359 & 0.0018 \\
\hline (Six4) & 2.459 & 1.070 & 2.290 & 0.0002 \\
\hline (Adhfe1) & 49.273 & 21.536 & 2.288 & 0.0003 \\
\hline (Ucp3) & 25.046 & 11.802 & 2.122 & 0.0018 \\
\hline (Сур4f22) & 16.684 & 8.072 & 2.067 & 0.0028 \\
\hline (Ces2) & 2.369 & 1.186 & 1.997 & 0.0005 \\
\hline (Grpt1) & 1.861 & 0.932 & 1.996 & 0.0036 \\
\hline (Rtn4) & 270.074 & 138.891 & 1.945 & 0.0016 \\
\hline (Slc25a35) & 43.719 & 22.546 & 1.939 & 0.0018 \\
\hline (Camk2b) & 7.131 & 3.712 & 1.921 & 0.0041 \\
\hline (P2rx5) & 24.384 & 12.746 & 1.913 & 0.0007 \\
\hline (Cntf) & 5.948 & 3.157 & 1.884 & 0.0002 \\
\hline (Agpat2) & 394.366 & 219.378 & 1.798 & 0.0024 \\
\hline (Ccbl2) & 21.503 & 11.995 & 1.793 & 0.0004 \\
\hline (Ca3) & 1326.502 & 741.588 & 1.789 & 0.0008 \\
\hline (Aig1) & 72.044 & 40.701 & 1.770 & 0.0007 \\
\hline (Dbi) & 5.247 & 2.969 & 1.767 & 0.0022 \\
\hline (Bcat2) & 97.259 & 55.714 & 1.746 & 0.0003 \\
\hline (Ppp1r1a) & 61.494 & 35.251 & 1.744 & 0.0001 \\
\hline (Fabp4) & 18261.061 & 10506.097 & 1.738 & 0.0006 \\
\hline (Acox1) & 163.670 & 94.242 & 1.737 & 0.0004 \\
\hline (Ace2) & 1.668 & 0.967 & 1.725 & 0.0049 \\
\hline (Psph) & 28.171 & 16.389 & 1.719 & 0.0021 \\
\hline (Pla2g16) & 1129.234 & 658.953 & 1.714 & 0.0006 \\
\hline (Cd300lg) & 5.119 & 2.995 & 1.709 & 0.0016 \\
\hline (Oxct1) & 183.745 & 108.171 & 1.699 & 0.0002 \\
\hline (Cdo1) & 1446.001 & 856.056 & 1.689 & 0.0000 \\
\hline (Usmg5) & 5.843 & 3.470 & 1.684 & 0.0010 \\
\hline (Adig) & 741.757 & 440.576 & 1.684 & 0.0050 \\
\hline (Fam73b) & 2.460 & 1.461 & 1.683 & 0.0033 \\
\hline (Gstz1) & 61.024 & 36.291 & 1.682 & 0.0031 \\
\hline (Sucnr1) & 48.798 & 29.033 & 1.681 & 0.0049 \\
\hline (Nat8I) & 54.036 & 32.259 & 1.675 & 0.0014 \\
\hline (Agpat9) & 58.907 & 35.180 & 1.674 & 0.0000 \\
\hline (Cyb5r1) & 320.072 & 192.195 & 1.665 & 0.0032 \\
\hline (Adrb3) & 93.412 & 56.269 & 1.660 & 0.0048 \\
\hline (Macrod1) & 29.901 & 18.118 & 1.650 & 0.0008 \\
\hline (Plekhb1) & 8.915 & 5.425 & 1.643 & 0.0008 \\
\hline (Asns) & 64.760 & 39.666 & 1.633 & 0.0033 \\
\hline (Eci1) & 86.291 & 52.892 & 1.631 & 0.0006 \\
\hline (Ufsp1) & 5.916 & 3.635 & 1.627 & 0.0036 \\
\hline (Acot13) & 17.082 & 10.513 & 1.625 & 0.0012 \\
\hline (Brap2) & 1.856 & 1.155 & 1.608 & 0.0001 \\
\hline (Gsat5) & 2.223 & 1.384 & 1.606 & 0.0003 \\
\hline (Cidec) & 979.814 & 615.201 & 1.593 & 0.0002 \\
\hline (Cmbl) & 97.351 & 61.228 & 1.590 & 0.0009 \\
\hline (Mpv17l) & 3.451 & 2.176 & 1.586 & 0.0005 \\
\hline (Hmgcs1) & 62.952 & 39.811 & 1.581 & 0.0007 \\
\hline (Uqcr11) & 8.074 & 5.117 & 1.578 & 0.0030 \\
\hline (Cox6c) & 6.453 & 4.100 & 1.574 & 0.0000 \\
\hline$(\mathrm{Ccl} 27)$ & 1.753 & 1.116 & 1.571 & 0.0007 \\
\hline (Pdp2) & 10.458 & 6.667 & 1.569 & 0.0014 \\
\hline (Pomt2) & 24.033 & 15.359 & 1.565 & 0.0013 \\
\hline (Nrg4) & 23.397 & 14.978 & 1.562 & 0.0005 \\
\hline (C15orf61) & 14.544 & 9.382 & 1.550 & 0.0032 \\
\hline (Hspe1) & 223.989 & 144.888 & 1.546 & 0.0023 \\
\hline (Rangfr) & 24.043 & 15.553 & 1.546 & 0.0009 \\
\hline
\end{tabular}


coiled-coil-helix-coiled-coil-helix domain containing 3

ATP synthase, $\mathrm{H}+$ transporting, mitochondrial Fo complex,

subunit C3

CD36 molecule (thrombospondin receptor)

coiled-coil-helix-coiled-coil-helix domain containing 2

potassium voltage-gated channel, subfamily $\mathrm{H}$, member 2

cysteine sulfinic acid decarboxylase

thyroid hormone receptor alpha

DnaJ (Hsp40) homolog, subfamily C, member 15

phytanoyl-CoA 2-hydroxylase

quinoid dihydropteridine reductase

S100 calcium binding protein A1

protein tyrosine phosphatase, receptor type, $R$

fat storage-inducing transmembrane protein 2

$\begin{array}{ccccc}\text { (Chchd3) } & 6.915 & 4.483 & 1.543 & 0.0003 \\ & & & & \\ \text { (Atp5g3) } & 91.714 & 59.593 & 1.539 & 0.0016 \\ \text { (Cd36) } & 634.178 & 412.685 & 1.537 & 0.0001 \\ \text { (Chchd2) } & 657.043 & 428.463 & 1.533 & 0.0008 \\ \text { (Kcnh2) } & 18.340 & 11.967 & 1.533 & 0.0016 \\ \text { (Csad) } & 57.754 & 37.770 & 1.529 & 0.0012 \\ \text { (Thra) } & 1.298 & 0.851 & 1.526 & 0.0003 \\ \text { (Dnajc15) } & 66.949 & 44.377 & 1.509 & 0.0004 \\ \text { (Phyh) } & 409.641 & 271.633 & 1.508 & 0.0003 \\ \text { (Qdpr) } & 152.631 & 101.218 & 1.508 & 0.0012 \\ \text { (S100a1) } & 17.345 & 11.527 & 1.505 & 0.0045 \\ \text { (Ptprr) } & 2.774 & 1.844 & 1.504 & 0.0029 \\ \text { (Fitm2) } & 57.298 & 38.184 & 1.501 & 0.0021\end{array}$


TABLE A2. 44 Down-regulated transcripts from RNA-Seq analysis

\author{
NAME OF TRANSCRIPT \\ kinesin family member $18 \mathrm{~B}$ \\ budding uninhibited by benzimidazoles 1 homolog beta \\ ubiquitin-conjugating enzyme E2C \\ cytoskeleton associated protein 2-like \\ protein regulator of cytokinesis 1 \\ cyclin-dependent kinase 1 \\ family with sequence similarity 64 , member $A$ \\ kinesin family member $2 \mathrm{C}$ \\ kinesin family member $20 B$ \\ trophinin associated protein (tastin) \\ diaphanous homolog 3 \\ polo-like kinase 4 \\ centromere protein $\mathrm{E}, 312 \mathrm{kDa}$ \\ non-SMC condensin I complex, subunit G \\ centromere protein $\mathrm{M}$ \\ microfibrillar-associated protein 4 \\ piezo-type mechanosensitive ion channel component 2 \\ chromatin licensing and DNA replication factor 1 \\ inner centromere protein \\ thyroid hormone receptor interactor 13 \\ forkhead box $\mathrm{M} 1$ \\ v-erb-b2 erythroblastic leukemia viral oncogene homolog 3 \\ transcription factor 19 \\ thrombospondin 3 \\ vestigial like 3 \\ Rho GTPase activating protein $11 \mathrm{~A}$ \\ chromatin assembly factor 1 , subunit $A$ (p150) \\ neuron navigator 2 \\ tensin 3 \\ kin of IRRE like \\ asporin \\ zinc finger protein 367 \\ POU class 2 homeobox 2 \\ major histocompatibility complex, class I, H \\ structural maintenance of chromosomes 2 \\ RCAN family member 3 \\ cathepsin $\mathrm{K}$ \\ cyclin-dependent kinase 6 \\ placenta-specific 9 \\ drebrin 1 \\ outer dense fiber of sperm tails 2-like \\ UDP-GIcNAc:betaGal beta-1,3-N- \\ acetylglucosaminyltransferase 9 \\ ring finger and WD repeat domain 3 \\ eukaryotic translation initiation factor $2 \mathrm{C}, 2$
}

\begin{tabular}{|c|c|c|c|c|}
\hline SYMBOL & $\begin{array}{c}\text { RUN RPKM } \\
\text { AVERAGE }\end{array}$ & $\begin{array}{l}\text { WL RPKM } \\
\text { AVERAGE }\end{array}$ & RUN/WL & P-VALUE \\
\hline (Kif18b) & 0.217 & 1.519 & -6.997 & 0.0025 \\
\hline (Bub1b) & 0.316 & 2.162 & -6.831 & 0.0036 \\
\hline (Ube2c) & 1.097 & 7.307 & -6.660 & 0.0035 \\
\hline (ckap2I) & 0.266 & 1.721 & -6.479 & 0.0016 \\
\hline (Prc1) & 1.583 & 9.570 & -6.044 & 0.0012 \\
\hline (Cdk1) & 1.559 & 9.152 & -5.869 & 0.0047 \\
\hline (Fam64a) & 0.445 & 2.557 & -5.741 & 0.0015 \\
\hline (Kif2c) & 0.226 & 1.223 & -5.408 & 0.0019 \\
\hline (Kif20b) & 0.251 & 1.277 & -5.078 & 0.0021 \\
\hline (Troap) & 0.300 & 1.496 & -4.979 & 0.0029 \\
\hline (Diaph3) & 0.349 & 1.644 & -4.709 & 0.0007 \\
\hline (Plk4) & 0.446 & 1.983 & -4.442 & 0.0044 \\
\hline (Cenpe) & 0.380 & 1.633 & -4.293 & 0.0014 \\
\hline (Ncapg) & 0.588 & 2.110 & -3.589 & 0.0013 \\
\hline (Cenpm) & 0.508 & 1.682 & -3.310 & 0.0036 \\
\hline (Mfap4) & 16.300 & 53.474 & -3.281 & 0.0018 \\
\hline (Piezo2) & 0.392 & 1.260 & -3.211 & 0.0031 \\
\hline (Cdt1) & 0.604 & 1.900 & -3.146 & 0.0038 \\
\hline (Incenp) & 1.062 & 3.113 & -2.931 & 0.0011 \\
\hline (Trip13) & 0.435 & 1.249 & -2.874 & 0.0015 \\
\hline (Foxm1) & 0.804 & 2.225 & -2.766 & 0.0028 \\
\hline (Erbb3) & 1.613 & 3.977 & -2.465 & 0.0030 \\
\hline (Tcf19) & 4.152 & 9.333 & -2.248 & 0.0048 \\
\hline (Thbs3) & 3.450 & 7.676 & -2.225 & 0.0026 \\
\hline (Vg\|3) & 6.646 & 14.263 & -2.146 & 0.0013 \\
\hline (Arhgap11a) & 2.927 & 6.205 & -2.120 & 0.0009 \\
\hline (Chaf1a) & 1.395 & 2.868 & -2.056 & 0.0003 \\
\hline (Nav2) & 0.618 & 1.175 & -1.902 & 0.0034 \\
\hline (Tns3) & 3.181 & 5.873 & -1.847 & 0.0006 \\
\hline (Kirrel) & 7.471 & 13.514 & -1.809 & 0.0003 \\
\hline (Aspn) & 20.547 & 37.142 & -1.808 & 0.0026 \\
\hline (Znf367) & 2.260 & 4.080 & -1.805 & 0.0017 \\
\hline (Pou2f2) & 0.495 & 0.865 & -1.746 & 0.0036 \\
\hline (Hla-h) & 18.643 & 32.440 & -1.740 & 0.0015 \\
\hline (Smc2) & 4.259 & 7.147 & -1.678 & 0.0033 \\
\hline (Rcan3) & 2.857 & 4.757 & -1.665 & 0.0020 \\
\hline (Ctsk) & 19.441 & 31.855 & -1.639 & 0.0033 \\
\hline (Cdk6) & 3.934 & 6.325 & -1.608 & 0.0040 \\
\hline (Plac9) & 29.429 & 47.112 & -1.601 & 0.0026 \\
\hline (Dbn1) & 5.341 & 8.502 & -1.592 & 0.0037 \\
\hline (Odf2I) & 1.387 & 2.159 & -1.556 & 0.0011 \\
\hline (B2gnt9) & 4.071 & 6.239 & -1.533 & 0.0029 \\
\hline (Rfwd3) & 2.366 & 3.573 & -1.510 & 0.0009 \\
\hline (Eif2c2) & 2.501 & 3.769 & -1.507 & 0.0006 \\
\hline
\end{tabular}


Table A3. Top up-regulated IPA pathways for RUN vs. WL

Carbohydrate Metabolism, Cellular Function and Maintenance, Small Molecule Biochemistry (Score: 59 )

Acox1, Adrb3, Agpat2, Agpat9, Aig1, Asns, Bcat2, Ca3, Cd36, Chchd2, Cntf, Dbi, Fabp4, Gsta5, Hspe1, Kcnh2, Nrg4, Oxct1, Pla2g16, Ppp1r1a, Ptprr, S100a1, Sucnr1, Thra

Developmental Disorder, Hereditary Disorder, Neurological Disease (Score: 28)

Acot13, Ccbl2, Cmbl, Cyb5r1, Cyp4f22, Eci1, Fitm2, Grtp1, Pdp2, Pomt2, Qdpr, Rangrf, Usmg5

DNA Replication, Recombination, and Repair, Nucleic Acid Metabolism, Small Molecule Biochemistry (Score: 25)

Ace2, Adig, Atp5g3, Calml3, Chchd3, Cidec, Csad, Dbi, Hmgcs1, Mpv17I, Plekhb1, Psph, Six4

Molecular Transport, Amino Acid Metabolism, Lipid Metabolism (Score: 21)

Adhfe1, Cam2b, Cnajc15, Gipr, Kcnip1, Macrod1, Nat8I, P2rx5, Phyh, Rtn4, Ucp3

Table A4. Top down-regulated IPA pathways for RUN vs. WL

Cell Cycle, Cellular Assembly and Organization, DNA Replication, Recombination, and Repair (Score: 55)

Bub1b, Cdk1, Cdk6, Cdt1, Cenpe, Chaf1a, Ctsk, Dbn1, Eif2c2, Erbb3, Foxm1, Incenp, Kif20b, Kif2c, Ncapg, Odf2l, Plk4, Prc1, Smc2, Tns3, Trip13, Ube2c

Cancer, Cellular Development,Infectious Disease (Score: 40)

Arhgap11a, B3gnt9, Cenpe, Cenpm, Ckap2l, Fam64a, H2-t24, Incenp, Kif18b, Kirrel, Pou2f2, Rcan3, Rfwd3, Tcf19, Troap 
Table A5. 14 transcripts that are expressed in RUN but not WL

\section{NAME OF TRANSCRIPT}

DEAD (Asp-Glu-Ala-Asp) box polypeptide 3, X-linked glycosylation dependent cell adhesion molecule 1 homeobox A9

macrophage receptor with collagenous structure uroplakin $1 \mathrm{~A}$

5S RNA

casein alpha s2-like $A$

synaptophysin-like 2

xin actin-binding repeat containing 2

ankyrin repeat domain 23

glucocorticoid induced transcript 1

leiomodin 3

popeye domain containing 3

epithelial cell adhesion molecule

\begin{tabular}{|c|c|}
\hline SYMBOL & $\begin{array}{l}\text { RPKM } \\
\text { VALUE }\end{array}$ \\
\hline$(D d x 3 x)$ & 18.005 \\
\hline (Glycam1) & 9.086 \\
\hline (Hoxa9) & 6.562 \\
\hline (Marco) & 5.820 \\
\hline (Upk1a) & 2.741 \\
\hline (Rna5s3) & 2.641 \\
\hline (Csn1s2a) & 2.253 \\
\hline (Sypl2) & 2.221 \\
\hline (Xirp2) & 2.078 \\
\hline (Ankrd23) & 1.238 \\
\hline (Glcci1) & 1.168 \\
\hline$(\operatorname{Lmod} 3)$ & 1.258 \\
\hline (Popdc3) & 1.099 \\
\hline (Epcam) & 1.054 \\
\hline
\end{tabular}


Table A6. 32 transcripts that are expressed in WL but not RUN

\section{NAME OF TRANSCRIPT}

carboxypeptidase B1

phospholipase A2, group IB (PLA2G1B)

protease, serine, 3 (Prss3)

colipase, pancreatic (CLPS)

prepronociceptin (PNOC)

pancreatic lipase-related protein 1 (Pnliprp1)

syncollin (SYCN)

protease, serine, 2 (trypsin 2) (PRSS2)

BARX homeobox 1 (Barx1)

C2 calcium-dependent domain containing 4D (C2cd4d)

serine peptidase inhibitor, Kazal type 1 (SPINK1)

norvegicus glycerophosphodiester phosphodiesterase domain containing 2 (Gdpd2)

D4, zinc and double PHD fingers family 1 (DPF1)

angiotensin II receptor, type 2 (AGTR2)

serine (or cysteine) peptidase inhibitor, clade I, member 2

(Serpini2)

synaptosomal-associated protein, 25kDa (SNAP25)

protein disulfide isomerase family $A$, member 2 (Pdia2)

runt-related transcription factor 1 ; translocated to, 1 (cyclin $D$ -

related) (RUNX1T1)

geminin coiled-coil domain containing (GMNC)

endoplasmic reticulum protein 27 (Erp27)

chemokine ( $\mathrm{C}-\mathrm{C}$ motif) receptor 3 (Ccr3)

retinol binding protein 2, cellular (RBP2

sodium channel, voltage-gated, type III, alpha subunit (SCN3A)

SLIT and NTRK-like family, member 2 (Slitrk2)

family with sequence similarity 83 , member D (Fam83d)

\begin{tabular}{cc} 
& RPKM \\
SYMBOL & VALUE \\
\hline (Cpb1) & 114.338 \\
(Pla2g1b) & 70.900 \\
(Prss3) & 65.286 \\
(Clps) & 53.700 \\
(Pnoc) & 41.326 \\
(Pnliprp1) & 34.388 \\
(Sync) & 30.699 \\
(Prss2) & 12.454 \\
(Barx1) & 11.817 \\
(C2cd4d) & 5.089 \\
(Spink1) & 3.689 \\
& \\
(Gdpd2) & 2.619 \\
(Dpf1) & 2.044 \\
(Agtr2) & 1.885 \\
& \\
(Serpini2) & 1.635 \\
(Snap25) & 1.620 \\
(Pdia2) & 1.614 \\
& \\
(Runx1t1) & 1.388 \\
(Gmnc) & 1.388 \\
(Erp27) & 1.221 \\
(Ccr3) & 1.209 \\
(Rbp2) & 1.200 \\
(Scn3a) & 1.134 \\
(Slitrk2) & 1.047 \\
(Fam83d) & 1.016 \\
& \\
&
\end{tabular}




\section{APPENDIX B}

Epicardial fat gene expression after aerobic exercise training in pigs with coronary atherosclerosis: relationship to visceral and subcutaneous fat

Published in J Appl Physiol 109: 1904-1912, 2012 


\title{
Epicardial fat gene expression after aerobic exercise training in pigs with coronary atherosclerosis: relationship to visceral and subcutaneous fat
}

\author{
Joseph M. Company, ${ }^{1}$ Frank W. Booth, ${ }^{1,2,3}$ M. Harold Laughlin, ${ }^{1,2,3}$ Arturo A. Arce-Esquivel, ${ }^{1}$ \\ Harold S. Sacks, ${ }^{4}$ Suleiman W. Bahouth, ${ }^{5}$ and John N. Fain ${ }^{6}$ \\ ${ }^{1}$ Department of Biomedical Sciences, College of Veterinary Medicine, University of Missouri, Columbia; ${ }^{2}$ Dalton \\ Cardiovascular Research Center, University of Missouri, Columbia; ${ }^{3}$ Department of Medical Pharmacology and Physiology, \\ College of Medicine, University of Missouri, Columbia, Missouri; and Departments of ${ }^{4}$ Medicine, ${ }^{5}$ Pharmacology, and \\ ${ }^{6}$ Molecular Sciences, College of Medicine, University of Tennessee Health Science Center, Memphis, Tennessee. \\ Submitted 4 June 2010; accepted in final form 7 October 2010
}

Company JM, Booth FW, Laughlin MH, Arce-Esquivel AA, Sacks HS, Bahouth SW, Fain JN. Epicardial fat gene expression after aerobic exercise training in pigs with coronary atherosclerosis: relationship to visceral and subcutaneous fat. J Appl Physiol 109: 1904-1912, 2010. First published October 14, 2010; doi:10.1152/japplphysiol.00621.2010.-Epicardial adipose tissue (EAT) is contiguous with coronary arteries and myocardium and potentially may play a role in coronary atherosclerosis (CAD). Exercise is known to improve cardiovascular disease risk factors. The purpose of this study was to investigate the effect of aerobic exercise training on the expression of 18 genes, measured by RT-PCR and selected for their role in chronic inflammation, oxidative stress, and adipocyte metabolism, in peri-coronary epicardial (cEAT), peri-myocardial epicardial (mEAT), visceral abdominal (VAT), and subcutaneous (SAT) adipose tissues from a castrate male pig model of familial hypercholesterolemia with CAD. We tested the hypothesis that aerobic exercise training for $16 \mathrm{wk}$ would reduce the inflammatory profile of mRNAs in both components of EAT and VAT but would have little effect on SAT. Exercise increased mEAT and total heart weights. EAT and heart weights were directly correlated. Compared with sedentary pigs matched for body weight to exercised animals, aerobic exercise training reduced the inflammatory response in mEAT but not cEAT, had no effect on inflammatory genes but preferentially decreased expression of adiponectin and other adipocyte-specific genes in VAT, and had no effect in SAT except that IL-6 mRNA went down and VEGFa mRNA went up. We conclude that 1) EAT is not homogeneous in its inflammatory response to aerobic exercise training, 2) cEAT around CAD remains proinflammatory after chronic exercise, 3) cEAT and VAT share similar inflammatory expression profiles but different metabolic mRNA responses to exercise, and 4) gene expression in SAT cannot be extrapolated to VAT and heart adipose tissues in exercise intervention studies.

perivascular adipose tissue; epicardial adipose tissue; familial hypercholesterolemia

OBESITY is a cardiovascular disease (CVD) risk factor, and there is a strong association between obesity and atherosclerosis (15). Even though the link between obesity, atherosclerosis, and CVD morbidity and mortality has been widely discussed, the mechanism(s) by which these are linked is not sufficiently elucidated and remains controversial (25). Adipose tissue functions as an endocrine organ with the potential to modulate pathophysiology by producing and releasing pro- and antiinflammatory cytokines that act in a paracrine and endocrine

Address for reprint requests and other correspondence: J. M. Company, E102 Veterinary Medicine Bldg., Dept. of Biomedical Sciences, Univ. of Missouri, Columbia, MO 65211 (e-mail: jmc2gd@mizzou.edu). fashion. With the increase in obesity and obesity-related diseases, the need to clarify adipose tissue phenotype is essential.

Evidence is increasing that different adipose tissue depots have different functions. This is especially apparent in visceral and subcutaneous adipose tissue (SAT). Visceral adipose tissue (VAT) is considered to be more pathogenic than SAT, perhaps due to its proximity to metabolic organs (7). Many epidemiological studies have pointed to VAT as a risk factor for cardiovascular disease, but the mechanistic link remains partially unclear (4). Epicardial adipose tissue (EAT) shares an embryologic origin with VAT $(6,24,44)$ and is of particular interest due to its close anatomic association with coronary arteries and myocardium. EAT thickness (via echocardiographic data) strongly correlates with abdominal visceral adipose tissue (via MRI) (25), and EAT mass has been suggested to reflect intra-abdominal VAT (26). In addition, EAT is a source of proinflammatory cytokines and has been shown to have more macrophage infiltration than SAT in patients with coronary artery disease (31). Therefore, like VAT's correlation with CVD, EAT may play a role in cardiac disease by its association with increased intramyocardial triglyceride content (44) or by displaying a similar adipokine profile as VAT $(1,35)$.

EAT can arbitrarily be divided into coronary peri-vascular adipose tissue (cEAT), which is directly around or on the coronary artery adventitia, and EAT over the myocardium (mEAT) since the two components may be functionally distinct (8) despite their anatomic contiguity. cEAT may interact with vascular cells in a paracrine fashion or through vasa vasora to affect vascular function $(17,25,44,47)$. Despite data supporting AT role as an endocrine organ, little is known about the phenotype of cEAT and if/how it differs from mEAT and, in turn, how these adipose tissue pads influence coronary and cardiac function, respectively. cEAT and mEAT may provide insight into a mechanistic link between adiposity and cardiac abnormalities.

Exercise is known to improve CVD risk factors (37). While data show that exercise reduces VAT mass independent of SAT mass $(48,49)$ and reduces EAT thickness $(30)$, only a few studies investigated the molecular characteristics of VAT and SAT and no studies have investigated the effect of aerobic exercise on the molecular characteristics of cEAT or mEAT. A pig model, specifically one of familial hypercholesterolemia, was selected not only because the cardiovascular system of the pig closely approximates the human, but it also provides a good large animal model for humans (11) and hypercholesterolemia is a risk factor for human CVD (52). Human 
hypercholesterolemia is characterized by two- to threefold increases in low-density lipoprotein (LDL) cholesterol, causing rapidly progressing atherosclerosis, which leads to premature CVD (50).

The purpose of this study was to investigate the effect of 16 wk of aerobic exercise training on the expression of 18 genes selected for their role in chronic inflammation, oxidative stress, and adipocyte metabolism in four adipose tissue depots in a pig model of familial hypercholesterolemia (FH) with coronary artery disease (CAD): SAT, VAT (omental adipose tissue), cEAT, and mEAT. Given the importance of chronic inflammation in atherogenesis and the improvement of CVD risk factors with exercise, we hypothesized that aerobic exercise training will reduce the inflammatory profile of mRNAs in both components of EAT and VAT but will have little effect on SAT.

\section{METHODS}

Experimental animals. Thirteen castrated adult male FH pigs (10-11 mo of age) were used in this study. The Rapacz Familial Hypercholesterolemic (FH) model was developed at the University of Wisconsin by selective breeding $(19,21,22,33,38,39,40,41)$. These swine are characterized by a single missense mutation in the LDL receptor (LDLR) that decreases LDLR affinity for $L D L$, resulting in elevated total cholesterol levels between 180 and $240 \mathrm{mg} / \mathrm{dl}(20)$. We confirm the hypercholesterolemia of these pigs on a normal diet [total cholesterol $=360 \pm 15 \mathrm{mg} / \mathrm{dl}$, high-density lipoprotein (HDL) cholesterol $=30 \pm 1 \mathrm{mg} / \mathrm{dl}$, and total cholesterol $/ \mathrm{HDL}$ cholesterol = $12 \pm 1]$. Pigs were randomly assigned to a sedentary (SED, $n=8$ ) or aerobic exercised (EX, $n=5$ ) group. Both SED and EX were fed 800 $\mathrm{g} /$ day of the University of Wisconsin gestation diet (a cholesterol-free, $3 \%$ fat, corn-soybean-based diet) and were allowed ad libitum access to water. Pigs were housed in rooms with a 12:12-h light-dark cycle at $20-24^{\circ} \mathrm{C}$. It is a standard procedure to use castrated male pigs for exercise studies. Sex hormones can have a significant influence on adipose tissue biology. Whether castration influenced adipose tissue metabolism in this experimental pig model is unclear since no studies were done comparing fat depots in castrated animals with sex hormone-replaced animals.

Pigs underwent experimental treatment for 16-20 wk where SED pigs were restricted to their pens $(2 \times 4 \mathrm{~m})$ and $\mathrm{EX}$ pigs performed a moderate-intensity $(\sim 70 \%$ of maximum heart rate) daily aerobic exercise-training program on treadmills one time per day, 5 days/wk $(42,53,54)$. Briefly, the aerobic exercise training protocol entailed a 5-min warm up at 2-2.5 miles per hour ( $\mathrm{mph}), 15 \mathrm{~min}$ at $4 \mathrm{mph}$, and $20 \mathrm{~min}$ at $3 \mathrm{mph}$, and a $5 \mathrm{~min}$ cool-down at $2-2.5 \mathrm{mph}$. The duration and intensity were increased each week so that by week 10 of training, the pigs performed a 5 -min warm up at $2-2.5 \mathrm{mph}, 15 \mathrm{~min}$ at $6.5-7$ $\mathrm{mph}$, and $60 \mathrm{~min}$ at $4.5-5 \mathrm{mph}$, and a $5 \mathrm{~min}$ cool-down at $2-2.5 \mathrm{mph}$. The training program's effectiveness was ascertained from measurements of endurance time (pre- and post-stress testing) and heart weight/body weight ratios. The Animal Care and Use Committee at the University of Missouri approved all experimental protocols.

Tissue collection. Intramuscular ketamine/xylazine and intravenous pentothal was used to deeply anesthetize the pigs at the end of the 16to 20-wk experimental treatment, and euthanasia was achieved by heart extraction. The heart was placed in iced KREBS buffer, and cEAT and mEAT were removed from the heart surface by the same person, thereby minimizing the possibility that weight differences between the two adipose tissues were due to dissection technique differences. cEAT was defined and identified as the thick, contiguous adipose tissue, forming a ridge, and surrounding the coronary vessel extending from $\sim 10 \mathrm{~mm}$ up to $\sim 20 \mathrm{~mm}$ on each side of the vessel wall (Fig. 1). mEAT was defined and identified as the thinner contiguous adipose tissue beginning $\sim 10 \mathrm{~mm}$ to $20 \mathrm{~mm}$ away from the coronary vessel extending down on the ventricular myocardium. SAT was removed from the hip and is defined as the adipose tissue located beneath the skin but superficial to the underlying muscle. VAT was collected from omental adipose tissue interconnecting with the visceral organs in the abdominal peritoneal cavity. The adipose tissue from each depot was immediately snap-frozen in a metal beaker containing isopentane that had been sitting in liquid nitrogen long enough that it had begun to freeze. The adipose tissue was then removed from the isopentane and placed in a plastic bag sitting on dry
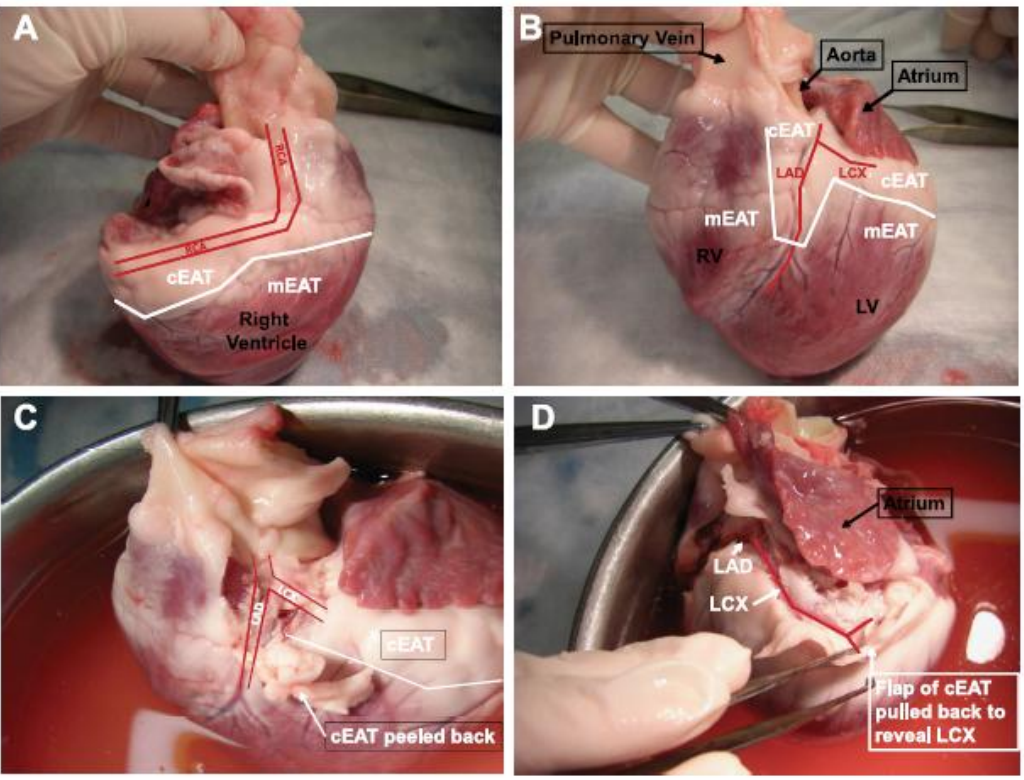

$J$ Appl Physiol • vOL. $109 \cdot$ DECEMBER $2010 \cdot$ www.jap.org
Fig. 1. Dissection procedure for coronary epicardial adipose tissue (cEAT) and myocardial epicardial adipose tissue (mEAT). A and $B$ : intact right side and left side of the heart, respectively, before dissection. The black line indicates the approximate edge between cEAT and mEAT. RCA, right coronary artery; $\mathrm{LAD}$, left anterior descending coronary vessel; LCX, left circumflex coronary vessel; RV, right ventricle, LV, left ventricle. $C$ : cEAT layer is peeled back to reveal the LAD and LCX coronary vessels. $D$ : cEAT layer is peeled back to reveal more of the LAD and LCX coronary vessel. 
ice. The bags were closed and the fat was stored in a $-80^{\circ} \mathrm{C}$ freezer until analysis. Coronary vessels were collected for immunohistochemistry. Histological assessment of atherosclerosis in coronary arteries include Sudan IV-fatty streaks (distribution of atherosclerosis) and $5-\mu \mathrm{m}$ sections were stained according to Verhoeff's method for elastin for grading of the lesion. To quantify Sudan IV staining, the percentages represent the percent of the lumen that is covered by lesions (i.e., fatty streaks) that stain positively for Sudan IV. To quantify IMT, for each pig we identified the area of the vessel with the greatest thickness (maximum IMT) and also the one with the least thickness (minimum IMT). Then, in each area we perform three separate measurements from the lumen to the external elastic lamina. Finally, we averaged those three measurements and report the maximum and minimum IMT.

mRNA isolation. Approximately $0.5 \mathrm{~g}$ of frozen tissue was homogenized with $5 \mathrm{ml}$ of a monophasic solution of phenol and guanidine isothiocyanate (TRIzol reagent, Invitrogen, Carlsbad, CA) using a Polytron homogenizer. It was assumed that the small 0.5 -g sample of fat was representative of the total mass of each fat depot. In addition, we found no evidence of a difference in the yield of total RNA per gram of tissue for cEAT vs. mEAT. The total RNA per gram values in relative units are cEAT $=416 \pm 23$ and $\mathrm{mEAT}=349 \pm 30$ as the mean \pm SE for all 13 pigs. The extracts were spun at $12,000 \mathrm{~g}$ for 10 min at $2-8^{\circ} \mathrm{C}$. A Pasteur pipette was used to remove the fat layer above the extract.

$R T-P C R$. The mRNA assay involved real-time quantitative PCR $(13,14)$. Transcriptor First Strand cDNA synthesis Kit from Roche Diagnostics was used on equal quantities of RNA to prepare the complementary DNA (cDNA). The Roche Lightcycler 480 Real-time RT-PCR system and Roche's Universal Probe Library of short hydrolysis Locked Nucleic Acid (LNA) dual hybridization probes combined with the primers recommended by their web-based assay design center [http://www.universalprobelibrary.com] were used for mRNA quantification. Integrated DNA Technologies (Coralville, IA) synthesized the primers. Eighteen mRNAs were targeted for their roles $I$ ) in inflammation: adiponectin, interleukin -1 receptor antagonist (IL1Ra), interleukin-6 (IL-6), interleukin-8/CXCL8 (IL-8), toll-like receptor 4 (TLR4), plasminogen activator inhibitor-1 (PAI-1), prostaglandin $\mathrm{D}_{2}$ synthase (PGDS), angiotensinogen, vascular endothelial growth factor a (VEGFa), and visfatin; 2) as pro-oxidants: heme oxygenase, cytochrome $c$ oxidase subunit 6 ; and as anti-oxidants: mitochondrial Mn superoxide dismutase-2 (MnSOD), endotheliumderived nitric oxide synthase (eNOS) and glutathione peroxidase 3 (GPX3); and 3) in adipocyte metabolism: fatty acid binding protein 4 (FABP4), perilipin, and uncoupling protein-2 (UCP-2). Cyclophilin was used as the "housekeeping gene"(see below).

In each assay, $55 \mathrm{ng}$ per tube of total RNA (determined by absorption at $260 \mathrm{~nm}$ in a spectrophotometer) was used, and the ratio of right to left primers was 1 for each assay. The data were obtained as crossing point values $(\mathrm{Cp})$ obtained by the second derivative maximum procedure as described by Roche Applied Science technical notes LC10/2000 and 13/2001 [http://www.roche-applied-science. com/sis/rtpcr/htc/index.jsp]. The Cp values are comparable to crossing threshold $(\mathrm{Ct})$ values as defined by $\mathrm{ABI}$ (http://www.rdml.org), and will be referred to as $\mathrm{Ct}$ values in this paper. The average value of six replicates was used for cyclophilin values in each tissue of each pig and of duplicate samples for the other mRNAs. Cyclophilin was used as the recovery standard to normalize the data, which corrected for the effects of exercise on the recovery of cyclophilin in each tissue. No correction was necessary for SAT or cEAT Ct since exercise did not affect cyclophilin $\mathrm{Ct}(-0.01 \pm 0.30$ and $0.04 \pm 0.42$, respectively). Exercise did affect cyclophilin Ct for mEAT; therefore those data were corrected by $-0.76 \pm 0.26$.

Relative quantification of the data was calculated using the comparative $\mathrm{Ct}$ method, which removes the need for standard curves. Ratios calculated from $\Delta \mathrm{Ct}$ are based on a $\log _{2}$ scale $\left(2^{-\Delta C t}\right)$. This method is the comparative $\mathrm{C}_{\mathrm{t}}$ procedure expressed in the ABI PRISM
7700 Sequence Detection System user Bulletin 2 for quantitative RT-PCR. The calculation of ratios was done without an efficiency correction by assuming that the amount of target molecules doubles with every PCR cycle.

Statistics. A two-tailed Student $t$-test was performed to determine if differences between exercise and sedentary groups were significant at a $P$ value of $<0.05$. Statistical analysis of mRNA values was based on the $\Delta \mathrm{Ct}$ values before $\log _{2}$ transformation to ratios. A between-group comparison using the means of the $\Delta \mathrm{Ct}$ values was performed to compare cEAT to the other depots

\section{RESULTS}

Experimental animals. Representative pictures from EX and SED hearts are shown in Fig. 2. mEAT mass was significantly higher in the EX pigs (Table 1). There was a nonsignificant trend $(P=0.063$ ) for total EAT mass to be higher in EX pigs. There was a significant correlation of heart weight with total EAT in all 13 pigs (adjusted $r^{2}=0.48, P=0.005$ ) (Fig. 3) and by inference of total EAT with myocardial mass since EAT accounted for only $\sim 3 \%$ of the total heart weight. Although body weight was not different between SED and EX, the effect of aerobic exercise was apparent. Heart weight, expressed in absolute terms $(\mathrm{g})$, and the heart weight/body weight ratio $(\mathrm{g} / \mathrm{kg})$ were higher in EX pigs. EX pigs showed significantly more duration of running than SED in the post-stress test $(27.70 \pm 0.13$ vs. $21.00 \pm 0.71 \mathrm{~min})$. In addition, SED pigs showed a significant decrease in running time between the preand post-stress test $(24.21 \pm 1.22$ vs. $21.00 \pm 0.71 \mathrm{~min})$. All pigs showed evidence of atherosclerosis (Fig. 4). As shown in Table 2, there was no significant difference between EX and SED IMT. Sudan IV staining revealed $9.66 \%$ in EX + SED, $14.31 \%$ in EX, and $6.6 \%$ in SED (EX vs. SED, $P=0.10$ ).

Sedentary pigs showed almost no difference in gene expression between cEAT and mEAT. We found no evidence of a difference in the yield of total RNA per gram of tissue for cEAT vs. mEAT. As shown in Fig. 5, with the exception of a $60 \%$ greater amount of cytochrome $c$ oxidase mRNA in mEAT, there was no statistically significant difference between cEAT and mEAT in the expression of any of the other 17 mRNAs examined under sedentary conditions. mEAT and cEAT differed from VAT and SAT in several respects. There was far less heme oxygenase, angiotensinogen, adiponectin, perilipin, and FABP4 in both cEAT and mEAT than in VAT or SAT. Gene expression in VAT was more comparable to that in SAT than to that in cEAT or mEAT for all genes except PGDS whose expression was elevated in VAT as well as cEAT or mEAT. Of the putative inflammatory markers, none was elevated in cEAT compared with VAT or SAT except for PGDS.

cEAT was distinctly different from $m E A T$ in response to aerobic exercise. The effect of aerobic exercise on the relative expression of mRNAs was compared in cEAT, mEAT, VAT, and SAT (Fig. 6). Peri-coronary epicardial adipose tissue (cEAT) is distinctly different in its response to aerobic exercise than peri-myocardial epicardial adipose tissue (mEAT). mRNAs are downregulated in response to aerobic exercise in mEAT but not cEAT. Of the 18 mRNAs shown in Fig. 5, it is evident from Fig. 6 that aerobic exercise reduced the expression of inflammatory genes IL1-Ra, IL-6, IL-8, PAI-1, and PGDS and redox genes eNOS and cytochrome $c$ oxidase in mEAT but not cEAT, in which superoxide dismutase was the only molecule to decrease. 


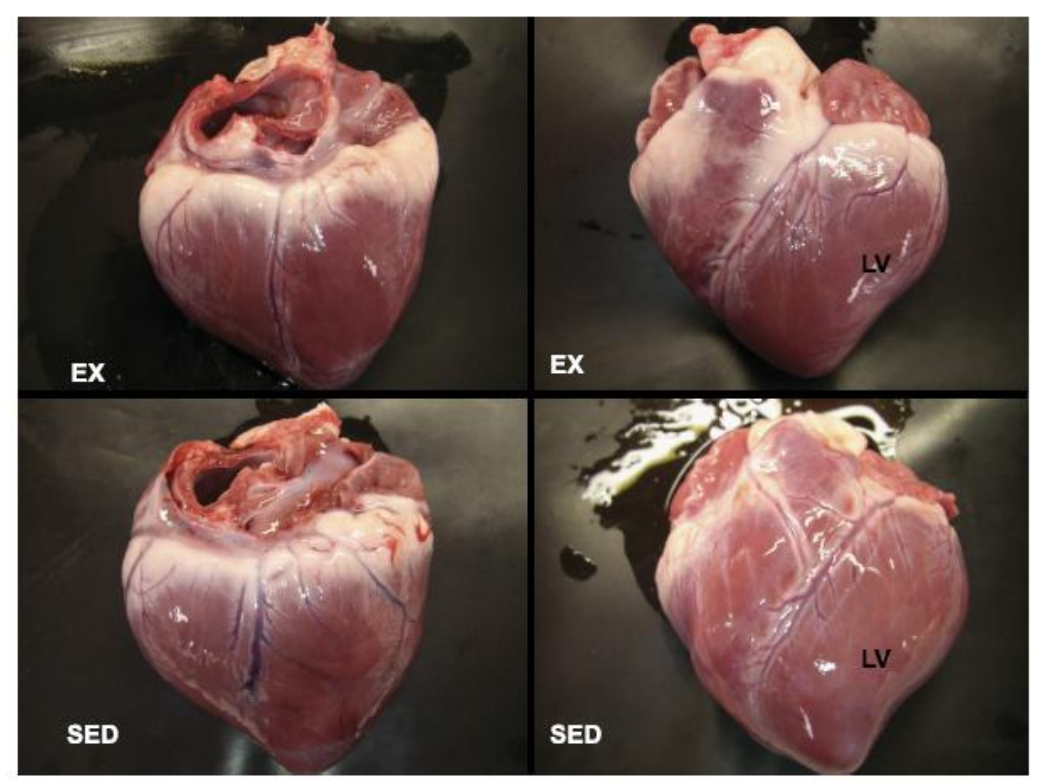

Fig. 2. Representative pictures of EX and SED hearts. EX, heart from aerobic exercisetrained familial cholesterolemia (FH) pig: SED, heart from sedentary FH pig.

VAT exhibited different mRNA expression responses to aerobic exercise training. As shown in Fig. 6, after exercise, VAT showed a striking absence of changes in inflammatory and redox gene expression and instead a downregulation of genes involved with adipocyte metabolism such as FABP4, perilipin, adiponectin, and UCP-2 that were not observed in cEAT and mEAT. Exercise had no effect on cyclophilin, eNOS, PAI-1, IL1-Ra, cytochrome $c$ oxidase, or IL-8 in VAT.

Only a limited number of genes in SAT were altered by aerobic exercise training. Of the 18 mRNAs that were measured in SAT shown in Fig. 5, only VEGFa gene expression was enhanced, and IL-6 decreased by exercise in SAT (Fig. 6). Clearly exercise affects gene expression to a far lesser extent in SAT than in VAT fat where the expression of 6 genes was reduced by exercise or mEAT where the expression of 10 genes was reduced by exercise (Fig. 6).

Table 1. Pig characteristics

\begin{tabular}{lcc}
\hline \hline & $\operatorname{SED}(n=8)$ & $\operatorname{EX~}(n=5)$ \\
\hline $\begin{array}{l}\text { EAT content } \\
\quad \text { Total peri-coronary epicardial adipose } \\
\quad \text { tissue (cEAT), }\end{array}$ & $4.10 \pm 0.36$ & $4.99 \pm 0.57$ \\
$\quad \begin{array}{l}\text { Total peri-myocardial epicardial } \\
\quad \text { adipose tissue (mEAT), g }\end{array}$ & $0.75 \pm 0.16$ & $1.55 \pm 0.30^{*}$ \\
$\quad$ Total EAT, g & $4.85 \pm 0.40$ & $6.54 \pm 0.82$ \\
Other characteristics & $169.1 \pm 4.3$ & $191.4 \pm 8.2^{*}$ \\
$\quad$ Heart weight, g & $49.4 \pm 41.6$ & $49.8 \pm 1.5$ \\
$\quad$ Body weight, kg & $3.4 \pm 0.3$ & $3.8 \pm 0.1^{*}$ \\
Heart weight/body weight ratio, g/kg & $2.9 \pm 0.2$ & $3.4 \pm 0.2$ \\
$\quad$ Heart AT \% (total EAT/heart weight), & $25.21 \pm 1.22$ & $26.54 \pm 1.53$ \\
$\quad$ Pre-stress test, min & $21.00 \pm 0.71 \dagger$ & $27.70 \pm 0.13^{*}$ \\
Post-stress test, min & &
\end{tabular}

Data are presented as means \pm SE. EAT, epicardial adipose tissue; AT, adipose tissue; SED, sedentary familial hypercholesterolemia $(\mathrm{FH})$ pig; EX, exercise-trained FH pig. ${ }^{*} P<0.05$ for SED vs. EX. $+P<0.05$ for pre-stress test vs. post-stress test.

\section{DISCUSSION}

The purpose of this study was to investigate the effects of 16 wk of aerobic exercise training on the inflammatory, oxidative stress, and metabolic mRNA phenotypes of four adipose tissue depots in a pig model of FH with CAD. The major finding that arose from the study was that aerobic exercise training increased the mass of mEAT but not cEAT at the same time as it reduced the inflammatory response in mEAT but not cEAT. These very different responses to exercise strongly suggest that these two components of EAT are functionally distinct. Other important findings were 1 ) exercise did not downregulate inflammatory and redox genes in VAT except for PGDS and superoxide dismutase, respectively, but it did lower expression of adipocyte-specific adiponectin, FABP4, and perilipin $\mathrm{mR}$ NAs, which was not observed in cEAT and mEAT; and 2) SAT did not respond to aerobic exercise training except that VEGFa mRNA went up and IL-6 mRNA went down.

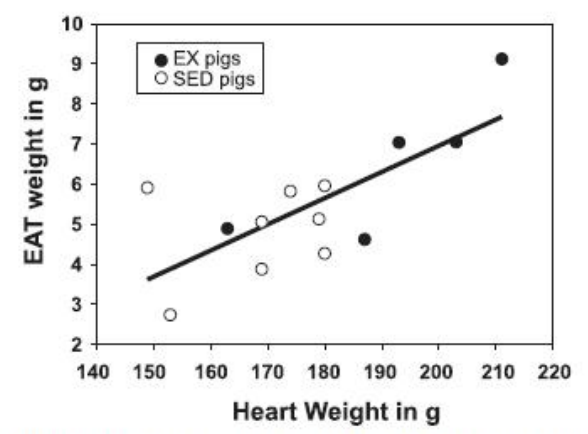

Fig. 3. Correlation of heart weight with total EAT weight for all 13 pigs. $r^{2}=$ 0.53 ; adjusted $r^{2}=0.48, P=0.005$. 
Fig. 4. Intima-media thickness (IMT): grading of lesion of structural atherosclerosis. LAD-ex, left anterior descending coronary artery from EX pig; RCA-ex, right coronary artery from EX pig; LAD-sed, left anterior descending coronary artery from SED pig; RCA-sed, right coronary artery from SED pig. The vessels are stained for VVG to identify elastin (black) and collagen (darkred; purple) fibers, which were are use to determine the IMT. Scale bar, $100 \mu \mathrm{m}$.

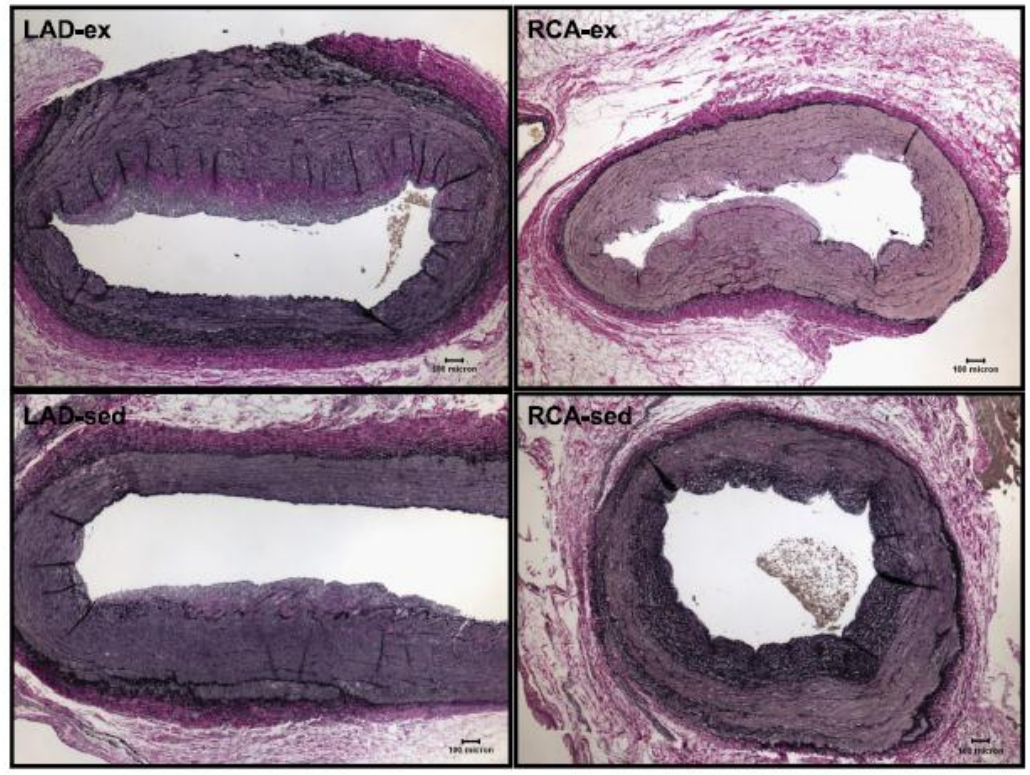

Anthropometric data. It is important to note that this was not a weight-loss study since both groups of pigs were fed the same amount of food each day and there was no body weight difference between EX and SED pigs at the end of the study. This suggested that the effects on adipose tissue phenotype were due to aerobic exercise training per se. Interestingly, despite no change in body weight, EX pigs had twice as much mEAT as SED pigs, which may be related to their $13 \%$ greater heart weight. The reason for the expansion of epicardial fat over the myocardium in concert with myocardial hypertrophy after chronic exercise in pigs is unclear. Our data showing a direct correlation between EAT and myocardial weights is in keeping with human autopsy studies, in which both left and right ventricular weight correlated directly with dissected epicardial fat weight, a relationship that suggests reciprocal regulation between the two tissues (9). The percentage of epicardial adipose tissue (expressed as total heart adipose tissue weight/heart weight) was also not altered by aerobic exercise. This finding is contrary to the findings of Kim et al. (29) who investigated the effect of exercise on epicardial adipose tissue thickness in obese, middle-aged men. These subjects underwent 12 wk of aerobic exercise training (60-70\% maximum heart rate, $60 \mathrm{~min}, 3$ days/wk). Epicardial adipose tissue thickness was measured by transthoracic echocardiography. Kim et al. (29) found a significant reduction in epicardial adipose tissue thickness. Besides the difference in species, another possible reason for the discrepancy between our stud- ies and those of Kim et al. is that the human subjects were obese, while the FH pigs were not. Another possible difference is that food intake was restricted in exercised pigs to that of sedentary pigs. Given that exercise training has been shown to reduce atherosclerosis, it is surprising that we did not see reductions in Sudan IV staining or reductions in IMT in the EX pigs; rather there was a trend for an increase in Sudan IV staining for EX pigs and no difference in IMT between EX and SED. One explanation for this counterintuitive result is that the elevated total cholesterol levels present in these FH pigs overpowers the effect of exercise in coronary arteries. Junyent et al. (28) related IMT to established and emergent risk factors in humans with FH and found that physical exercise was independently associated with IMT. Others have found similar results with exercise $(5,34)$. Mayet et al. (34) found no disparity in IMT between elite female athletes and sex-matched control subjects and attributed this finding to "intermittent exposure to markedly elevated systemic arterial pressures during exercise, resulting in intermittent elevations in carotid wall tensile stress."

Coronary peri-vascular epicardial adipose tissue was distinctly different from contiguous peri-myocardial epicardial adipose tissue in response to aerobic exercise. The novel finding in this study was that aerobic exercise training reduced a composite of 10 inflammatory and redox genes in mEAT but only one antioxidant gene, superoxide dismutase, and no proinflammatory genes in cEAT. This is contrary to our hypothesis

Table 2. Mean maximum and minimum intima-media thickness values

\begin{tabular}{lcccr}
\hline \hline & LAD-IMT Maximum, $\mu \mathrm{m}$ & RCA-IMT Maximum, $\mu \mathrm{m}$ & LAD-IMT Minimum, $\mu \mathrm{m}$ & RCA-IMT Minimum, $\mu \mathrm{m}$ \\
\hline EX & $631.4 \pm 108.3$ & $575.8 \pm 99.6$ & $208.4 \pm 31.8$ & $265.8 \pm 33.0$ \\
SED & $721.6 \pm 161.8$ & $451.1 \pm 43.8$ & $230.6 \pm 23.7$ & $228.7 \pm 24.0$
\end{tabular}

Data are presented as means \pm SE. IMT, intima-media thickness, LAD, left anterior descending coronary artery, RCA, right coronary artery. 

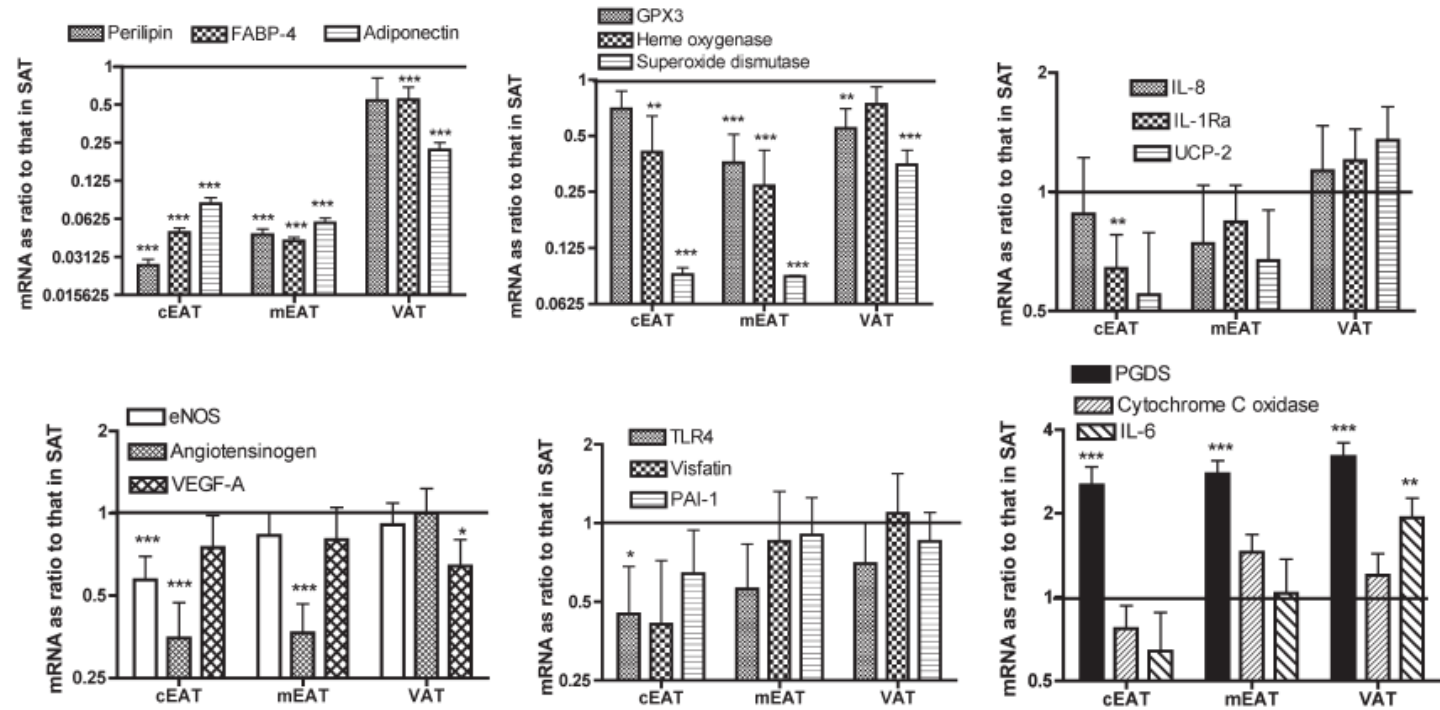

Fig. 5. Comparison of peri-coronary EAT (cEAT), peri-myocardial EAT (mEAT), and visceral omental adipose tissue (VAT) gene expression to that in subcutaneous adipose tissue (SAT) of sedentary pigs. The values are expressed as the ratio of mRNA expression in mEAT, cEAT, and VAT to that in SAT in 8 sedentary pigs. The ratios are the means $\pm \mathrm{SE}$ and derived from the $\Delta \mathrm{Ct}$ corrected for the recovery of cyclophilin. With the exception of cytochrome $c$ oxidase $(P=0.005)$, none of the mRNAs were expressed at significantly different values in cEAT vs. mEAT. FABP4, fatty acid binding protein-4; GPX3, glutathione peroxidase 3; IL-8, interleukin-8; IL-1Ra, interleukin 1 receptor antagonist; UCP-2, uncoupling protein-2; eNOS, endothelial nitric oxide synthase; VEGFa, vascular endothelial growth factor a; TLR4, toll-like receptor 4; PAI-1, plasminogen activator inhibitor 1; PGDS, prostaglandin $\mathrm{D}_{2}$ synthase; IL-6, interleukin 6. Statistically significant differences between expression in cEAT, mEAT, or VAT relative to SAT are shown as follows: ${ }^{*} P<0.05$, $* * P<0.025$, ${ }^{* * *} P<$ 6. Statistic 0.005 .

that aerobic exercise training would reduce the inflammatory profile of mRNA in both locations, in particular because the data were generated from pigs with CAD. Intuitively, exercise might have been anticipated to reduce atherosclerosis risk by dampening inflammation in cEAT especially because of its closer proximity to the coronary arteries than mEAT. This was not the case, putatively because of associated "inside-to outside" inflammatory signaling $(35,44)$ emanating from atherogenesis in underling intima-medial coronary plaques, which were demonstrated histologically in our experimental animals. Our designation of cEAT as epicardial adipose tissue directly on or around coronary artery, and mEAT as epicardial adipose tissue on the myocardium but not near or touching an artery was arbitrary but in keeping with radiological definitions of the two entities $(27,30)$. Our findings support a recent report of a difference between cEAT compared with mEAT from adult human donor hearts without macroscopic evidence of CAD in that cEAT was more proinflammatory, releasing greater amounts of monocyte chemoattractant-1 in vitro (8). Despite the significance of the differences between the SED and EX pigs, we acknowledge that a larger sample size might have potentially yielded more significant differences in comparing cEAT as opposed to mEAT expression data. However, if we assume the differences are real without regard to standard errors or statistical tests, the data are as follows: PAI-1 was $-59 \%$ in mEAT and $-14 \%$ in cEAT, eNOS was, respectively, $-56 \%$ and $-2 \%$, cyclophilin was $-41 \%$ and $-3 \%$, PGDS was $-45 \%$ and $-19 \%$, IL-1Ra was $-64 \%$ and $+23 \%$, IL- 6 was $-65 \%$ and $-6 \%$, cytochrome $c$ oxidase was $-30 \%$ and $+40 \%$, IL- 8 was $-65 \%$ and $+20 \%$, UCP-2 was $-50 \%$ and $-22 \%$, while superoxide dismutase was $-48 \%$ and $-35 \%$ and both were significant. It was only with regard to UCP2 and PGDS that there might have been an effect in cEAT that might have been significant if we had a larger number of exercise-trained pigs, but in any case, the effect on UCP-2 and PGDS was, at most, half of that seen in mEAT.

The two EAT components seem to differ metabolically. In the SED pigs, mEAT had more cytochrome $c$ oxidase, indicating more mitochondrial oxidizing units per unit of adipose tissue than EX pigs. However, there was significantly more mEAT mass in EX pigs, so that the total amount of cytochrome $c$ oxidase may not decrease in EX pigs. Likewise, even though lipolytic perilipin and FABP4 expression was the same in cEAT and mEAT before and after exercise, it is possible that the higher mEAT mass postexercise reflects more total mEAT lipolysis that could possibly deliver free fatty acids locally to the hypertrophied myocardium. Superoxide dismutase decreased with aerobic exercise training in both cEAT and mEAT. The enzyme catalyzes the conversion of superoxide to $\mathrm{H}_{2} \mathrm{O}_{2}$ and oxygen (46). Exercise training enhances antioxidant capacity via reactive oxygen species scavenging (46) and has been shown to increase in rat white visceral adipose tissue with exercise (45). The disparate results may relate to the source of tissue analysis, the experimental animal model, and the presence of underlying CAD. In SED pigs, apart from cytochrome $c$ oxidase, mEAT and cEAT showed few differences but differed from VAT and SAT. Compared with SAT, there was far less heme oxygenase, angiotensinogen, adiponectin, perilipin, and FABP4 in both cEAT and mEAT. It is unclear what 

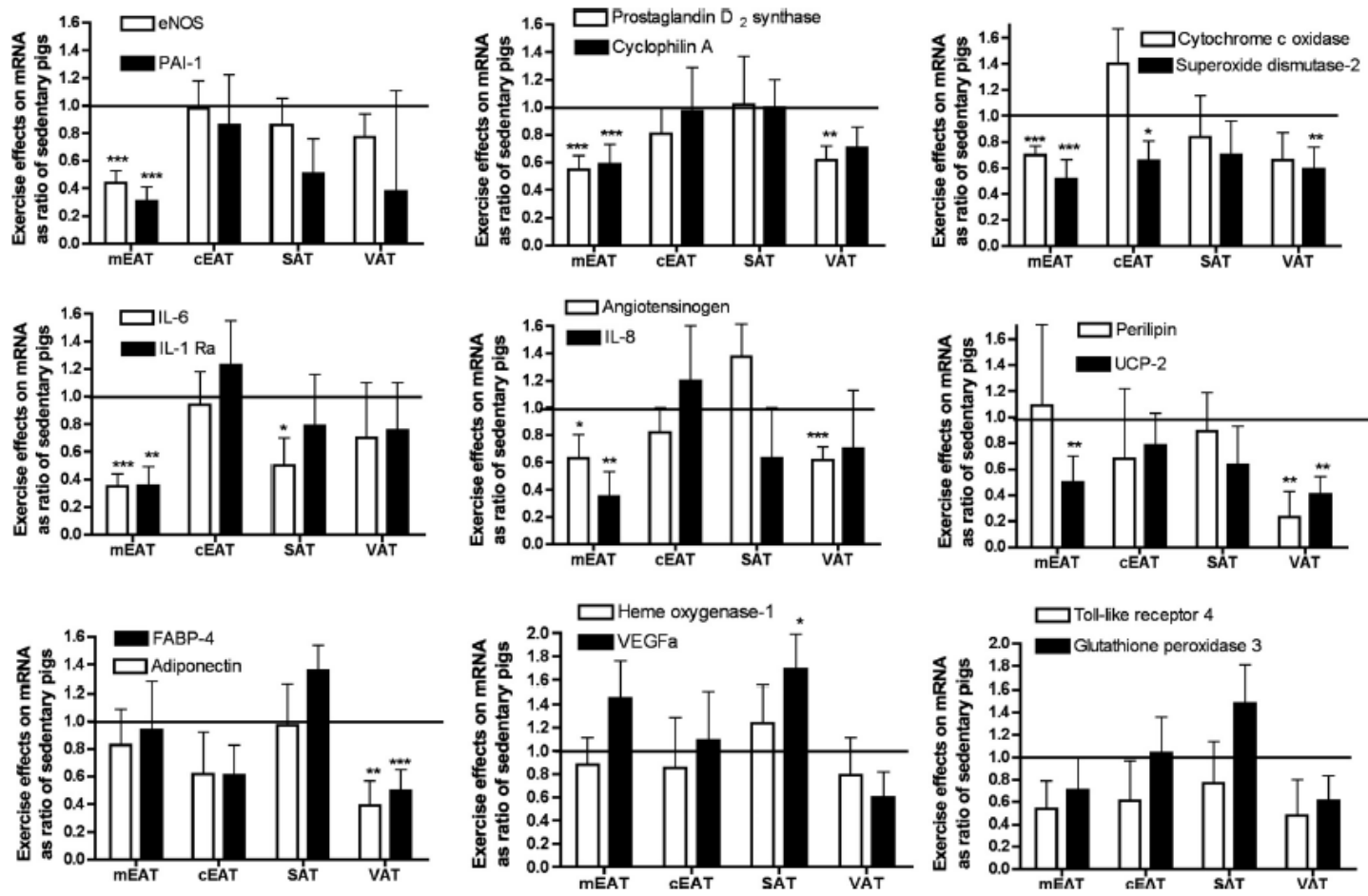

Fig. 6. Comparison of peri-coronary EAT (cEAT), peri-myocardial EAT (mEAT), VAT, and SAT gene expression in exercised pigs to that in sedentary pigs. The mRNA values are the ratios \pm SE of the mRNA expression for adipose tissue from five exercised (EX) pigs compared with that in 8 sedentary (SED). Ratios greater than 1.0 indicate that the level of expression was enhanced by exercise, while ratios less than 1.0 indicate that the level of expression was reduced by aerobic exercise. The horizontal line at 1.0 indicates a ratio (SED/EX) where the expression of mRNAs were equal for SED and EX (i.e., the means were identical). A bar below this horizontal line indicates that EX lowered the expression of the given mRNA. Statistically significant differences between expression in EX mRNA compared with SED mRNA are shown as follows: $* P<0.05,{ }^{* *} P<0.025,{ }^{* * *} P<0.005$

this reflects, but this suggests a different function for EAT compared with omental VAT. In addition, with the exception of PGDS, none of the putative inflammatory markers such as IL-8, IL-1Ra, angiotensinogen, TLR4, visfatin, PAI-1, or IL-6 were elevated in EAT.

Aerobic exercise training did not significantly alter cEAT mass but increased mEAT mass. Pigs lack functional UCP-1 (2), which, when coupled with a decrease in UCP-2 in EX pigs' mEAT, in part may account for the significant increase in EAT mass in EX pigs. On the other hand, perilipin, a regulator of lipolysis, was not downregulated in VAT as it was in EAT. It is possible that the expansion of the myocardium that occurred during exercise also expands adipose tissue at the periphery of the organ and this is less inflamed because it is further away from the toxic influence of inflamed endothelium in the sedentary atherosclerotic heart, which has a poorer oxygen supply. If this is the case then it is curious that the greatest effects of exercise are not seen in the fat closest to the putative inflammatory source. It may be that adipose tissue in the growth phase of expansion is more insulin responsive and exhibits less inflammation. Further studies are needed to substantiate whether cEAT and mEAT have distinct functions. Hypothetically, cEAT may alter vasomotion like peri-vascular fat around arteries in other extracardiac sites $(15,16)$.
It has been previously suggested that, due to its immediate anatomic apposition to the heart without fascial boundaries, EAT might secrete a number of inflammatory cytokines that interact with and modulate the coronary arteries and myocardium via paracrine and vasocrine pathways (25). We found that IL-1 $\beta$, IL-6, IL-8, PAI-1, and adiponectin were expressed in both cEAT and mEAT in our sedentary pig model, suggesting that similar paracrine interactions might exist in the pig heart, but whether these inflammatory genes were altered by underlying $\mathrm{CAD}$ is unknown due to the absence of a control group of similar strain pigs with normal coronaries.

Omental and epicardial adipose tissue exhibited different responses to aerobic exercise training. Like VAT, EAT is, by definition, a visceral adipose tissue possessing several common characteristics with mesenteric/omental VAT: EAT and VAT share an embryologic origin in the splanchnopleuric mesoderm affiliated with the gut $(6,24,44)$, EAT and VAT increase in obesity (27) and decrease with weight loss (30), and both EAT and VAT are associated with cardiovascular risk factors (6, 10). Given the similarities between EAT and VAT, a similar exercise response could have been expected. However, in this pig model, exercise did not downregulate inflammatory and redox genes in VAT except for PGDS and MnSOD, respectively, but it did lower expression of adipocyte-specific adi- 
ponectin, FABP4, and perilipin mRNAs, which was not observed in cEAT and mEAT. Adiponectin is an anti-inflammatory adipokine that inhibits TNF- $\alpha$ production and stimulates production of other anti-inflammatory cytokines (57). Adiponectin blood levels are reduced in obesity (3) and reduced in hypoxia (56). Increased physical activity is associated with increased levels of adiponectin $(43,57)$. The interpretation of the decrease in adiponectin in VAT but not in SAT is limited by the absence of any direct quantitation of VAT mass and blood adiponectin levels in our experiments especially since exercise training in humans has been shown to reduce VAT in the absence of significant weight loss $(48,49)$.

Most subcutaneous adipose tissue mRNAs did not respond to aerobic exercise training. With the exception of VEGFa mRNA (up) and IL-6 mRNA (down), SAT showed no response to aerobic exercise training in 11 of the mRNAs that were affected by aerobic exercise training in VAT and/or EAT. Unlike VAT and EAT, SAT is derived from a different mesodermal embryologic origin, is not associated with increased metabolic risk, and may be protective against metabolic disorders (51). Both VEGFa (18) and IL-6 (12) have proinflammatory characteristics. IL-6 is currently considered one of the primary mediators of chronic inflammation, and adipose tissue accounts for $15-35 \%$ of circulating levels (55). VEGF is proangiogenic (23) and plasma levels have been shown to increase with exercise training (32). One possible explanation for the opposite response seen in VEGFa and IL-6 is that the increase in VEGFa mRNA in EX pigs' SAT increased blood flow, which flow may result in better oxygenation and less inflammation, and thus less IL-6 mRNA.

In conclusion, this is the first study to investigate the response of mRNA levels to aerobic exercise training in four adipose tissue depots of pigs with FH and CAD. The major finding is that aerobic exercise training reduced the inflammatory response in mEAT but not cEAT. This suggests that epicardial adipose tissue is not homogeneous in its responses to aerobic exercise training. After exercise, VAT showed decreased preferential expression of adiponectin and other adipocyte-specific genes. None of the 19 genes in SAT responded to aerobic exercise training, except IL-6, which went down, and VEGFa mRNA, which went up. This indicates that responses of other inflammatory mRNAs in subcutaneous adipose tissue cannot be extrapolated to omental and heart adipose tissues in exercise intervention studies.

\section{ACKNOWLEDGMENTS}

We thank Pam Thorne, Dave Harah, and Jennifer Casati for their technical assistance.

\section{GRANTS}

This research was supported by National Institutes of Health Grants HL-52490 and T32-AR-048523 and The Cardiometabolic Disease Research Foundation, Memphis, TN.

\section{DISCLOSURES}

No conflicts of interest, financial or otherwise, are declared by the author(s).

\section{REFERENCES}

1. Berg $\mathbf{A H}$, Scherer PE. Adipose tissue, inflammation, and cardiovascular disease. Circ Res 96: 939-949, 2005.
2. Berg F, Gustafson U, Andersson L. The uncoupling protein 1 gene (UCP1) is disrupted in the pig lineage: a genetic explanation for poor thermoregulation in piglets. PLoS Genet 8: 1178-1181, 2006.

3. Berk ES, Kovera AJ, Boozer CN, Pi-Sunyer FX, Johnson JA, Albu JB. Adiponectin levels during low- and high-fat eucaloric diets in lean and obese women. Obes Res 13:1566-1571, 2005.

4. Bergman RN, Kim SP, Catalano KJ, Hsu IR, Chiu JD, Kabir M, Hucking K, Ader M. Why visceral fat is bad: mechanisms of the metabolic syndrome. Obesity 14: 16S-19S, 2006.

5. Casiglia E, Palatini P, Da Ros S, Pagliara V, Puato M, Dorigatti F, Pauletto P. Effect of blood pressure and physical activity on carotid artery intima-media thickness in stage 1 hypertensives and controls. $\mathrm{Am} \mathrm{J}$ Hypertens 13: 1256-1262, 2000.

6. Chaowalit N, Lopez-Jimenez F. Epicardial adipose tissue: friendly companion or hazardous neighbor for adjacent coronary arteries? Eur Heart $J$ 29: 695-697, 2008.

7. Chaston TB, Dixon JB. Factors associated with percent change in visceral versus subcutaneous abdominal fat during weight loss: findings from a systematic review. Int J Obes (Lond) 32: 619-628, 2008

8. Chatterjee TK, Stoll LL, Denning GM, Harrelson A, Blomkalns AL, Idelman G, Rothenberg FG, Neltner B, Romig-Martin SA, Dickson EW, Rudich S, Weintraub NL. Proinflammatory phenotype of perivascular adipocytes: influence of high-fat feeding. Circ Res 104: 541-549, 2009.

9. Corradi D, Maestri R, Callegari S, Pastori P, Goldoni M, Luong TV, Bordi C. The ventricular epicardial fat is related to the myocardial mass in normal, ischemic and hypertrophic hearts. Cardiovasc Pathol 13: 313-316, 2004

10. Dagenias GR, Yi Q, Mann JF, Bosch J, Pogue J, Yusif S. Prognostic impact of body weight and abdominal obesity in women and men with cardiovascular disease. Am Heart J 149: 54-60, 2005.

11. Dyson MC, Alloosh M, Vuchetich JP, Mokelke EA, Sturek M. Components of metabolic syndrome and coronary artery disease in female Ossabaw swine fed excess atherogenic diet. Comp Med 56: 35-45, 2006.

12. Eder K, Baffy N, Falus A, Fulop AK. The major inflammatory mediator interleukin-6 and obesity. Inflamm Res 58: 727-736, 2009.

13. Fain JN, Buehrer B, Bahouth SW, Tichansky DS, Madan AK. Comparison of messenger RNA distribution for 60 proteins in fat cells vs. the nonfat cells of human omental adipose tissue. Metabolism 57: 1005-1015, 2008.

14. Fain JN, Sacks HS, Buehrer B, Bahouth SW, Garrett Wolf RY E, Carter RA, Tichansky DS, Madan AK. Identification of omentin mRNA in human epicardial adipose tissue: comparison to omentin in subcutaneous, internal mammary artery periadventitial and visceral abdominal depots. Intl J Obes 32: 810-815, 2008.

15. Fantuzzi G, Mazzone F. Adipose tissue and atherosclerosis. Exploring the connection. Arterioscler Thromb Vasc Biol 27: 996-1003, 2007.

16. Gao YJ, Takemori K, Su LY, An WS, Lu C, Sharma AM, Lee RM. Perivascular adipose tissue promotes vasoconstriction: the role of superoxide anion. Cardiovasc Res 71: 363-373, 2006.

17. Guzik TJ, Marvar PJ, Czesnikiewicz-Guzik M, Korbut R. Perivascular adipose tissue as a messenger of the brain-vessel axis: role in vascular inflammation and dysfunction. J Physiol Pharmacol 58: 591-610, 2007.

18. Harvey HL. The link between lymphatic function and adipose biology. Ann NY Acad Sci 1131: 82-88, 2008.

19. Hasler-Rapacz J, Ellegren H, Fridolfsson AK, Kirkpatrick B, Kirk S, Andersson L, Rapacz J. Identification of a mutation in the low density lipoprotein receptor gene associated with recessive familial hypercholesterolemia in swine. Am J Med Genet 76: 379-386, 1998.

20. Hasler-Rapacz J, Ellegren H, Fridolfsson AK, Kirkpatrick B, Kirk S, Andersson L, Rapacz J. Identification of a mutation in the low density lipoprotein receptor gene associated with recessive familial hypercholesterolemia in swine. Am J Med Genet 76: 379-386, 1998.

21. Hasler-Rapacz J, Kempen H,J, Princen HM, Kudchodkar BJ, Lacko A, Rapacz J. Effects of simvastatin on plasma lipids and apolipoproteins in familial hypercholesterolemic swine. Arterioscler Thromb Vasc Biol 16: 137-143, 1996.

22. Hasler-Rapacz J, Prescott MF, Von Linden-Reed J, Rapacz JM Jr, Hu Z, Rapacz J.Elevated concentrations of plasma lipids and apolipoproteins B, C-III, and E are associated with the progression of coronary artery disease in familial hypercholesterolemic swine. Arterioscler Thromb 15: 583-592, 1995.

23. Hausman GJ, Richardson RL. Adipose tissue angiogenesis. J Anim Sci 82: 925-934, 2004. 
24. Ho E, Shimada Y. Formation of the epicardium studied with the scanning electron microscope. Dev Biol 66: 579-85, 1978.

25. Iacobellis G, Gao YJ, Sharma AM. Do cardiac and perivascular adipose tissue play a role in atherosclerosis? Curr Diab Rep 8: 20-24, 2008.

26. Iacobellis G, Corradi D, Sharma AM. Epicardial adipose tissue: anatomic, biomolecular and clinical relationship with the heart. Nat Clin Pract Cardiovasc Med 2: 536-543, 2005.

27. Iacobellis G, Ribaundo MC, Assael F, Vecci E, Tiberti C, Zappaterreno A, Di Mario U, Leonetti F. Echocardiographic epicardial adipose tissue is related to anthropometric and clinical parameters of metabolic syndrome: a new indicator of cardiovascular risk. J Clin Endocrinol Metab 88: 5165-5168, 2003

28. Junyent M, Cofan M, Nunez I, Gilabert R, Zambon D, Ros E. Influence of HDL cholesterol on preclinical carotid atherosclerosis in familial hypercholesterolemia. Atherioscler Thromb Vasc Biol 26:10071013.

29. Kim MK, Tanaka K, Kim MJ, Matuso T, Endo T, Tomita T, Maeda S, Ajisaka R. Comparison of epicardial, abdominal and regional fat compartments in response to weight loss. Nutr Metab Cardiovasc Dis 19: $760-766,2009$

30. Kim MK, Tomita T, Kim MJ, Sasai H, Maeda S, Tanaka K. Aerobic exercise training reduces epicardial fat in obese men. $J$ Appl Physiol 106: 5-11, 2009.

31. Langheim S, Dreas L, Veschini L, Maisano F, Foglieni C, Ferrarello S, Sinagra G, Zingone B, Alfieri O, Ferrero E, Maseri A, Ruotolo G. Increased expression and secretion of resistin in epicardial adipose tissue of patients with acute coronary syndrome. Am J Physiol Heart Circ Physiol 298: H746-H753, 2010.

32. Laufs U, Werner N, Link A, Endres M, Wassmann S, Jurgens K, Miche E, Bohm M, Nickening G. Physical training increases endothelial progenitor cells, inhibits neointima formation, and enhances angiogenesis. Circulation 109: 220-226, 2004.

33. Lee DM, Mok T, Hasler-Rapacz J, Rapacz J. Concentrations and compositions of plasma lipoprotein subfractions of Lpb5-Lpul homozygous and heterozygous swine with hypercholesterolemia. J Lipid Res 31: 839-847, 1990.

34. Mayet J, Stanton AV, Chapman N, Foale RA, Hughes AD, Thom SA. Is carotid artery intima-media thickening a reliable marker of early atherosclerosis? J Cardiovasc Risk 9: 77-81, 2002.

35. Mazurek T, Zhang L, Zalewski A, Mannion JD, Diehl JT, Arafat H, Sarov-Blat L, O'Brien S, Keiper EA, Hohnson AG, Martin J, Goldstein BJ, Shi Y. Human epicardial adipose tissue is a source of inflammatory mediators. Circulation 108: 2460-2466, 2003.

36. Nelson RH, Prasad A, Lerman A, Miles JM. Myocardial uptake of circulating triglycerides in non-diabetic patients with heart disease. Diabetes 56: 527-530, 2007.

37. Physical Activity Guidelines Advisory Committee. Physical Activity Guidelines Advisory Committee Report, 2008. Washington, DC: U.S. Department of Health and Human Services, 2008.

38. Prescott MF, Hasler-Rapacz J, Von Linden-Reed J, Rapacz J. Familial hypercholesterolemia associated with coronary atherosclerosis in swine bearing different alleles for apolipoprotein B. Ann NY Acad Sci 748: 283-293, 1995.

39. Prescott MF, McBride CH, Hasler-Rapacz J, Von Linden J, Rapacz J. Development of complex atherosclerotic lesions in pigs with inherited
hyper-LDL cholesterolemia bearing mutant alleles for apolipoprotein B. Am J Pathol 139: 139-147, 1991.

40. Rapacz J, Hasler-Rapacz JO, Hu ZL, Rapacz JM, Vogeli P, Hojny J, Janik A. Identification of new apolipoprotein B epitopes and haplotypes and their distribution in swine populations. Anim Gent 25: 51s-57s, 1994.

41. Rapacz J, Hasler-Rapacz J, Taylor KM, Checovich WJ, Attie AD. Lipoprotein mutations in pigs are associated with elevated plasma cholesterol and atherosclerosis. Science 234: 1573-1577, 1986.

42. Reifenberger MS, Turk JR, Newcomer SC, Booth FW, Laughlin MH. Perivascular fat alters reactivity of coronary artery: effects of diet and exercise. Med Sci Sports Exerc 39: 2125-2134, 2007.

43. Ring-Dimitriou S, Paulweber B, vonDuvillard SP, Stadlmann M, LeMura LM, Lang J, Muller E. The effect of physical activity and physical fitness on plasma adiponectin in adults with predisposition to metabolic syndrome. Eur J Appl Physiol 98: 472-481, 2006

44. Sacks HS, Fain JN. Human epicardial adipose tissue: a review. Am Heart J 153: 907-917, 2007.

45. Sakurai T, Izawa T, Ogasawara JE, Shirato K, Imaizumi K, Takahashi $\mathbf{K}$, Ishida $\mathbf{H}$, Ohno $\mathbf{H}$. Exercise training decreases expression of inflammation-related adipokines through reduction of oxidative stress in rat white adipose tissue. Biochem Biophys Res Commun 379: 605-609, 2009

46. Sen CK. Oxidants and antioxidants in exercise. J Appl Physiol 79: 675-686, 1995.

47. Stern N, Marcus Y. Perivascular fat: innocent bystander or active player in vascular disease? J Cardiometab Synd 1: 115-120, 2006.

48. Thomas DE, Elliott EJ, Naughton GA. Exercise for type 2 diabetes mellitus. Cochrane Database Syst Rev 3: CD002968, 2006 (doi:10.1002 14651858.CD002968.pub2).

49. Thomas EL, Brynes AE, McCarthy J, Goldstone AP, Hajnal JV, Saeed N, Frost G, Bell JD. Preferential loss of visceral fat following aerobic exercise, measured by magnetic resonance imaging. Lipids 35: aerobic exercise,

50. van der Graff A, Kastelein JJP, Wiegman A. Heterozygous familial hypercholesterolaemia in childhood: cardiovascular risk prevention. $J$ Inherit Metab Dis 32: 699-705, 2009.

51. Virtue S, Vidal-Puig A. It's not how fat you are, it's what you do with it that counts. PLoS Biol 23: 1819-1823, 2008.

52. Wilson PWF, D'Agostino RB, Levy D, Belanger AM, Silbershatz H, Kannel WB. Prediction of coronary heart disease using risk factor categories. Circulation 97: 1837-1847, 1998.

53. Woodman CR, Ingram D, Bonagura J, Laughlin MH. Exercise training improves femoral artery blood flow responses to endothelium-dependent dilators in hypercholesterolemic pigs. Am J Physiol Heart Circ Physiol 290: H2362-H2368, 2006.

54. Woodman CR, Turk JR, Willams DP, Laughlin MH. Exercise training preserves endothelium-dependent relaxation in brachial arteries from hyperlipidemic pigs. J Appl Physiol 94: 2017-2026, 2003.

55. Yang Y, Ju D, Zhang M, Yang G. Interleukin-6 stimulates lipolysis in porcine adipocytes. Endocrine 22: 261-269, 2008.

56. Ye J, Gao Z, Yin J, He Q. Hypoxia is a potential risk factor for chronic inflammation and adiponectin reduction in adipose tissue of ob/ob/ and dietary obese mice. Am J Physiol Endocrinol Metab 293: E1118-E1128, 2007

57. You T, Nicklas BJ. Effects of exercise on adipokines and the metabolic syndrome. Curr Diab Rep 8: 7-11, 2008. 


\section{APPENDIX C}

11ßHSD-1 enzyme activity assay, immunohistochemistry staining, and lipolysis in FHM pigs

Unpublished data 
The overall question for the $11 \beta \mathrm{HSD}-1 \mathrm{FHM}$ pigs was: Does $11 \beta \mathrm{HSD}-1$ activity in various adipose tissue depots correlate with lipolysis?

For my initial experiment using FHM4 pigs, I hypothesized that exercise would decrease omental adipose tissue by maintaining smaller adipocytes and increasing lipolysis. I examined three variables: 1) adipose tissue distribution, 2) adipocyte size, and 3) lipolysis. I found that exercise did not reduce omental adipose tissue in the exercised pigs (Table C1) and omental adipocytes were not smaller (Figure C2), but omental adipose tissue had higher basal lipolysis than subcutaneous adipose tissue, and exercise seems to augment this response (Figure C3). Also, we found that exercise may increase $11 \beta \mathrm{HSD}-1$ protein and activity in FHM5 pig adipose tissue (epicardial, omental, and subcutaneous adipose tissue) (Arce and Ganjam, unpublished data) (Figure C4 \& C6). In addition, we found that omental adipose tissue has higher levels of $11 \beta \mathrm{HSD}-1$ protein and activity than subcutaneous adipose tissue (Arce and Ganjam, unpublished data). Typically, $11 \beta \mathrm{HSD}-1$ activates glucocorticoids, but, as of now, we are not certain if $11 \beta \mathrm{HSD}-1$ in exercised adipose tissue activates (reduces) or inactivates (oxidizes) glucocorticoids. The connection between $11 \beta \mathrm{HSD}-1$ and lipolysis is that cortisol (active form of glucocorticoids) stimulates lipolysis.

In addition to the above experiments (fat distribution, adipocyte size, and lipolysis), another thing I would like to do is measure both the reductase and 
oxidase activity of $11 \beta \mathrm{HSD}-1$ to determine which direction the enzyme is going in our pig adipose samples (subcutaneous, omental, and epicardial adipose tissue).

Given that $11 \beta \mathrm{HSD}-1$ is predominantly an activator (reductase) of glucocorticoids, and since glucocorticoids are known to increase lipolysis, for the FHM6 pigs, I hypothesize that exercised pigs will exhibit more $11 \beta \mathrm{HSD}-1$ protein and reductase activity in omental adipose tissue, which will promote increased omental lipolysis and possibly contribute to adipose tissue redistribution away from omental adipose tissue.

Since there are only three other publications investigating $11 \beta \mathrm{HSD}-1$ and exercise, this data would be novel and contribute to the growing body of $11 \beta \mathrm{HSD}-1$ literature and help us understand how this enzyme affects adipose tissue in an exercise model. In addition, there is no data on epicardial adipose tissue in this context; this would be the first time exercise, lipolysis, and $11 \beta \mathrm{HSD}$ 1 would be investigated in epicardial adipose tissue.

Below are figures from data that has been collected for this project. These figures would be similar to the type of figures that I feel would be necessary to include in a manuscript to tell this story. Additional information I would like to collect is indicated in red italics under the figures. 


\section{FHM4}

Sed (6), EX (8)

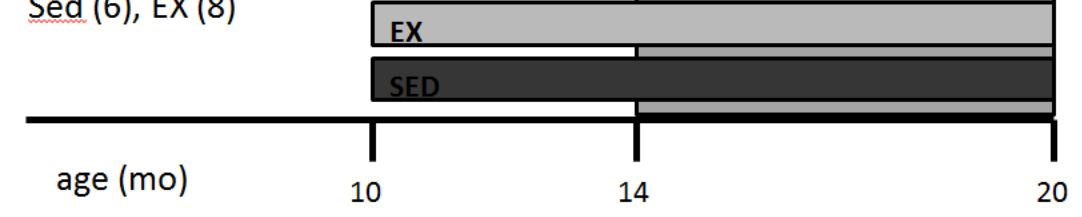

Figure C1. Experimental design

Two cohorts of pigs were randomly selected to be either exercise trained (EX) on a treadmill or remain sedentary (SED) (see Appendix B for exercise protocol). At 14 months of age, both groups were put on a high fat diet. Pigs were sacrificed at 20 months of age.

Table C1. Pig Characteristics

\begin{tabular}{|l|c|cc|}
\hline & FHM 4 & FHM4 EX & FHM4 SED \\
& $\mathbf{n = 1 4}$ & $\mathbf{n = 8}$ & $\mathbf{n = 6}$ \\
\hline Body Weight (kg) & $85.3 \pm 2.3$ & $86.1 \pm 2.6$ & $84.2 \pm 4.3$ \\
\%BF (DXA) & $34.7 \pm 1.2$ & $35.7 \pm 1.1$ & $33.4 \pm 2.6$ \\
Fat wt (kg) & $29.7 \pm 1.5$ & $30.8 \pm 1.7$ & $28.3 \pm 2.9$ \\
OM weight (g) & $256.4 \pm 28.1$ & $299.8 \pm 40.4$ & $198.5 \pm 23.7$ \\
\%OM of total fat & $0.84 \pm 0.06$ & $0.95 \pm 0.09$ & $0.70 \pm 0.05$ \\
Waist Circ. (in) & $42.1 \pm 0.7$ & $42.2 \pm 0.65$ & $42.0 \pm 1.4$ \\
WC/BW & $0.50 \pm 0.01$ & $0.49 \pm 0.01$ & $0.50 \pm 0.01$ \\
\hline
\end{tabular}

There was a trend for more omental weight $(p=0.072)$ and percent of total omental fat $(0.054)$ in the exercised pigs. 
Figure C2. Adipocyte size from FHM4 pigs.

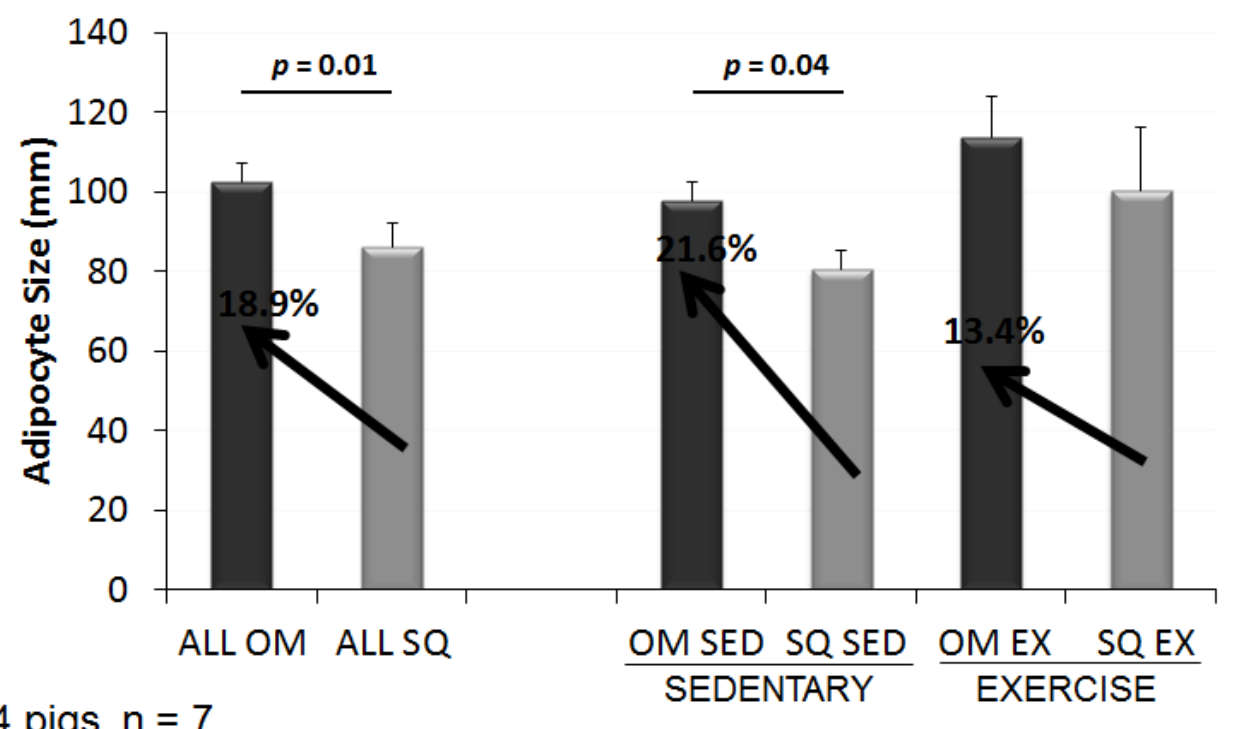

FHM 4 pigs, $n=7$

SED: $\mathrm{n}=5$

EX: $n=2$

Omental adipocytes are larger than subcutaneous adipocytes. Exercise may mitigate this effect.

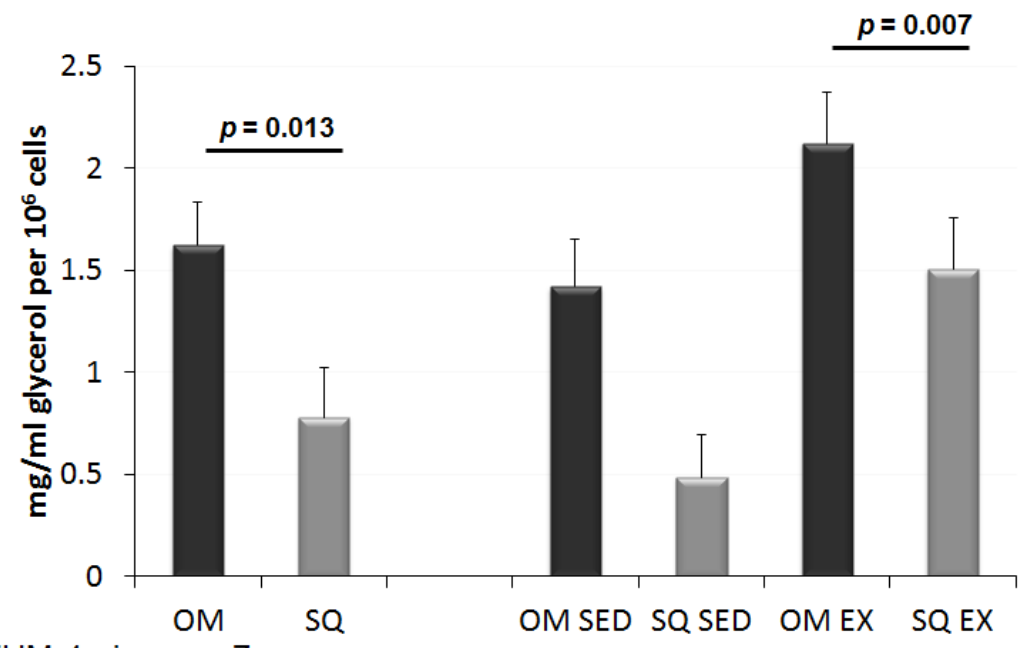

FHM 4 pigs, $n=7$

SED: $n=5$

EX: $n=2$

Figure C3. Lipolysis from FHM4 pigs. 
A glycerol assay was used to evaluate lipolysis in these pigs. Omental adipocytes show more basal metabolic activity than subcutaneous.

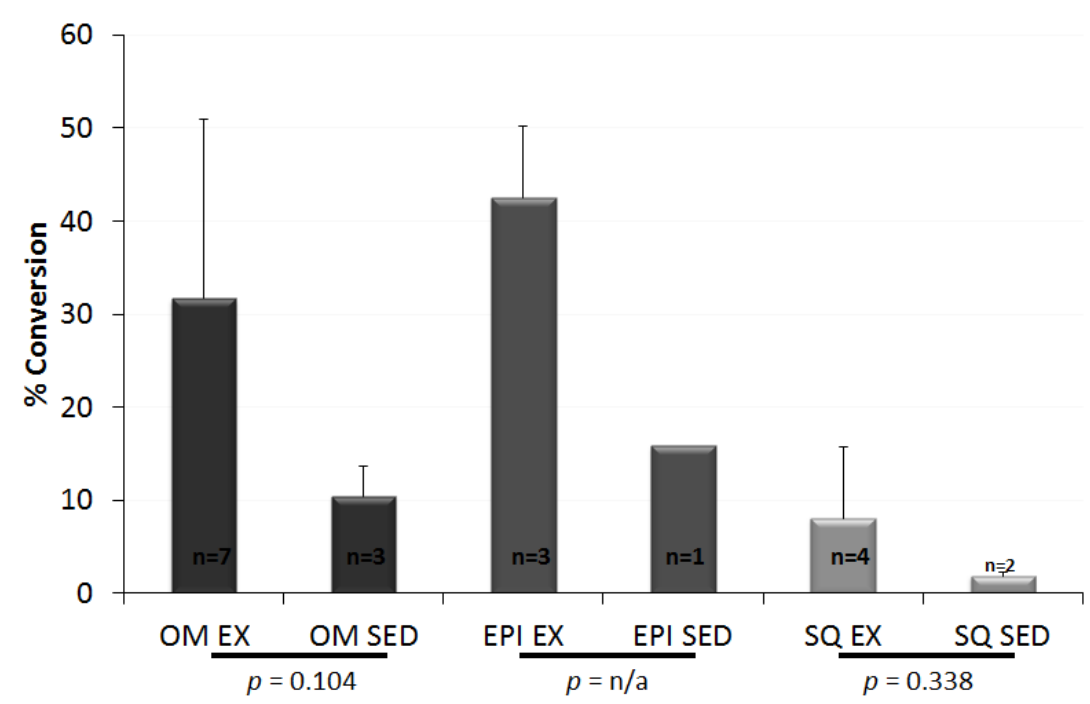

Figure C4. 11ßHSD-1 enzyme activity in FHM5 pigs (Oxidase activity)

There appeared to be more $11 \beta \mathrm{HSD}-1$ oxidase activity in exercise pigs. This data was confirmed with immunohistochemistry staining for $11 \beta \mathrm{HSD}$ by $\mathrm{Dr}$. Arturo Arce. The next two figures are work done by Dr. Arce.

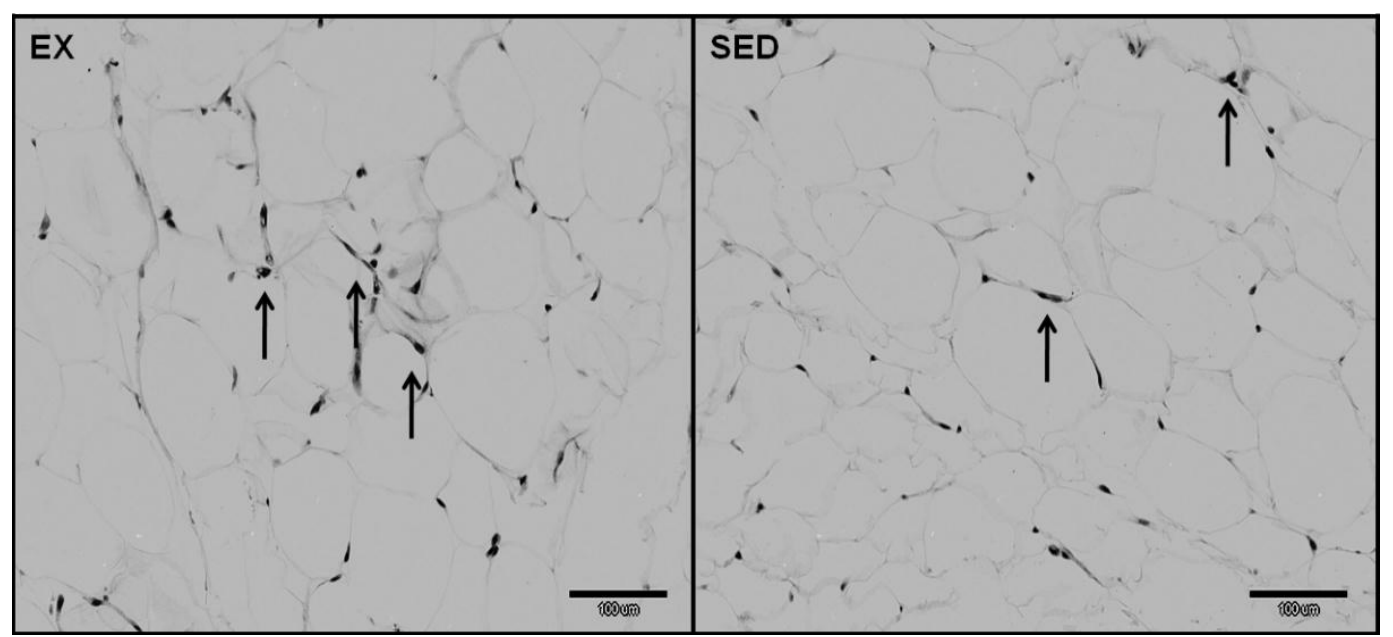

Figure C5. IHC staining for 11ßHSD-1 in FHM5 adipose tissue (from Arce)

EX pigs had more $11 \beta \mathrm{HSD}-1$ staining than SED pigs. The data is quantified below. 


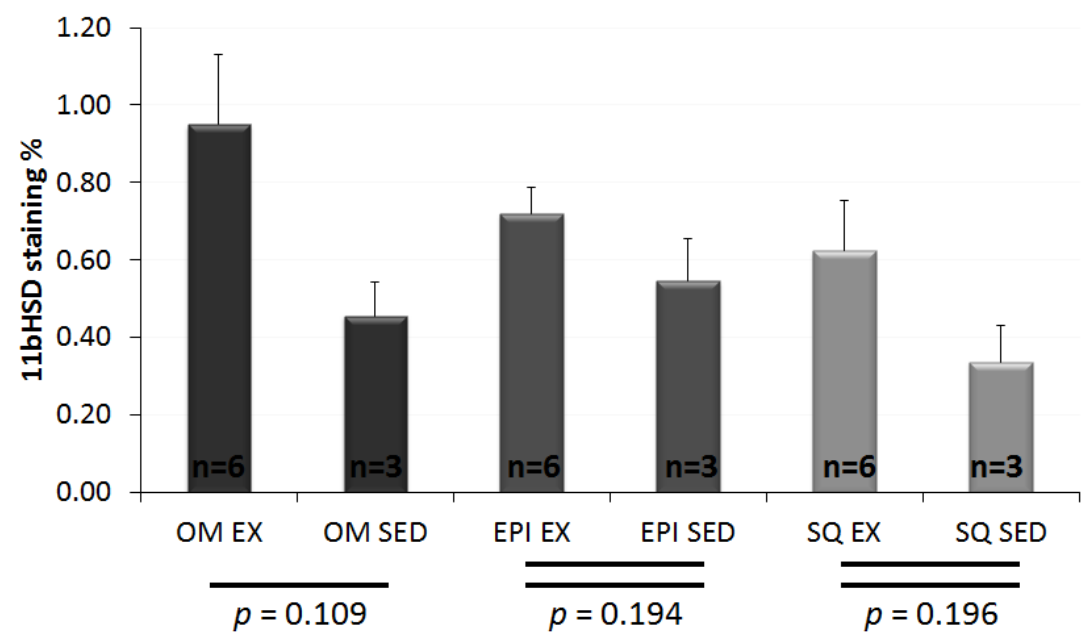

Figure C6. 11ßHSD-1 protein in FHM4 pigs (from Arce)

EX pigs appeared to have more $11 \beta \mathrm{HSD}-1$ protein than SED pigs.

Unfortunately, the assay was unable to be performed on any subsequent cohorts of pigs, and the project was halted. 


\title{
APPENDIX D
}

Investigation on chronic high fat diet versus low fat diet on Wistar rats: Adipocyte distribution, adipocyte size and lipolysis

\author{
Unpublished data
}


The purpose of this experiment was to evaluate the effect of a high fat diet on body fat, adipose distribution, and lipolysis.

At $\sim 5$ weeks of age, male Wistar rats were randomly selected to either a normal chow diet (LFD) or high fat diet (HFD). Rats were on this diet for 22 weeks, and were sacrificed at $\sim 27$ weeks of age (191-194 days of age). A group of rats was sacrificed at $103-117$ days of age to serve as a young, control.

Plasma was collected at the time of sacrifice for a glycerol assay. The following measurements were performed: weight, length, DXA \% body fat, omental (OM) depot mass and adipocyte diameter, epididymal (EPI) depot mass and adipocyte diameter, and subcutaneous (SQ) adipocyte diameter. Fat mass, lean mass, \% body fat that is OM and \% body fat that is EPI were calculated.

Results are below.

Table D1. Anthropometric and adipose characteristics of young, HFD, and LFD rats.

\begin{tabular}{|c|c|c|c|c|c|c|c|}
\hline & $\begin{array}{c}\text { age at } \\
\text { sacrifice }\end{array}$ & $\begin{array}{c}\text { days on } \\
\text { HFD }\end{array}$ & $\begin{array}{c}\text { weight } \\
\text { (g) }\end{array}$ & $\begin{array}{l}\text { length } \\
\text { (cm) }\end{array}$ & $\%$ BF & $\begin{array}{c}\text { fat } \\
\text { mass (g) }\end{array}$ & $\begin{array}{c}\text { lean } \\
\text { mass (g) }\end{array}$ \\
\hline YOUNG & $110.1 \pm 7.0$ * \# & $n / a$ & $456.6 \pm 24.7 * \#$ & $25.8 \pm 0.7^{*} \#$ & $16.8 \pm 1.3 * \#$ & $76.9 \pm 7.5 * \#$ & $397.8 \pm 21.7 * \#$ \\
\hline LFD & $191.8 \pm 0.9^{\wedge}$ & $\mathrm{n} / \mathrm{a}$ & $724.7 \pm 40.7^{\wedge}$ & $29.0 \pm 0.5^{\wedge}$ & $28.5 \pm 1.0^{\wedge} \#$ & $207.0 \pm 15.6^{\wedge} \#$ & $517.6 \pm 28.3^{\wedge}$ \\
\hline HFD & $193.6 \pm 1.1^{\wedge}$ & $160.3 \pm 1.2$ & $747.2 \pm 27.0^{\wedge}$ & $28.4 \pm 0.5^{\wedge}$ & $39.5 \pm 3.9^{\wedge} *$ & $298.9 \pm 36.1^{\wedge} *$ & $448.3 \pm 24.1^{\wedge}$ \\
\hline \multicolumn{8}{|l|}{ * vs LFD } \\
\hline \multicolumn{8}{|l|}{ \# vs HFD } \\
\hline \multicolumn{8}{|c|}{ ^ vs YOUNG } \\
\hline
\end{tabular}

\begin{tabular}{|c|c|c|c|c|c|c|c|}
\hline & $\begin{array}{c}\text { OM AT } \\
\text { mass (g) }\end{array}$ & $\begin{array}{c}\% \mathrm{BF} \\
\mathrm{OM}\end{array}$ & $\begin{array}{c}\text { EPI AT } \\
\operatorname{mass}(\mathrm{g})\end{array}$ & $\begin{array}{c}\% \text { BF } \\
\text { EPI }\end{array}$ & $\begin{array}{l}\text { OM A size } \\
\text { (mm) }\end{array}$ & $\begin{array}{c}\text { EPI A size } \\
(\mathrm{mm})\end{array}$ & $\begin{array}{c}\text { SQ A size } \\
\text { (mm) }\end{array}$ \\
\hline YOUNG & $1.2 \pm 0.1 * \#$ & $0.3 \pm 0.0 * \#$ & $8.6 \pm 0.7^{*} \#$ & $1.9 \pm 0.1 * \#$ & $64.1 \pm 3.0 \#$ & $64.1 \pm 3.1 \#$ & $60.0 \pm 3.9 \#$ \\
\hline LFD & $3.4 \pm 0.2^{\wedge} \#$ & $0.5 \pm 0.0^{\wedge} \#$ & $19.0 \pm 0.8^{\wedge} \#$ & $2.6 \pm 0.1^{\wedge}$ & $62.8 \pm 2.1 \#$ & $72.9 \pm 3.8 \#$ & $67.0 \pm 1.8$ \\
\hline HFD & $5.1 \pm 0.6^{\wedge} *$ & $0.7 \pm 0.1^{\wedge} *$ & $24.7 \pm 2.2^{\wedge} *$ & $3.3 \pm 0.2^{\wedge}$ & $106.0 \pm 3.4^{\wedge} *$ & $85.7 \pm 3.3^{\wedge} *$ & $81.0 \pm 4.3^{\wedge}$ \\
\hline
\end{tabular}

A lipolysis assay (glycerol assay) was performed from the plasma (Figure D1). The glycerol assay quantifies free glycerol in plasma and is a marker of 
triglyceride hydrolysis. I used a glycerol assay kit from purchased from Sigma (Sigma F6428, G7793).

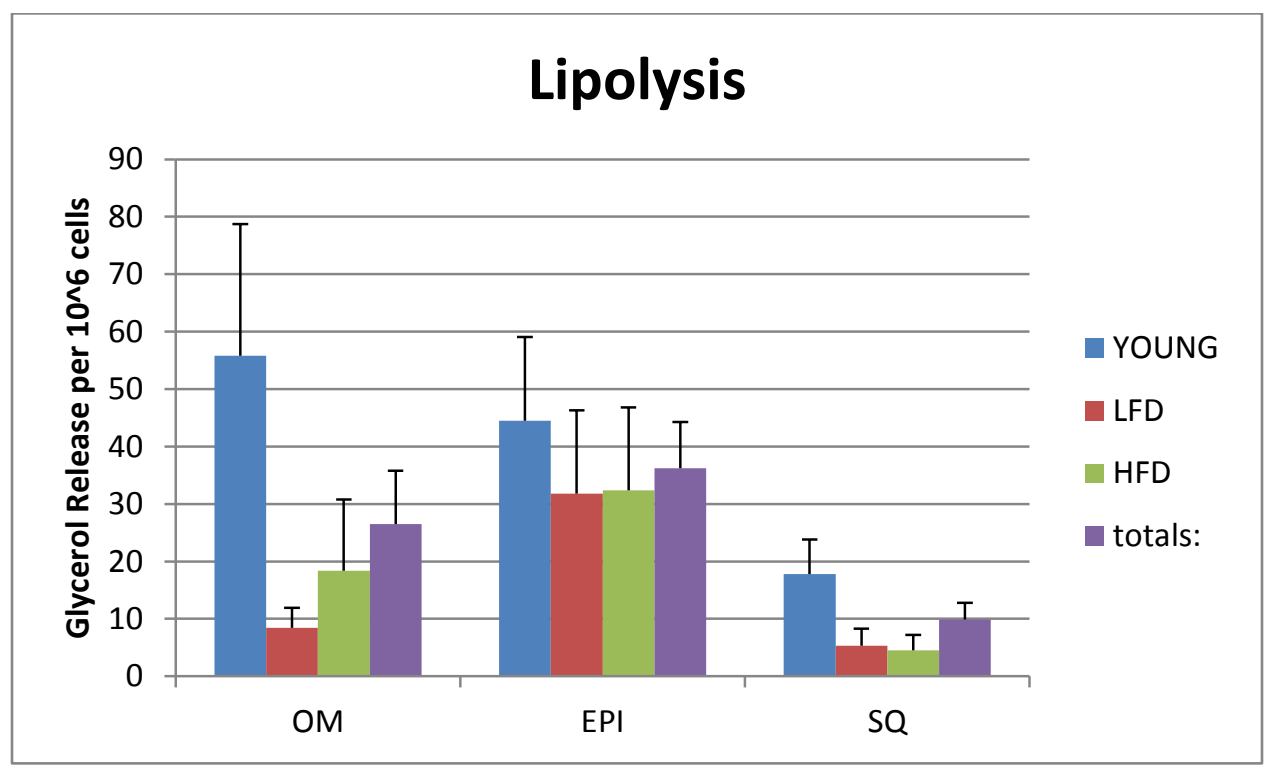

Figure D1. Lipolysis assay on plasma of YOUNG, LFD, and HFD rats. 


\section{VITA}

Joseph Company received his bachelors in Mathematics Education from Goshen College, Goshen IN. He taught high school mathematics for six years before returning to Goshen College for continuing education in Biology, Chemistry, Physiology, and Nutrition. He moved to Columbia, MO in January, 2006 to study Exercise Physiology at the University of Missouri. He received his Master's degree in August, 2006. His thesis compared two body composition measurements, DXA and bioelectric impedance analysis on endurance and strength athletes. His thesis was published in Measurements in Physical Education and Exercise Science in 2010. Joe continued his education in the Biomedical Sciences doctoral program in August, 2008. This work represents his doctoral research, which was completed in May, 2013. 